\title{
Stochastic Modelling of Leading-Edge Noise in Time-Domain using Vortex Particles
}

\author{
Von der Fakultät für Maschinenbau, Elektro- und Energiesysteme \\ der Brandenburgischen Technischen Universität Cottbus-Senftenberg \\ zur Erlangung des akademischen Grades eines \\ Doktor der Ingenieurwissenschaften \\ -Dr.-Ing.-
}

genehmigte Dissertation

vorgelegt von

Sparsh Sharma

geboren am 28.07.1992 in New Delhi

Vorsitzender: Apl. Prof. Dr. rer. nat. habil. Uwe Harlander

Gutachter: Prof. Dr.-Ing. Ennes Sarradj

Gutachter: Prof. Dr.-Ing. Heiko Schmidt

Tag der mündlichen Prüfung: 17.12.2019 
An e-book version of this thesis may be downloaded at https://dx.doi.org/10.26127/BTUOpen-5085 


\section{Zusammenfassung}

Die konzeptionelle und vorläufige Auslegung rotierender Maschinen wie Lüfter, Windturbinen, gegenläufiger offener Rotoren und Hubschrauberblätter erfordert kostengünstige und einfach zu bedienende Werkzeuge, die eine schnelle Geräuschbeurteilung und Optimierungsanalyse ermöglichen. Die neuesten numerischen und experimentellen Methoden sind für die Durchführung einer Optimierungsstudie zu rechenaufwändig, wohingegen kostengünstige Methoden, wie zum Beispiel analytische, bei realistischen Profilgeometrien vor allem im Bereich hoher Frequenzen sowie bei höheren Anstellwinkeln erhebliche Fehler aufweisen können.

Da Turbulenz grundsätzlich eine statistische Größe ist, erscheint es sinnvoll, eine turbulente Strömung sowie auch die Interaktion eines Tragflügel- oder Schaufelprofils mit einer solchen Strömung statistisch zu modellieren. Die Bestimmung der instationären Reaktion des Strömungsprofils ist anschließend für die Vorhersage des entstehenden aerodynamischen Schalls von entscheidender Bedeutung.

Das Hauptziel dieser Arbeit ist die Entwicklung einer neuen kostengünstigen und benutzerfreundlichen numerischen Technik für aeroakustische Konstruktionen, die sich hauptsächlich auf die Wechselwirkung zwischen Tragflügelprofilen und Turbulenzen konzentriert. Die Entwicklung der statistischen Methode gliedert sich in drei Abschnitte; nämlich - 1) der Berechnung der Hintergrundströmung, 2) der Modellierung der statistisch optimierten turbulenten Zuströmung, und 3) der Generierung einer Wirbeldatenbank zur Vorhersage des entstehenden Schalls für mehrere Strömungsfelder, die durch unterschiedliche Werte von Turbulenzintensitäten und Längenskalen gekennzeichnet sind. Im Rahmen dieser Arbeit wird ein neuer Ansatz zur Modellierung einer turbulenten Zuströmung - und damit einer für die Lärmerzeugung wesentlichen Größe - vorgestellt, der eine vergleichsweise schnelle Berechnung erlaubt und nicht auf die Nutzung von Hochleistungscomputern angewiesen ist. Durch diesen Ansatz kann der Einfluss von Turbulenzparametern auf das in Turbomaschinen erzeugte Geräusch quantifiziert werden, wobei auch die geometrischen Parameter des Schaufelblatts bei der Geräuschvorhersage berücksichtigt werden. Die Hintergrundströmung wird bei dieser Methode numerisch simuliert, indem die Wirbeltransportgleichungen in der Lagrange-Form gelöst werden (sogenannte VortexMethoden). Der akustische Einfluss einer endlichen Anzahl von Wirbeln, charakterisiert durch alle möglichen Kombinationen von Wirbelgröße, Zirkulation und Injektionsposition / -zeit, definiert unter Verwendung der Bereiche von Wahrscheinlichkeitsverteilungsfunktionen, die von Injektionspunkten stromaufwärts des Schaufelblatts abgegeben werden, werden vorberechnet und in einer Matrix gespeichert. Diese Methode ist daher im Vergleich zu klassischen VortexMethoden vergleichsweise rechengünstig, da die Auswirkungen von Partikeln vorausberechnet, in einer Matrix gespeichert und schließlich zur Berechnung der Schallentstehung nur noch ausgelesen werden. 



\begin{abstract}
The conceptual designing of rotating machines such as fans, wind turbines, contra-rotating open rotors and helicopter blades require low-cost, easy-to-run tools which allow quick noise assessments and optimisation analyses underlying this phenomenon. The state-of-the-art numerical and experimental methods are far more expensive to conduct an optimisation study, whereas inexpensive methods like the analytical ones can have significant errors in realistic geometries at high-frequency ranges, higher angles of attack. The response to large coherent disturbances and the statistical modelling of turbulence is required because turbulence, by far its nature, is stochastic. Determining the accurate unsteady response of airfoil is crucial for noise prediction. The primary goal of the project is to develop a new low-cost and easyto-use numerical technique for aeroacoustic designs, focussed primarily on airfoil-turbulence interaction. The development of the statistical method is divided into three sections; namely 1) calculating the background flow, 2) modelling of statistically optimised inflow disturbance, 3) constructing a vortex database to predict the noise in multiple flow fields characterised by different values of turbulent intensities and length scales. In the framework of this work a new approach to model inflow turbulence, a significant noise-generating element, is suggested, which does not depend on heavy computations requiring supercomputers. Through this approach, the influence of turbulence parameters on the noise generated in turbomachinery can be quantified. The approach also considers the geometrical parameters of the airfoil in the noise prediction. The background flow is numerically simulated via solving the vorticity transport equations in the Lagrangian form (vortex methods). The acoustic influence of a finite number of vortices, characterised by all the possible combinations of size, circulation and injection position/time defined using the ranges of probability distribution functions, released from injection points upstream of the airfoil are precomputed and stored in a matrix. The method is computationally inexpensive compared to classical vortex methods since the effect due to particles are precomputed, stored in a/an matrix/array. The matrix can be called as a library while predicting the noise from a specific airfoil.
\end{abstract}



To my parents,

Daya and Mahesh Sharma,

to my sister,

Akanksha! 



\section{Acknowledgements}

First and foremost, I would like to express my sincere gratitude to my supervisor Prof. Dr.-Ing. Ennes Sarradj for his constant support and invaluable ideas since the beginning of my doctoral research. Not only he encouraged me to discover new avenues in fluid mechanics and acoustics, but also helped me in becoming an independent researcher today. Prof. Sarradj, through all the three years, I could count on your expertise, advice, and support in both professional and personal matters.

I was honoured to have Prof. Dr.-Ing. Heiko Schmidt as my co-supervisor. Our hourlong meetings at regular interval during my research in Cottbus always ended with crucial suggestions and fruitful ideas. Prof. Schmidt, I highly value your input and comments on the thesis, as well as your friendly and open nature during our conversations.

When I moved to Germany in 2016, Dr.-Ing. Thomas Geyer was the first person I met at Brandenburg Technical University, Cottbus. His guidance helped me in all the time of research and writing of this thesis, understanding of results. Thomas, I am grateful for your constant feedback, cooperation and of course, friendship! Your enthusiasm and immense knowledge of the subject always inspire me to excel.

I thank Anna-Sophia Henke who I got to know at the Chair of Technical Acoustics in Cottbus. Despite our research topics being different, Anna was always available to discuss my work. Anna, thanks a lot!

I thank all my colleagues from BTU Cottbus and Technical University Berlin, especially Roman and Gert who are with me all along this PhD journey. I want to thank Silke Kaschwich for helping me with all administrative paper-work and issues at the University.

This research project would not have been possible without funding from the Graduate Research School (GRS) BTU Cottbus. Thank you, Prof. Dr. phil. Thomas Raab and Mr. Robert Rode, for your support.

I would like to acknowledge the thousands of individuals who have coded for open-source projects for free. It is due to their efforts that scientific work with powerful tools is possible. Special thanks to my family, particularly my parents, who supported me in all my endeavours. I am grateful for their love, affection, caring and sacrifices for educating me and preparing me for my future. To my sister, who is a constant source of determination and joyfulness at the hardest of times.

Finally, I would like to thank Sahitya for your advice, your patience, and your faith. Because you always understood! 



\section{Table of Contents}

Title Page $\quad$ i

Zusammenfassung

Abstract

List of Figures $\quad$ XV

List of Tables $\quad$ xix

Abbreviations $\quad$ xxi

Symbols $\quad$ xxiii

1 Introduction 1

1.1 Airfoil turbulence interaction noise . . . . . . . . . . . . . . 2

1.1.1 Unsteady aerodynamics . . . . . . . . . . . . . . . . . $\underline{3}$

1.1.2 Sound radiation development $\ldots \ldots \ldots \ldots \ldots \ldots$

1.1 .3 Analytical methods . . . . . . . . . . . . . . . $\underline{6}$

1.1.4 Experimental methods . . . . . . . . . . . . . . . . I 7

1.1.5 Numerical methods . . . . . . . . . . . . . . . . . . . . . 10

1.1.5.1 Euler approach . . . . . . . . . . . . . . . . 10

1.1.5.2 Lagrangian approach . . . . . . . . . . . . . 11

1.1.5.3 Frequency domain and time domain comparison . . . . . . 12

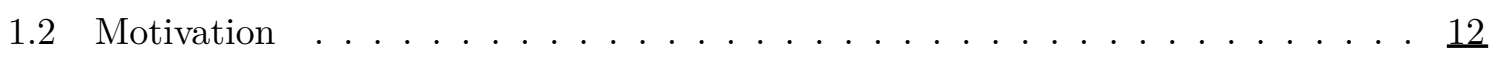

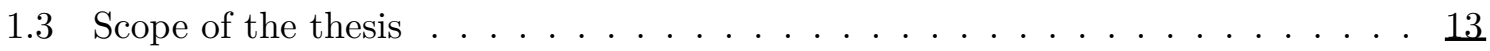

1.4 Thesis structure . . . . . . . . . . . . . . . . . . 14

2 Inviscid and Incompressible Flow $\quad \underline{17}$

2.1 Description of Fluid Behaviour . . . . . . . . . . . . . . . . . 18

2.2 Different Formulations for the Incompressible Navier-Stokes Equation . . . . . 18

2.3 Incompressible Vortical flow . . . . . . . . . . . . . . . . . . . . 19

2.3 .1 Vortex Methods . . . . . . . . . . . . . . . . 21

$2.3 .2 \Psi$-formulation . . . . . . . . . . . . . . . . 21

2.3.3 Vortex particle kinematics . . . . . . . . . . . . . . . 22

2.4 Background flow: Potential flow . . . . . . . . . . . . . . 23

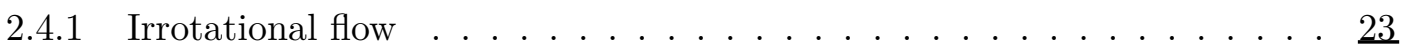


2.4.2 Boundary conditions and uniqueness of the solution . . . . . . . 24

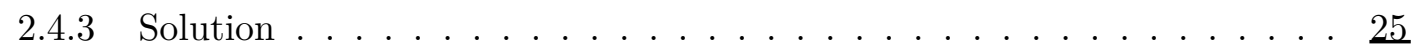

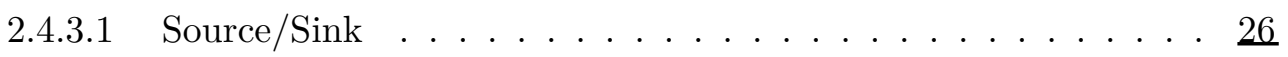

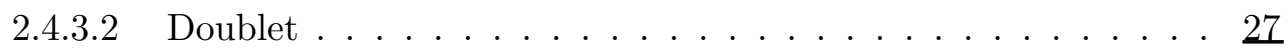

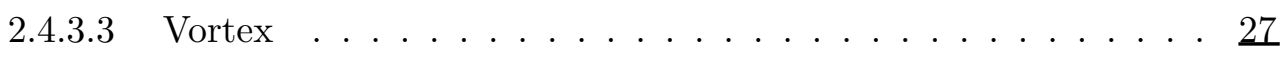

2.5 Formulation of the problem: Unsteady flow over an airfoil . . . . . . . . . 28

2.5 .1 Flow model . . . . . . . . . . . . . . . 28

2.5.1.1 Discretisation into panels . . . . . . . . . . . . 29

2.5.1.2 Flow tangency boundary condition . . . . . . . . . 29

2.5.1.3 The Kutta condition . . . . . . . . . . . . . . . . . . . 29

2.6 Numerical method: linear system . . . . . . . . . . . . . . . 31

2.6.1 Computation of Pressure and Loads . . . . . . . . . . . . 32

2.6.2 Steady $\rightarrow$ Unsteady . . . . . . . . . . . . . . 33

2.6 .3 Vortex shedding model for inviscid flow . . . . . . . . . . 33

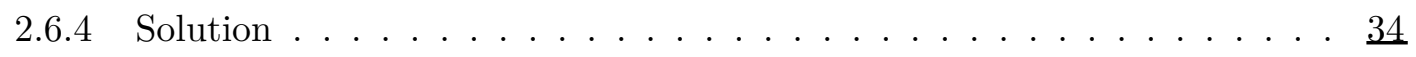

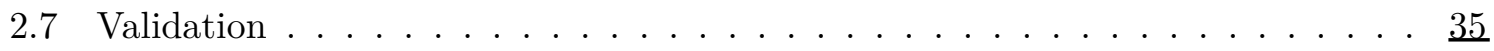

2.7 .1 Airfoil in a steady flow . . . . . . . . . . . . . 35

2.7 .2 Airfoil with an unsteady motion . . . . . . . . . . . . 37

3 Vortex Generated Turbulence $\quad \underline{41}$

3.1 Vortex methods in aeroacoustics . . . . . . . . . . . . 41

3.2 Vorticity and turbulence . . . . . . . . . . . . . . 43

3.2 .1 Sources of complexity in fluid . . . . . . . . . . . . . . . . . . . . . . .

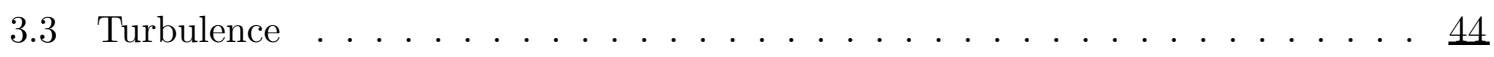

3.3 .1 Consequences of turbulence . . . . . . . . . . . . . 44

3.3.2 Measures of turbulence - Intensity . . . . . . . . . . . . . 45

3.3.3 Measures of turbulence - Scale . . . . . . . . . . . . . 46

3.3.4 Measures of turbulence - The Energy Spectrum . . . . . . . . . . . . 47

3.4 Homogeneous and Isotropic Turbulence . . . . . . . . . . . . . 47

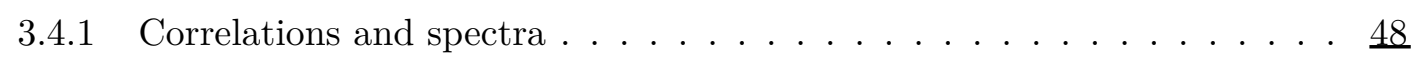

3.4 .2 One-dimensional spectra . . . . . . . . . . . . . . 49

3.5 Two-dimensional turbulence . . . . . . . . . . . . . . . . 50

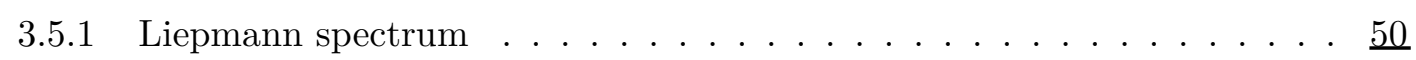

3.5 .2 von Kármán spectrum . . . . . . . . . . . . . . . . . $\underline{50}$

3.6 Representation of a Turbulent Velocity Field Using Vortex Particles . . . . . . $\underline{52}$

3.6.1 Two-dimensional system: Point vortex . . . . . . . . . . $\underline{52}$

3.6 .2 Multiple point vortices . . . . . . . . . . . . . . . . . . . . . .

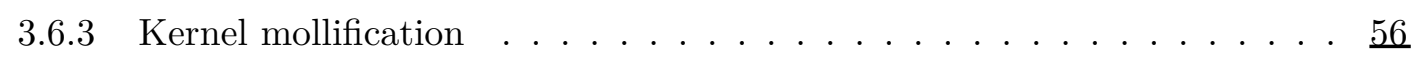

3.6.4 Single-particle statistics . . . . . . . . . . . . . . . 61

3.6.4.1 Inference . . . . . . . . . . . . . . . . 63

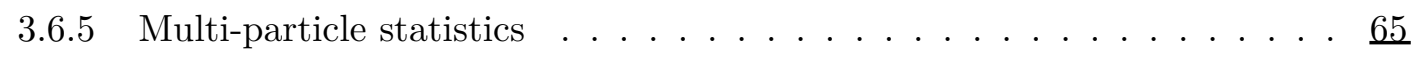

3.6.5.1 Characterisation of stochastic variables ........ 66

3.6.5.2 Choosing an appropriate distribution ......... . 66

3.6 .6 Creating realistic $2 \mathrm{D}$ turbulence . . . . . . . . . . . . . 69 
3.6.6.1 Gaussian vortices . . . . . . . . . . . 70

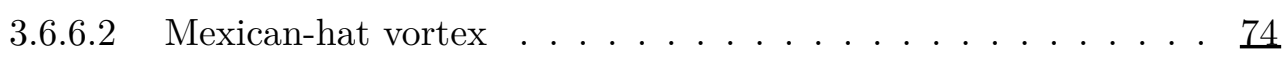

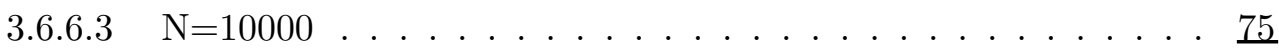

3.6.6.4 Association between vortices and turbulence . . . . . . 77

4 Sound Generation due to Turbulence $\quad \underline{91}$

4.1 Aerodynamic Sound . . . . . . . . . . . . . . . . . . . . . . 91

4.1.1 Lighthill's Acoustic Analogy . . . . . . . . . . . . . . . . . . . 92

4.1.2 Curle's equation: noise from stationary objects in low-speed flows . . . . 22

4.2 Airfoil-turbulence interaction / Leading-edge Noise . . . . . . . . . . . . . 96

4.2 .1 Steady background flow . . . . . . . . . . . . . . 96

4.2 .2 Acoustic radiation due to a single vortex . . . . . . . . . . . . 98

4.2 .2 .1 Implementation . . . . . . . . . . . . . . . . 98

4.2.3 Acoustic radiation due to a single injection point . . . . . . . . . . . 104

4.2.3.1 Vortex-sound database . . . . . . . . . . . . 105

4.2.4 Reconstruction of the velocity field . . . . . . . . . . . . . . 108

4.2 .5 Acoustic radiation due to a turbulent signal . . . . . . . . . . 110

4.2.5.1 Vortex-sound database . . . . . . . . . . . . . . 110

4.2.6 How to use the vortex-sound database? . . . . . . . . . . . . . . 114

4.2.6.1 Virtual injection of 10000 particles . . . . . . . . . . . . 114

4.2 .7 2D-3D Correction . . . . . . . . . . . . . . . . 114

4.3 Results and discussion . . . . . . . . . . . . . . . . . . . 114

4.3.1 Comparison with Amiet's analytical formulation . . . . . . . . 116

$4.3 .2 \quad$ Effect of $T u$ and $\lambda \ldots \ldots \ldots \ldots \ldots \ldots$

4.3 .3 Effect of thickness . . . . . . . . . . . . . . . . . . 119

4.3 .4 Effect of angle of attack . . . . . . . . . . . . . . . . . 119

5 Conclusion $\quad \mathbf{1 2 3}$

5.1 General conclusions . . . . . . . . . . . . . . . . . . 123

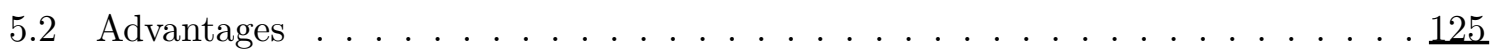

5.3 Future scope . . . . . . . . . . . . . . . . . . 125

$\begin{array}{lr}\text { References } & 129\end{array}$

Appendix A Appendix A $\quad \underline{143}$

A.1 Green's Functions and Solutions of Laplace's Equation . . . . . . . . . . . . . 143

A.1.1 Poisson's equation . . . . . . . . . . . . . . . . . . . 143

A.1.1.1 The fundamental solution . . . . . . . . . . . . 143

Appendix B Appendix B $\quad \mathbf{1 4 5}$

B.1 Spectral analysis of velocity signals of the vortex window of different lengths.$\underline{145}$

$\begin{array}{llr}\text { Appendix C Appendix C } & 147\end{array}$

C.1 Levenberg-Marquardt algorithm . . . . . . . . . . . . . . . . . . 147

C.1.1 The solution . . . . . . . . . . . . . . . 147 



\section{List of Figures}

1.1 Incidence turbulence interacting with the leading-edge of an airfoil . . . . . $\quad \underline{2}$

1.2 Distribution of studies on airfoil-turbulence interaction based on the nonexhaustive list tabulated in Table. $(1.1) \ldots \ldots \ldots \ldots$

2.1 Lagrangian and Eulerian point of view approach to describe fluid behaviour . . 18

2.2 (a) Streamlines due to source element at the origin. (b) Streamlines due to a doublet at the origin. (c) Streamlines and equipotential lines for a vortex at

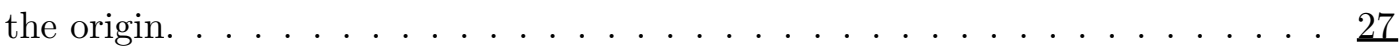

2.3 Lagrangian and Eulerian point of view approach to describe fluid behaviour . . $\underline{28}$

2.4 Panel method applied to an airfoil in unsteady flow conditions . . . . . . . . 30

2.5 Comparison of Wagner's function with the numerical predictions for a flat plate and a NACA 0001 profiles. . . . . . . . . . . . . . . . . 36

2.6 Development of circulation over chord length . . . . . . . . . . . . . . 36

2.7 Schematic of a NACA0012 airfoil at an angle of attack, $\alpha$, in a steady flow, $U$. $\underline{36}$

$2.8 C_{p}$ distributions for the NACA0012 airfoil for the indicated angles of attack . . 38

2.9 Lift coefficient $\left(C_{l}\right)$ vs angle of attack $(\alpha)$ for a NACA0012 airfoil . . . . . . 38

2.10 Reduced frequency $(k)$ vs. lift coefficient $\left(C_{l}\right)$ for a NACA0012 airfoil performing pitching motion with an amplitude of $\bar{\alpha}=2^{o} \ldots \ldots \ldots \ldots$. . . . . . . .

2.11 NACA 0012 airfoil undergoing pitching and heaving, simultaneously . . . . . 39

2.12 Unsteady lift coefficient of NACA0012 airfoil undergoing pitching and heaving motion, simultaneously . . . . . . . . . . . . . . . . . 40

3.1 Transitioning from laminar flow to turbulent one; straight, parallel grey lines are streamlines, which are everywhere parallel to the mean flow. . . . . . . . 44

3.2 Reynolds decomposition of the velocity recorded at point A . . . . . . . . 45

3.3 Basic frame $\left(\kappa_{1}, \kappa_{2}, \kappa_{3}\right)$ and local frame $\left(\tilde{\kappa}_{1}, \tilde{\kappa}_{2}, \tilde{\kappa}_{3}\right)$ in the Fourier space. The Fourier contribution $\hat{\boldsymbol{u}}(\boldsymbol{\kappa})$ is in the plane $\left(\tilde{\kappa}_{1}, \tilde{\kappa}_{2}\right)$. The orthonormal local frame, also known as the Craya-Herring frame, is defined by analogy with spherical coordinates, $\tilde{\kappa}_{3}$ is along the wavenumber $\kappa, \tilde{\kappa}_{1}$ is tangent to the meridian circle with increasing $\theta, \tilde{\kappa}_{1}$ is tangent to the parallel circle with increasing $\varphi[122] \ldots \underline{48}$

3.4 One-dimensional spectra for longitudinal, $E(\kappa)_{(11)}^{2 D(1)}$, and transverse, $E(\kappa)_{(22)}^{2 D}(1)$, component of the Liepmann and von Kármán spectra for a two-dimensional turbulence . . . . . . . . . . . . . . . . . . . . . 51

3.5 Flow field: velocity field plotted as $z$-axis for a point vortex (a) $u_{1}$, (b) $u_{2}$; and (c) two-dimensional plot of the velocity field on $x y$-plane $\ldots \ldots \ldots \ldots$ 
3.6 Flow field induced by four point vortices located at $( \pm 0.1,0)$ and $(0, \pm 0.1) \ldots$

3.7 Flow field: velocity field plotted as $z$-axis for a Gaussian (a, c, e) and Mexicanhat $(\mathrm{b}, \mathrm{d}, \mathrm{f})$ waveform vortex (a) $u_{1}$, (b) $u_{2}$; and (c) $\ldots \ldots \ldots \underline{59}$

3.8 Velocity profiles of the Gaussian and the Mexican-hat mollified kernels based on the variations in their circulation strength and radius . . . . . . . 60

3.9 single vortex moving with the freestream velocity . . . . . . . . . . . 61

3.10 Power Spectral Density (PSD) due to Gaussian waveform vortices and Mexicanhat waveform vortices (Note: $E(f)=S_{x x}(f) \ldots \ldots \ldots \ldots$

3.11 Attributes of the vortex window . . . . . . . . . . 64

3.12 Vortices arranged in UUNR configuration; green symbols indicate negative (clockwise flow), and blue symbols indicate positive (counterclockwise flow) circulation, respectively. . . . . . . . . . . . . . . 71

3.13 Vortices arranged in UNNR configuration; green symbols indicate negative (clockwise flow), and blue symbols indicate positive (counterclockwise flow) circulation, respectively. . . . . . . . . . . . . . 72

3.14 Vortices arranged in NUNR configuration; green symbols indicate negative (clockwise flow), and blue symbols indicate positive (counterclockwise flow)

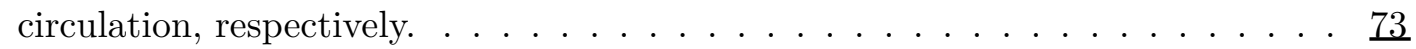

3.15 Vortices arranged in NNNR configuration; green symbols indicate negative (clockwise flow), and blue symbols indicate positive (counterclockwise flow) circulation, respectively. . . . . . . . . . . . . . 73

3.16 PDFs obtained from 300 iterations of random circulation distribution. Values are normalised by their standard deviation, and the total probability density integrates to unity . . . . . . . . . . . . . . . $\ldots 6$

3.17 Velocity spectra UUNR Gaussian Mexican-hat . . . . . . . . . . . 78

3.18 (l) Velocity fluctuations obtained at $(x, y)=(0,0)$, and $(\mathrm{r})$ longitudinal and transverse energy spectra due to $(b) N=200,(d) N=400$, and $(e) N=800$ frozen Gaussian vortices arranged in $U U N R$ configuration convecting in the streamwise direction compared with Liepmann energy spectra of two-dimensional turbulence . . . . . . . . . . . . . . . . . . 79

3.19 (l) Velocity fluctuations obtained at $(x, y)=(0,0)$, and (r) longitudinal and transverse energy spectra due to $(b) N=200,(d) N=400$, and $(e) N=800$ frozen Gaussian vortices arranged in $U N N R$ configuration convecting in the streamwise direction compared with Liepmann energy spectra of two-dimensional turbulence . . . . . . . . . . . . . . . . .

3.20 (l) Velocity fluctuations obtained at $(x, y)=(0,0)$, and $(\mathrm{r})$ longitudinal and transverse energy spectra due to $(b) N=200,(d) N=400$, and $(e) N=800$ frozen Gaussian vortices arranged in $N U N R$ configuration convecting in the streamwise direction compared with Liepmann energy spectra of two-dimensional turbulence . . . . . . . . . . . . . . . . . . . . . . 81 
3.21 (l) Velocity fluctuations obtained at $(x, y)=(0,0)$, and (r) longitudinal and transverse energy spectra due to $(b) N=200,(d) N=400$, and $(e) N=800$ frozen Gaussian vortices arranged in $N N N R$ configuration convecting in the streamwise direction compared with Liepmann energy spectra of two-dimensional

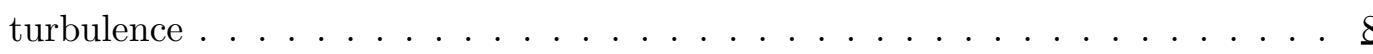

3.22 (l) Velocity fluctuations obtained at $(x, y)=(0,0)$, and $(r)$ longitudinal and transverse energy spectra due to $(b) N=200,(d) N=400$, and $(e) N=800$ frozen Mexican-hat vortices arranged in $U U N R$ configuration convecting in the streamwise direction compared with Liepmann energy spectra of two-dimensional

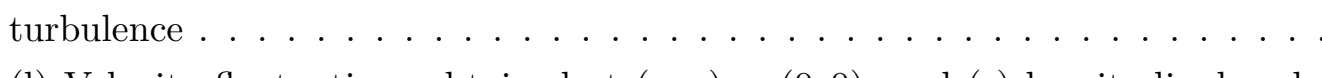

3.23 (l) Velocity fluctuations obtained at $(x, y)=(0,0)$, and $(\mathrm{r})$ longitudinal and transverse energy spectra due to $(b) N=200,(d) N=400$, and $(e) N=800$ frozen Mexican-hat vortices arranged in $U N N R$ configuration convecting in the streamwise direction compared with Liepmann energy spectra of two-dimensional turbulence . . . . . . . . . . . . . . . . .

3.24 (l) Velocity fluctuations obtained at $(x, y)=(0,0)$, and $(\mathrm{r})$ longitudinal and transverse energy spectra due to $(b) N=200,(d) N=400$, and $(e) N=800$ frozen Mexican-hat vortices arranged in $N U N R$ configuration convecting in the streamwise direction compared with Liepmann energy spectra of two-dimensional

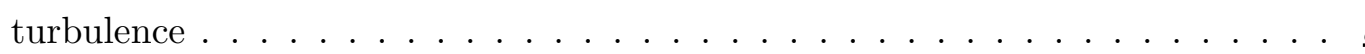

3.25 (l) Velocity fluctuations obtained at $(x, y)=(0,0)$, and $(\mathrm{r})$ longitudinal and transverse energy spectra due to $(b) N=200,(d) N=400$, and $(e) N=800$ frozen Mexican-hat vortices arranged in $N N N R$ configuration convecting in the streamwise direction compared with Liepmann energy spectra of two-dimensional turbulence . . . . . . . . . . . . . . . . .

3.26300 iterations per case, Liepmann spectra; Mexican-hat, $u_{r m s}$-lamda-gammaradius-association . . . . . . . . . . . . . . . .

3.27300 iterations per case, Liepmann spectra; Gaussian, $u_{r m s}$-lamda-gamma-radiusassociation

4.1 Monopole, dipole, and quadrupole generating waves on the surface of the water around the boat (inspired by Wagner, Hüttl, and Sagaut [157]). A person jumping up and down in the boat, producing an unsteady volume injection, thus generating a monopole wave field (top); two persons on the boat playing with a ball, exerting a force on the ball each time which results in the generation of an oscillating force which in turn generates a dipole field (middle); two individuals fighting with each other is a reasonable model for quadrupole (bottom). . . . . $\underline{93}$

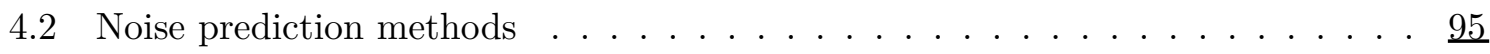

4.3 Steady background flow around NACA0012 airfoil using the panel method . . . 97

4.4 Acoustic field due to the interaction of a single vortex injected from the injection line in the streamwise direction . . . . . . . . . . . . . . . 99

4.5 single particle acoustic signature . . . . . . . . . . . . . . 101

4.6 stagnation streamline acoustic signature . . . . . . . . . . . . 102

4.7 Pressure $\Gamma$ relation $\ldots \ldots \ldots \ldots \ldots \ldots \ldots \ldots$ 
4.8 Convection of particles from a single injection point into the domain . . . . 106

4.9 Database matrix of sound pressure levels for a single injection point . . . . 107

4.10 single streamline injection: 500 particles with $\Delta t=0.001 \mathrm{~s} \ldots \ldots \ldots$

$4.11(a-b)$ spectral density of velocity induced by 10000 particles convecting in straight lines resembling streamlines with no body of influence in the domain;

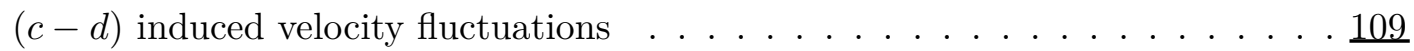

4.12 all injection points . . . . . . . . . . . . . . . . . 111

4.13 Implementation of the method . . . . . . . . . . . . . . . 112

4.14 Multidimensional matrix . . . . . . . . . . . . . . . . . . 115

4.15 Sound Pressure Level (SPL) due to 10000 vortices on NACA0012 airfoil . . . 117

4.16 Sound Pressure Level (SPL) due to 10000 vortices on NACA0010 airfoil . . . 118

4.17 SPL variations with $\mathrm{Tu}$ and $\lambda \ldots \ldots \ldots \ldots \ldots \ldots \ldots \ldots$

4.18 Sound Pressure Level (SPL) due to 10000 vortices airfoil of varying thickness .120

4.19 Sound Pressure Level (SPL) due to 10000 vortices airfoil of varying angle of attack 121

5.1 Construction and implementation of the vortex-sound database model $\ldots . .127$

B.1 spectral analysis of velocity signals of the vortex window of 25 chord lengths long 0.2 chord length wide . . . . . . . . . . . . . . . 145

B.2 spectral analysis of velocity signals of the vortex window of 50 chord lengths long 0.2 chord length wide . . . . . . . . . . . . . . . 146

B.3 spectral analysis of velocity signals of the vortex window of 100 chord lengths long 0.2 chord length wide . . . . . . . . . . . . . . . 146 


\section{List of Tables}

1.1 Selected literature on the methods to predict airfoil-turbulence interaction noise.

This is not an exhaustive list. . . . . . . . . . . . . $\underline{8}$

1.2 Selected literature on numerical methods to predict airfoil-turbulence interaction noise. This is not an exhaustive list. . . . . . . . . . . . . . . . . 11

3.1 Vortex parameters controlling the turbulence statistics . . . . . . . . 65

3.2 Special probability distribution functions $[145] \ldots \ldots \ldots \ldots$

3.3 Probability Density Functions for stochastic variables based on their characteristics discussed in section . . . . . . . . . . . . . . . 68

3.4 Vortex parameters controlling the turbulence statistics . . . . . . . . . . 70

3.5 Vortex parameters controlling the turbulence statistics . . . . . . . . . . 76

3.6 Association between vortices parameters and turbulence parameters; $\left(\mathrm{u}_{r m s}, \lambda\right.$

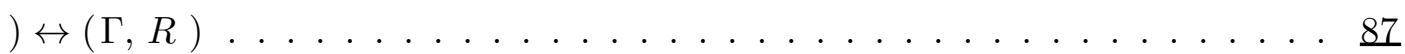

4.1 Parameters for steady-state analysis of flow around NACA0012 airfoil . . . . . $\underline{98}$

4.2 Vortex parameters for a single particle . . . . . . . . . . . . 100

4.3 Parameters involved in the interaction of 500 vortices, injecting out of the same point, with NACA0012 airfoil . . . . . . . . . . . . . . . 107

4.4 Vortex parameters controlling the turbulence statistics for streamline convection 108

4.5 Parameters involved in the interaction of 10000 vortices, injecting out of 20 points scattered uniformly on the injection line, with NACA0012 airfoil . . . 116 



\section{Abbreviations}

$\begin{array}{ll}\text { ACARE } & \text { Advisory Council for Aerospace Research in Europe } \\ \text { BEM } & \text { Boundary Element Method } \\ \text { BVI } & \text { Blade Vortex Interaction } \\ \text { CAA } & \text { Computational Aeroacoustics } \\ \text { CFD } & \text { Computational Fluid Dynamics } \\ \text { DALYS } & \text { Disability Adjusted Life Years } \\ \text { DNS } & \text { Direct Numerical Simulation } \\ \text { FFT } & \text { Fast Fourier Transform } \\ \text { FMM } & \text { Fast Multipole Method } \\ \text { FWH } & \text { Ffowcs Williams and Hawkings } \\ \text { Hi-Fi } & \text { High Fidelity } \\ \text { LBM } & \text { Lattice Boltzmann Method } \\ \text { LE } & \text { Leading Edge } \\ \text { LES } & \text { Large Eddy Simulation } \\ \text { LM } & \text { Levenberg-Marquardt } \\ \text { Lo-Fi } & \text { Low Fidelity } \\ \text { NACA } & \text { National Advisory Committee for Aeronautics } \\ \text { NNNR } & \text { Normal-Normal-Normal-Rayleigh } \\ \text { NSE } & \text { Navier-Stokes Equations } \\ \text { NUNR } & \text { Normal-Uniform-Normal-Rayleigh } \\ \text { ODE } & \text { Ordinary Differential Equation } \\ \text { PDE } & \text { Partial Differential Equation } \\ \text { PDF } & \text { Probability Density Function } \\ \text { PSD } & \text { Power Spectral Density } \\ \text { rms } & \text { root-mean-square } \\ \text { RPM } & \text { Random Particle Mesh } \\ \text { SEM } & \text { Synthetic Eddy Modelling } \\ \text { SNGR } & \text { Stochastic Noise Generation and Radiation } \\ \text { UNNR } & \text { Uniform-Normal-Normal-Rayleigh } \\ \text { UUNR } & \text { Uniform-Uniform-Normal-Rayleigh } \\ \text { VPM } & \text { Vortex Particle Method } \\ \text { VPR } & \text { Vortex Production Rate } \\ \text { WHO } & \text { World Health Organisation } \\ & \text { NA } \\ \text { NH }\end{array}$





\section{Symbols}

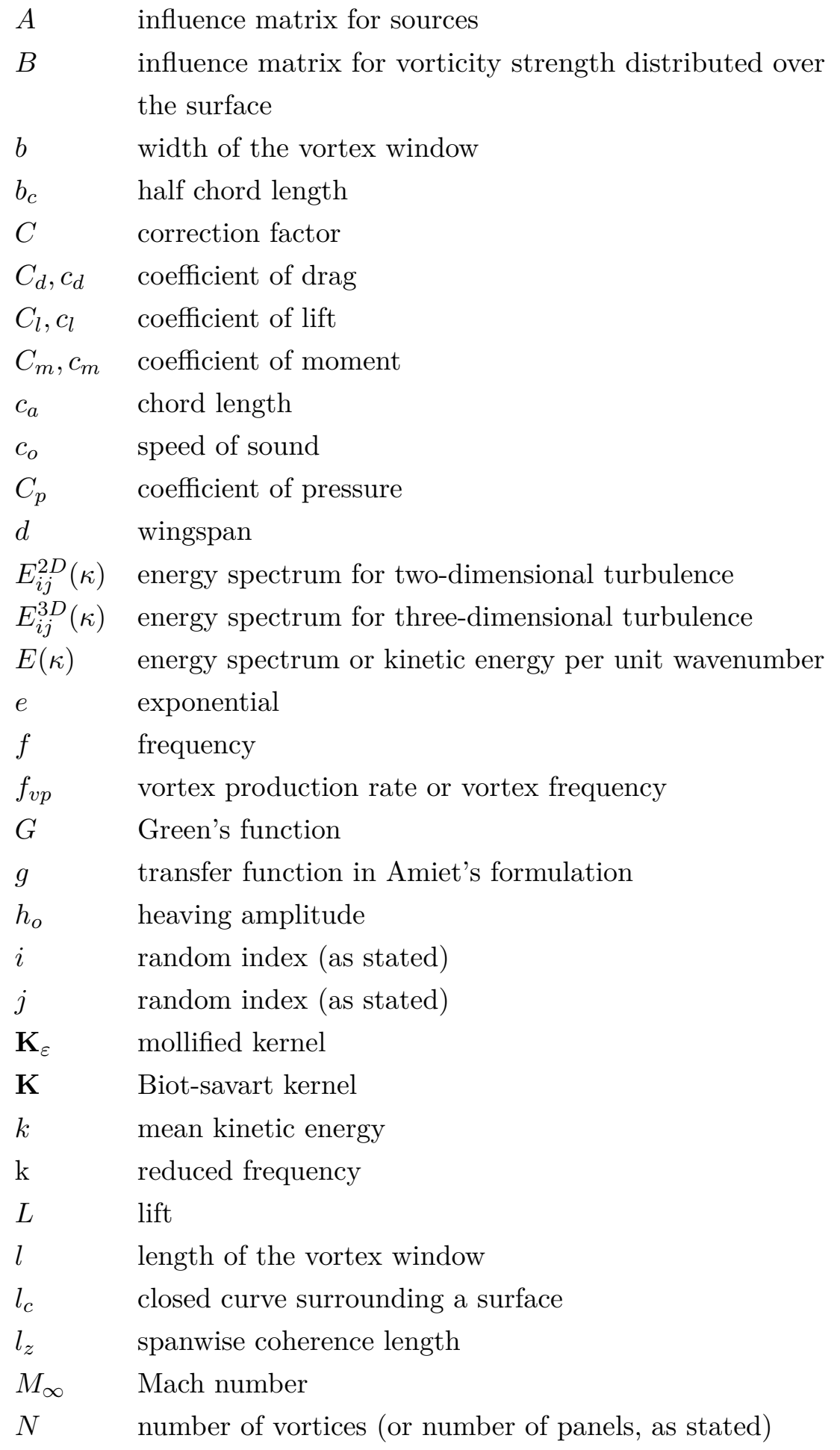




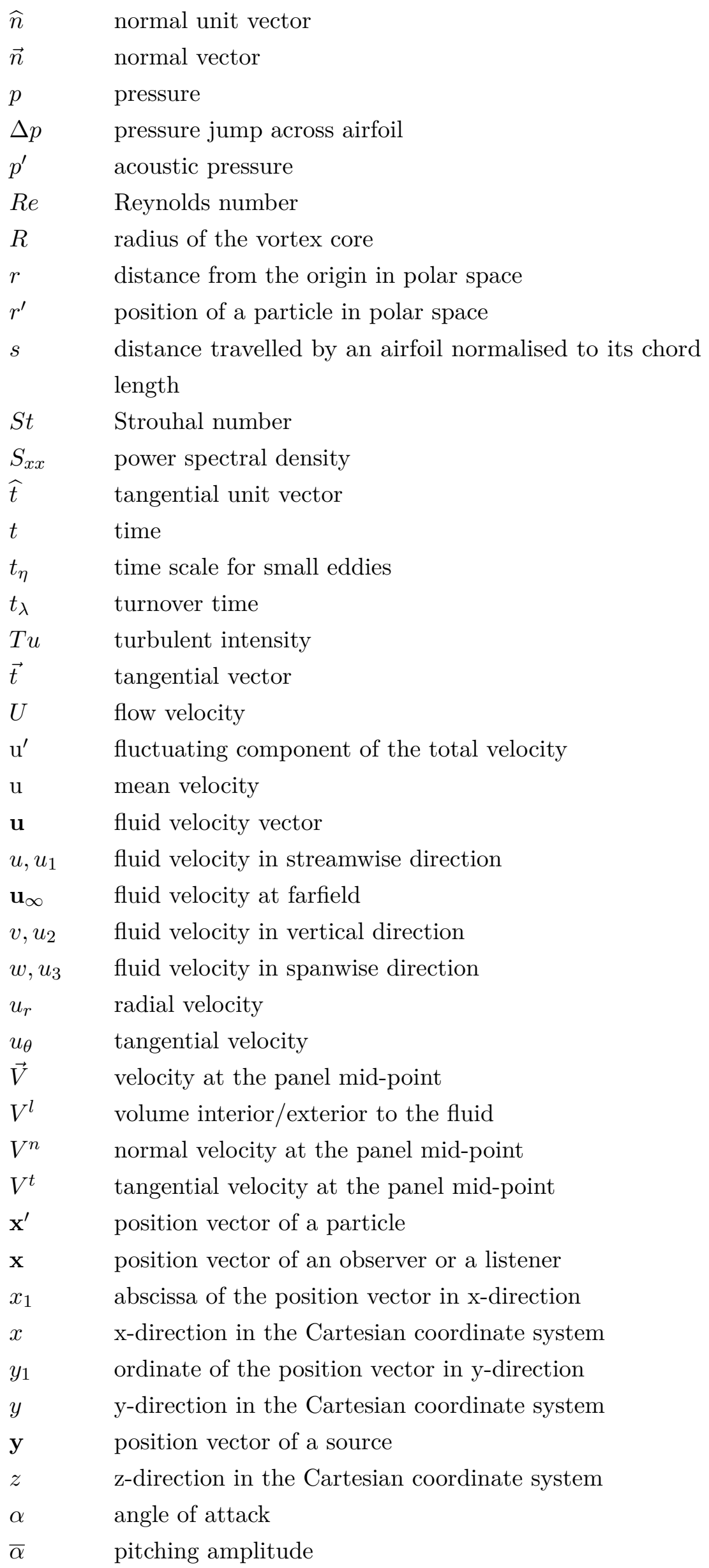


$\Gamma \quad$ circulation

$\gamma_{w} \quad$ vorticity distribution on the wake panel

$\gamma \quad$ circulation on a panel or a particle

$\Delta \quad$ Laplacian operator

$\delta_{i j} \quad$ Kronecker delta

$\epsilon \quad$ dissipation rate per unit mass

$\eta \quad$ Kolmogorov length scale

$\theta \quad$ angle (in radians) between the line from the origin to the point and the $\mathrm{x}$-axis in polar space

$\iota \quad$ iota

$\kappa_{x}, \kappa_{1} \quad$ longitudinal wavenumber for turbulence

$\kappa_{y}, \kappa_{2}$ transverse wavenumber for turbulence

$\kappa_{z}, \kappa_{3} \quad$ lateral wavenumber for turbulence

$\lambda \quad$ integral length scale

$\lambda_{v} \quad$ average distance between adjacent particle

$\mu \quad$ dynamic viscosity

$\mu_{d} \quad$ double layer potential (or doublet)

$\nu \quad$ kinematic viscosity

$\xi \quad$ coordinate of the particle in a trajectory at time $t$

$\pi \quad$ pi

$\varrho \quad$ vortex size control

$\rho_{o}, \rho \quad$ density

$\sigma_{s} \quad$ single layer potential (or source)

$\tau \quad$ time difference

$\widehat{\widehat{w_{R}}} \quad$ Fourier transform of turbulent velocity

$\phi_{i j} \quad$ spectral tensor of the velocity field

$\phi \quad$ velocity potential

$\chi \quad$ cut-off function in smoothed velocity kernel

$\psi \quad$ scalar streamfunction

$\boldsymbol{\Psi}_{\gamma} \quad$ vector streamfunction due to vorticity strength on the body surface

$\boldsymbol{\Psi}_{\infty} \quad$ vector streamfunction at farfield

$\boldsymbol{\Psi}_{\omega} \quad$ vector streamfunction due to vorticity

$\boldsymbol{\Psi} \quad$ vector streamfunction

$\omega \quad$ vorticity (z-component)

$\mathcal{D} \quad$ computational domain

$\infty \quad$ infinity

$\mathcal{L} \quad$ linear elliptic operator

$\mathcal{P} \quad$ stress tensor

$\mathrm{R}_{i j} \quad$ correlation function

$\mathbb{R} \quad$ positive real numbers

$\mathcal{S} \quad$ sphere in Fourier space

$\star \quad$ convolution 
$\eta \quad$ used to address an idea/hypothesis

$\times \quad$ cross product

- $\quad$ dot product or multiplication (if stated)

$\forall \quad$ for all

$\propto \quad$ proportional to 


\title{
1
}

\section{Introduction}

\author{
Sparsh Sharma, Ennes Sarradj, and Heiko Schmidt. "Unsteady Lift due to the Interaction \\ of Incidence Turbulence with an Airfoil". In:Fortschritte der Akustik - DAGA 2017 \\ 43.Deutsche Jahrestagung für Akustik: 06.-09. März 2017 in Kiel. Kiel, Germany, 2017
}

Turbulence is everywhere, yet it is one of the most challenging problems for physicists to understand. The mathematics of turbulence would at first appear to present an uncomplicated event. The Navier-Stokes equations, a set of nonlinear partial differential equations, have been used to describe fluid flow and they take into account properties of the fluid such as its density and viscosity, along with any forces acting on it. Moreover, for all practical purposes, the nonlinearity due to the convective acceleration makes this equation impossible to solve. If researchers can find a way to capture this chaos in an equation, it will become easier to understand this universal event from ocean currents to how gases move throughout galaxies. In the physical world, the interaction of turbulence with engineering designs such as turbofan engines, submarine propellers and wind turbines results in the generation of noise. Particularly in aircraft engines, the interaction of atmospheric flow with the blades of the front fan which further convects downstream and interact with stator blades or vanes generates broadband noise, which can be a significant portion of the overall noise. Furthermore, the current trend of high bypass ratio engines with relatively shorter ducts restrains the usage of sound-absorbing liners by reducing the area required. The noise emission from the fan mainly has two components: tonal noise and broadband noise. The displacement effect of an immersed body in motion and the unsteady aerodynamic loads on the body surface generate pressure fluctuations that are radiated as sound. This kind of noise is deterministic and relatively easy to extract from aerodynamic simulations because the required resolution in space and time to predict the acoustics is like the demands from the aerodynamic computations. If the surfaces move at speeds comparable to the speed of sound or there is an interaction between a rotor and a stator wake, these tonal noise components can be dominant. At low Mach numbers, the interaction of airfoil with the turbulent inflow introduces an unsteady pressure loading on its surface, which produces noise. This is referred to as "leading-edge noise", and it is gaining a 
renowned interest in the research community lately. Due to considerable efforts in tonal noise reduction, broadband noise reduction at the source remains a topic of significant interest for the industry and the research community in general. The Advisory Council for Aerospace Research in Europe (ACARE) has set the goal to reduce the noise emission of flying aircraft by $65 \%$ relative to the capabilities of typical new aircraft in 2000 . Studies to understand the generation of broadband noise, which is indeed difficult to predict and to reduce because of its random nature and broad frequency content, is thus beneficial to predict and minimise the noise generated by it.

\subsection{Airfoil turbulence interaction noise}

By definition, unsteady flow is one where the flow field variables at any point are changing with time, which means all the aerodynamic parameters fluctuate with time too and are certainly the inhibitors of all the disturbances which causes the generation of noise from an airfoil. As stated, turbulence noise exists almost every time; consequently, aerodynamic noise is usually a broadband noise sometimes augmented by narrow tonal components coming from impulsive noise sources.

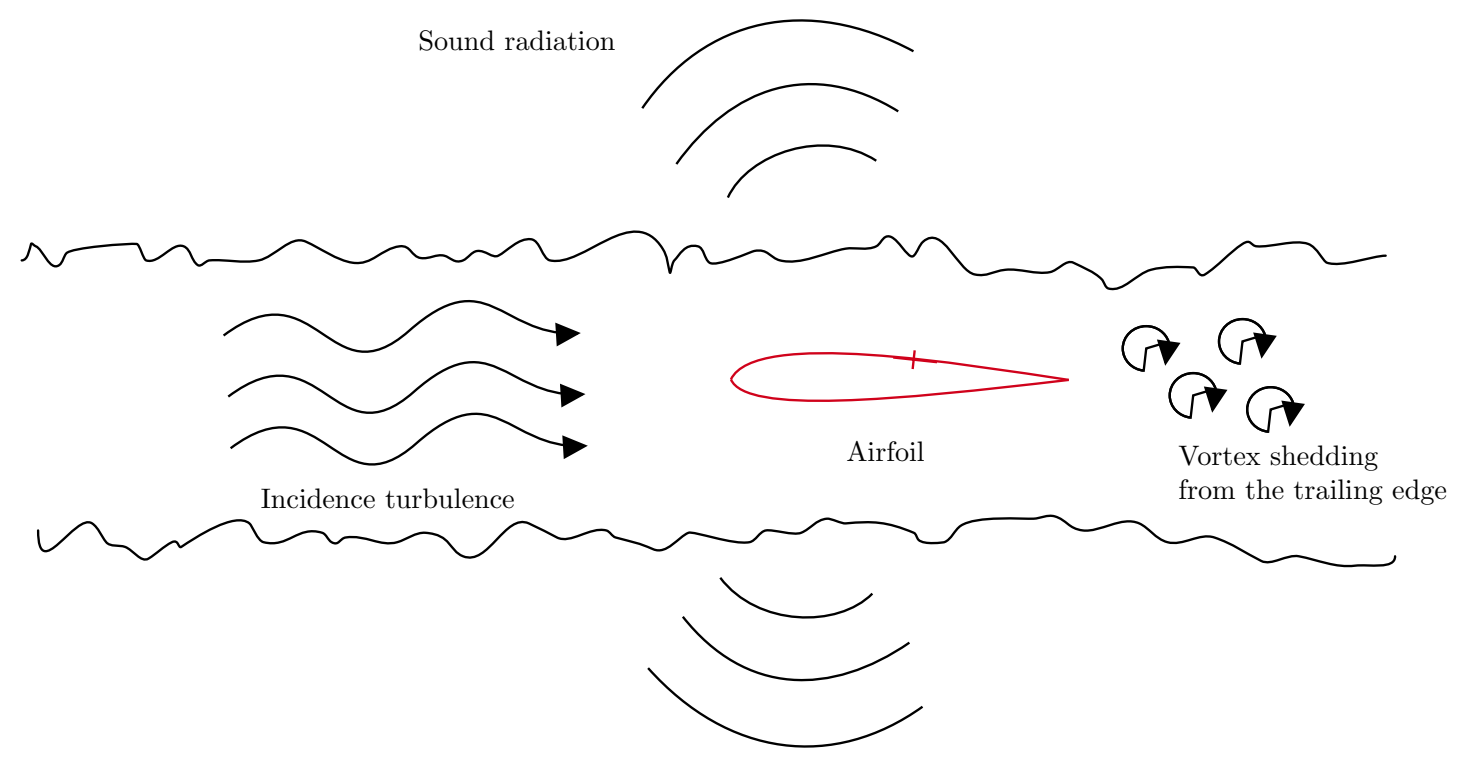

Figure 1.1: Incidence turbulence interacting with the leading-edge of an airfoil

Fig. (1.1) illustrates the noise generation mechanism due to the interaction of incidence turbulence with the leading-edge of an airfoil. Airfoil-turbulence interaction is, however, one of three possible mechanisms which describe the generation of the broadband component of the frequency spectrum ${ }_{-}^{1}$. Out of the remaining two mechanisms, the random force fluctuation on a surface in a moving flow arising from a turbulent boundary layer is the first one. Another form in which the fluctuating force appears due to vorticity shedding from the surface of a body moving in a flow [1].

\footnotetext{
${ }^{1} \mathrm{~A}$ frequency spectrum indicates the general nature of fan noise
} 


\subsubsection{Unsteady aerodynamics}

The early research in the field of unsteady aerodynamics has been an excellent source of motivation for the discussion of acoustic radiation from a body immersed in a turbulent flow or a body making a nonuniform motion in the flow field. A detailed discussion of unsteady aerodynamics is essential to understand the underlying phenomena which govern the pressure fluctuations over the surface of a body immersed in a fluid flow. The fundamental equations describing unsteady viscous flow processes are the Navier-stokes equations which started to become amenable to solution recently due to the advent of powerful high-speed computers. However, there were times when physicist did not have such powerful resources to calculate such nonlinear equations, and yet the concept of the boundary layer was introduced by Prandtl [2] in 1904. Later in 1924, Prandtl [3] suggested to neglect the influence of viscosity while treating the problem of incompressible flow past an oscillating airfoil, and thus to take the Laplace equation as governing equation. It was pointed out that every change in the lift must be accompanied by the detachment of a vortex from the airfoil's trailing edge. An important parameter, reduced frequency $\mathrm{k}$, was introduced by Birnbaum [4] to characterise the fluid dynamic phenomena for periodic oscillations of the airfoil or the flow,

$$
\mathrm{k}=\frac{\omega c_{a}}{U}
$$

where $\omega$ is the angular frequency, $c_{a}$ is the chord length and $U$ the flow speed. This parameter is a measure of unsteadiness. When an oscillating airfoil sheds a vortical wake with a particular wavelength, the reduced frequency compares this wavelength with the airfoil chord because during one oscillation a vortex shed from the trailing edge travels the distance $U / \omega$. This means, the higher the reduced frequency, the smaller the wavelength.

i) Steady state aerodynamics, $\mathrm{k}=0$

ii) Quasi-steady aerodynamics, $0 \leq \mathrm{k} \leq 0.05$

iii) Unsteady aerodynamics, $\mathrm{k}>0.05$ ( $\mathrm{k}>0.2$ is considered highly unsteady)

Birnbaum reduced the problem to an integral equation employing theorems on vortex motion while Wagner [5] obtained an integral equation by way of formulating the boundary problem for the velocity potential. Birnbaum's formulation (in common with the formulation of more general problems utilising the acceleration potential) possesses the disadvantage of leading to an integral equation with a considerably more complicated kernel than in the velocity potential formulation of the same problem. Birnbaum obtained solutions of this integral equation using numerical methods restricted, however, to a far too small range of values of important reduced frequency.

The basis of these pioneer researches in unsteady aerodynamics is the indicial response method. This is the response of the aerodynamic flow field to a step change in a set of defined boundary conditions such as a step-change in airfoil angle of attack, pitch rate about some axis, or in control surface deflection (such as a tab or flap). If the indicial response can be computed, then the response to an arbitrary motion of the airfoil or control surface can be found by Duhamel superposition [6]. If the linearity of the physics over the required range of conditions can be 
justified, then the advantage of the indicial method is a tremendous saving in computational cost over performing separate flow field calculations. A particularly useful application of the indicial method is in the calculation of gust-induced air loads. The unsteady effects produced on airfoil arise primarily because of the vertical velocity between the disturbance (the gust field) and the airfoil surface. In linear theory, this component is used to satisfy the boundary conditions of flow tangency on the airfoil surface $[\underline{7}, \underline{8}$.

In Wagner's work, attention is focused on transient problems for which series developments solve the integral equation of the problem. Both authors deal only with the motion of a rigid straight-line profile. Wagner analytically predicted a function that concerns the growth of circulation or lift about an airfoil at a small fixed angle of attack starting impulsively from rest to a uniform velocity. He did not derive an explicit analytic expression for his function. The function depends on a non-dimensional parameter, $s$, distance traversed in terms of half chord and stands affirm for smaller values of $s$. In 1935, Theodorsen [9] published his renowned work establishing the numerical method used for calculating the response of the body to incoming instability and flutter. Theodersen function describes the lift due to circulation about an airfoil oscillating sinusoidally and moving with a uniform velocity. The method was based on potential theory and Kutta-condition. The solution is of a simple form and is expressed by means of an auxiliary parameter $\mathrm{k}$, reduced frequency. The unsteady lift force in attached flow is described as the sum of two contributions: a non-circulatory and a circulatory one. The non-circulatory lift, or added mass term, represents the lift force that would arise on the airfoil in a non-circulatory flow due to the reaction of the fluid accelerated with the airfoil motion; the non-circulatory term has no dependency on time, and only depends on the instantaneous acceleration of the fluid around the airfoil. The circulatory lift, on the contrary, carries a memory effect, which originates from the vorticity shed into the wake to compensate the change of circulation around the airfoil, as governed by Kelvin's theorem on conservation of circulation. Larger oscillations were not accounted for since the sole attempt was to specify one or more conditions of instability. Since the treatment was restricted to primary effects, certainly, no type or shape of airfoil was chosen for this work. Following, Küssner [10] predicted the formulae of lift and moment for an airfoil entering a sharp-edged gust. He verified his work experimentally, however, could not prove it theoretically because of an error in his fundamental equation for waves progressing over the airfoil. It is to be noted that Wagner and Theodersen induced the nonuniformity into the domain by making the body perform nonuniform motions with respect to time, whereas, Küssner was among the first ones who talked about the body response to the incoming instabilities in the flow. Lift for a two-dimensional thin airfoil (for the cases studied by Wagner, Theodersen, and Küssner) moving in a nonuniform flow is given by the form,

$$
L \propto f(\mathrm{k})
$$

where $L$ is lift, and $\mathrm{f}(\mathrm{k})$ is a function which specifies the nonuniform motion of the airfoil. It was Kàrmàn and Sears [11], who formulated the airfoil theory of nonuniform motion more accessible by showing the physical significance of the various steps of the mathematical deductions and eliminating unnecessary mathematical complications. Nevertheless, they summed up the entire advancements in the unsteady airfoil theory, discussed previously, into 
one simple, less complicated manner to make it more accessible for practical applications. The circulation theory of airfoil was used to show the mechanism by which a wake of vorticity is produced in nonuniform motion. The formulae for lift and moment were derived for a two-dimensional thin airfoil where the movement of airfoil normal to its mean path is small. The formulation did not account for thick airfoil (thickness $>12 \%$ ). In general, the airfoil is represented as a vortex sheet, and a starting vortex develops just after the impulsive motion to conserve the moment of momentum. Since the total circulation around the airfoil is timedependent in nonuniform motion, the airfoil is bound to leave a vortex trail behind itself, and the influence of this wake now must be taken care of. Karman \& Sears approach was based on the idea that the momentum per unit span of the system can be expressed by the sum of the momentum of the vortex pairs, which constitute the system. Moreover, it was assumed that all the vortices are located along the $x$-axis. The total lift and moment expressions (comprised of three components namely apparent mass, quasi-steady, and wake induced) are then applied to the case of an oscillating airfoil (Theodersen, and Wagner) and on airfoil entering sharp-edged gust (Küssner). The theory fails to analyse the behaviour of a thick airfoil, an airfoil in large-amplitude motion with deforming vortex wakes, and manoeuvring low-aspect-ratio wings with leading-edge separation. In another paper [12], Sears used the same methodology to describe the airfoil behaviour when passing through a vertical gust pattern having a sinusoidal distribution of intensity. The lift was determined as a function of reduced frequency and shown that it acts at the quarter chord point of the airfoil always.

Following the trend, Liepmann [13] proposed the lift response of a wing with a finite span moving in a turbulent field. His study was based on the statistical approach for defining turbulence. The concepts of thin-wing theory and homogeneous turbulence, the mean square lift is expressed in terms of a generalised aerodynamic admittance and one component of the power spectrum tensor of turbulence. Inspired by this work, Ribner [14] extended this approach to a swept-back wing assuming it a lifting line.

The description of the early research in the field of unsteady aerodynamics so far paves the way for the discussion of acoustic radiation from a body immersed in a turbulent flow or a body performing a nonuniform motion in the flow field.

\subsubsection{Sound radiation development}

The original theoretical and mathematical description of sound generated aerodynamically was given by Lighthill $[15,16]$. Earlier work, before Lighthill's contribution, on the sound generated aerodynamically is almost concerned with frequency. However, the evolution of a general procedure for estimating the intensity of the sound produced in terms of the fluid flow was perhaps the only relevant question that could not get the attention of physicists before Lighthill, or in other words, the acoustic power was being related to the operation conditions, yet a study of its relation to the actual flow was not attempted. Following are the salient points of the theory:

i) There is no back-reaction which means that sound produced is so weak relative to the motions producing it that it does not affect the flow field. 
ii) It applies only if the sound radiated into free space, neglecting all the effects of reflexion, diffraction, absorption or scattering by solid bodies.

iii) Sound radiation estimates refer only to the energy which escapes from the flow as sound.

iv) It applies only to low-speed subsonic flow $\left(M_{\infty} \leq 3.0\right)$.

Curle [17] extended Lighthill's general theory of aerodynamic sound to incorporate the influence of solid boundaries upon the sound field. This influence is twofold, namely (i) reflection and diffraction of the sound waves at the solid boundaries, and (ii) a resultant dipole field at the solid boundaries which are the limits of Lighthill's quadrupole distribution. In the present work, the broadband dipole noise generated by the induced fluctuating pressure on the surface of a stationary body immersed in the flow is estimated by the Curle's formulation.

The three main methods - analytical, experimental, and numerical, are widely used to understand and estimate this mechanism. It is highly suggested to briefly describe these techniques before reporting the development and implementation of the proposed mathematical model. An overview of the selected literature on the methods to predict leading-edge noise is given in Table. (1.1) from which the numerical studies are further extracted in Table. (1.2).

\subsubsection{Analytical methods}

In the early sixties, the advent of jet engines attracted many acousticians, Doak [18] in 1960 published his work on acoustic radiation from a body immersed in a turbulent flow field. The attention was given primarily to the radiation from the surface of the body. The method revolves around Green's function; generally, pressure fluctuations at the surface, in particular, are measured and used with Green's function to estimate the acoustic radiation from the surface of the body.

Sharland [1] came up with his formulation of quantitative expressions for the sources of broadband noise for axial fans. He described the noise generation from an airfoil by at least three ways:

i) Due to the random pressure fluctuations on the surface of the body arising due to turbulent boundary layer.

ii) Due to periodic vorticity shedding from a body which induces a corresponding change in the circulation and hence produces local fluctuations of lift.

iii) Finally, if a body is immersed in a turbulent flow field, the local incidence at each point on the surface is determined by the resultant of the mean velocity and the perturbation velocity.

The acoustic radiation from a body immersed in a flow field has been analysed by using the analogy of dipole radiation $[17,18]$ which can be expressed in terms of the distribution of fluctuation pressure over the surface. The highlight of his work was that he could come up with a term for radiated acoustic energy in terms of fluctuating lift force, from a blade or more specifically a flat plate immersed in the flow field with and without turbulence, and the parameters on which the source strength depends. It has also been demonstrated that intake 
turbulence can have a disastrous effect on the broadband noise produced. It was, therefore, essential and mandatory for his work to have the incoming flow as smooth as possible.

One of the most remarkable contributions in the field was from Amiet who published numerous papers in the 1970s. In one of the papers [19], a theoretical expression for the far-field acoustic power spectral density (PSD) in a subsonic turbulent stream is given in terms of quantities characteristics of turbulence. The method was inspired by the theories put forward by Kirchhoff [20] and Curle [17], which say that a fluctuating lift should result in the generation of sound. Analytical expressions for the time-dependent pressure jump distribution, $\Delta p$, across a flat plate airfoil of infinite span entering a sinusoidal gust form in frozen turbulence was derived,

$$
\Delta p(x, y, t)=2 \pi \rho_{o} U b_{c} \iint_{-\infty}^{\infty} \widehat{\widehat{w_{R}}}\left(\kappa_{x}, \kappa_{y}\right) g\left(x, \kappa_{x}, \kappa_{y}\right) e^{i\left(\kappa_{y} y-\kappa_{x} U t\right)} d \kappa_{x} d \kappa_{y}
$$

Atassi and Hamad [21] considered the three-dimensional rectilinear cascade model subject to a rotor wake or secondary flow disturbance. The work followed very closely the theory developed by Goldstein [22].

A theoretical model by Myers and Kerschen [23] considered the combined effects of airfoil camber and incidence angle on the sound field generated by interaction with high-frequency gusts. The developed analytical model accounted for the compressible high-frequency interaction between a gust convected by a subsonic mean flow, and a cambered airfoil at non-zero incidence. They highlighted the mean flow distortion effects due to the airfoil angle of attack and camber.

Gershfeld [24] developed a correction factor accounting for thickness in Amiet's flat plate model. He used acoustic analogies and shape-dependent Green's function to predict the lifting dipole sound from the leading-edge diffraction of homogeneous turbulence by a thick, symmetric foil at a zero angle of attack to the mean flow. The predicted spectra obtained from from Amiet's formula with Gershfeld's correction factor agreed well with Paterson and Amiet [25] measured data at low speeds.

Posson, Moreau, and Roger [26] extended Glegg's [27] analytical model of the aeroacoustic response of a rectilinear cascade of flat-plate blades to three-dimensional incident vortical gusts, to the prediction of the noise generated by a three-dimensional annular blade-row.

More recently, Ayton and Peake [28] presented a theoretical model to predict the far-field sound generated by high-frequency gust-aerofoil interaction in steady parallel shear flow, including the effects of aerofoil thickness. The approach was to use asymptotic analysis of the Euler equations linearised about steady parallel shear flow, in the limits of high frequency and small, but non-zero, aerofoil thickness and Mach number.

\subsubsection{Experimental methods}

The evolution of turbulent flow is very complex. Turbulent flow appear highly disorganised with the structure at all scales. Signals appear unpredictable in their detailed behaviour. The origin of air turbulence is some form of instability. In the atmosphere, this could be either a thermal instability or mechanical instability, like wind shear or flow over obstacles. In wind tunnels the turbulence can be created, for example, by a grid, where mechanical instability produces a multitude of vortices, not unlike von Kármán vortex streets. The turbulence created in this way is roughly of the scale of the grid producing it and is being continuously 
Table 1.1: Selected literature on the methods to predict airfoil-turbulence interaction noise. This is not an exhaustive list.

\begin{tabular}{|c|c|c|c|c|c|}
\hline source & year & ana $_{-}^{2}$. & $\exp _{-}^{3}$. & num $_{-}^{4}$. & $\mathrm{emp}^{5}$. \\
\hline Doak [18] & 1960 & $\mathbf{\square}$ & & & \\
\hline Sharland $[1]$ & 1964 & $\mathbf{\square}$ & & & \\
\hline Mugridge [30] & 1971 & $\mathbf{\square}$ & & & \\
\hline Dean $[29]-$ & 1971 & & घ & & \\
\hline Amiet [19] & 1975 & $\mathbf{\square}$ & & & \\
\hline Fink $[3 \overline{1,32}]$ & 1975 & & $\mathbf{\square}$ & & \\
\hline Atassi and Hamad [21] & 1981 & $\square$ & & & \\
\hline Brooks, Pope, and Marcolini [33] & 1989 & & $\mathbf{\square}$ & & $\mathbf{\square}$ \\
\hline Bechara and Bailly [34] & 1994 & & & $\square$ & \\
\hline Gennaretti, Luceri, and Morino [35] & 1997 & & & $\mathbf{\square}$ & \\
\hline Guidati et al. [36] & 1997 & & 口 & $\mathbf{\square}$ & \\
\hline Myers and Kerschen [23] & 1997 & $\mathbf{\square}$ & & & \\
\hline Bailly and Juvé [37] & 1998 & & & $\mathbf{\square}$ & \\
\hline Lockard and Morris [38] & 1998 & & & $\mathbf{\square}$ & \\
\hline Grace [39] $\quad-$ & 2000 & & & $\mathbf{\square}$ & \\
\hline Casper and Farassat [40] & 2003 & 口 & 口 & & \\
\hline Moriarty [41] & 2004 & & & & 口 \\
\hline Gershfeld [24] & 2004 & $\mathbf{\square}$ & & & \\
\hline Moreau, Roger, and Jurdic [42] & 2005 & & 口 & & $\mathbf{a}$ \\
\hline Agarwal and Morris [43] - & 2006 & & & 口 & \\
\hline Gennaretti and Bernardini [44] & 2007 & & & $\mathbf{\square}$ & \\
\hline Zhou and Joseph [45] & 2007 & & & $\mathbf{\square}$ & \\
\hline Ewert [46] - & 2007 & & & - & \\
\hline Lockard and Choudhari [47] & 2009 & & & $\mathbf{\square}$ & \\
\hline Posson, Moreau, and Roger $[26]$ & 2010 & - & & & \\
\hline Devenport, Staubs, and Glegg [48] & 2010 & & 口 & & \\
\hline Glegg and Devenport [49] $\quad$ - & 2010 & & & $\mathbf{\square}$ & \\
\hline Casalino and Barbarino $[50]$ & 2010 & & & $\mathbf{\square}$ & \\
\hline Hutcheson, Brooks, and Stead [51] & 2011 & & $\mathbf{\square}$ & & \\
\hline Geyer, Sarradj, and Hobracht $[\overline{52}]$ & 2012 & & $\mathbf{\square}$ & & \\
\hline Dieste and Gabard [53] & 2012 & & & $\mathbf{\square}$ & \\
\hline Ayton and Peake $[28]$ & 2015 & $\mathbf{\square}$ & & & \\
\hline Kim and Haeri $[54]$ & 2015 & & & $\mathbf{\square}$ & \\
\hline Miotto et al. $[55]$ & 2017 & & & $\mathbf{\square}$ & \\
\hline Gea-Aguilera, Gill, and Zhang [56] & 2017 & & & $\mathbf{\square}$ & \\
\hline
\end{tabular}

${ }^{2}$ analytical

${ }^{3}$ experimental

${ }^{4}$ numerical

${ }^{5}$ empirical 
broken down through the action of inertial and viscous forces into smaller and smaller vortices. The experimental studies are the backbone for the development of any mathematical model in physics, and the same applies to the problem of leading-edge noise. Early studies reported by Dean [29] included surface loading data from pressure transducers on an airfoil at its quarter-chord. It was assumed that all pressure fluctuations at a given point on the airfoil are caused by fluctuating incidence. The work included measurements of upstream turbulence spectra, loading spectra at the airfoil quarter-chord, and far-field acoustic pressure spectra.

Fink [31, 32] conducted tests in an acoustic wind tunnel to determine surface pressure spectra and far-field noise caused by turbulence impinging on an airfoil and turbulence convected past a sharp trailing edge.

In the investigations of Brooks, Pope, and Marcolini [33] published in 1989, five noise generation mechanisms were considered. The acoustic method takes into account the sound sources from (i) the interaction of turbulent boundary layer with trailing edge, (ii) separation-stall, (iii) laminar boundary layer vortex shedding, (iv) tip vortex formation, and (v) trailing edge bluntness vortex shedding. The result of the investigation is an empirically reliable method for the calculation of the sound emission of a flow-around wing and thus also a rotating blade of a turbomachine. The developed sound prediction method can be described as semi-empirical since it is based on already existing theoretical considerations as well as on the evaluation of extensive measurement data.

Guidati et al. [36] investigated the influence of airfoil shape on the generation of inflowturbulence noise. The experimental findings revealed that the noise generation is strongly dependent on the airfoil shapes besides the airfoil thickness. A numerical model based on Green's function and implemented through the boundary element method was validated using these experiments.

Casper et al. [40] predicted the acoustic radiation from a NACA 0015 airfoil in the time domain by a solution of the Ffowcs Williams-Hawkings (FW-H) equation. The experimentally measured surface pressure on the airfoil immersed in grid-generated turbulence was used as an input for the numerical analysis.

Moreau, Roger, and Jurdic [42] also studied the turbulence-interaction noise experimentally using grid-generated turbulence in an open jet anechoic chamber. In the tested range, the angle of attack plays no critical role. However, an increasing airfoil thickness is found to reduce the turbulence-interaction noise significantly. They extended the original statistical model proposed by Amiet to describe turbulence-interaction noise, valid for parallel gusts on flat plates in the midspan plane, to general three-dimensional supercritical and subcritical gusts. Devenport, Staubs, and Glegg [48] conducted another similar experimental study in 2010, in which the effect of airfoil geometry on the noise generation was studied. The airfoils included variations in the chord, thickness and camber. It was found that thicker airfoils generate significantly less noise at high frequencies.

More recently, Hutcheson, Brooks, and Stead [51] conducted a detailed study on airfoil leadingedge noise, analysing the influence of the parameters of the incident turbulence (turbulence intensity, integral length scale), airfoil geometry (thickness and chord length), angle of attack and flow speed on the generation of leading-edge noise at different model airfoils and flat plates. 


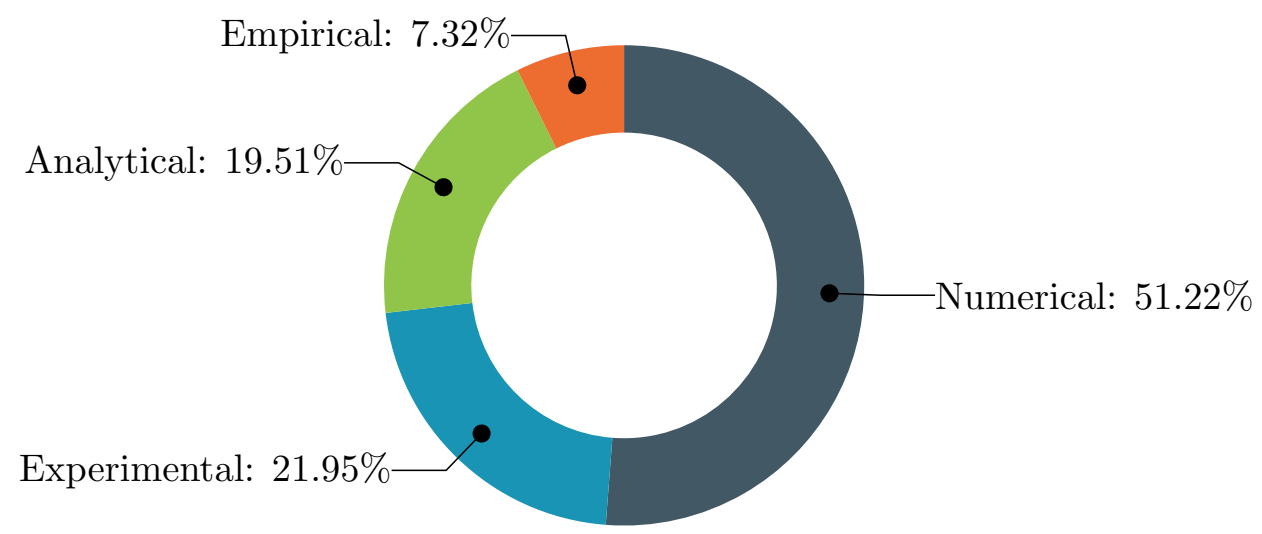

Figure 1.2: Distribution of studies on airfoil-turbulence interaction based on the non-exhaustive list tabulated in Table. (1.1)

The homogeneous and nearly anisotropic turbulence was generated using two different grids, leading to turbulence intensities in the order of $5 \%$ to $8 \%$.

In a recent study, Geyer, Sarradj, and Hobracht [52] performed constant temperature measurements both in isotropic homogeneous turbulence as well as in anisotropic inhomogeneous turbulence. The resulting data were then used to calculate turbulence interaction noise using Amiet's flat-plate model on a flat plate and a NACA 0012 airfoil.

\subsubsection{Numerical methods}

Fig. (1.2) shows the results of the survey of studies in the field of leading-edge noise. From the pie chart, it is clear that the majority of work is carried out using numerical methods which depends entirely on the usage of computers. The advent of high-performance computing platforms has enabled the scientific community to operate massive supercomputers to solve the complex Navier-stokes equations. Computational Fluid Dynamics (CFD) method has a high fidelity in predicting the airfoil-turbulence interaction, and its computational cost limits its applications at conceptual and preliminary design stages of an aircraft or a turbomachine. Nevertheless, the state-of-art High-Fidelity (Hi-Fi) numerical methods like Large Eddy Simulations (LES) are widely accepted hybrid techniques whereas methods like Direct Numerical Simulations (DNS) and Lattice Boltzmann method are considered as the direct approach in computational aeroacoustics.

\subsubsection{Euler approach}

Euler approach demands a mesh around the body which extends to a finite region to build a computational domain. The governing equations are then solved on the mesh points in this domain for pressure, density, and temperature using methods like finite difference and finite volume. Bechara and Bailly [34] split the approach to obtain the equations governing the acoustic field. Mean and turbulent components and acoustic variables are defined separately, thus defining a stochastic noise generation and radiation (SNGR) model. Later in 1998, Bailly and Juvé [37] used a similar approach to model acoustic propagation problems by linearising two-dimensional Euler's equations around a stationary mean flow. The final goal of their work 
Table 1.2: Selected literature on numerical methods to predict airfoil-turbulence interaction noise. This is not an exhaustive list.

\begin{tabular}{|c|c|c|c|c|c|}
\hline source & year & Euler & Lagrangian & frequency & time \\
\hline Bechara and Bailly [34] & 1994 & 口 & & & \\
\hline Gennaretti, Luceri, and Morino [35] & 1997 & & 口 & & [ \\
\hline Guidati et al. [36] & 1997 & & $\boldsymbol{\square}$ & [ & \\
\hline Bailly and Juvé [37] & 1998 & $\mathbf{\square}$ & & & \\
\hline Lockard and Morris [38] & 1998 & $\mathbf{\square}$ & & & \\
\hline Grace [39] & 2000 & & 口 & & 口 \\
\hline Agarwā and Morris [43] & 2006 & & $\mathbf{\square}$ & $\mathbf{\square}$ & \\
\hline Gennaretti and Bernardini [44] & 2007 & & $\mathbf{\square}$ & & $\mathbf{\square}$ \\
\hline Zhou and Joseph [45] & 2007 & & & [ & \\
\hline Ewert $[46]$ & 2008 & [ & & & \\
\hline Lockard and Choudhari [47] & 2009 & $\mathbf{\square}$ & & 口 & \\
\hline Glegg and Devenport [49] & 2010 & & $\mathbf{\square}$ & ם & \\
\hline Casalino and Barbarino [50] & 2010 & [ & & $\mathbf{\square}$ & \\
\hline Dieste and Gabard [53] & 2012 & & & $\mathbf{\square}$ & \\
\hline Kim and Haeri [54] & 2015 & & & $\mathbf{\square}$ & \\
\hline Miotto et al. [55] & 2017 & & & [ & \\
\hline Gea-Aguilera, Gill, and Zhang [56] & 2017 & & & $\mathbf{\square}$ & \\
\hline
\end{tabular}

was to improve and validate the SNGR model. In order to explore the limits to increase the understanding of broadband blade noise in the late 90s, Lockard and Morris [38] used a two dimensional Navier-Stokes code on the IBM SP2 to simulate the interaction of a vortical gust and NACA airfoils. The goal of the study was to study the influence of gust frequency and airfoil thickness on the noise generation. Ewert [46] introduced random particle-mesh (RPM) $\operatorname{method}_{-}^{6}$ which is capable of generating an artificial turbulent velocity field which reproduces a second-order two-point correlation tensor of homogeneous isotropic turbulence in two and three dimensions. Both of these stochastic methods, SNGR and RPM, derive from two main families of generating synthetic turbulence, the first of which was given by Kraichnan [58] and it is based on the idea that spectral components of solenoidal velocity fluctuations can be sampled in the wavenumber space from a prescribed mono-dimensional energy spectrum. The second family is based on the idea put forward by Careta, Gagues, and Sanch [59], according to whom turbulent fluctuations of prescribed two-point statistical properties can be obtained by filtering white noise.

\subsubsection{Lagrangian approach}

One of the first studies in broadband noise prediction using Lagrangian approach was conducted by Gennaretti, Luceri, and Morino [35] in which a unified, potential-flow, boundary-integral formulation was presented for studying velocity and pressure fields around rotors in hover and forward flight, thereby providing a tool for an integrated analysis of aerodynamics and aeroacoustics in linear as well as non-linear problems. Later, Guidati et al. [36] also used potential theory and implemented Boundary Element Method (BEM) in which a source term for inflow-turbulence noise is modelled by considering the spectral decomposition of a point

\footnotetext{
${ }^{6}$ The method is a further development of the technique used by Klein, Sadiki, and Janicka [57] to set up fluctuations at a LES inflow boundary.
} 
vortex which is convected along streamlines of the mean flow. Other significant studies $[39, \underline{43}$, 44] are conducted based on the same idea put forward by Gennaretti. A detailed discussion Lagrangian approach is given in sec. (3.1) , since the same approach is used in this thesis.

\subsubsection{Frequency domain and time domain comparison}

Acoustic radiation from a body immersed in a turbulent flow field, popularly known as leading-edge noise, is a widely studied topic in Computational Aeroacoustics (CAA). The early studies $[60,61,62]$ show the use of frequency domain analysis in which the incoming disturbance is modelled as a series of harmonic gusts by analysing the fields Fourier-transform. Studies based on this method have accounted for the effects of thickness, camber, and angle of attack, but were mainly used for relatively low frequencies. As the frequency increases, the spatial and temporal resolution requirements make these numerical methods more difficult to apply [63]. Another prevalent method in use is the time domain analysis $[\underline{64}, \underline{65}, \underline{66}$ which uses a set of discrete vortices to realise the turbulent inflow. However, these studies have not considered the statistical modelling of the inflow due to which the stochastic nature of the disturbance was missing. In more recent papers, Glegg [67] used a time domain method where the statistical nature of the disturbance was modelled using discrete vortices. Both methods, frequency domain and time-domain, have their advantages and limitations which are presented by Grace [68]. She concluded that the time domain analysis has an advantage over the frequency domain, primarily due to the ability to realise the turbulent inflow with the desired statistical properties.

Besides, using only an experimental method to address the effect of the interaction is also not a viable option because of its high cost.

\subsection{Motivation}

While individual aircrafts have become nearly $75 \%$ less noisy in the last few decades, with the ever-increasing air traffic, it seems we have to increase this percentage further as many European Union citizens are still in contact with the high noise level. The World Health Organisation (WHO) estimated that between 1 and 1.6 million healthy life years (DisabilityAdjusted Life Years) are lost annually because of environmental noise exposure, such as road traffic noise and aircraft noise, in European countries. According to their report, each year, around 1 million DALYS are lost due to sleep disturbance due to noise annoyance, heart disease, and cognitive impairment in children. A recent review study [69] suggested that risk for cardiovascular outcomes such as high blood pressure (hypertension), heart attack, and stroke increases by 7 to $17 \%$ for a $10 \mathrm{~dB}$ increase in aircraft noise exposure.

Besides the health issues, aircraft noise is one of the main reasons behind an adverse community reaction to the operation and expansion of airports. The Advisory Council for Aerospace Research in Europe (ACARE) has set the goal to reduce the noise emission of flying aircraft by $65 \%$ relative to the capabilities of typical new aircraft in 2000 . Therefore the acoustic signature is one of the main concerns for modern turbofan engines and the propulsion systems under development. This fact reflects a critical need for fast and reliable noise prediction techniques that can be used in the preliminary design stages by engine manufacturers to understand the 
sources and flow physics. Similarly, inspired by the rising interest in renewable energy for a sustainable future, the plans to create new wind farms generally face the opposition of the general public due to annoying noise levels. For high-speed trains, uniquely unconventional concepts like the magnetic levitation hover train projects, the relevance of aerodynamic noise is increased.

An important noise generation mechanism encountered in aeroengines is the interaction of incoming turbulence with the blades of the fan, concisely discussed in preceding sections. Due to considerable efforts in tonal noise reduction [70, 71], broadband noise reduction at the source remains a topic of significant interest for the industry and the research community in general. The methods which are available to analyse this behaviour range from analytical ones, which are fast but not precise enough to capture nonlinear effects, to numerical ones, which heavily depend on the computing resources [72].

More recently, nature-inspired designs are drawing the attention of many researchers. A study on humpback whale leading-edge tubercles highlighted the aerodynamic benefits [73]. Later on, it was found out that these wavy edges are an effective aeroacoustic treatment too. Clair et al. [74] experimentally and numerically investigated the effects of wavy leading-edges on the airfoil-turbulence interaction noise, and a broadband noise reduction of 3-4 $\mathrm{dB}$ was reported. Another design study, inspired by the silent flight of owls [75], has generated a great deal of recent research into the effectiveness of leading and trailing edge serrations. Regardless of the existence of these nature-inspired designs in nature for an extended period of time, due to an unavailability of a faster and efficient prediction methodology, it took a lot of time to bring these designs into practical applications.

A wide range of analytical models $[\underline{21}, \underline{19}]$ are available, which have the crucial benefit of being incredibly fast to produce noise predictions, and thus to understand the airfoil-turbulence interaction noise. These models, however, have restrictions that limit their application in the conceptual phase of design processes where the central objective is to help engineers and designers find the influence of design variables on the design of the machinery, quantitatively and qualitatively in the shortest possible time. In practical applications, there are many factors that can influence the accuracy of the analytical predictions such as the blade geometry, the turbulence statistics, the background mean flow distribution and the cascade effect. It is, of course, desirable to study the implications of these factors in real applications to aid the practical design process. This is undoubtedly the primary motivation for this thesis, which encourages the development of a low-fidelity numerical method capable of calculating the effect of geometric and flow-dependent variables on the overall blade design and allow its quick optimisation. Such a method depends on accurately modelling two fundamental processes: the realistic inflow turbulence (what exactly should be an input to the model), and the generation of noise by the turbulence interacting with the aerofoil structure (so-called leading-edge noise).

\subsection{Scope of the thesis}

The objectives of this thesis are:

i) To develop a time-domain numerical methods, based on the statistical properties on vortex particles, capable of predicting the acoustic radiation due to changes in geometric 
variables (airfoil type, shape, angle of attack, chord length) as well as flow field variables (turbulence intensity and length scale).

ii) To develop a fluid flow solver which can simulate unsteady/transient simulations, and predict time-dependent aerodynamic coefficients correctly.

iii) To generate inflow turbulence exhibiting the statistics of atmospheric turbulence for the flow solver in an inexpensive way.

iv) To investigate the application of discrete vortices to realise turbulence for aeroacoustic applications.

v) To predict the far-field noise from a realistic geometry in a realistic flow-field.

\subsection{Thesis structure}

i) Chapter 1: It presents a detailed literature survey on leading-edge noise and techniques used to calculate. A contrasting comparison between classical analytical studies and state-of-the-art techniques is stated. This is followed by the importance and objectives of the thesis.

ii) Chapter 2: A detailed distinction between Eulerian and Lagrangian way of looking at fluid is presented with the corresponding form of governing equations. The basics of vortex methods for incompressible and inviscid flow are addressed, which are then used to formulate the flow around an airfoil using stream function formulation. Later, the development of the fluid flow solver based on the Green's function solution of Laplace's equation is presented. The results from both steady and unsteady flow analyses are given at the end and are validated against the results from analytical formulations as well as from xfoil.

iii) Chapter 3: A statistical examination of velocity induced by a single vortex particle as well as multiple vortex particles at a generic location in the Cartesian coordinate system is presented which helps in understanding the effect of vortex parameters such as circulation strength and radius on the strength of induced velocity. The reconstruction of a fluctuating velocity signal using a set of vortex particles rests upon the ability to superimpose the disturbances associated with each particle, which is why the statistical study is carried out and presented in this chapter. The singularity problem associated with the point vortex is considered and is eliminated by using vortex particles with mollified kernels. Application of probability density functions to describe the nature of the variables, which characterise a vortex is addressed. Later, the generation of a realistic two-dimensional turbulent field using randomly distributed mollified vortices is presented.

iv) Chapter 3: Association between the vortex parameters (position vector, strength, and radius) and the attributes characterising turbulence (turbulence intensity and length scale) is given.

v) Chapter 4: Steady background flow around an airfoil using the panel method, developed in chapter 2 , is calculated. The analysis is then used to get the streamlines on which 
massless vortex particle is released well upstream the airfoil and allowed until well downstream the airfoil. This step is repeated for all relevant points from where a vortex can be introduced into the domain. The farfield sound pressure due to each and every vortex (strength, size) that started from a particular point upstream is computed and stored in a matrix, which is termed as the vortex-sound database.

vi) Chapter 4: Two separate vortex-sound databases for NACA0010 and NACA0012 airfoil are generated from which three isotropic homogeneous turbulent inflow signals (characterised by $1.19 \%, 4.9 \%, 13.2 \%$ turbulence intensity and $4.21 \mathrm{~mm}, 4.29 \mathrm{~mm}, 5.5$ $\mathrm{mm}$ integral length scale) are created. By introducing these three signals on the airfoil at $\alpha=0$, the sound pressure level was determined, which after $3 \mathrm{D}$ correction appeared to be in good agreement with the results obtained by solving the same case by Amiet's analytic formula. 



\title{
2
}

\section{Inviscid and Incompressible Flow}

\author{
Sparsh Sharma and Ennes Sarradj. "Fluctuating Inflow Condition for Time-Domain \\ Boundary Element Method for Airfoil-Turbulence Interaction Noise". In:ISUAAAT152018. \\ Oxford, UK, 2018
}

Chapter-1 introduces a phenomenon in which a body immersed in a turbulent flow experiences a fluctuating force over its surface, which results in noise radiation from the body. The direct approach to predict the noise radiation is computationally expensive, and thus not practised in the engineering design process. In the indirect approach, the unsteady aerodynamic effects arising due to turbulent flow are calculated separately using computational fluid dynamics and then used as input into the acoustic solver (based on for example Ffowcs-Williams and Hawkings (FW-H) equations). This chapter lays the mathematical foundation of the proposed technique based on vortex methods to estimate unsteady aerodynamic effects.

The differential equations that describe the motion of a Newtonian fluid are based on the principles of conservation of mass, momentum and energy and are known as the Navier-Stokes equations (NSE). The NSEs do not have a general analytic solution; there are only a few exceptional cases that can be solved analytically. The reason for not having solutions to these partial differential equations (PDE) is a complex nonlinearity that renders them. The more complex flow, the stronger the nonlinearity will be of the PDE. For practical problems, the solution of the NSEs is sought numerically. With increasing Reynolds number, Re, the computing requirements increase because of the finer mesh requirements to capture the smaller scale phenomena.

For incompressible flow, the fluid properties such as density and dynamic viscosity in the conservation equations are not affected by temperature. This assumption allows us to ignore the conservation equation for energy and concentrate only on the conservation equations for mass and momentum. This chapter addresses the development and validation of fluid-flow solver based on the Lagrangian approach. The solver is used to obtain the steady-state solution of flow around an airfoil, which allows the computation of streamlines in the domain of interest. 


\subsection{Description of Fluid Behaviour}

The two methods that are widely used to observe and analyse fluid flow are Lagrangian and Eulerian point of view. The distinction lies in the choice of point of view we wish to use to describe flow phenomena.

$$
\mathbf{u}_{\mathbf{v}_{n}} \forall n \in\{1,2, \ldots, n\}
$$

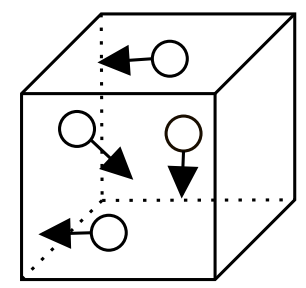

Lagrangian approach $\mathbf{u}$

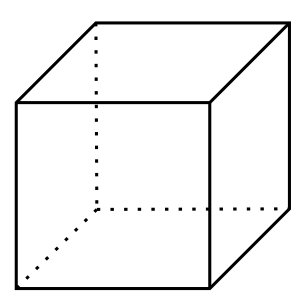

Eulerian approach

Figure 2.1: Lagrangian and Eulerian point of view approach to describe fluid behaviour

The Lagrangian description employs the particle point of view approach where individual fluid particles are tracked. It requires the evaluation of the motion of each fluid particle. In the Eulerian description, the observation point is fixed at a specific location in the flow field. The location of the observation point is kept constant, unlike in Lagrangian methods, and it is the change of the values of the dependent variables at the observation point that describes the flow field. Fig. (2.1) shows a schematic illustration of the treatment of the flow field in both approaches.

\subsection{Different Formulations for the Incompressible Navier- Stokes Equation}

The motion of an incompressible Newtonian fluid is governed by the following equations that express the conservation of mass and momentum of fluid in Eulerian and Lagrangian frames. The conservation of momentum and mass can be expressed as

velocity-pressure (primitive variable) formulation

$$
\begin{gathered}
\underbrace{\rho \frac{\partial \mathbf{u}}{\partial t}}_{\begin{array}{c}
\text { Rate of increase } \\
\text { of momentum }
\end{array}}+\underbrace{\rho \mathbf{u} \cdot \nabla \mathbf{u}}_{\begin{array}{c}
\text { Net flow rate of } \\
\text { momentum carried } \\
\text { by } \rho \mathbf{u}
\end{array}}=\underbrace{-\nabla p}_{\begin{array}{c}
\text { Net pressure } \\
\text { force }
\end{array}}+\underbrace{\mu \Delta \mathbf{u}}_{\begin{array}{c}
\text { Net viscous } \\
\text { force }
\end{array}}, \\
\nabla \cdot \mathbf{u}=0,
\end{gathered}
$$

where $\rho$ denotes the density, $\mathbf{u}$ denotes the velocity field, $p$ denotes the pressure, and $\mu$ denotes the dynamic viscosity of the fluid. Eq. $\underline{(2.1)}$ and (2.2) represent the velocity-pressure (primitive variable) formulation. In this formulation, there are three unknown velocity components and 
one unknown pressure to be solved in three-dimensions. A set of appropriate initial and boundary conditions are provided to above equations.

The second formulation is based on the concept of vorticity-streamfunction. It is advisable to concisely consider how does vorticity play an essential role in fluid dynamics analysis. In many cases $[76, \underline{77}, \underline{78}, \underline{79}, \underline{80}, \underline{81}, \underline{82}$ it is advantageous to analyse the dynamic events in a flow in terms of the evolution of the vorticity field. The vorticity field $(\omega)$ is related to the velocity field of flow as

$$
\omega=\nabla \times \mathbf{u}
$$

and is a solenoidal field $(\nabla \cdot \omega=\mathbf{0})$. The circulation $\Gamma$ of the vorticity field around a closed curve $l_{c}$, surrounding a surface $\mathrm{S}$ with a unit normal $\mathbf{n}$ is defined by

$$
\Gamma=\int_{l_{c}} \mathbf{u} \cdot d \mathbf{r}
$$

where $d \mathbf{r}$ denotes an element of the curve.

In two-dimensional flow, the vorticity vector has only one non-zero component which is perpendicular to the plane of the flow and thus is a scalar field $\omega$. By applying the curl operator on Eq. (2.1), the vorticity equation is obtained as

vorticity-velocity formulation

$$
\underbrace{\rho \frac{\partial \omega}{\partial t}}_{\begin{array}{c}
\text { Rate of increase } \\
\text { of vorticity }
\end{array}}+\underbrace{\rho \mathbf{u} \cdot \nabla \omega}_{\begin{array}{c}
\text { Net flow rate } \\
\text { of vorticity }
\end{array}}=\underbrace{\rho \omega \cdot \nabla \mathbf{u}}_{\begin{array}{c}
\text { Vortex } \\
\text { stretching }
\end{array}}+\underbrace{\mu \Delta \omega}_{\begin{array}{c}
\text { Viscous } \\
\text { diffusion }
\end{array}} .
$$

Eq. (2.5) does not include the pressure variable because for a homogeneous incompressible fluid, $\nabla \times \nabla p=0$. This decoupling of the velocity and the pressure terms is one of the main advantages of this formulation.

\subsection{Incompressible Vortical flow}

For incompressible flow, $\rho=\rho_{\infty}=1$, and the kinematic viscosity $\nu=\mu$ The vortex methods are based on the Lagrangian formulation of these equations, and in two-dimensions, the vorticity equation reduces to the scalar vorticity equation [83]

$$
\frac{\mathrm{D} \omega}{\mathrm{D} t}=\nu \Delta \omega
$$

Recalling that for inviscid flow $(\nu=0)$ this equation implies conservation of vorticity along the particle trajectories $X(\xi, t)_{-}^{1}$ :

$$
\omega(X(\xi, t), t)=\omega_{0}(\alpha), \quad \xi \in \mathbb{R}^{2} .
$$

\footnotetext{
${ }^{1}$ interested readers can refer to literature 'Vorticity and Incompressible flow' by Andrew Majda
} 
Eq. (2.7) distinguishes 2D from 3D inviscid flow, general 3D flow have $\omega \cdot \nabla \mathbf{u} \neq 0$, and the vorticity stretches according to the formula

$$
\omega(X(\xi, t), t)=\nabla_{\xi} X(\xi, t) \omega_{0}(\alpha), \quad \xi \in \mathbb{R}^{3} .
$$

In this thesis, fluid is being mainly analysed in two dimensions. It is known to the author that fluid flow in real world occurs in three space dimensions. For 2-D flow the velocity field is $\mathbf{u}=\left(u_{1}, u_{2}, 0\right)^{t}$, the vorticity $\omega=\left(0,0, \omega_{3}\right)^{t}$ is orthogonal to $\mathbf{u}$, and the vorticity stretching term vanishes. Thus the resulting set of equations, Euler equations in two-dimensions, are

$$
\begin{gathered}
\frac{\partial \omega}{\partial t}+\nabla \cdot(\mathbf{u} \omega)=0 \\
\omega(\cdot, 0)=\omega_{0} \\
\nabla \cdot \mathbf{u}=0 \\
\nabla \times \mathbf{u}=\omega \\
|\mathbf{u}| \stackrel{\infty}{\rightarrow} \mathbf{u}_{\infty}
\end{gathered}
$$

A classical way to relate the velocity and vorticity [Eqs. (2.9)-(2.13)] is through an integral representation. If $G$ denotes the Green's function for the Laplacian operator in two-dimensions, and $\mathbf{K}$ denotes the rotational counterpart, then for $\mathbf{x}=\left(x_{1}, x_{2}\right)$, we have:

$$
G(\mathbf{x})=-\frac{1}{2 \pi} \log (|\mathbf{x}|) ; \quad \mathbf{K}(\mathbf{x})=\left(2 \pi|\mathbf{x}|^{2}\right)^{-1}\left(-x_{2}, x_{1}\right)
$$

and the Biot-Savart law reads

$$
\mathbf{u}=\mathbf{U}+\mathbf{K} \star \omega .
$$

The term omega in Eq. (2.15) approximates the initial circulation concentrated on a single point or a particle in the computational domain as

$$
\omega_{0}=\sum_{N} \alpha_{N} \delta\left(\mathbf{x}-\mathbf{x}^{\prime}\right),
$$

where the value of $\alpha$ is an estimate of the initial circulation around $\mathrm{N} t h$ particle at $\mathbf{x}^{\prime}$. The kernel $\mathbf{K}$ is associated with a singularity which while implementing directly with the vorticity field $\omega$ can lead to enormous values when two particles come close to each other. (This peculiarity is discussed in sec. $(\underline{3.6 .1})$, with its elimination in sec. $(\underline{3.6 .3})$ )

The development of vortex methods, based on the fundamental equations mentioned above, is given in the next section. 


\subsubsection{Vortex Methods}

The vorticity carried by the fluid elements is conserved in inviscid flow and simulating the flow amounts to the computation of the velocity field. In inviscid flow, the velocity field is split into two parts, the Rotational Velocity Component and Potential Velocity Component. Rotational velocity refers to the velocity field produced due to the vorticity present in the flow field, whereas the potential velocity component is used to implement the boundary conditions and to ensure the compatibility of the velocity and the vorticity field in the presence of boundaries. This velocity decomposition is famously known as Helmholtz decomposition.

\subsection{2 $\Psi-$ formulation}

The basic idea behind vortex methods is to approximate the continuous vorticity field, $\omega$, by a set of discrete particles. The kinematics of these particles, governed by the Hamiltonian (discussed in the next section), can be enforced using Helmholtz decomposition or the vorticityvelocity formulations [83]. The velocity and the vorticity field is described with the aid of a single vector streamfunction. For an incompressible flow field, a single vector streamfunction $\boldsymbol{\Psi}$ such that the velocity $\mathbf{u}$ is its curl:

$$
\mathbf{u}=\nabla \times \mathbf{\Psi}
$$

This single vector streamfunction $\boldsymbol{\Psi}$ unifies the streamfunctions for each constituent of the flow field, $\Psi_{\omega}$, the vorticity field in the field, $\Psi_{\gamma}$, the vortex sheet distribution on the boundary (body), and $\Psi_{\infty}$, the streamfunction corresponding to the velocity field at infinity.

$$
\boldsymbol{\Psi}=\boldsymbol{\Psi}_{\omega}+\boldsymbol{\Psi}_{\gamma}+\boldsymbol{\Psi}_{\infty}
$$

Let's recall the vorticity-velocity formulation of the Euler equations expresses the conservation of vorticity along fluid element paths. The vorticity field and the streamfunction are related as

$$
\omega=\nabla \times \mathbf{u}=\nabla \times \nabla \times \boldsymbol{\Psi}=\nabla(\nabla \cdot \boldsymbol{\Psi})-\nabla^{2} \boldsymbol{\Psi} .
$$

Since the vector streamfunction is solenoidal $(\nabla \cdot \boldsymbol{\Psi}=0)$, a Poisson equation is obtained for the streamfunction and the vorticity field:

$$
\nabla^{2} \Psi=-\omega
$$

Applying the Poincaré identity to each component of the streamfunction yields

$$
\begin{aligned}
\boldsymbol{\Psi}(\mathbf{x})= & \underbrace{\int_{\omega} \omega\left(\mathbf{x}^{\prime}\right) G\left(\mathbf{x}-\mathbf{x}^{\prime}\right) d V^{l}\left(\mathbf{x}^{\prime}\right)}_{\Psi_{i}^{l}} \\
& +\underbrace{\int_{S}\left(\frac{\partial\left(\mathbf{\Psi}_{\mathbf{i}}-\mathbf{\Psi}_{\mathbf{e}}\right)}{\partial \mathbf{n}}\left(\mathbf{x}^{\prime}\right) G\left(\mathbf{x}-\mathbf{x}^{\prime}\right)\right)-\left(\left(\mathbf{\Psi}_{\mathbf{i}}-\mathbf{\Psi}_{\mathbf{e}}\right)\left(\mathbf{x}^{\prime}\right) \frac{\partial G}{\partial \mathbf{n}}\left(\mathbf{x}-\mathbf{x}^{\prime}\right) d S\left(\mathbf{x}^{\prime}\right)\right)}_{\Psi_{\gamma}} \\
& +\Psi_{\infty} .
\end{aligned}
$$


The expression given in Eq. (2.21) relates the vorticity to the kinematics of the vortex particles in the flow field. The above equations can be expressed in terms of singularity distributions on the boundary of the domain. The source distribution is defined as

$$
\boldsymbol{\sigma}_{s}=\boldsymbol{\Psi}_{o}-\boldsymbol{\Psi}_{i}
$$

where the subscript $o$ and $i$ represent the volume outside the body and the volume inside the body, respectively in the domain. The vortex sheet is defined as

$$
\gamma_{s}=\frac{\partial \boldsymbol{\Psi}_{o}}{\partial \mathbf{n}}-\frac{\partial \boldsymbol{\Psi}_{i}}{\partial \mathbf{n}}
$$

The discretised vorticity field is expressed as the sum of the vorticities of all the vortex particles, in which the initial circulation is concentrated, in the following way:

$$
\omega_{0}=\sum_{p} \Gamma_{p} \delta\left(\mathbf{x}-\mathbf{x}^{\prime}(t)\right)
$$

Vorticity is constantly created at the fluid-solid interface, i.e. the surface of the immersed body, as a consequence of continuously satisfying the surface velocity boundary conditions. No-slip and no-penetration boundary condition are enforced at the centre point of all the boundary elements (panels) [84], which are discretised straight line elements of finite length, $\mathrm{N}$ in numbers over the body surface. The domain is discretised into computational nodes, interpreted as either of sources, doublets, vortex particles or the combination of two or all [85]. The surface (sheet) circulation computed by the BEM is then released into the flow at each time step as new circulation-carrying particles (vortices).

Using Eq. (2.17), the generalised velocity terms are written as

$$
\begin{gathered}
\mathbf{u}_{\omega}=\nabla \times \int_{V_{i}} \mathbf{G}\left(\mathbf{x}-\mathbf{x}^{\prime}\right) \omega\left(\mathbf{x}^{\prime}\right) d V\left(\mathbf{x}^{\prime}\right)=\int_{V_{i}} \mathbf{K}(\mathbf{x}-\mathbf{y}) \omega(\mathbf{y}) d \mathbf{y}=\mathbf{K} \star \omega \\
\mathbf{u}_{\gamma}=\nabla \times \int_{S}\left[\gamma\left(\mathbf{x}^{\prime}\right) G\left(\mathbf{x}-\mathbf{x}^{\prime}\right)-\sigma_{s}\left(\mathbf{x}^{\prime}\right) \frac{\partial G}{\partial \mathbf{n}}\left(\mathbf{x}-\mathbf{x}^{\prime}\right)\right] d S\left(\mathbf{x}^{\prime}\right),
\end{gathered}
$$

where $\star$ and $\mathbf{K}$ denotes convolution and Biot-Savart kernel, respectively.

\subsubsection{Vortex particle kinematics}

Consider two-dimensional flow, where the incompressibility constraint $\nabla \cdot \mathbf{u}=0$ is satisfied by taking $u=-\partial \psi / \partial y, v=\partial \psi / \partial x$. The stream function $\psi(\mathbf{x}, t)$ (which is a conserved quantity [86]) plays the role of the Hamiltonian for the coordinates $(x, y)$ of the vortex particle whose dynamics is given by

$$
\frac{d x_{i}}{d t}=\frac{d \psi}{d y_{i}}, \quad \frac{d y_{i}}{d t}=-\frac{d \psi}{d x_{i}}
$$

and can be written as

$$
\psi\left(\mathbf{x}_{1}, \ldots, \mathbf{x}_{\mathbf{N}}\right)=-\sum_{i=1}^{N} \sum_{j=i+1}^{N} \Gamma_{i} \Gamma_{j} G\left(\mathbf{x}_{i}, \mathbf{x}_{j}\right)-\frac{1}{2} \sum_{i=1}^{N} \Gamma_{i}^{2} g\left(\mathbf{x}_{i}, \mathbf{x}_{i}\right),
$$


where $\Gamma$ represents the circulation. The first term on the right-hand side is the Green's function of the first kind for the domain, defined by

$$
\mathcal{L} G\left(\mathbf{x}, \mathbf{x}^{\prime}\right)=\delta\left(\mathbf{x}, \mathbf{x}^{\prime}\right) .
$$

$\mathcal{L}$, the linear elliptic operator is taken to be the Laplacian ( $\mathcal{L} \equiv \Delta$, for most of the formulations), and the result is the point vortex dynamical system of the $2 \mathrm{D}$ Euler equations. The second term, $g\left(\mathbf{x}, \mathbf{x}^{\prime}\right)$, is the residual Green's function defined by

$$
g\left(\mathbf{x}, \mathbf{x}^{\prime}\right)=G\left(\mathbf{x}, \mathbf{x}^{\prime}\right)-G_{0}\left(\mathbf{x}, \mathbf{x}^{\prime}\right),
$$

where $G_{0}$ corresponds to the free-space Green's function. Numerical solution of the system Eq. $(\underline{(2.27)})$ has understandably focused on the 2D Euler system, for which

$$
\mathcal{L} \equiv \Delta, \quad G_{0}\left(\mathbf{x}, \mathbf{x}^{\prime}\right)=\frac{1}{2 \pi} \log \left(\left|\mathbf{x}-\mathbf{x}^{\prime}\right|\right) .
$$

The Green's function formulation for $\mathcal{L}$, the linear elliptic operator, Eq.(2.31) is an important result which is used in the next section.

\subsection{Background flow: Potential flow}

From here, the topic of discussion is moving to explain the stages of development of the flow solver. This solver is used to calculate the pathlines for the convection of the vortices, which are statistically optimised to posses desired statistics. Hence, an important and the first section of the indirect methodology to predict the airfoil-turbulence interaction noise. This section demonstrates explicitly the assumptions that make potential flow a potential flow and also introduces the solution to Laplace's equation by employing the Green's function formulation.

\subsubsection{Irrotational flow}

The vorticity in high Reynolds number flow fields is confined to the boundary layer and the wake regions where the effects of viscosity are dominant; therefore, it is appropriate to consider that the flow in the area outside these confined areas is irrotational as well as inviscid. In such flow, the velocity vector $\mathbf{u}$ can be calculated as the gradient of a potential $\phi$ as

$$
\mathbf{u}=\nabla \phi(x, y, z)
$$

The function $\phi$ is a spatial function.

In Cartesian coordinates, the velocity components are given by

$$
u=\frac{\partial \phi}{\partial x}, \quad v=\frac{\partial \phi}{\partial y}, \quad w=\frac{\partial \phi}{\partial z}
$$

An arbitrary solid body immersed in an incompressible and irrotational fluid, defining a velocity potential $\phi$ and combining it with the continuity equation gives rise to a second-order partial 
differential equation. By substituting Eq. (2.32) into Eq. (2.11), the following equation for the velocity potential is obtained:

$$
\nabla^{2} \phi=0
$$

For 2D flow, considerable computational time saving is derived as the vorticity has a single component (in out-of-flow plane). This means that for a flow field described in $x y$-plane, the nonzero component of vorticity is in the $z$-direction and will still be considered as vector potential. The vector potential for $2 \mathrm{D}$ flow is the stream function $(\psi)$ and has the only nonzero component in the $z$-direction. Thus, for 2D irrotational flow, Eq. $\underline{2.34}$ can also be written as

$$
\nabla^{2} \psi=0_{-}^{2}
$$

The above equation, commonly known as Laplace's equation, belongs to the elliptic partial differential equation class, and an elliptic PDE have characteristics which occur in a pair, each a complex conjugate of other. Since this class of problems have the highest derivative given by $\nabla^{2 n}$, it requires $\mathrm{n}$ boundary conditions to solve it.

\subsubsection{Boundary conditions and uniqueness of the solution}

Laplace's equation, a partial differential equation for the velocity for an inviscid, incompressible, an irrotational flow, is an elliptic differential equation that results in a boundary-value problem. For a problem involving solid surfaces, the boundary conditions need to be specified on all solid surfaces and at infinity. This thesis deals with a problem that involves an aerodynamic problem involving solid surfaces submerged in a fluid, which require the boundary conditions on all solid-fluid surfaces and infinity. Typical boundary conditions for such problems require that on stationary solid surface boundaries both the normal and tangential velocity components reduce to zero as

$$
\begin{aligned}
& u_{n}=0 \quad \text { (on solid surface) } \\
& u_{t}=0 \quad \text { (on solid surface) }
\end{aligned}
$$

As the viscosity of the fluid is neglected here $(\mu=0)$, the no-slip-condition boundary condition cannot be applied to the solid-fluid boundary, resulting of which only Eq. $\underline{(2.36)}$ will be used. This boundary condition is enforced on the normal derivative of the potential and is called as Neumann boundary condition. In the theory of linear partial differential equations, a well-posed problem consists of a linear partial differential equation subject to certain boundary conditions such that the solution is unique [7]. In addition, there is one more condition which states that the disturbance created by the solid body should become zero as the position of influence tends to infinity,$$
\nabla \phi \rightarrow U \quad \text { for } \vec{x} \rightarrow \infty
$$

${ }^{2}$ Poisson's equation for rotational flow: $\nabla^{2} \psi=\omega$
} 


\subsubsection{Solution}

The Numerical Solutions of Laplace's equation are divided into two classes. The first one is the grid-based (e.g., finite differences, finite volumes) in which the Laplacian operator is discretised over the entire domain, resulting in a group of linear equations for the unknown values of potential. For relatively simpler geometries the matrices involved are banded, and the equations can be solved easily with certain matrix inversion operations. The approach requires the discretisation of the whole domain, which is a costly means in itself. For complex geometries, the resulting set of a linear system of equations does not always have an uncomplicated structure which can make the computations more expensive.

The second method to obtain the solution of Laplace's equations is by employing the equivalent Green's function formulation ${ }^{3}$. This is done by enforcing the boundary conditions which results in a set of integral equations for an unknown distribution of discontinuities at the boundary of the domain. These equations are then discretised resulting in a set of algebraic equations for the unknown singularity strength at the domain boundary. This method allows an inexpensive way to deal with complex geometries since only the boundary of the domain needs to be discretised.

The integral equation technique to solve Laplace's equation for different geometries exploits the linearity of the equation. The three widely used integral solutions are $i$ ) linear superposition of elementary solutions of Laplace's equation (such as sources/sinks), ii) the method of images in which the boundary conditions are enforced by considering an odd or even extension (image) of the infinite-domain Green's function across the boundary, and iii) the method of singularity distribution in which the solution of Laplace's equation is expressed in terms of continuous superposition of its Green's functions with weighting strength, the discontinuities of the potential, and its derivatives at the boundary of the domain. The first two techniques can be viewed as particular cases of the last one.

The present work utilises the method of singularity distributions on the boundary of the domain, which is based on the Poincaré identity, which relates $\phi$ to its discontinuities at the boundary of the domain. For an incompressible flow with potential $\phi$, the Poincaré identity gives

$$
\phi(x)=\int_{S}[\underbrace{\left[\mathbf{n}\left(\mathbf{x}^{\prime}\right) \cdot \nabla\left(\phi_{i}-\phi_{e}\right)\left(\mathbf{x}^{\prime}\right) G\left(\mathbf{x}-\mathbf{x}^{\prime}\right)\right.}_{\text {single layer potential }}-\underbrace{\left(\phi_{i}-\phi_{e}\right)\left(\mathbf{x}^{\prime}\right) \mathbf{n}\left(\mathbf{x}^{\prime}\right) \cdot \nabla G\left(\mathbf{x}-\mathbf{x}^{\prime}\right)}_{\text {double layer potential }}] d S\left(\mathbf{x}^{\prime}\right),
$$

where

$$
G\left(\mathbf{x}-\mathbf{x}^{\prime}\right)=-\frac{1}{2 \pi} \log \left(\left|\mathbf{x}-\mathbf{x}^{\prime}\right|\right)
$$

The difference between the external and internal potentials can be defined as

$$
-\mu_{d}=\phi_{i}-\phi_{e},
$$

\footnotetext{
${ }^{3}$ Refer to Appendix A for Green's Functions and Solutions of Laplace's Equation
} 
and is called doublet or double-layer potentials $\left(\mu_{d}\right)$, whereas, the difference between the normal derivative of the external and internal potentials is called source or single-layer potentials $\left(\sigma_{s}\right)$ and defined as

$$
-\sigma_{s}=\frac{\partial \phi_{e}}{\partial \mathbf{n}}-\frac{\partial \phi_{i}}{\partial \mathbf{n}} .
$$

The strength of the distribution of doublets and sources on the surface must be determined to find the velocity potential in the domain. Eq. (2.39) does not specify a unique combination of source and doublets for a particular problem; instead, a choice must be made in this regard, which generally is based on the physics of the problem.

In this thesis, the solution of $\nabla^{2} \phi$ is obtained by distributing elementary solutions ${ }^{4}$ (source/sink, doublets, vortex) on the boundary of the domain. These elementary solutions fulfil the boundary condition of Eq. (2.38). Then, these singularity elements are distributed over the surface of the boundary of the domain and integrated to obtain the solution because the governing equation is elliptic in nature where a disturbance in the interior of the solution affects the solution everywhere else. The disturbance signals travel in all directions. Before applying this methodology to find the potential or streamfunction and thus the velocity field, the features of these singularity solutions and the problem to be solved using them are analysed in the next section.

\subsubsection{Source/Sink}

A source is imagined as a point from which the fluid is flowing out, uniformly. Thus, all the streamlines radiate from a single point as straight lines, and the radial velocity decreases with the distance from the source point. For a two-dimensional case, it is convenient to use the polar coordinate system, $(r, \theta)$, where the velocity components (radial and tangential) are given as

$$
u_{r}(r, \theta)=\frac{\sigma_{s}}{2 \pi r}, \quad u_{\theta}(r, \theta)=0
$$

where $\sigma_{s}$ denotes the source strength which represents the flux introduced by the source. Integrating above equations yield velocity potential as

$$
\phi=\frac{\sigma_{s}}{2 \pi} \log r+\text { constant. }
$$

The constant can be set to zero by assuming the point source is placed at the origin. The streamfunction can be obtained by using its relationship with the velocity components

$$
\frac{1}{r} \frac{\partial \psi}{\partial \theta}=u_{r}, \quad-\frac{\partial \psi}{\partial r}=u_{\theta}
$$

which integrates to

$$
\psi=\frac{\sigma}{2 \pi} \theta+\text { constant }
$$

In source flow, strength $\sigma_{s}$ is positive. A source with a negative strength is called a sink in which instead of radiating from a single point, the straight streamlines are now converging

\footnotetext{
${ }^{4}$ also known as singular solutions because at $\mathrm{x}-\mathrm{x}$ ' $=0$, the velocity becomes singular
} 


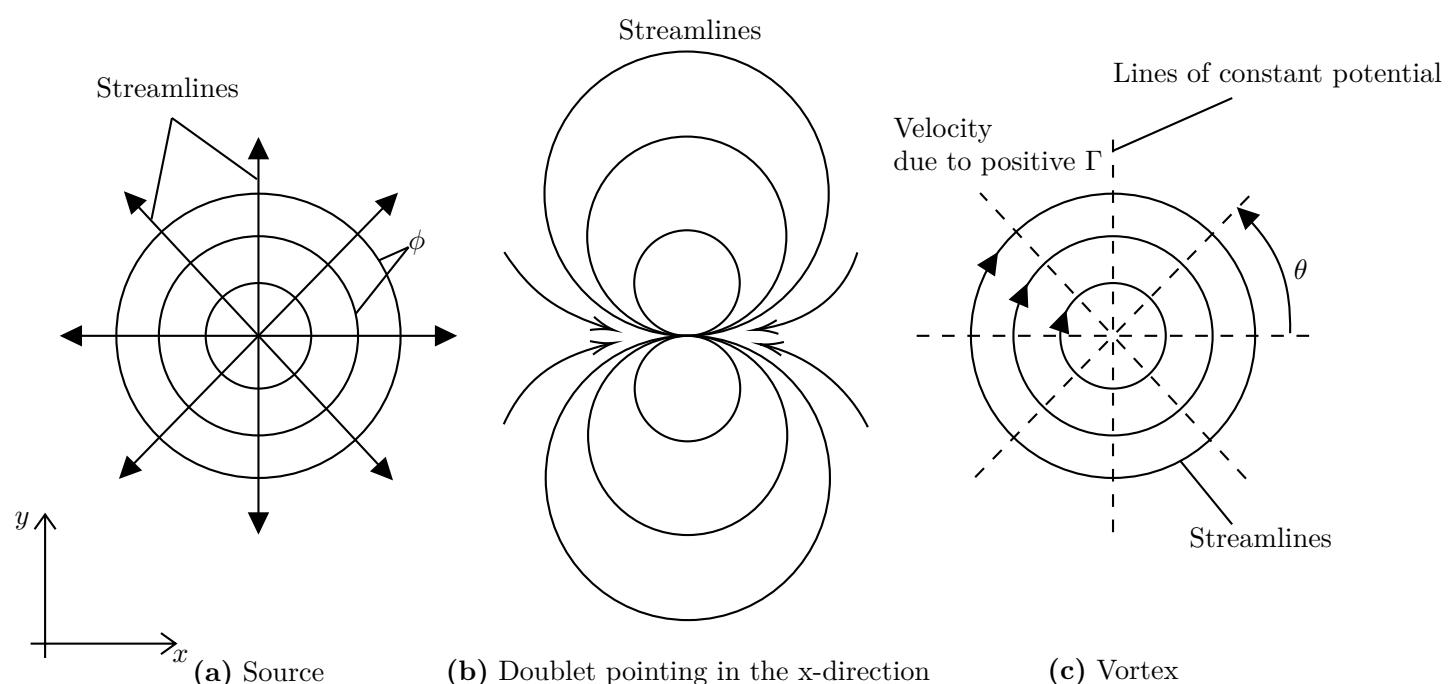

Figure 2.2: (a) Streamlines due to source element at the origin. (b) Streamlines due to a doublet at the origin. (c) Streamlines and equipotential lines for a vortex at the origin.

to a single point. The velocity field corresponding to a sink looks similar to that of a source, except for the direction of the flow.

\subsubsection{Doublet}

A doublet is obtained when a point source and a point sink approach each other with the separation distance between the approaches zero, with their separation distance multiplied by their strength such that the product remains constant $\left(\mu_{d}\right)$.

Doublet's potential function is given as

$$
\phi=-\frac{\mu_{d}}{2 \pi} \frac{\cos \theta}{r},
$$

and streamfunction as

$$
\psi=\frac{\mu_{d}}{2 \pi} \frac{\sin \theta}{r} .
$$

The tangential and radial velocities can be easily derived using Eq. (2.45).

\subsubsection{Vortex}

For an irrotational vortex, the circulation is zero along any closed contour that does not enclose the vortex axis; and has a fixed value, $\Gamma$, for any contour that does enclose the axis once. The tangential component of the particle velocity is then

$$
u_{\theta}(r, \theta)=\frac{\Gamma}{2 \pi r}
$$

while the radial velocity is zero,

$$
u_{r}(r, \theta)=0 .
$$




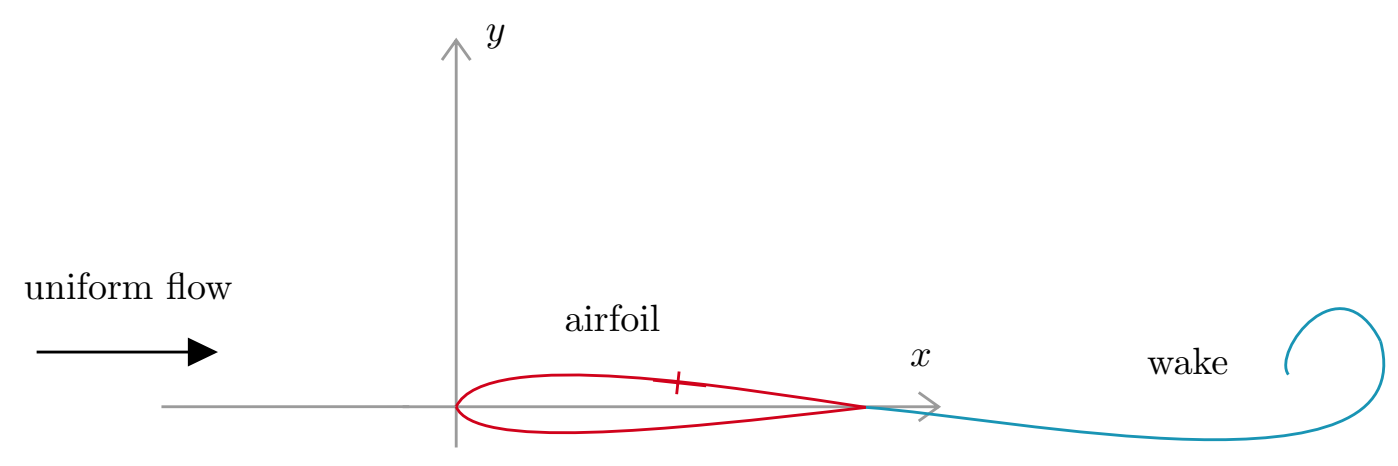

Figure 2.3: Lagrangian and Eulerian point of view approach to describe fluid behaviour

The streamfunction and potential are obtained by integrating the velocity components:

$$
\psi=\frac{\sigma_{s}}{2 \pi} \ln r, \quad \phi=-\frac{\sigma_{s}}{2 \pi} \theta,
$$

respectively. Fig. (2.2) shows the elementary solutions to Laplace's equation governing incompressible and irrotational fluid flow.

\subsection{Formulation of the problem: Unsteady flow over an airfoil}

Consider a two-dimensional airfoil, at rest, at the origin of an $x y$-coordinate system, where the chord line originating from the leading edge towards the trailing edge coincides with the $x$-axis as shown in Fig. (2.3). The airfoil is subjected to an incoming disturbance (discussed in the next chapter), making the problem a time-dependent where the aerodynamic coefficients fluctuate with time. The incoming disturbance causes airfoil to continuously shed vortices at the trailing edge, which distinguishes unsteady flow from steady flow. In this section, the flow field around the airfoil is formulated without any incoming disturbance.

To find the velocity potential in the computational domain, two main things to ponder:

- the strength of the distribution of the singularities on the surface must be determined,

- the specification of a unique combination of singularities for the problem of airfoil subjected to a uniform/nonuniform flow.

One of the first practical methods was developed by Hess and Smith [84] for steady flow problems. The approach was extended to unsteady flow problems by Basu and Hancock [76] and Teng [87]. The method is based on the distribution of sources and vortices on the surface of the geometry.

\subsubsection{Flow model}

Consider an airfoil at rest in an onset flow of velocity $U$. It is assumed that the airfoil is at an angle of attack, $\alpha$ (the angle between its chord line and the onset velocity). The geometry of the airfoil is then discretised into a finite number $(N)$ of short straight-line elements called 
panels, defined by $(N+1)\left(x_{j}, y_{j}\right)$ pairs called boundary points. The attributes of a panel are: its starting point $\left(x_{i}, y_{i}\right)$, endpoint $\left(x_{i+1}, y_{i+1}\right)$ and mid-point $\left(\bar{x}_{i}, \bar{y}_{i}\right)$, its orientation $\left(\theta_{i}\right)$ (inclination to the $x$-axis) and length $l_{i}$, and tangential unit vector $\hat{t}_{i}$ and normal unit vector $\hat{n}_{i}$ as shown in Fig (2.4a). It is common to input the $(x, y)$ coordinates starting at the lower surface trailing edge, proceeding clockwise around the airfoil, and ending back at the upper surface trailing edge. Hence, the pair $\left(x_{1}, y_{1}\right)$ and $\left(x_{N+1}, y_{N+1}\right)$ are identical and represent the trailing edge point. The element between $\left(x_{i}, y_{i}\right)$ and $\left(x_{i+1}, y_{i+1}\right)$ is referred to as the $i$-th panel, and the mid-points of the panels as collocation points. As mentioned in the preceding section, each of these panels is characterised by a constant source element and vorticity should be constant over all panels. Thus the velocity potential for the problem is given by

$$
\phi=U(\cos \alpha \hat{i}+\sin \alpha \hat{j})+\sum_{j=1}^{N} \int_{\text {panel } j}\left[\frac{\sigma_{j}(s)}{2 \pi} \ln \left(\mathbf{x}^{\prime}\right)_{j}-\frac{\gamma}{2 \pi} \theta_{j}\right] d s,
$$

where it can be seen that $\phi$ can be calculated by describing $(N+1)$ unknowns: $\sigma_{j}(j=$ $1,2, \ldots, N)$ and $\gamma$ using a numerical technique by satisfying the flow tangency at $N$ collocation points and satisfying the Kutta-condition.

The overall flow field, represented by $\phi$, is then used to calculate the velocity vector $\vec{V}$ at any point in the computational region using the definition given in Eq. (2.34).

\subsubsection{Discretisation into panels}

Discretising a thick airfoil needs dense panelling near the leading and trailing edges, hence dividing the airfoil perimeter into equal parts is not considered. Preferably, the cosine method is employed for dividing the chord into panels with higher density near the edges, as shown in Fig. (2.4b). For example, if five panels are required to discretise the geometry, then the semicircle encircling the upper curve is divided by this number resulting $\Delta \beta=\pi / 5$, which allows the calculation of corresponding abscissa, $x$, values using the cosine spacing formula $\left(x=\frac{c}{2}(1-\cos \beta)\right.$.

\subsubsection{Flow tangency boundary condition}

The flow tangency condition is applied at the panel mid-point as

$$
\vec{V} \cdot \hat{n}_{i} \equiv 0
$$

where $\vec{V}$ is velocity at the panel mid-point, and $\hat{n}$ is the normal unit vector. Enforcing the flow-tangency condition on each collocation point approximately makes the body geometry correspond to a dividing streamline.

\subsubsection{The Kutta condition}

The Kutta-condition states that the pressure below and above the airfoil trailing edge must be equal so that the flow does not bend around it and instead leaves the airfoil tangentially. The 


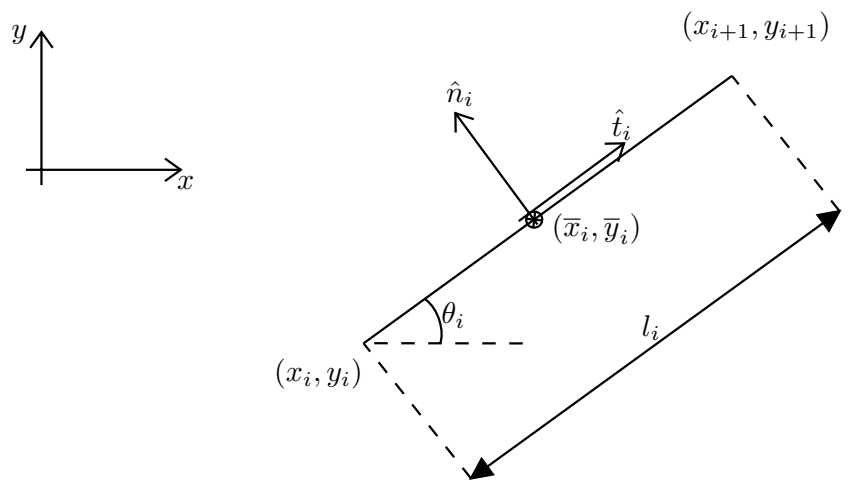

$(a)$

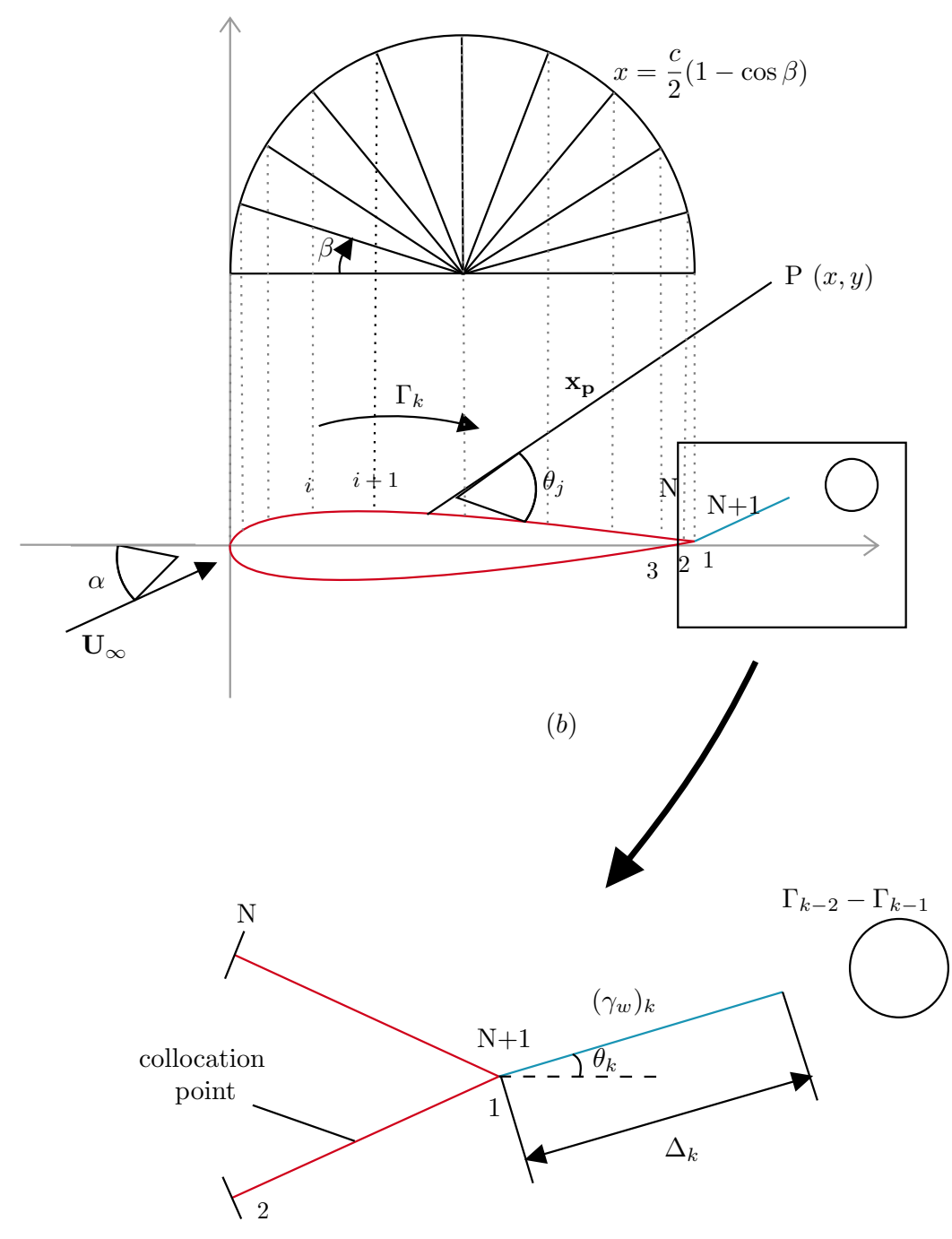

$(c)$

Figure 2.4: Panel method applied to an airfoil in unsteady flow conditions 
rear stagnation point must be exactly at the trailing edge. At the trailing edge of the airfoil, the Kutta condition can be written as

$$
\left(\vec{V} \cdot \hat{t}_{i}\right)_{i=1}=-\left(\vec{V} \cdot \hat{t}_{i}\right)_{i=N}
$$

where $\hat{t}$ is the tangential unit vector. There is a negative sign on the right-hand side of Eq. (2.54) because the tangent directions are opposite for $i$-th and $N$-th panel.

\subsection{Numerical method: linear system}

In this section, a linear system of equations is constructed using the boundary conditions given in Eqs. $(\underline{2.53)}$ and $(\underline{2.54})$. It is convenient to write the velocity $\vec{V}$ in terms of its velocity components $\left(u_{i}\right)$ and $\left(v_{i}\right)$ by

$$
\begin{gathered}
u_{i}=U \cos \alpha+\sum_{j=1}^{N} \sigma_{j} u_{i j}^{(s)}+\gamma \sum_{j=1}^{N} u_{i j}^{(v)}, \\
v_{i}=U \sin \alpha+\sum_{j=1}^{N} \sigma_{j} v_{i j}^{(s)}+\gamma \sum_{j=1}^{N} v_{i j}^{(v)},
\end{gathered}
$$

which are then used to derive the tangential and normal velocity components to enforce the boundary conditions as

$$
\begin{aligned}
& \vec{V} \cdot \hat{n}_{i}=\left(V^{n}\right)_{i} \\
& \quad=U \sin \left(\alpha-\theta_{i}\right)+\sum_{j=1, j \neq i}^{N} A_{i j}^{n} \sigma_{j}+\gamma \sum_{j=1, j \neq i}^{N} B_{i j}^{n}, \quad \text { (flow tangency) } \\
& \vec{V} \cdot \hat{t}_{i}=\left(V^{t}\right)_{i} \quad=U \cos \left(\alpha-\theta_{i}\right)+\sum_{j=1, j \neq i}^{N} A_{i j}^{t} \sigma_{j}+\gamma \sum_{j=1, j \neq i}^{N} B_{i j}^{t}, \quad \text { (Kutta condition) }
\end{aligned}
$$

In terms of unknowns, $\sigma_{j}(j=1,2, \ldots, N)$ and $\gamma$, the boundary conditions for a system of algebraic equations can be written in the following form,

$$
A \vec{x}=\vec{b}
$$


where $A$ is a square matrix of order $(N+1)$, that is

$$
\left|\begin{array}{ccccccc}
a_{11} & a_{12} & \ldots & a_{1 j} & \ldots & a_{1 N} & a_{1, N+1} \\
a_{21} & a_{22} & \ldots & a_{2 j} & \ldots & a_{2 N} & a_{2, N+1} \\
\vdots & \vdots & \vdots & \vdots & \vdots & \vdots & \vdots \\
a_{i 1} & a_{i 2} & \ldots & a_{i j} & \ldots & a_{i N} & a_{i, N+1} \\
\vdots & \vdots & \vdots & \vdots & \vdots & \vdots & \vdots \\
a_{N 1} & a_{N 2} & \ldots & a_{N j} & \ldots & a_{N N} & a_{N, N+1} \\
a_{N+1,1} & a_{N+1,2} & \ldots & a_{N+1, j} & \ldots & a_{N+1, N} & a_{N+1, N+1}
\end{array}\right|
$$

where $\vec{x}=\left(\sigma_{1}, \ldots, \sigma_{i}, \ldots, \sigma_{N}, \gamma\right)^{T 5}$ and $\vec{b}=\left(b_{1}, \ldots, b_{i}, \ldots, b_{N}, b_{N+1}\right)^{T}$. With all the elements of $a_{i j}$ and $b_{i j}$ determined, the solution of Eq. (2.59) is found out using lower-upper (LU) decomposition with partial pivoting and row interchanges $[\underline{88}, \underline{89}$.

\subsubsection{Computation of Pressure and Loads}

The pressure can be obtained from Bernoulli's Equation,

$$
C_{p}=\frac{p-p_{\infty}}{\frac{1}{2} \rho U^{2}}=1-\left(\frac{V_{(t o t a l)}}{U}\right)^{2} .
$$

$C_{p}$ is the coefficient of pressure. Once $\sigma_{j}(j=1,2, \ldots, N)$ and $\gamma$ are evaluated from the above operations, the velocities at all the panels are evaluated. Since the normal components of the velocity for all the panels are already set to zero; only tangential components exist, thus

$$
\frac{V_{(t o t a l)}}{U}=\vec{V} \cdot \hat{t}_{i}=\left(V^{t}\right)_{i}, \quad i=1,2, \ldots, N
$$

where $\left(V^{t}\right)_{i}$ is given by Eq. $\underline{(2.58)}$ and when substituted in Eq. $\underline{2.61}$ yields

$$
\left(C_{p}\right)_{i}=1-\left(V^{t}\right)_{i}^{2}, \quad i=1,2, \ldots, N
$$

The two-dimensional aerodynamic coefficients of lift $\left(C_{l}\right)$, drag $\left(C_{d}\right)$ about the leading edge are computed by the integration of the pressure distribution assuming $C_{p}$ exists at each panel. First, the computation is done by integrating forces in the airfoil-fixed coordinate system followed by a rotation to the respective lift and drag directions along and perpendicular to the free stream $(U)$ as follows Teng [87]:

$$
\begin{gathered}
c_{y}=\sum_{i=1}^{N}\left(C_{p}\right)_{i}\left(x_{i+1}-x_{i}\right) \\
c_{x}=-\sum_{i=1}^{N}\left(C_{p}\right)_{i}\left(y_{i+1}-y_{i}\right) \\
C_{d}=c_{x} \cos \alpha+c_{y} \sin \alpha
\end{gathered}
$$

\footnotetext{
${ }^{5} T$ denotes the transpose
} 


$$
C_{l}=c_{y} \cos \alpha+c_{x} \sin \alpha
$$

\subsubsection{Steady $\rightarrow$ Unsteady}

It was essential to understand the numerical methodology to solve steady-state flow over an airfoil before considering the complex unsteady-state. The airfoil surface is again represented by singularity distributions of source strength $\sigma_{j}(j=1,2, \ldots, N)$ and vorticity $\gamma$ which make $(N+1)$ unknowns already, however, in unsteady flow these unknowns are now time-dependent. Hence, a subscript $k$ is introduced as the time-step index so that the flow solutions can be obtained at successive time-steps $t_{k}(k=1,2, \ldots)$. The unknowns are represented as $\left(\sigma_{j}\right)_{k}(j=1,2, \ldots)$ and $\gamma_{k}$.

\subsubsection{Vortex shedding model for inviscid flow}

In general unsteady flow past airfoil is accompanied by corresponding changes in circulating and thus lift for an airfoil. Since the total circulation in the flow field must be preserved according to Helmholtz theorem, any change in circulation must be balanced by an equal and opposite change in vorticity in the wake which causes vortex shedding at the trailing edge of the airfoil. This vortex shedding at the trailing edge takes place using an additional wake element, shed vorticity panel, attached to the trailing edge as shown in Fig. (2.4c). The shed vorticity panel is characterised by its length $\Delta_{k}$, its inclination angle to the $x$-axis by $\theta_{k}$, and a uniform vorticity distribution $\left(\gamma_{w}\right)_{k}$. At time-step $t_{k}$, the overall circulation on the airfoil $\Gamma_{k}\left(\equiv \gamma_{k} l\right)$ with $l$ referring the perimeter of the airfoil, Helmholtz theorem says

$$
\Gamma_{k}+\Delta_{k}\left(\gamma_{w}\right)_{k}=\Gamma_{k-1}
$$

or

$$
\Delta_{k}\left(\gamma_{w}\right)_{k}=\Gamma_{k-1}-\Gamma_{k}=l\left(\gamma_{k-1}-\gamma_{k}\right),
$$

where $\Gamma_{k-1}$ and $\gamma_{k-1}$ represent the overall circulation on the airfoil surface and the vorticity strength, respectively, at time-step $t_{k-1}$.

When marching from $t_{k}$ to $t_{k+1}$, the shed vorticity panel is detached from the trailing edge and convects downstream as a free vortex with circulation $\Delta_{k}\left(\gamma_{w}\right)_{k}$. Simultaneously, a new shed vorticity panel is formed, and the vortex shedding process is repeated. The presence of the shed vorticity panel and the wake core vortices influences upstream flow. As a result, the wake core vortices are convected under the influence of freestream, and singularity distributions surface, including the shed vorticity panel. This coupling process increases the complexity of unsteady flow model. The solution procedure recommended is an iterative one.

In addition to surface singularities $\left(\sigma_{j}\right)_{k}(j=1,2, \ldots)$ and $\gamma_{k}$, there are three additional unknowns $\left(\gamma_{w}\right)_{k}$ and $\theta_{k}$ due to vortex shedding process. The Helmholtz theorem, Eq. (2.69) provides one additional relation in conjunction with flow tangency condition and the Kutta 
condition. Basu and Hancock [76] recommended two additional relations based on their assumptions on the orientation and the length of the shed vorticity panel as

$$
\begin{gathered}
\tan \theta_{k}=\frac{\left(v_{w}\right)_{k}}{\left(u_{w}\right)_{k}}, \\
\Delta_{k}=\left(t_{k}-t_{k-1}\right)\left[\left(u_{w}\right)_{k}^{2}+\left(v_{w}\right)_{k}^{2}\right]^{\frac{1}{2}},
\end{gathered}
$$

where $\left(u_{w}\right)_{k}$ and $\left(v_{w}\right)_{k}$ are the flow velocity components at the panel midpoint in horizontal and vertical directions, respectively. The flow tangency condition remains the same for unsteady problem. However, the Kutta condition now includes a term which represents the rate of change of velocity potential according to unsteady Bernoulli's equation as

$$
\left[\left(V^{t}\right)_{1}\right]_{k}^{2}-\left[\left(V^{t}\right)_{N}\right]_{k}^{2}=2\left[\frac{\partial\left(\phi_{N}-\phi_{1}\right)}{\partial t}\right]_{k}=2\left(\frac{\partial \Gamma}{\partial t}\right)_{k} .
$$

\subsubsection{Solution}

The steps to calculate new $A$ and $B$ influence coefficients are similar to the previous ones, however, as mentioned in the preceding section that the presence of wake core vortices influences the upstream flow thus introducing a new influence coefficient to the equations.

\section{Flow-tangency}

$$
\begin{gathered}
\underbrace{\sum_{j=1, j \neq i}^{N} A_{i j}^{n}\left(\sigma_{j}\right)_{k}}_{\text {panel } \rightarrow \text { panel (source) }}+\underbrace{\gamma_{k} \sum_{j=1, j \neq i}^{N} B_{i j}^{n}}_{\text {panel } \rightarrow \text { panel (vorticity) }}+\underbrace{\left(\vec{V}_{\text {stream }} \cdot \vec{n}_{i}\right)_{k}}_{\text {freestream } \rightarrow \text { panel }} \\
\underbrace{\left.\left(\gamma_{w}\right)_{k}\right)\left(B_{i, n+1}^{n}\right)_{k}}_{\text {vorticity shed panel } \rightarrow \text { panel }}+\underbrace{\sum_{m=1}^{k-1}\left(C_{i, m}^{n}\right)_{k}\left(\Gamma_{m-1}-\Gamma\right)}_{\text {wake vortex } \rightarrow \text { panel }}=0
\end{gathered}
$$

Using Eqs. (2.69) and (2.73), a system of algebraic equations is obtained in the form

$$
A \vec{x}=\gamma_{k} \cdot \vec{b}+\vec{c}
$$

where $A$ is an $(N+1) \times(N+1)$ matrix, $\vec{x}=\left[\left(\sigma_{1}\right)_{k},\left(\sigma_{2}\right)_{k}, \ldots,\left(\sigma_{N}\right)_{k}\right]^{T}, \vec{b}=\left(b_{1}, b_{2}, \ldots, b_{N}\right)^{T}$, and $\vec{c}=\left(c_{1}, c_{2}, \ldots, c_{N}\right)^{T}$. An iterative procedure [87] is followed, which projects the wake vortices downstream associated with some guess values of $\Delta_{k}$ and $\theta_{k}$. This is followed by calculating the influence coefficients needed in Eq. (2.73), and then solving Eq. (2.74) using the same method used to solve Eq. $\underline{(2.59)}$ to obtain $\left(\sigma_{j}\right)_{k}$ in terms of $\gamma_{k}$. Next step is to solve for $\gamma_{k}$ using the Kutta-condition of Eq. (2.72) which is a nonlinear equation with $\gamma_{k}$ as the only unknown. Once $\gamma_{k}$ is known, other unknowns are then calculated and this procedure repeats until the values of $\Delta_{k}$ and $\theta_{k}$ converge. The convergence brings the first iteration to an end and the solver proceeds to the next time-step. 
Pressure and loads The unsteady flow Bernoulli's equation for the pressure coefficients on the airfoil surface can be written as [90]

$$
C_{p}=\frac{p-p_{\infty}}{\frac{1}{2} \rho U^{2}}=\left(\frac{V_{(\text {stream })}}{U}\right)^{2}-\left(\frac{V_{(\text {total })}}{U}\right)^{2}-\frac{2}{U^{2}} \frac{\partial \phi}{\partial t}
$$

Once, $\left(\sigma_{1}\right)_{k},\left(\sigma_{2}\right)_{k}, \ldots,\left(\sigma_{N}\right)_{k}$ and $\gamma_{k}$ are known at $t=t_{k}, V_{k}^{t}$ is computed in a similar fashion as explained in sec. (2.6.1),

$$
\begin{aligned}
{\left[\left(V^{t}\right)_{i}\right]_{k}=} & \sum_{j=1, j \neq i}^{N} A_{i j}^{n}\left(\sigma_{j}\right)_{k}+\gamma_{k} \sum_{j=1, j \neq i}^{N} B_{i j}^{n}+\left(\vec{V}_{\text {stream }} \cdot \vec{t}_{i}\right)_{k} \\
& \left.+\left(\gamma_{w}\right)_{k}\right)\left(B_{i, n+1}^{n}\right)_{k}+\sum_{m=1}^{k-1}\left(C_{i, m}^{n}\right)_{k}\left(\Gamma_{m-1}-\Gamma\right),
\end{aligned}
$$

thus enabling $C_{p}$ to be calculated as

$$
\left[\left(C_{p}\right)_{i}\right]_{k}^{2}=\left[\left(V_{(\text {stream })}\right)_{i}\right]_{k}^{2}-\left[\left(V^{t}\right)_{i}\right]_{k}^{2}-2 \frac{\left(\phi_{i}\right)_{k}-\left(\phi_{i}\right)_{k-1}}{t_{k}-t_{k-1}}
$$

The force coefficients, $\left(C_{l}, C_{d}\right)$, are then calculated in the same way as it is done in for the steady flow problem (sec. $(\underline{2.6 .1})$ ).

\subsection{Validation}

Once the strength of the sources $\sigma_{j}$ and vorticity $\gamma$ over the airfoil surface is known, the velocity at each collocation point is computed using Eq. (2.76) enabling the calculation of pressure coefficient using Eq. (2.77). In the proceeding subsections, the numerical results for steady and unsteady flow simulations over a two-dimensional NACA0012 airfoil is presented.

\subsubsection{Airfoil in a steady flow}

The steady pressure distribution over a two-dimensional NACA 0012 airfoil is first examined. NACA 0012 is chosen since there exists a vast literature, both numerical and experimental, on its steady and unsteady aerodynamic characteristics. Moreover, NACA 0012 is widely used in the validation studies of aeroacoustic solvers, which makes it an ideal choice for the present study.

The generation of lift on a low-speed airfoil is inherently linked with the formation of starting vortex which forms at the trailing edge [91]. In body-fixed reference frame, the problem of an impulsively starting airfoil is equivalent to that with a background flow abruptly accelerating from zero to a constant. At $t>0$ the flow can be considered as a steady flow, yet the problem is itself an unsteady one because of the formation of a starting trailing-edge vortex. But once this trailing-edge vortex has convected far downstream, the lift force saturates and becomes steady and constant. Wagner [5] studied this problem and provided a function using Betz's method, famously known as Wagner function that concerns the growth of circulation or lift about an airfoil at a small fixed angle of attack starting impulsively from rest to a uniform velocity. Fig. $\underline{(2.5)}$ shows the responses of a flat plate and a NACA 0001 profile solved numerically using 


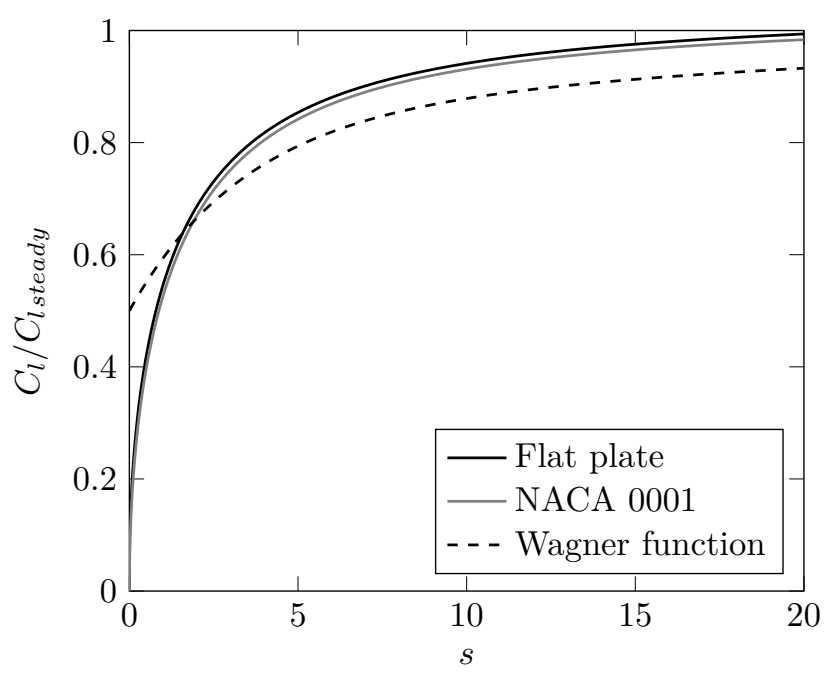

Figure 2.5: Comparison of Wagner's function with the numerical predictions for a flat plate and a NACA 0001 profiles.

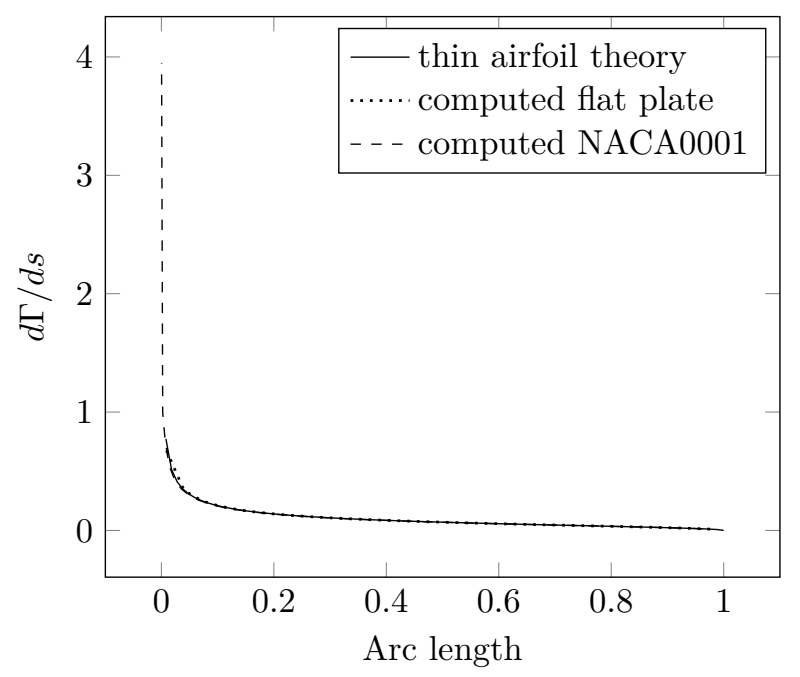

Figure 2.6: Development of circulation over chord length

the present approach. The responses are evidently very close to Wagner's function for the flat plate.

Fig. (2.6) shows the development of circulation over chord length of the profiles. The results are compared to the analytical solutions of thin-airfoil theory [92].

The two-dimensional steady flow problem for NACA0012 is solved at various angles of attack to obtain the pressure characteristics of the airfoil using present method. Fig. (2.7) shows a schematic of the physical problem where a NACA0012 airfoil is placed in a steady flow with

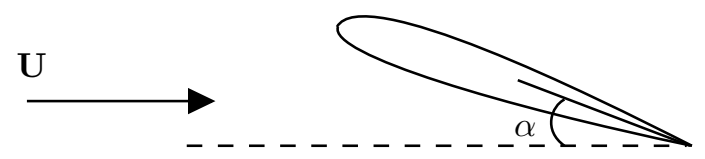

Figure 2.7: Schematic of a NACA0012 airfoil at an angle of attack, $\alpha$, in a steady flow, $U$ 
the free stream velocity of $U$ at an angle of attack, $\alpha$. The upper and the lower surfaces of the airfoil are discretised into 50 chordwise panels. The total simulation time is $10 \mathrm{~s}$ with a time-step of $0.01 \mathrm{~s}$ which allows the starting vortex to convects past to 10 chord lengths downstream of the airfoil since the total time elapsed is $10 \mathrm{~s}$, the free-stream velocity is $U=1$ $\mathrm{m} / \mathrm{s}$, and the total chord length is $1 \mathrm{~m}$. This facilitates convecting the starting vortex to the far-field, which is an essential condition to validate the results.

In Fig. (2.8), the pressure distributions over the NACA0012 airfoil at different angles of attack are shown.

It is readily evident in Fig. (2.8a) that at $\alpha=0^{\circ}$, the lift generated is zero because the negative pressure coefficient is plotted along the positive $y$-axis, and the pressure coefficient is observed to stagnate at the leading edge $(C p=1)$.

A comparison between the numerical results of the present approach and numerical results of xfoil are shown in Fig. (2.8a)-(2.10d). Consequently, the pressure is observed to drop as the flow accelerates symmetrically over the top and bottom surfaces until a minimum pressure of $C p=-0.5$ is reached around $x / c_{a}=0.2$ near the maximum thickness. A prominent leading-edge suction peak is also observed for the cases with $\alpha>0$ that grows with increasing $\alpha$. A remarkable agreement between the numerical results and xfoil results over a range of angles of attack from $\alpha=0-10^{\circ}$ is achieved.

Fig. (2.9) a depicts the variation of lift coefficient, $C_{l}$, of the NACA0012 airfoil for angle of attack $\alpha \in\left(0^{o}, 2^{o}, \ldots, 10^{\circ}\right) . C_{l}$ is plotted on the vertical axis and $s=x / c_{a}{ }_{-}$on horizontal. The $C_{l}$ values seem to saturate with time which corresponds to the transition of flow around the airfoil from unsteady to steady. This data is compared with the numerical data of xfoil for NACA0012, as shown in Fig. (2.9b) and found to be in good agreement over a range of $\alpha$ from $0^{\circ}$ to $10^{\circ}$ degrees. The stall characteristics are not captured because the present model does not account for flow separation.

\subsubsection{Airfoil with an unsteady motion}

The accuracy and applicability of the developed unsteady solver are examined in this section. The analysed case compare the results of the developed unsteady panel code with a known analytical solution. This helps to establish the accuracy and applicability of the developed code prior to its application to the aeroacoustic problems. The code is validated by simulating a series of cases in which NACA0012 is undergoing unsteady motion.

A two-dimensional NACA0012 airfoil performing a pitching motion relative to the freestream flow is simulated using the unsteady code for reduced frequency of $\mathrm{k}=0.25$ and 0.75 , and amplitudes of $\bar{\alpha}=2$ and 4 degrees. The parameters used are $c_{a}=1 \mathrm{~m}, U=1 \mathrm{~m} / \mathrm{s}$. The pivot for the pitching motion is fixed at quarter chord from the leading edge of the airfoil. For all the simulations, the chord length is fixed at $c_{a}=1 \mathrm{~m}$. Fig. (2.10) compares the lift coefficient from the numerical simulation to the Theodorsen solution. The results demonstrate that for airfoil performing only pitching motion at small reduced frequencies, airfoil thickness induces a small phase difference between the numerical and Theodorsen lift solutions. These results also support the fact that Theodorsen solution is mainly applied to thin-airfoil, and the discrepancies in the phases are expected in thicker airfoils such as NACA 0012.

\footnotetext{
${ }^{6} s=x / c_{a}$ is the total distance travelled by the airfoil normalised to the chord length
} 


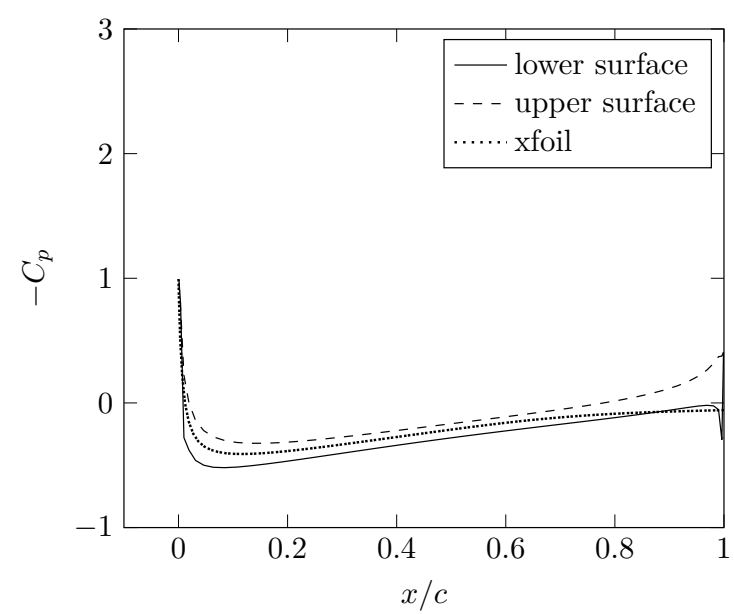

(a) $\alpha=0^{\circ}$

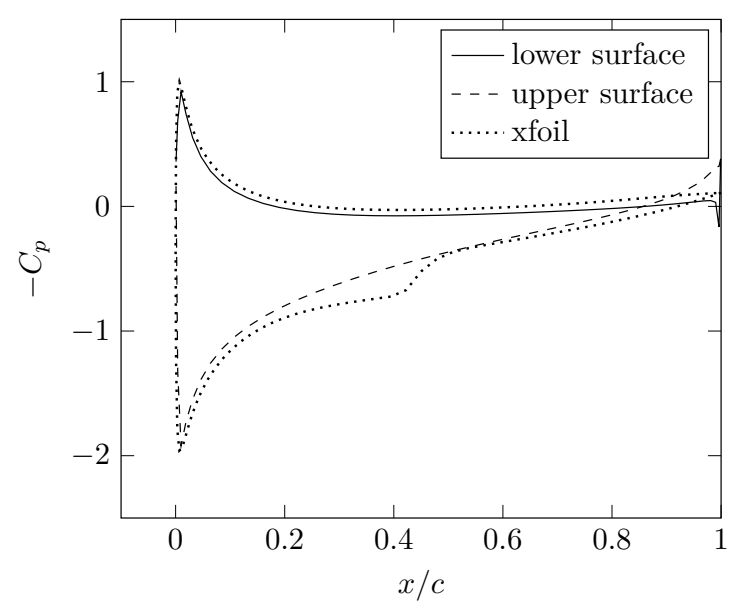

(c) $\alpha=5^{\circ}$

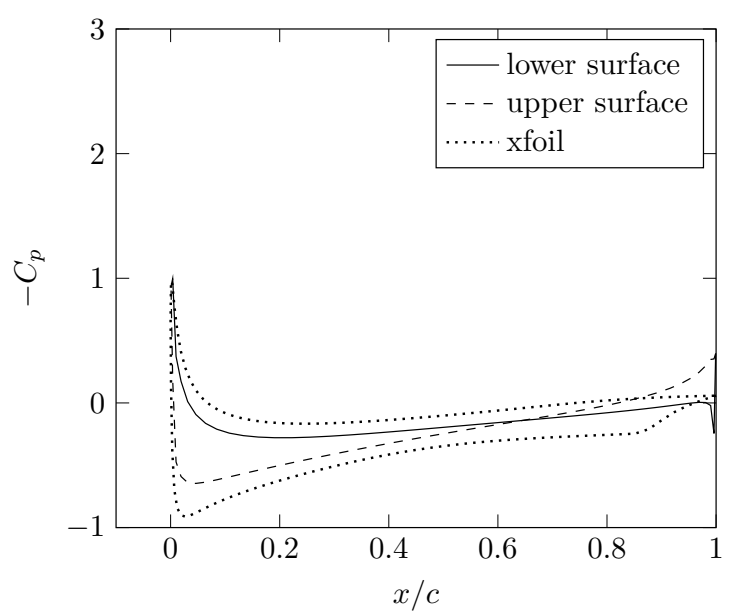

(b) $\alpha=2^{o}$

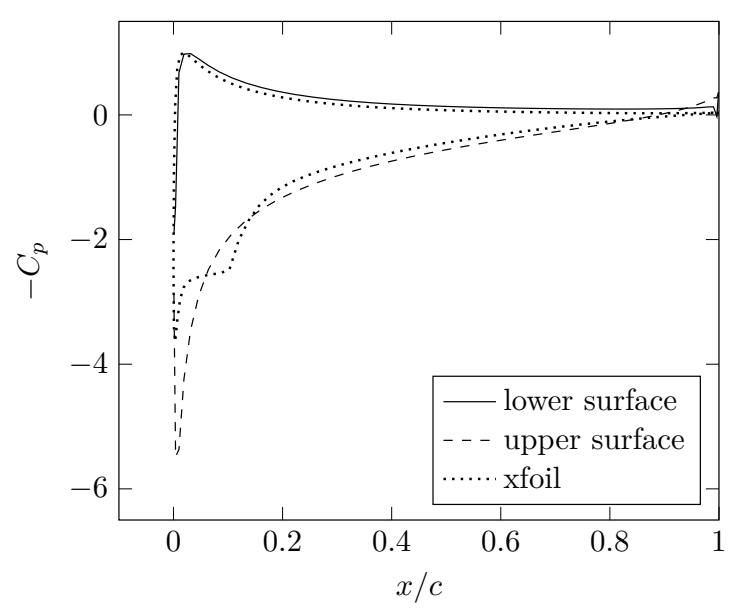

(d) $\alpha=10^{\circ}$

Figure 2.8: $C_{p}$ distributions for the NACA0012 airfoil for the indicated angles of attack

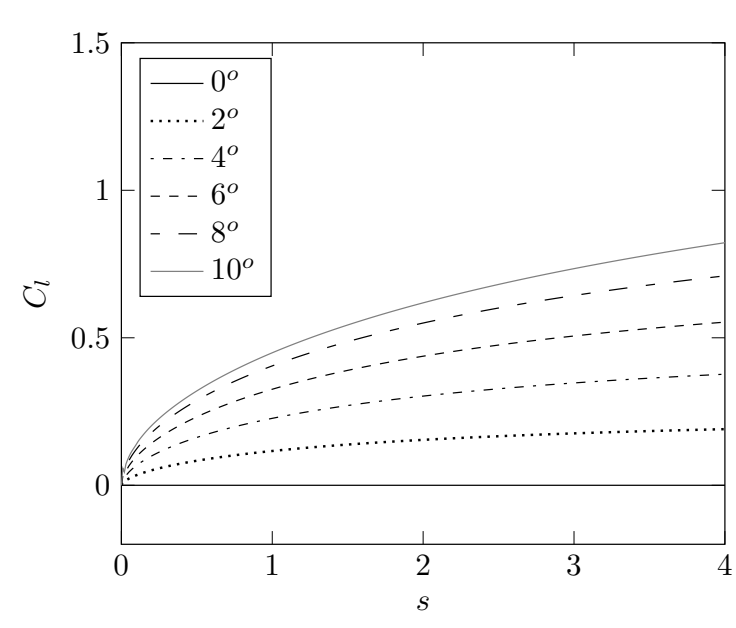

(a)

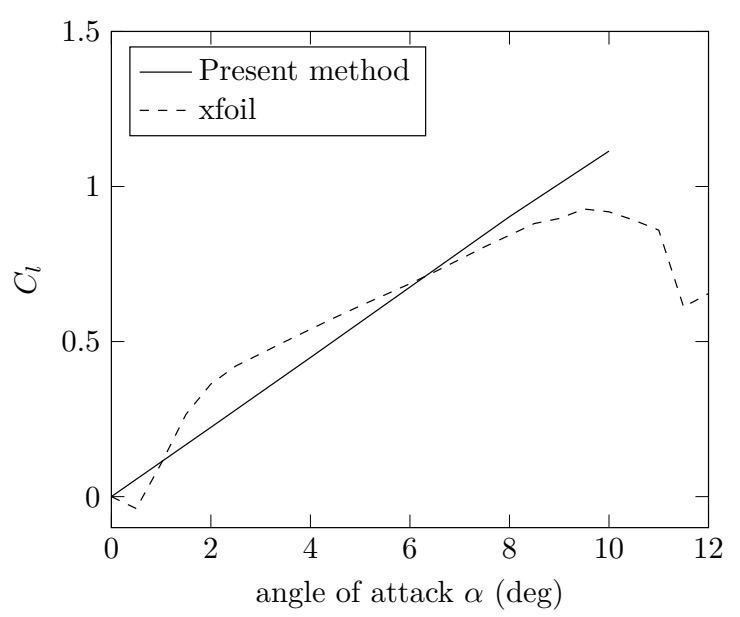

(b)

Figure 2.9: Lift coefficient $\left(C_{l}\right)$ vs angle of attack $(\alpha)$ for a NACA0012 airfoil

Next, a NACA 0012 airfoil with a combined pitching and heaving motion, adapted from the experiment conducted by Schouveiler, Hover, and Triantafyllou [93] and numerical simulations by Xia and Mohseni [94], is considered. The parameters used are $c_{a}=1 \mathrm{~m}, U=1 \mathrm{~m} / \mathrm{s}$, 


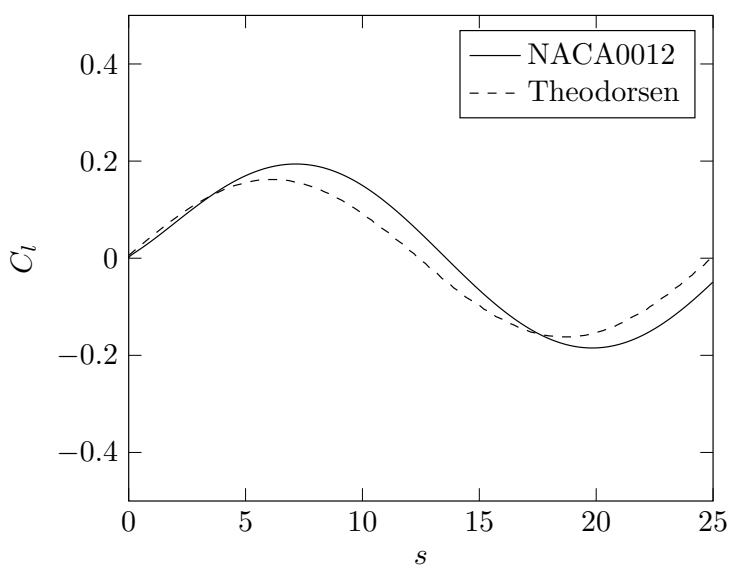

(a) $\mathrm{k}=0.25$ and $\bar{\alpha}=2^{\circ}$

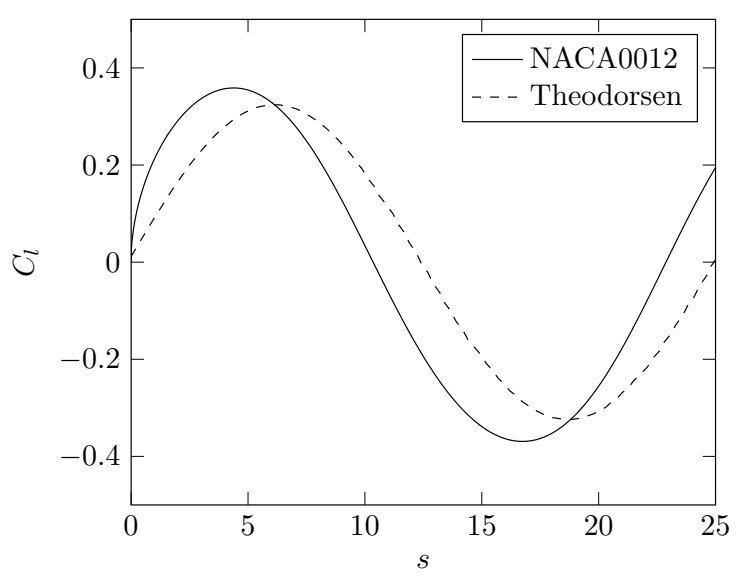

(c) $\mathrm{k}=0.25$ and $\bar{\alpha}=4^{\circ}$

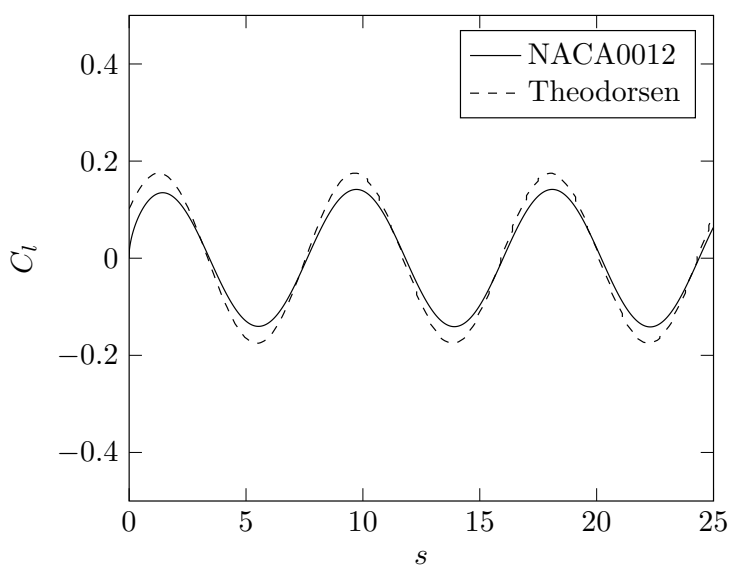

(b) $\mathrm{k}=0.75$ and $\bar{\alpha}=2^{\circ}$

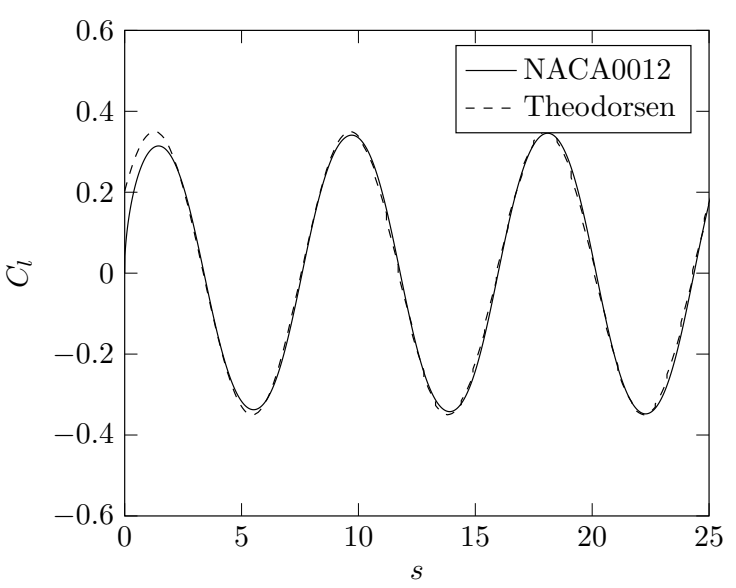

(d) $\mathrm{k}=0.75$ and $\bar{\alpha}=4^{\circ}$

Figure 2.10: Reduced frequency $(k)$ vs. lift coefficient $\left(C_{l}\right)$ for a NACA0012 airfoil performing pitching motion with an amplitude of $\bar{\alpha}=2^{\circ}$

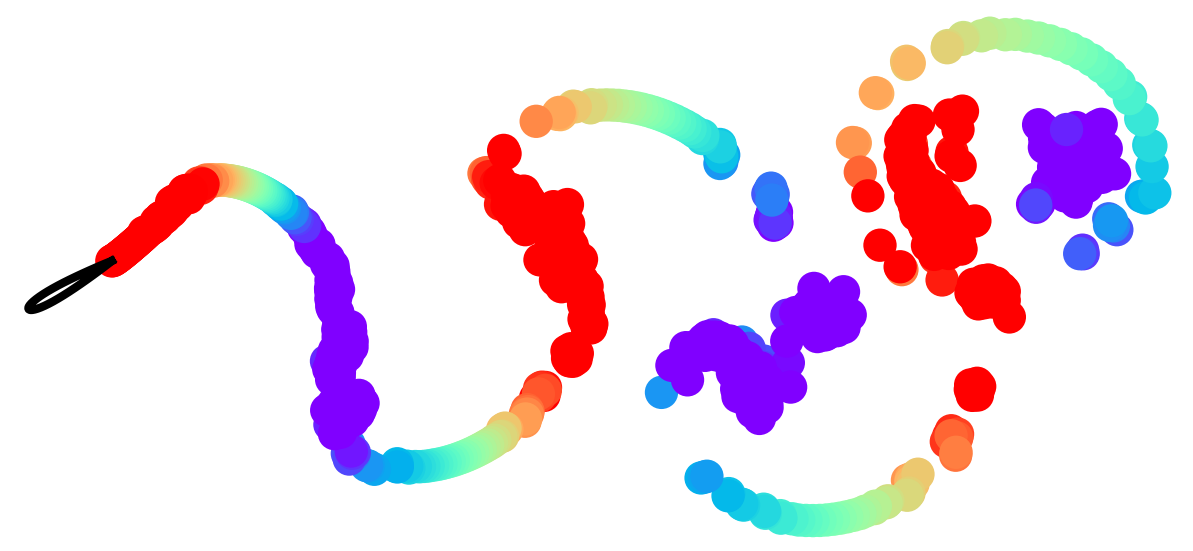

Figure 2.11: NACA 0012 airfoil undergoing pitching and heaving, simultaneously 


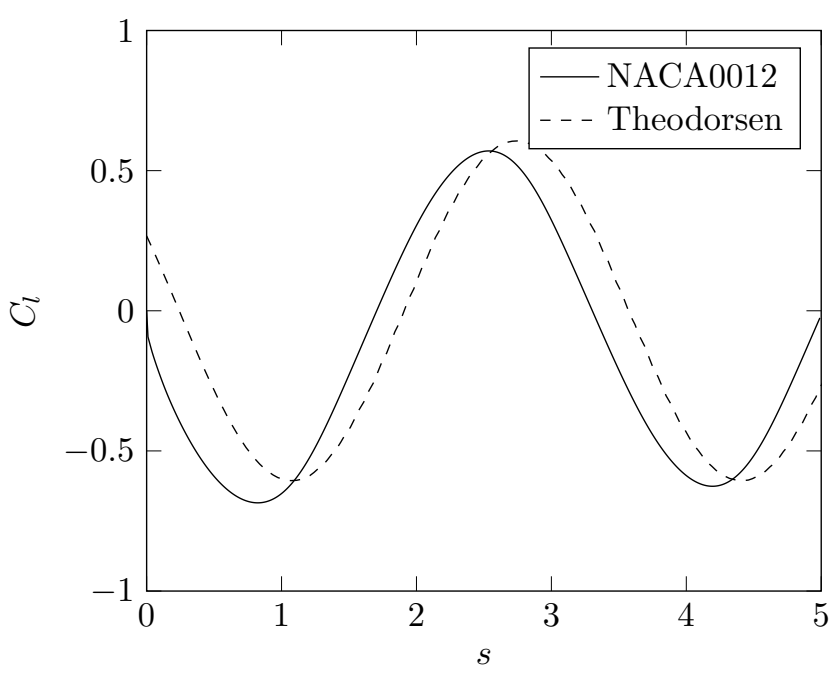

Figure 2.12: Unsteady lift coefficient of NACA0012 airfoil undergoing pitching and heaving motion, simultaneously

$h_{o} / c_{a}=0.2$ with $S t=0.3, \mathrm{k}=3 \pi / 10$. The airfoil is discretised into 50 chordwise boundary elements for both the top and bottom surfaces, and the computation is carried out ove 100 times steps per oscillation cycle for 2 cycles. Fig. (2.11) shows the simulated flow field displaying the vortical structures in the wake. Fig. (2.12) compares the time-varying lift coefficients of the analytical and numerical solutions. A slightly lower peak lift coefficient is observed in the numerical solution compared to the Theodorsen model, which is also observed in other studies [95]. 


\section{3}

Vortex Generated Turbulence

Sparsh Sharma and Ennes Sarradj. "Two-dimensional isotropic turbulent inflow conditions

for vortex particle method". In:Physical Review Fluids4.2 : (Feb. 2019)

Vortex methods are based on the discretisation of the vorticity field and the Lagrangian description of the governing equations. The method has been in use since the late 1930s when Rosenhead calculated the Kelvin-Helmholtz instabilities by hand. The vortex particle method in three-dimensions was primarily derived from the two-dimensional method and was initially using vortex filament approximations for accounting the domain vorticity. Vortex methods provide excellent results for the simulations of bluff body aerodynamics at a fraction of the computational cost of classical Eulerian methods [96]. An elaborate review of the history of vortex methods is given by Yokota and Obi [97] where vital contributions of Ould-Salihi, Cottet, and El Hamraoui [98], Hamada et al. [99], Rosenhead and John [100], Chorin and Bernard [101], Hald, Mauceri, and Prête [102], Beale and Majda [78], and Hess and Smith [84] are discussed thoroughly. The major shortcoming of vortex filaments and sheet method is its requirement of storing the convection memory of the particles. In addition to the memory requirements, the vortex particle methods require the evaluation of an N-body interaction problem at each iteration. The particles convect ${ }_{-}^{1}$ in a Lagrangian fashion in which their positions change at each time-step which essentially require the re-computation of particle-particle influences. Development of fast algorithms such as the fast multipole method (FMM) by Greengard and Rokhlin [103] and the treecode by Barnes and Hut [104] were applied to vortex method calculations to accelerate them. This reduced the calculation cost from $O\left(N^{2}\right)$ to $O(N)$ and $O(N \log N)$ for FMM and treecode, respectively.

\subsection{Vortex methods in aeroacoustics}

Acoustic radiation from a body immersed in a turbulent flow field, popularly known as leading-edge noise, is a widely studied topic in Computational Aeroacoustics (CAA). The

\footnotetext{
${ }^{1}$ convection and advection are synonymous in the absence of molecular diffusion
} 
early studies $[60,61,62]$ show the use of frequency domain analysis in which the incoming disturbance is modelled as a series of harmonic gusts by analysing the fields Fourier-transform. Studies based on this method have accounted for the effects of thickness, camber, and angle of attack on noise generation, but were mainly used for relatively low frequencies. As the frequency increases, the spatial and temporal resolution requirements make these numerical methods more difficult to apply [63]. Another prevalent method in use is the time-domain analysis $[64,65,66]$ which is based on vortex methods and uses a set of discrete vortices to realise the turbulent inflow. Gennaretti, Luceri, and Morino [35] formulated a boundary integral formulation for the unified aerodynamic and aeroacoustic analysis of rigid lifting bodies in arbitrary motion. Numerical results concerning hovering and advancing rotor configurations have been presented in order to demonstrate the capability of the methodology to capture aerodynamic and aeroacoustic solutions. However, numerical application of potential theory, with its singular representation of vortical regions, would not yield a proper solution in the case of close wake-body interactions. In 2007, Gennaretti and Bernardini [44] revised their earlier approach by assuming the vortex to have a finite core in which the induced velocity remains bounded which yields a regular influence, even at body or wake points that approach it. These studies were mainly dealing with the Blade-Vortex Interaction (BVI) where the statistical modelling of the inflow due to which the stochastic nature of the disturbance originates was missing. In more recent papers, Glegg [67] used a time-domain method where the statistical nature of the disturbance was modelled using discrete vortices. Both methods, frequency domain and time-domain, have their advantages and limitations which are presented by Grace [68]. She concluded that the time-domain analysis has an advantage over the frequency domain, primarily due to the ability to realise the turbulent inflow with the desired statistical properties.

In classical panel method, widely used in aerodynamics, vorticity is limited to vorticity sheet and filament approaches. Vortex particle and panel method have been used predominantly in two-dimensional unsteady aerodynamics problems and extended to the third dimension for lifting surface problems like wind turbines. In recent times, the vortex particle methods have mainly been used to create turbulent inflow ${ }_{-}^{2}$ conditions for Large-Eddy Simulations $\underline{[105}$, $\underline{106}, \underline{107}, 54,56,108]$. These studies were intended to study the aeroacoustic behaviour of an airfoil in a turbulent flow field. In vortex methods, a very few studies have accounted for turbulence modelling in vortex methods, e.g. Pereira, Hirata, and Filho [109] in turbomachinery, Rasmussen et al. [110] and Hejlesen et al. [111] in bridge cross-sections. These studies were restricted to two-dimensions only.

On the other hand, it is far more challenging to model the inflow conditions using a set of discrete vortices [112], since the parameters which control the size and the strength of these vortices are unknown. These inflow conditions can be imposed by methods which are considerably expensive like those based on proper orthogonal decomposition which requires appropriate direct numerical simulations [113] or experimental data [114] to obtain the energetic modes. Different methods are available to synthesise a turbulent signal without involving the solution to Navier-Stokes equations, and one such way is by superimposing a white noise signal over the mean flow. Chapman [115], Benzi [116], and Elhmaidi [117] used a similar

\footnotetext{
${ }^{2}$ synthetic turbulence approach (also referred to as synthetic eddy method)
} 
method to realise a two-dimensional synthetic turbulent inflow using point vortices. Esler [118] studied the behaviour of a finite number of point vortices and examined the possibility of replacing a turbulent flow with a collection of point vortices. However, there is a problem with approximating the vorticity with point vortices (delta functions), i.e. infinite spikes, which introduces an associated singularity in the streamfunction and velocity fields. This singularity appears when the distance to the point vortex becomes very small.

\subsection{Vorticity and turbulence}

"What is turbulence?" von Kármán [119] in his lecture defined turbulence by stating it as an irregular motion which in general makes it appearance in fluids when they flow past a solid surface or even when nearby streams of same fluid flow past or over one another. This definition is not entirely accurate since there are fluids which are irregular but can not be considered as turbulent. Later, Hinze [120] proposed a new definition of turbulence that the fluid is considered turbulent only if various quantities show a random variation in the Spatio-temporal system so that statistically distinct averages can be discerned.

Turbulence is chaotic, and an essential feature of turbulent flows is that they are rotational, i.e. they have non-zero vorticity. The vorticity is given by $\omega$, which is the curl of the velocity as in Eq. (2.3) and is equal to twice the rate of rotation of the fluid at a point in space-time coordinate system.

\subsubsection{Sources of complexity in fluid}

The simplest of all fluids is irrotational incompressible flow in which the velocity derives from a potential. These flows are governed by Laplace's equation where the solutions are smooth, and where the time dependence is not explicit. The solution strongly depends upon the boundary conditions, and if the boundary conditions are smooth, so is the flow. There is no chaos in these flows. Nevertheless, this uncomplicated behaviour can be broken by lifting the incompressibility assumption, which leads to acoustics [121], or by not considering the fluid irrotational, which leads to turbulence. The mathematical background for the flows involving vorticity is presented in the previous chapter. The general mechanism of such a flow is then that the vorticity is transported by the velocity, while the resulting vorticity distribution determines the velocity through Poisson's equation Eq. (2.20).

Comment: This thesis studies vorticity in two-dimensional flow. This is significant by itself, even independently of turbulence, because vorticity is an expression of angular momentum of the fluid, and angular momentum, being a conserved quantity, determines in large part how the fluid moves. The limitation of two-dimensional flows is artificial, and its effects can be significant. Most flows of practical concern are three-dimensional, and vorticity acts quite differently in three dimensions than in two. It is simpler to examine them in two-dimensions, which will help later to understand the three-dimensional effects.

It is beneficial to discuss turbulence and its characteristics before using vortices to generate a turbulent inflow for the numerical method. 


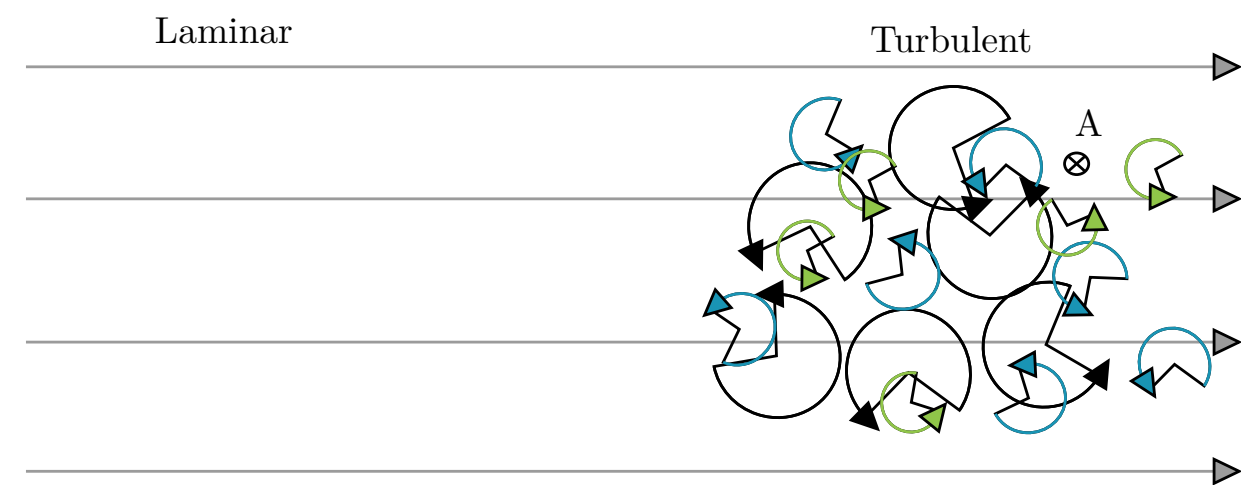

Figure 3.1: Transitioning from laminar flow to turbulent one; straight, parallel grey lines are streamlines, which are everywhere parallel to the mean flow.

\subsection{Turbulence}

Turbulent flows are identified by random and unpredictable behaviour, irregular patterns, and the existence of numerous space and time scales. The properties like velocity vector, pressure, vorticity, density, etc., undergo random fluctuations created by the presence of numerous eddies of various sizes and, as a result, vary extremely intermittently in space in time, with broad frequency ranges. This unpredictability and the existence of a broad range of scales have their origin in the nonlinear behaviour of the Navier-Stokes equations. They develop when the source of kinetic energy responsible for driving the fluid in motion is strong and able to overcome the viscosity effects that slow down the fluid. When this is not the case, and viscosity predominates, the flow is said to be laminar, and hence regular and predictable. The energy source in a flow can be of various natures: pressure gradients in a pipe flow, initial impulsion for jets, or temperature differences in heated flows where buoyancy takes place [122]. Reynolds number, $R e$, the parameter is commonly used to characterise a flow laminar or turbulent. It is a measure of the ratio of inertial forces to viscous forces. The inertial forces are proportional to $\rho U^{2}$, and the viscous forces are proportional to an expression like $\mu \partial u / \partial y$, or approximately to $\mu U / l$, for a given geometry. Hence, the Reynolds number can be expressed as

$$
R e \equiv \frac{\rho U^{2}}{\mu U / l}=\frac{\rho U l}{\mu} .
$$

Turbulence appears when the Reynolds number measuring the relative strength of inertial and viscous forces must surpass a specific threshold. However, determining this value is not a straightforward task, as it takes some time and needs some space for turbulence to develop. Moreover, there is no general transition scenario, even for a given class of flows [123].

\subsubsection{Consequences of turbulence}

The presence of turbulence in any fluid flow facilitates a very efficient transport of mass, momentum, and heat. Characteristics like increase in diffusion in a given geometry, expansion of the free boundaries of the flow, reduction of flow separation regions, an increase in thermal exchanges are few desirable consequences of turbulence.

The present work is interested in one of the most undesirable and inevitable consequences of turbulence, acoustic radiation. This noise generated is the result of turbulence and its 


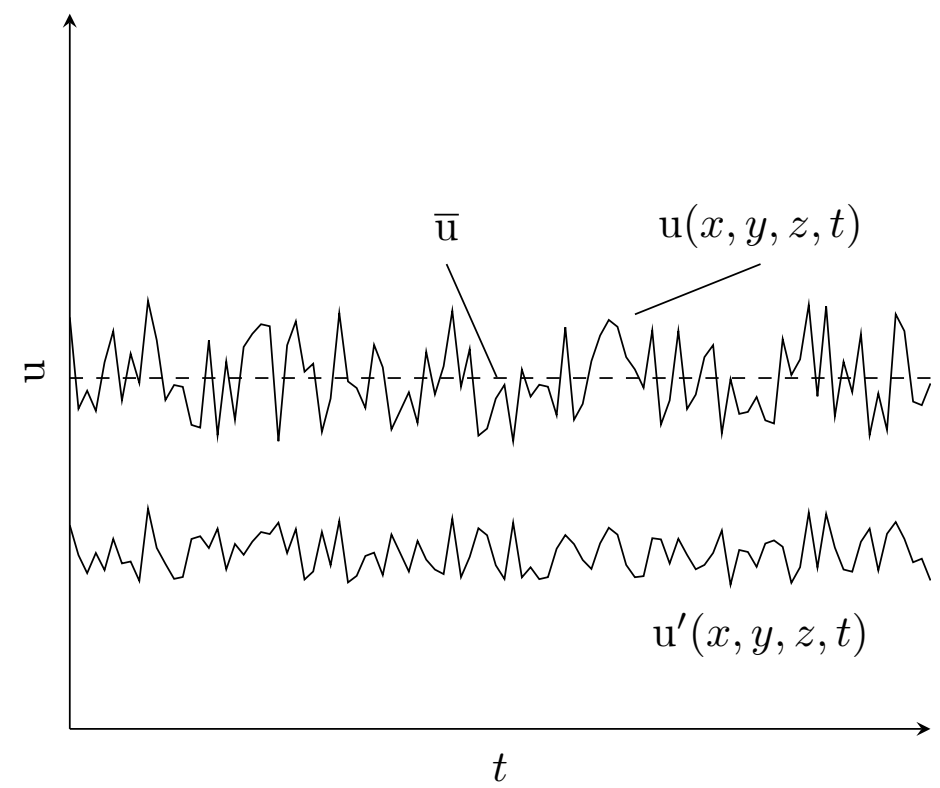

Figure 3.2: Reynolds decomposition of the velocity recorded at point A

interaction with bodies, and therefore arises in nearly every engineering application. Because of its stochastic nature, it has a broad frequency spectrum. Turbulent energy is converted into acoustic energy most efficiently near sharp edges. Turbulence noise exists almost every time. Consequently, aerodynamic noise is usually a broadband noise sometimes augmented by narrow tonal components coming from impulsive noise sources.

\subsubsection{Measures of turbulence - Intensity}

Turbulent eddies generate fluctuations in velocity. As an example, the velocity measured at point A in Fig. (3.1), which shows random eddies in a turbulent flow. The velocity in turbulent flow includes a mean and a turbulent or fluctuating component as

$$
\mathrm{u}(x, y, z, t)=\overline{\mathrm{u}}+\mathrm{u}^{\prime}(x, y, z, t),
$$

where $\overline{\mathrm{u}}$ is the mean velocity and $\mathrm{u}^{\prime}$ is the fluctuating component in the total velocity. Fig. (3.2) shows the so-called Reynolds decomposition of the velocity. By the definition of a mean, the average value of $\mathrm{u}^{\prime}$ will be zero; however, the mean of the squares of the fluctuating components is not equal to zero, because all are positive. For the horizontal component of the velocity, $u$, the mean square can be written as

$$
\overline{\left(u^{\prime}\right)^{2}}=\lim _{n \rightarrow \infty}(1 / n) \sum_{i=1}^{n} u_{i}^{\prime 2} .
$$

The root-mean-square of the above quantity, i.e. the measure of magnitude of the velocity fluctuations about the mean value is called the intensity of turbulence.

$$
T u=\frac{1}{\overline{\mathrm{u}}} \sqrt{\frac{\overline{u^{\prime 2}}+\overline{v^{\prime 2}}+\overline{w^{\prime 2}}}{3}} .
$$


For the case of isotropic turbulence, the three fluctuating velocity components are identical and Eq. (3.4) reduces to

$$
T u=\frac{\sqrt{\overline{u^{\prime 2}}}}{\overline{\mathrm{u}}} .
$$

Turbulence intensity $T u$ is related to the mean kinetic energy of turbulence per unit mass, $k$, as

$$
k=\left(\frac{3}{2} \overline{\mathrm{u}}^{2}\right) T u^{2}
$$

\subsubsection{Measures of turbulence - Scale}

Fluctuations of instantaneous flow characteristics (pressure, velocity, vorticity) depend on both space and time; they occur over a vast range of scales (whether interpreted in terms of time or distance). The smaller scales are settled by the fluid viscosity while the largest is the most often limited by the space available to the flow [124]. For turbulent flow, the size of the largest eddies is given by the characteristic length scale associated with the characteristic geometry length, $\lambda$, and known as integral length scale, and the smallest eddy size is given by the so-called, Kolmogorov length scale, $\eta$. The smallest length scales in the turbulent flow are those where the kinetic energy is dissipated into heat energy. The dissipation rate is independent of viscosity, but the scales at which this energy is dissipated depends upon both the dissipation rate and viscosity. Hence, an estimate for such scales is represented as

$$
\eta=\left(\frac{\nu^{3}}{\epsilon}\right)^{1 / 4}
$$

where $\eta$ is called the Kolmogorov length scales, the dissipation rate per unit mass is denoted by $\epsilon$ and viscosity by $\nu$.

Kolmogorov length scales are related to the largest length scales in the flow as

$$
\frac{\lambda}{\eta} \sim\left(\frac{U \lambda}{\nu}\right)^{3 / 4}=R e^{3 / 4}
$$

where $R e$ is the Reynolds number based on the large scale flow features. From now, the mean velocity $\overline{\mathrm{u}}$ will be denoted by $U$. Another regularly encountered length scale is known as Taylor micro-scale and gives a reasonable estimate for the fluctuating strain rate field.

Next is the time scale, which is the time taken by an eddy to pass all of its energy to smaller scales, known as the eddy turnover time. If $\lambda$ is the length scale and of the typical eddy then the turnover time is given by

$$
t_{\lambda}=\frac{\lambda}{U}
$$

The time scales for the small eddies can also be derived using the viscosity and dissipation,

$$
t_{\eta}=\left(\frac{\nu}{\epsilon}\right)^{(1 / 2)}
$$


Similar to an interesting relation between the length scales given in Eq. (3.8), a relation between the time scales can also be derived as

$$
\frac{t_{\lambda}}{t_{\eta}}=R e^{1 / 2}
$$

\subsubsection{Measures of turbulence - The Energy Spectrum}

As mentioned in preceding sections, turbulent fluctuations are composed of a wide range of length and time scales. Fig. (3.1) can be referred for the visualisation of eddies of different sizes symbolising to the presence of different length scales in a turbulent flow field. It is convenient to use Fourier series to analyse turbulence [125]. The range of scales in a turbulent flow stretches from the largest scales which interact with the mean flow to the smallest scales where dissipation happens. The large eddies have large fluctuating velocities, which implies high kinetic energy, $u_{i}^{\prime} u_{i}^{\prime} / 2$. In wavenumber space, the energy of eddies can be expressed as

$$
E(\kappa) d \kappa
$$

which depicts the energy contribution from the scales with wavenumber between $\kappa$ and $\kappa+d \kappa$ to the turbulent kinetic energy $k$. The total kinetic energy is calculated by integrating the above equation over the whole wavenumber space as

$$
k=\int_{0}^{\infty} E(\kappa) d \kappa
$$

Eq. (3.13) can be seen as a way to calculate the kinetic energy by sorting all the eddies by size (i.e. wavenumber), then computing the kinetic energy of each eddy size (i.e. $E(\kappa) d \kappa)$, and finally summing the kinetic energy of all the eddy sizes. The physical meaning of $E$ is the kinetic energy per unit wavenumber of eddies of size $l_{\kappa}^{3} \propto \kappa^{-1}$.

\subsection{Homogeneous and Isotropic Turbulence}

A turbulent flow field is said to be homogeneous if all statistics are invariant under a spatial translation. Then, the one-point statistical averages, such as $\overline{u_{1}^{\prime 2}}$ or $\overline{u_{1}^{\prime} u_{2}^{\prime}}$ are not dependent on the observation point. In a similar way, if this homogeneous field is statistically invariant under rotations and reflections of the coordinate system, it is called isotropic . $_{\text {. }}$.

No real turbulent flow can be precisely isotropic. Turbulence can be completely isotropic if it occurs in a fluid occupying an infinite space whereas any real flow must have a boundary [128].

\footnotetext{
${ }^{3} l_{\kappa}$ : length scale of a turbulent eddy

${ }^{4}$ The concept of isotropic turbulence is mathematical idealisation, which is convenient only for the approximate description of certain distinctive types of turbulent flow. The isotropy conditions are satisfactorily fulfilled for a particular class of turbulent flows produced in laboratory wind tunnels. Such flows are of lesser importance in practice; however, for a theoretical study, the case of homogeneous and isotropic turbulence is very attractive. It is mathematically the most uncomplicated, thus ideal to begin with and try to use it to exhibit some of the characteristic properties of real turbulent flow. Kolmogorov [126] showed that many of the ideas and methods of the theory of isotropic turbulence by Taylor [127] can be successfully transplanted into a more general theory.
} 


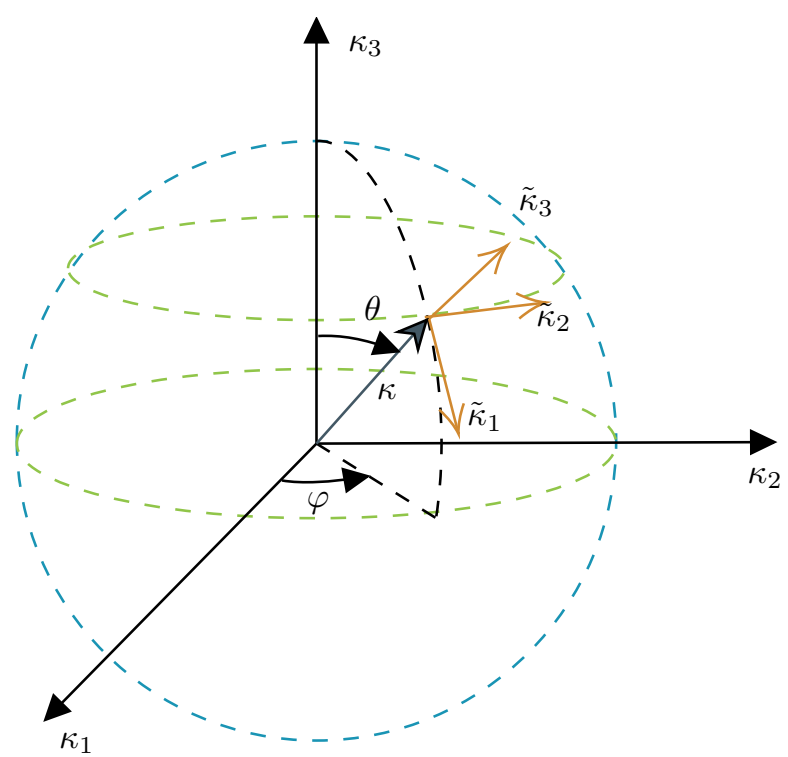

Figure 3.3: Basic frame $\left(\kappa_{1}, \kappa_{2}, \kappa_{3}\right)$ and local frame $\left(\tilde{\kappa}_{1}, \tilde{\kappa}_{2}, \tilde{\kappa}_{3}\right)$ in the Fourier space. The Fourier contribution $\hat{\boldsymbol{u}}(\boldsymbol{\kappa})$ is in the plane $\left(\tilde{\kappa}_{1}, \tilde{\kappa}_{2}\right)$. The orthonormal local frame, also known as the CrayaHerring frame, is defined by analogy with spherical coordinates, $\tilde{\kappa}_{3}$ is along the wavenumber $\kappa$, $\tilde{\kappa}_{1}$ is tangent to the meridian circle with increasing $\theta, \tilde{\kappa}_{1}$ is tangent to the parallel circle with increasing $\varphi[122]$

\subsubsection{Correlations and spectra}

The statistic containing some information on the spatial structure of a single variable $u^{\prime}(t)$ is the two-point, one-time correlation ${ }_{-}^{5}$ function

$$
\mathrm{R}_{i j}(\boldsymbol{x}, \boldsymbol{r}, t)=\left\langle u_{i}^{\prime}(\boldsymbol{x}, t) u_{j}^{\prime}(\boldsymbol{x}+\boldsymbol{r}, t)\right\rangle=\mathrm{R}_{i j}(\boldsymbol{r}, t) .
$$

The correlation function $\mathrm{R}_{i j}(\boldsymbol{r}, t)$ is independent of $\boldsymbol{x}$, and the information it contains can be re-expressed in terms of the wavenumber spectrum. The spectral tensor of the velocity field $\phi_{i j}$ is defined as the Fourier transform of the correlation function $\mathrm{R}_{i j}(\boldsymbol{r}, t)$, i.e.

$$
\left\{\begin{array}{c}
\phi_{i j}(\boldsymbol{\kappa})=\frac{1}{(2 \pi)^{3}} \int_{\mathbb{R}^{3}} \mathrm{R}_{i j}(\boldsymbol{r}) e^{-i \boldsymbol{\kappa} \cdot \boldsymbol{r}} d \boldsymbol{r} \\
\mathrm{R}_{i j}(\boldsymbol{r})=\int_{\mathbb{R}^{3}} \phi_{i j}(\boldsymbol{\kappa}) e^{i \boldsymbol{\kappa} \cdot \boldsymbol{r}} d \boldsymbol{\kappa}
\end{array}\right.
$$

where $\boldsymbol{\kappa}$ is the wavenumber vector. Fig. (3.3) illustrates the representation of wavenumber components on a Fourier space. The interested reader may also refer to Schwartz and Huet [129] and Monin et al. [128] for a comprehensive understanding. For $i=j$ and $\boldsymbol{r}=0$ :

$$
\mathrm{R}_{i i}(0)=\left\langle u_{i}^{\prime} u_{i}^{\prime}\right\rangle=\int \phi_{i i}(\boldsymbol{\kappa}) d \boldsymbol{\kappa} .
$$

The turbulent kinetic energy $k$ is related to the correlation function and the spectral tensor of the velocity as

$$
k=\frac{1}{2} \overline{\boldsymbol{u}^{\prime 2}}=\frac{\overline{u_{1}^{\prime 2}}+\overline{u_{2}^{\prime 2}}+\overline{u_{3}^{\prime 2}}}{2}=\frac{1}{2} \mathrm{R}_{i i}(0)=\frac{1}{2} \int \phi_{i i}(\boldsymbol{\kappa}) d(\boldsymbol{\kappa}),
$$

\footnotetext{
${ }^{5}$ correlation means the tendency for two values or variables to change together, in either the same or opposite way.
} 
The energy spectrum function $E(\kappa)$ is then obtained from $\phi_{i j}(\boldsymbol{\kappa})$ by removing all the information about the direction of the velocity by considering the trace, i.e., $1 / 2 \phi_{i i}(\boldsymbol{\kappa})$. The information about the direction of the Fourier modes is removed by integrating over all wavenumbers. This is expressed as

$$
E(\kappa)=\frac{1}{2} \oint \phi_{i i}(\kappa) d \mathcal{S}(\kappa)
$$

where $\mathcal{S}(\kappa)$ is a sphere of radius $\kappa$ centred at the origin in Fourier space, as shown in Fig. $(3.3)$ $[130,122]$.

In isotropic turbulence, the spectral tensor $\phi_{i j}(\boldsymbol{\kappa})$ can be inferred from the second-order isotropic tensor which leads to its representation in spectral space as

$$
\phi_{i j}(\boldsymbol{\kappa})=\alpha(\kappa) \kappa_{i} \kappa_{j}+\beta(\kappa) \delta_{i j},
$$

where $\alpha(\kappa)$ and $\beta(\kappa)$ are scalar functions of $\kappa$. These scalar functions can be easily obtained 6 for incompressible isotropic turbulence to produce the spectral velocity tensor as

$$
\phi_{i j}(\boldsymbol{\kappa})=\frac{E(\kappa)}{4 \pi \kappa^{2}}\left(\delta_{i j}-\frac{\kappa_{i} \kappa_{j}}{\kappa^{2}}\right) .
$$

\subsubsection{One-dimensional spectra}

In experiments and numerical simulations, it is usually adequate to study the two-point correlation and energy spectra along a line, which is why a one-dimensional energy spectrum, $E_{11}^{(1)}(\kappa)$ where $\kappa \in\left(\kappa_{1}, \kappa_{2}\right.$ or $\left.\kappa_{3}\right)$, is normally used. Energy spectra for each velocity component can be calculated as

$$
E_{i j}^{(1)}\left(\kappa_{1}\right)=\iint \phi_{i j}(\boldsymbol{\kappa}) d \kappa_{2} d \kappa_{3}
$$

where the upper index (1) indicates the wavenumber component which stays after integration. For isotropic turbulence,

$$
E_{11}^{(1)}\left(\kappa_{1}\right)=\frac{1}{2} \int_{\kappa_{1}}^{\infty} \frac{E(\kappa)}{\kappa}\left(1-\frac{\kappa_{1}^{2}}{\kappa^{2}}\right) d \kappa
$$

$E_{22}^{(1)}\left(\kappa_{1}\right)$ can be determined using $E_{11}^{(1)}\left(\kappa_{1}\right)$ as

$$
E_{22}^{(1)}\left(\kappa_{1}\right)=\frac{1}{2}\left(E_{11}^{(1)}\left(\kappa_{1}\right)-\kappa_{1} \frac{d E_{11}^{(1)}\left(\kappa_{1}\right)}{d \kappa_{1}}\right)
$$

which can further be expressed in terms of $E(\kappa)$ as

$$
E_{22}^{(1)}\left(\kappa_{1}\right)=E_{33}^{(1)}\left(\kappa_{1}\right)=\frac{1}{4} \int_{\kappa_{1}}^{\infty} \frac{E(\kappa)}{\kappa}\left(1+\frac{\kappa_{1}^{2}}{\kappa^{2}}\right) d \kappa .
$$

\footnotetext{
${ }^{6}$ interested readers may refer to Pope [130] for a detailed derivation of the scalars
} 


\subsection{Two-dimensional turbulence}

A two-dimensional turbulent flow field has $u_{i}^{\prime} \neq 0$ for $i=1,2$, and the third component $u_{3}^{\prime}$ is always zero. In the case of the vorticity vector, all the components are zero except $\omega_{3}$. The velocity spectral tensor $\phi_{i j}$ remains in the same form as in Eq. (3.19), but the indexes $i$ and $j$ take only 1 or 2 as values. Similarly, the wavenumbers has only two components $\kappa_{1}$ and $\kappa_{2}$. However, the expression of $\phi_{i j}$ is now modified because the turbulent kinetic energy depends on two components of the velocity vector and on other hand, the integration surface in $\boldsymbol{\kappa}$ space is reduced to the circle of radius $\kappa$. Hence, the spectral velocity tensor for a two-dimensional turbulence is give by

$$
\phi_{i j}(\kappa)=\frac{E^{2 D}(\kappa)}{\pi \kappa}\left(\delta_{i j}-\frac{\kappa_{i} \kappa_{j}}{\kappa^{2}}\right) .
$$

The one-dimensional spectrum are also modified further and are expressed as

$$
\begin{aligned}
& E_{11}^{2 D(1)}\left(\kappa_{1}\right)=\frac{2}{\pi} \int_{\kappa_{1}}^{\infty} \frac{E^{2 D}(\kappa)}{\kappa^{2}} \sqrt{\kappa^{2}-\kappa_{1}^{2}} d \kappa, \\
& E_{22}^{2 D(1)}\left(\kappa_{1}\right)=\frac{2}{\pi} \int_{\kappa_{1}}^{\infty} \frac{E^{2 D}(\kappa)}{\kappa^{2}} \frac{\kappa_{1}^{2}}{\sqrt{\kappa^{2}-\kappa_{1}^{2}}} d \kappa .
\end{aligned}
$$

For two-dimensional turbulence, the longitudinal velocity spectrum function $E_{11}^{2 D(1)}\left(\kappa_{1}\right)$ is identical to the three-dimensional $E_{11}^{(1)}\left(\kappa_{1}\right)$, as well as the longitudinal correlation functions are identical. The two spectra are related to each other as

$$
E_{22}^{2 D(1)}\left(\kappa_{1}\right)=-\kappa_{1} \frac{d E_{11}^{(1)}\left(\kappa_{1}\right)}{d \kappa_{1}}
$$

\subsubsection{Liepmann spectrum}

Liepmann spectrum provides a better description of the energy-containing range [53]. In two dimensions, the turbulence spectra are defined by

$$
E_{l p}^{2 D}(\kappa)=\frac{16}{3 \pi} u_{r m s}^{\prime 2} \lambda^{5} \frac{\kappa^{4}}{\left(1+\lambda^{2} \kappa^{2}\right)^{3}}
$$

The one-dimensional spectrum for Liepmann spectra are given by

$$
\begin{aligned}
& E_{11}^{2 D_{l p}(1)}\left(\kappa_{1}\right)=\frac{u_{r m s}^{\prime 2} \lambda}{\pi} \frac{1}{\left(1+\lambda^{2} \kappa_{1}^{2}\right)} \\
& E_{22}^{2 D_{l p}(1)}\left(\kappa_{1}\right)=\frac{u_{r m s}^{\prime 2} \lambda}{\pi} \frac{2 \lambda^{2} \kappa_{1}^{2}}{\left(1+\lambda^{2} \kappa_{1}^{2}\right)^{2}}
\end{aligned}
$$

\subsection{2 von Kármán spectrum}

In 1948, von Kármán [131] proposed an expression for a function $E(\kappa)$ for atmospheric turbulence. The von Kármán spectrum provides a good description of both energy containing 


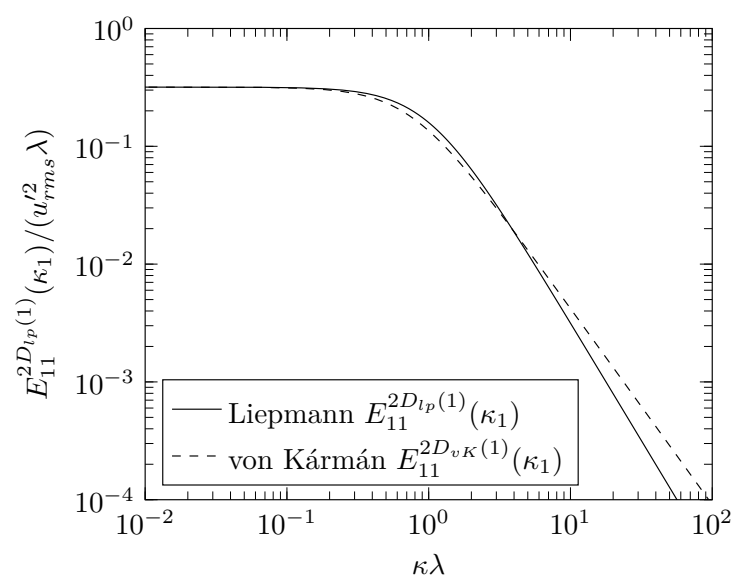

(a) $E(\kappa)_{(11)}^{2 D(1)}$ for Liepmann and von Kármán spectra

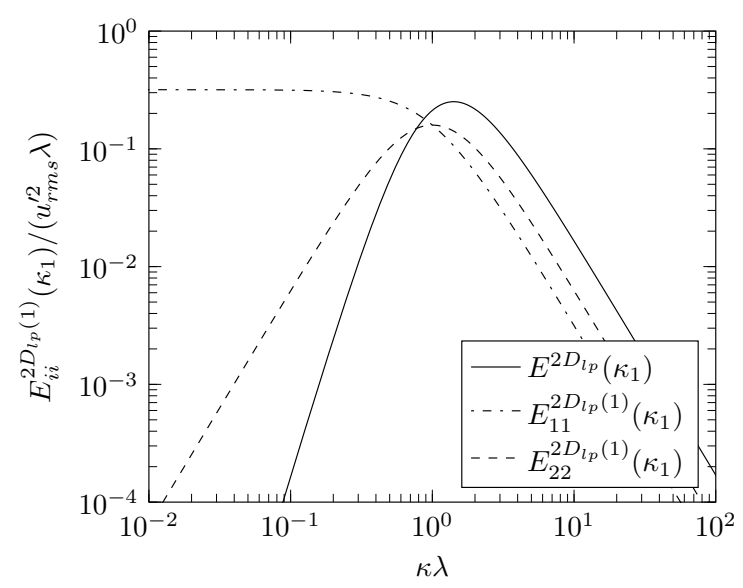

(c) Liepmann spectra for two-dimensional turbulence

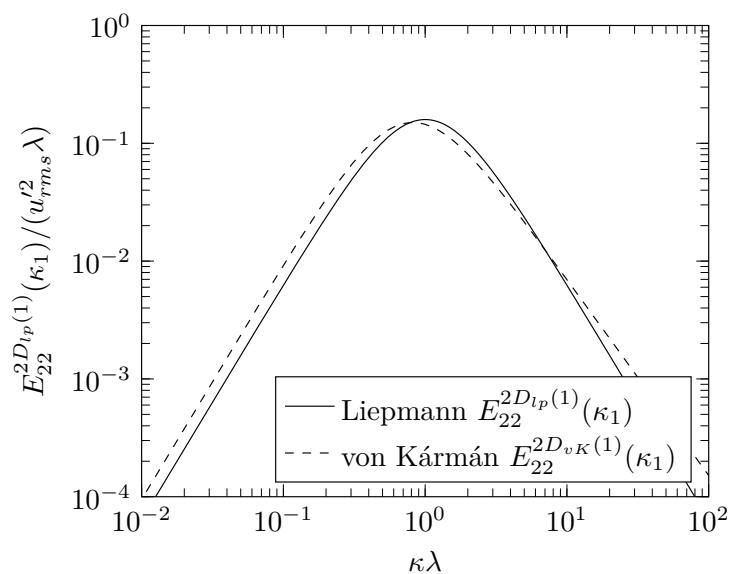

(b) $E(\kappa)_{(22)}^{2 D(1)}$ for Liepmann and von Kármán spectra

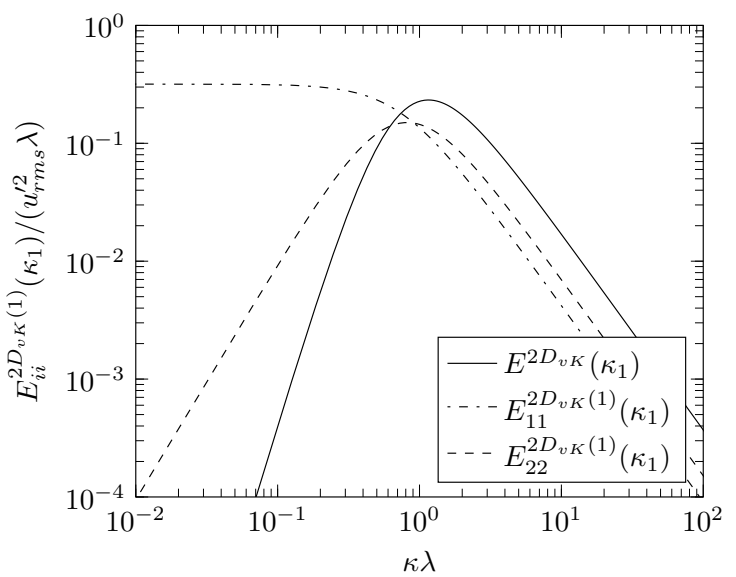

(d) von Kármán spectra for two-dimensional turbulence

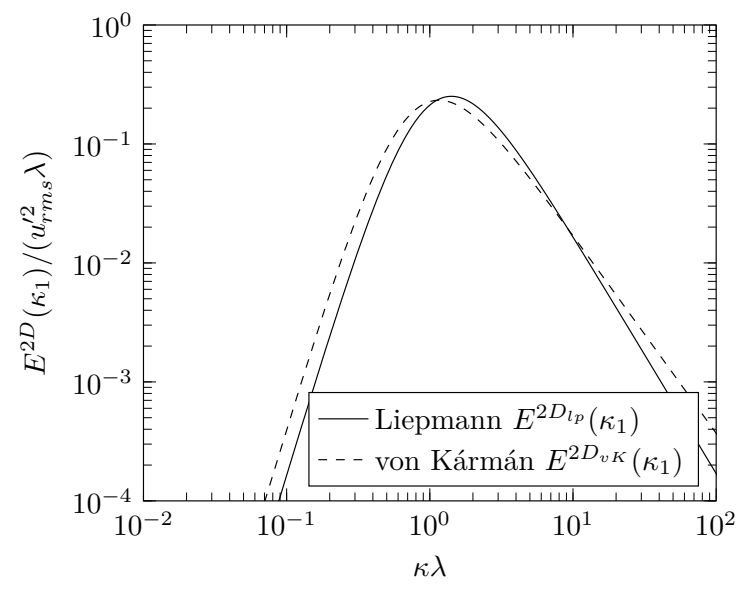

(e) Liepmann and von kármán spectra for twodimensional turbulence

Figure 3.4: One-dimensional spectra for longitudinal, $E(\kappa)_{(11)}^{2 D(1)}$, and transverse, $E(\kappa)_{(22)}^{2 D(1)}$, component of the Liepmann and von Kármán spectra for a two-dimensional turbulence 
range and the inertial sub-range. The modified function for two-dimensional turbulence reads as

$$
E_{v K}^{2 D}(\kappa)=\frac{110}{27 \pi} u_{r m s}^{\prime 2} \lambda \frac{\hat{\kappa}^{4}}{\left(1+\hat{\kappa}^{2}\right)^{17 / 6}}
$$

with

$$
\hat{\kappa}=\kappa / \zeta, \quad \zeta=\frac{\Gamma(1 / 2) \Gamma(5 / 6)}{\lambda \Gamma(1 / 3)},
$$

where $u_{r m s}^{\prime 2}$ is nothing else but $\overline{u^{\prime 2}}$ given in Eq. (3.3) related to the mean flow velocity $\overline{\mathrm{u}}$ by $u_{r m s}^{\prime 2}=\left(T u \cdot \overline{u^{\prime}}\right)^{2} \cdot \lambda$ is the integral length scale, and $\hat{\kappa}$ is the reduced wavenumber, and $\Gamma$ is the gamma function. The longitudinal and transverse spectrum for the energy spectra are given as

$$
\begin{gathered}
E_{11}^{2 D_{v K}(1)}\left(\kappa_{1}\right)=\frac{u_{r m s}^{\prime 2} \lambda}{\pi} \frac{1}{\left(1+\hat{\kappa}_{1}^{2}\right)^{5 / 6}}, \\
E_{22}^{2 D_{v K}(1)}\left(\kappa_{1}\right)=\frac{5 u_{r m s}^{\prime 2} \lambda}{3 \pi} \frac{\hat{\kappa}_{1}^{2}}{\left(1+\hat{\kappa}_{1}^{2}\right)^{11 / 6}} .
\end{gathered}
$$

Fig. (3.4) shows the spectra for both Liepmann and von Kármán descriptions of turbulence in two-dimensions.

\subsection{Representation of a Turbulent Velocity Field Using Vortex Particles}

The foundation of this work rests upon the Helmholtz-decomposition used in vortex methods to approximate vorticity using a set of vortices. Eq. (2.17) depicts the velocity-field of the entire flow-field through a single streamfunction. This single streamfunction unites the three main streamfunctions of the flow. In this section, the streamfunction that introduces the disturbance to the flow is formulated, which is then used to derive a given input velocity field using vortex particles. Introduction of disturbances in VPM is commonly called turbulence modelling. Recently, Tolba and Morgenthal [96] reviewed a few influential research studies $\underline{[109}$, $\underline{110}, 111$ in this field. Furthermore, chapter 1 reviews the research on successfully creating inflow turbulence using discrete particles in aeroacoustics, primarily for airfoil turbulence interaction noise, suggesting that these methods had some drawbacks such as the absence of stochastic nature of turbulence and inability to fall short on reducing the high computational costs involved. In the framework of proposed method, upcoming sections introduce to a new technique, which is based on the statistical study of vortex particles, to realise atmospheric turbulence. In this study, Liepmann and von Kármán spectra for two-dimensional turbulence are used as a reference for the realisation of the turbulent inflow.

\subsubsection{Two-dimensional system: Point vortex}

The velocity and vorticity formulation of Euler equations for a two-dimensional incompressible vortical flow is given in Eqs. (2.9)-(2.13). The relationship between a vorticity source and 


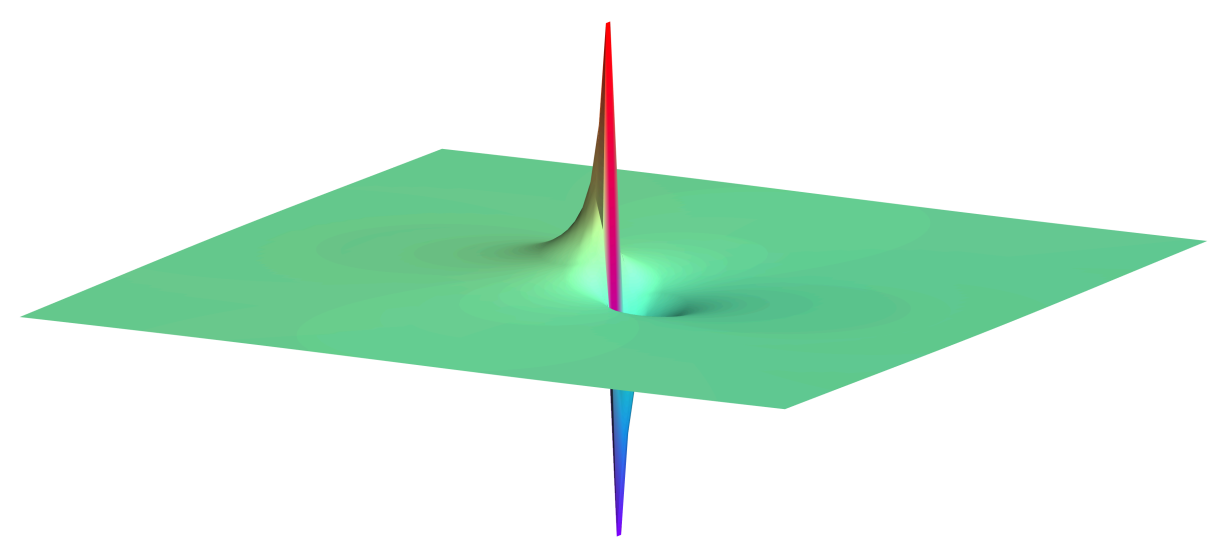

(a) u, horizontal induced velocity by a point vortex

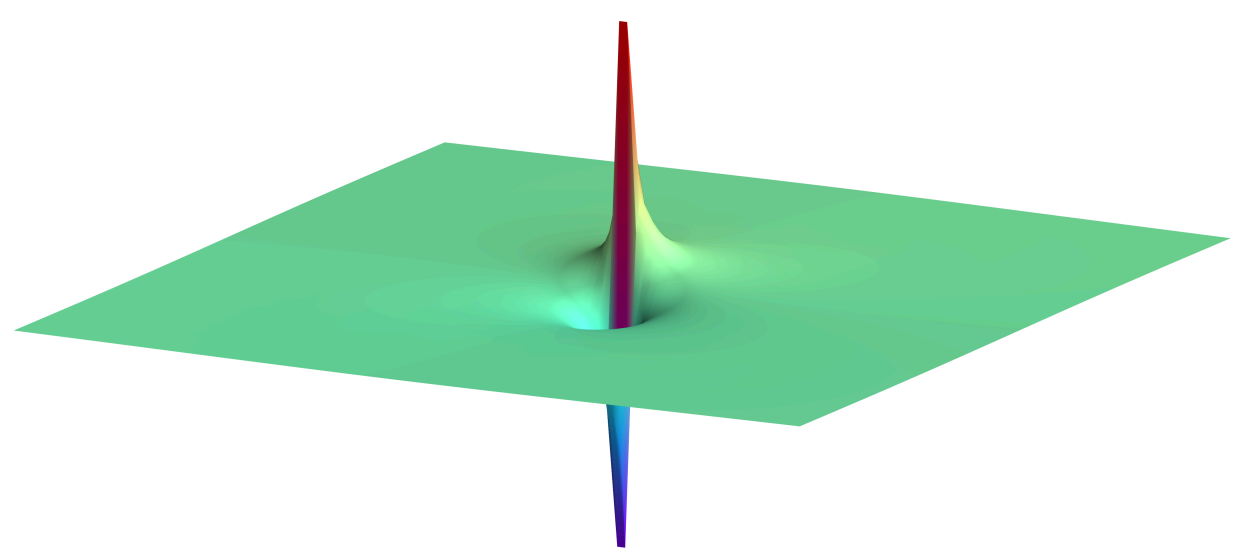

(b) v, vertical induced velocity by a point vortex

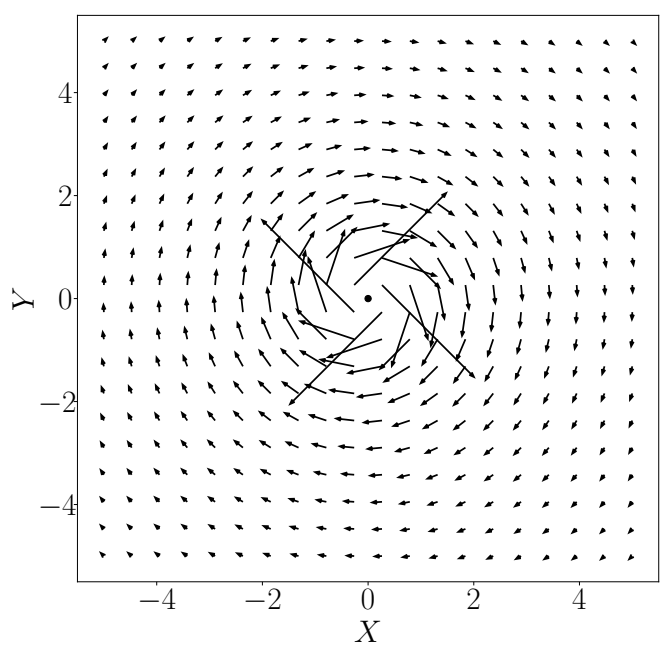

(c) streamfunction showing the direction of rotation of fluid volume around a point vortex

Figure 3.5: Flow field: velocity field plotted as $z$-axis for a point vortex (a) $u_{1}$, (b) $u_{2}$; and (c) two-dimensional plot of the velocity field on $x y$-plane 
the induced streamfunction and velocity in an infinite plane with no boundaries uses the Green's function approach. Here, the vorticity source is concentrated at points and has infinite strength in a pointwise sense, but finite strength in an area-averaged sense. An expression for the streamfunction equation can be obtained for the particular case where there is a single "impulse" vorticity in an infinite domain as

$$
\nabla^{2} \psi=-\omega \frac{\delta(r)}{2 \pi r}, \mathbf{r}=\mathbf{x}-\mathbf{x}^{\prime}, r=\left|\mathbf{x}-\mathbf{x}^{\prime}\right|,
$$

where $\omega$ is clearly the strength of a concentrated vortex point. The Green's function solution is then

$$
\psi(\mathbf{x})=-\omega\left(\mathbf{x}^{\prime}\right) \frac{\ln r}{2 \pi}=-\omega\left(\mathbf{x}_{\mathbf{0}}\right) \frac{\ln \left|\mathbf{x}-\mathbf{x}^{\prime}\right|}{2 \pi},
$$

From Eq. (3.37), the velocity associated with this streamfunction field is calculated as

$$
u=\nabla \times(\psi \mathbf{k})=-\omega\left(\mathbf{x}^{\prime}\right) \frac{\nabla \times(r \mathbf{k})}{2 \pi r}=-\omega\left(\mathbf{x}^{\prime}\right) \frac{\nabla r \times \mathbf{k}}{2 \pi r},
$$

where

$$
r=\sqrt{\left(x-x^{\prime}\right)^{2}+\left(y-y^{\prime}\right)^{2}}, \nabla r=\mathbf{r} / r .
$$

Hence, the velocity induced by a point vortex with strength $\omega$ is then given as

$$
\mathbf{u}(\mathbf{x})=-\omega\left(\mathbf{x}^{\prime}\right) \frac{\mathbf{r} \times \mathbf{k}}{2 \pi r^{2}}=-\omega\left(\mathbf{x}^{\prime}\right) \frac{\left.\left(\mathbf{x}-\mathbf{x}^{\prime}\right)\right) \times \mathbf{k}}{2 \pi\left|\left(\mathbf{x}-\mathbf{x}^{\prime}\right)\right|^{2}} .
$$

Fig. (3.5a) shows the horizontal velocity induced by a point vortex in three-dimensional view where the magnitude of the velocity, $u$, is shown on the $z$-axis. Similarly, Fig. (3.5b) shows the vertical component of the velocity. In both figures, even though the spikes are plotted to a finite length (because of space constraints), but their main purpose is to show that when a point approaches the centre of a point vortex, it begins to feel the imperceptible velocity that becomes infinite at the absolute centre. Fig. $(\underline{3.5 \mathrm{c})}$ shows the streamfunction field induced by a point vortex.

\subsubsection{Multiple point vortices}

One major advantage of working with the above equations is their linearity which makes it possible to invoke the principle of superposition to account for the presence of multiple point vortices in the domain. The expressions for the streamfunction and velocity expressions imparted by each of the point vortices in a domain with multiple point vortices can be written as

$$
\begin{gathered}
\psi_{N}(\mathbf{x})=-\omega\left(\mathbf{x}_{N}^{\prime}\right) \frac{\ln \left|\mathbf{x}-\mathbf{x}_{N}{ }_{N}\right|}{2 \pi}, \\
\mathbf{u}_{N}(\mathbf{x})=-\omega\left(\mathbf{x}_{N}^{\prime}\right) \frac{\left(\mathbf{x}-\mathbf{x}_{N}^{\prime}\right) \times \mathbf{k}}{2 \pi\left|\mathbf{x}-\mathbf{x}_{N}\right|^{2}},
\end{gathered}
$$




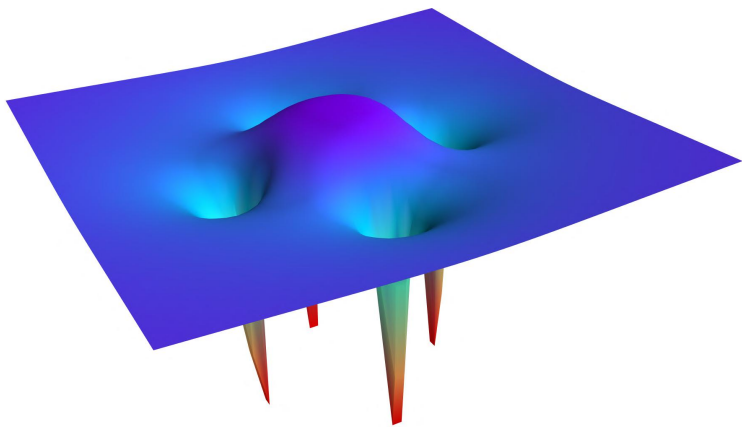

(a) flow field: velocity field plotted as $z$-axis for all vortices with equal directional strength (clock-wise)

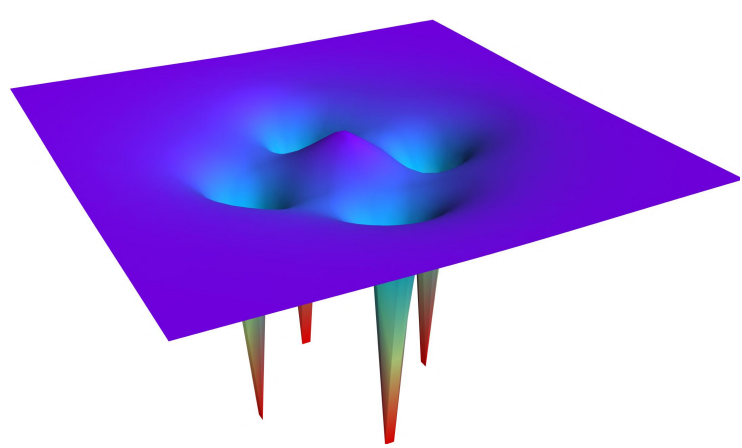

(b) flow field: velocity field plotted as $z$-axis for vortices arranged in alternate directional strength

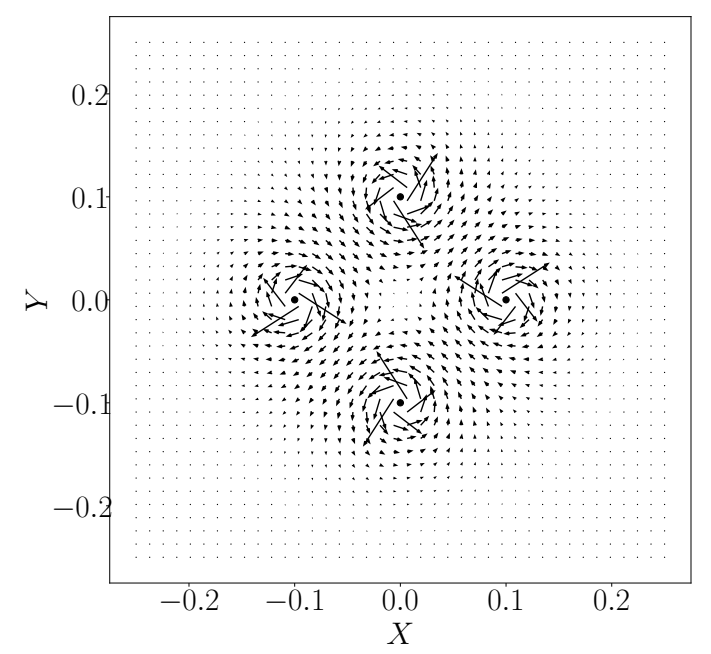

(d) flow field: velocity field plotted for vortices arranged in alternate directional strength (c) flow field: velocity field plotted for all vortices with equal directional strength (clock-wise)

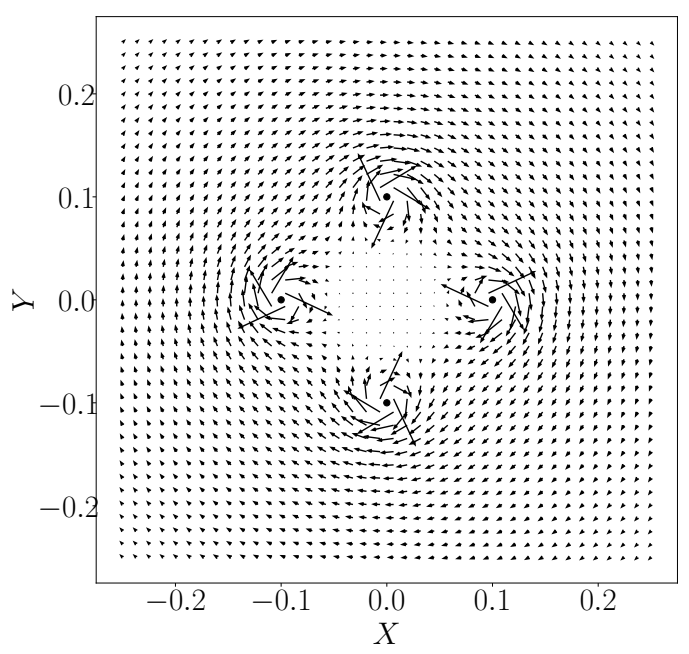

Figure 3.6: Flow field induced by four point vortices located at $( \pm 0.1,0)$ and $(0, \pm 0.1)$

where $\mathbf{x}$ is the point where the vortex influence is calculated, $\mathbf{x}_{N}^{\prime}$ is the location of the $N$-th vortex and $\omega\left(\mathbf{x}_{N}\right)$ is its strength.

Fig. (3.6a) and $(\underline{3.6 \mathrm{~b}})$ shows the velocity field due to four vortices placed at $( \pm 0.1,0)$ and $(0, \pm 0.1)$ arranged with same directional strength and alternate directional strength, respectively, in a Cartesian coordinate system. There exists a problem when approximating the vorticity with delta functions (point vortices), i.e. infinite spikes, which is an associated singularity in the streamfunction and velocity field. This singularity appears when the distance to the point vortex becomes very small, $\left|\mathbf{x}-\mathbf{x}^{\prime}{ }_{N}\right| \rightarrow 0$ : both streamfunction and velocity become very large; the first blows up logarithmically fast and the other like $\mathbf{x}^{-1}$. This is quite problematic in the evaluation of the sums. This singular behaviour is shown for vortices rotating in same direction in Fig. (3.6a) and (3.6c). The area in the middle exhibits less or almost null rotation, with weaker rotation region between adjacent pairs. Fig. (3.6b) and (3.6d) shows a strong but smaller singular region around origin for counter-rotating vortices, with stronger rotation region between the pairs of opposite-signed vorticity. This behaviour was fundamentally expected because when two vortices rotating in the opposite direction come close, the resultant 
velocity tends to high values which are non-physical in nature. Whereas when two vortices are rotating in the same direction, the resultant velocity tends to superficial values, close to zero (e.g. a dipole).

Eq. (3.42) can be modified for an infinite number of vortex particles as

$$
u=\frac{1}{2 \pi} \iint \mathbf{K}\left(\mathbf{x}-\mathbf{x}^{\prime}\right) \omega\left(\mathbf{x}^{\prime}\right) d \mathbf{x}^{\prime} .
$$

The above equation is famously known as Biot-Savart law. The first term inside the integral is the kernel, $\mathbf{K}$, which can be interpreted as the velocity generated by a point vortex, and the entire integral expresses the effects of all the vortices added linearly. From the discussion so far, it is clear that if the vorticity consists of particles carrying a certain amount of vorticity as

$$
\omega\left(\mathbf{x}_{N}\right)=\Gamma_{N} \delta\left(\left|\mathbf{x}-\mathbf{x}_{N}^{\prime}\right|\right) \Rightarrow \delta\left(\left|\mathbf{x}-\mathbf{x}_{N}^{\prime}\right|\right) \rightarrow 0 \quad \forall\left|\mathbf{x}-\mathbf{x}_{N}^{\prime}\right| \rightarrow \infty
$$

where $\Gamma_{N}$ is the circulation around the $N$-th particle, besides it is necessarily required that $\delta\left(\mathbf{x}-\mathbf{x}_{N}^{\prime}\right)$ decays quickly with distance from the centre of the vortex.

\subsubsection{Kernel mollification}

The singularity of the kernel $\mathbf{K}$ can lead to high nonphysical values of the vorticity field $\omega$ for a case where two particles approach each other when implementing directly with Eq. (3.43). The solution to this problem is eliminating the singularity associated with $\mathbf{K}$ by either using a cut-off radius which converts $\mathbf{K}$ into a mollified $\mathbf{K}_{\varepsilon}[83]$ or by using a viscous vortex model with a finite core size [132] by multiplying a factor to remove the singularity. Abedi [133] reviewed the application of few relevant viscous vortex models, Rankine [134], Scully et al. [135], Vatistas [136], and Bagai and Leishman [137] in his study on vortex filaments.

In this study, the mollification of $\mathbf{K}$ is achieved by its convolution with a cut-off function $\chi$. The accuracy of the approach which allows a precise control relies on the replacement of $\mathbf{K}$ by $\mathbf{K}_{\varepsilon}$ which is given by

$$
\mathbf{K}_{\varepsilon}=\mathbf{K} \star \chi_{\varepsilon},
$$

where $\mathbf{K}_{\varepsilon}$ is the smoothed velocity kernel and $\chi$ is a smooth cut-off function which satisfies $\int \chi(\mathbf{x}) d \mathbf{x}=1$. Eq. (3.45) further modifies Eq. (2.15) and (2.16) into

$$
\begin{gathered}
\mathbf{u}^{h}=\mathbf{K}_{\varepsilon} \star \omega^{h}, \\
\omega_{\varepsilon}^{h}(\mathbf{x})=\sum_{p} \Gamma_{p} \chi_{\varepsilon}\left(\mathbf{x}-\mathbf{x}_{\mathbf{p}}^{\mathbf{h}}\right),
\end{gathered}
$$

where $\Gamma_{p}$ is an estimate of the initial circulation around the particle, $\chi_{\varepsilon}$ is a cut-off function or blob function scaled by the core size $\varepsilon$. The main distinction between vortex methods and grid-based methods lies in the treatment of vorticity transport equations. In vortex methods, a set of discrete particles are used to approximate initial circulation present in the domain whereas, in grid-based methods, the governing equations are projected on a finite-dimensional space. The fact that smooth velocity kernels help in dropping the infinite spikes in the vorticity 
and velocity field, on the other hand, since the vortex methods are solved on moving grids, thus making them highly sensitive to the smoothness of the velocity field. Hald [102, 138$]$ and Beale $[\underline{78}, 139]$ conducted studies on the convergence of vortex method based on cut-off functions and showed that the method is sensitive to velocity smoothing which makes it prone to errors. A necessary constraint [140] for convergence is particles overlap; i.e., the ratio of the blob radius to the particle spacing, must be greater than unity. Interested readers are referred to Cottet and Koumoutsakos [83] for a complete understanding of the accuracy of cut-off functions or blobs.

Additionally, smoothness, order of accuracy, and computational cost are also the criteria for determining a cut-off function. Gaussian-based cut-offs are regularly preferred because of their smoothness and fast decay. However, they are expensive to compute, which is why in this work, their influences are calculated in advance and stored in a/an matrix/array employing the linearity of the system, thus the application of superposition principle (discussed later in next chapter). In two dimensions, the Gaussian cut-off function is given by

$$
\chi(r)=\frac{1}{\pi} e^{-r^{2}}
$$

which yields a smoothed velocity mollified Gaussian kernel as

$$
\left.\mathbf{K}_{\varepsilon}(\mathbf{x})=\frac{\mathbf{x}}{2 \pi|\mathbf{x}|^{2}}\left[1-e^{|\mathbf{x}|^{2} \varepsilon^{2}}\right)\right]
$$

In two-dimensions, the velocity field is reconstructed from the vorticity field by the application of Biot-Savart law. From the definition of $\mathbf{K}=\nabla G$, Eq. (3.43) can be expanded for the fluctuating velocity field, $\mathbf{u}^{\prime}$ as

$$
\mathbf{u}^{\prime}(\mathrm{x}, t)=\nabla^{\perp} \underbrace{\int G\left(\mathrm{x}, \mathrm{x}^{\prime}\right) \omega\left(\mathrm{x}^{\prime}, t\right) d \mathrm{x}^{\prime}}_{\psi_{\text {turb }}},
$$

where $G$ is a spatial Green's function filter, $\omega=\left(\omega_{1}, \omega_{2}, \omega_{3}\right)$ is the white noise field which is reduced to a single term, $\omega=\left(0,0, \omega_{3}\right)$, for two-dimensional turbulent flow.

Vortex particles with mollified cores are widely used to generate initial turbulent conditions in Large-Eddy Simulations. This is called Synthetic Eddy Modelling (SEM) in grid-based methods. Jarrin et al. [141] introduced a method based on the classical view of turbulence as a superposition of coherent structures. The method was capable of generating prescribed first and second-order one-point statistics, characteristic length and time scales, and the shape of coherent structures. For CAA applications, the divergence-free condition is crucial to prevent artificial artefact noise that may arise from the synthetically generated turbulence. Sescu and Hixon [142] observed that the convection velocity of synthetically generated turbulence need be synchronised with the local mean-flow velocity in order to assure a fair aeroacoustic environment not intervening with genuine sound waves. They proposed the concept of shape function defined by a vector potential (scalar potential in two-dimensions) to resemble the shape of an eddy, a concept very similar to the concept of mollification of the velocity kernel using cut-off functions. The functions proposed are any differentiable function, with compact 
support of its first derivative, e.g. Gaussian shape function, Mexican-hat function_ ${ }^{7}$ and Morlet mother. To build these representations, it is thus assumed that each of mollified vortices is associated with a localised shape function, $\psi=f(r)$ that is radially symmetric about the centre of the vortex, that decays as we move away from the centre, and whose integral over the entire plane is unity. A general form of the scalar potential, $\psi_{\text {turb }}$, for such a space can be written as:

$$
\psi_{\text {turb }}(r, t)=\psi_{n}\left(\frac{\left|r-r^{\prime}\right|}{\lambda}, \frac{t-t^{\prime}}{t_{\lambda}}\right)
$$

$\psi_{n}$ represents a dimensionless shape function for each individual vortex. $r^{\prime}$ is the spatial and $t^{\prime}$ is the temporal location of the vortex. $\lambda$ and $t_{\lambda}$ denote the turbulent length and time scales, respectively. For the current study, the shape functions considered for the vortices are the Gaussian wave and Mexican-hat which are given by

$$
\begin{gathered}
\psi_{G}(\boldsymbol{r}, t)=\gamma \varrho^{-\frac{1}{2}} e^{-(3 \varrho \boldsymbol{r}))^{2}}, \quad(\text { Gaussian }) \\
\psi_{M h}(\boldsymbol{r}, t)=\gamma \varrho^{-\frac{1}{2}}\left[1-(4 \varrho \boldsymbol{r})^{2}\right] e^{-(\sqrt{8} \varrho r)^{2}}, \quad \text { (Mexican-hat) }
\end{gathered}
$$

respectively. The right-hand side is a transcendental function, called the shape function (a spatiotemporal polynomial), with $\gamma$ as the directional strength of the vortex, $\varrho$ controls the size of the vortex (radius of the vortex core, $R=1 / \sqrt{(} \varrho), r$ is the position of the vortex in the computational domain. Sescu [142] also showed that Eqs. (‥51-3.53) satisfy the Euler equations and therefore genuinely remove the possibility of causing pressure fluctuations from the synthetic vortices. Using Eqs. (3.52) and (3.53), the tangential velocity can be expressed as

$$
\begin{gathered}
u_{\theta}(\mathbf{x})=18 \gamma \mathbf{x} R^{-3} \exp \left(-9 \mathbf{x}^{2} / R^{4}\right), \\
u_{\theta}(\mathbf{x})=16 \gamma R^{-3} \exp \left(-8 R^{-4} \mathbf{x}^{2}\left(3-16 R^{-4} \mathbf{x}^{2}\right)\right) \mathbf{x} .
\end{gathered}
$$

Above equations are then employed to calculate and visualise the velocity field induced by the mollified vortex particles. The particles are stationed in a finite two-dimensional plane with their centres coinciding with the origin at $(0,0)$. Fig. $(\underline{3.7 \mathrm{a}})$ and $(\underline{3.7 \mathrm{c}})$ show the horizontal and vertical components of the velocity field due to a Gaussian vortex with $z$-axis plotted as the magnitude, Fig. $(3.7 \mathrm{~b})$ and $(\underline{3.7 \mathrm{~d}})$ show the same attributes of the velocity field due to a Mexican-hat vortex. The infinite spikes in the velocity field (singularity) of a point vortex as observed in Fig. (3.5a) and $(\underline{3.5 \mathrm{~b}})$ are mollified by using smooth kernels, as shown in Fig. (3.7). The velocity fields are calculated with identical directional strength for all the vortices, whereas, the radius of the Gaussian vortex and Mexican-hat vortex are kept equal throughout the analysis. Then, an observation point approaching the centre of a Gaussian particle experiences an increase in velocity until it reaches the core of the vortex, inflexion point, and then this increase quickly changes to a falling zero at the absolute centre. In the case of a Mexican-hat vortex, the observation point first experiences a sudden drop in the

\footnotetext{
${ }^{7}$ a real-valued wavelet, Mexican hat also known as Marr wavelet is the second derivative of a Gaussian [143]
} 


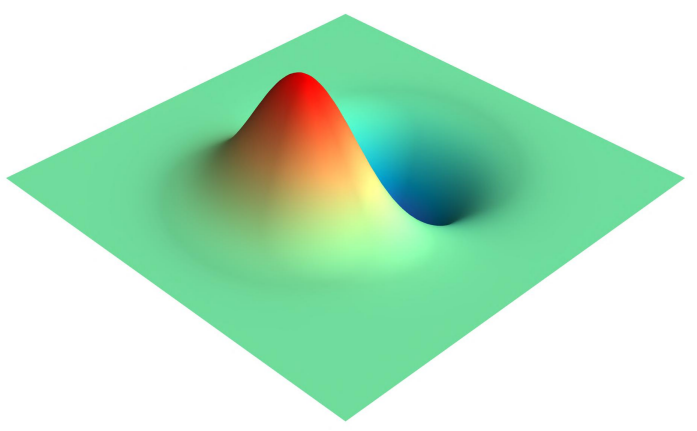

(a) $u_{1}$, horizontal induced velocity by a Gaussian waveform mollified vortex

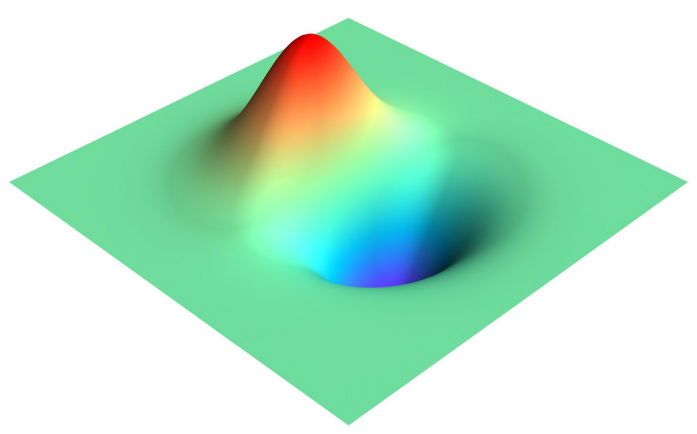

(c) $u_{2}$, vertical induced velocity by a Gaussian waveform mollified vortex

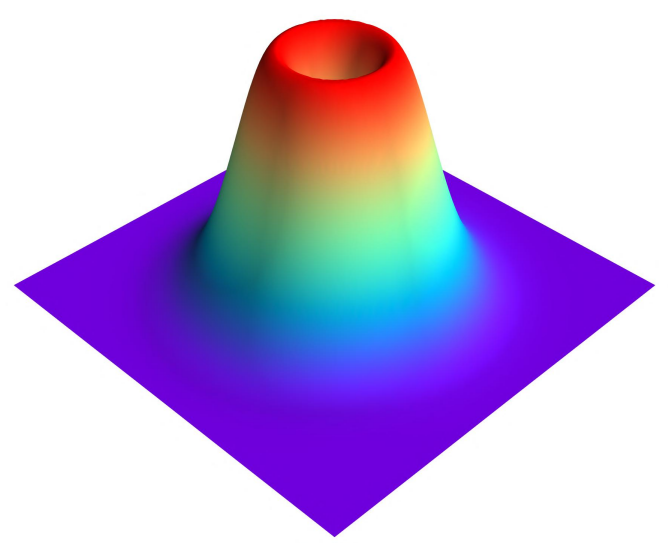

(e) total velocity induced by Gaussian waveform mollified vortex

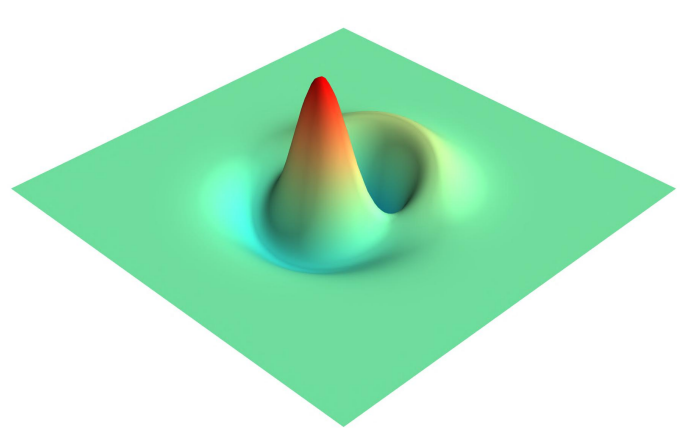

(b) $u_{1}$, horizontal induced velocity by a Mexican-hat waveform mollified vortex

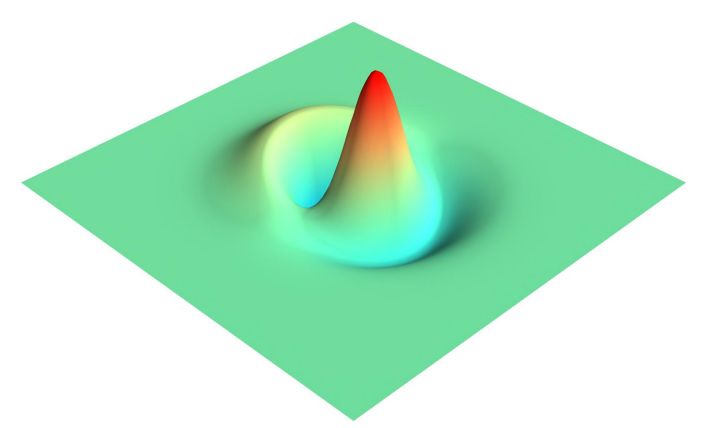

(d) $u_{2}$, vertical induced velocity by a Mexican-hat waveform mollified vortex

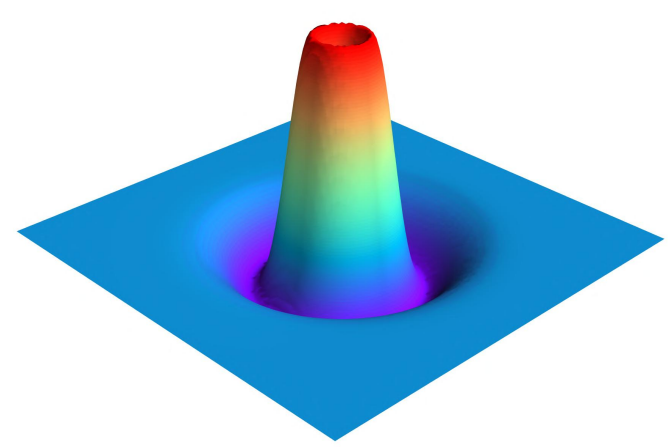

(f) total velocity induced by Mexican-hate waveform mollified vortex

Figure 3.7: Flow field: velocity field plotted as $z$-axis for a Gaussian (a, c, e) and Mexican-hat (b, d, f) waveform vortex (a) $u_{1}$, (b) $u_{2}$; and (c) $\mathrm{U}$ 


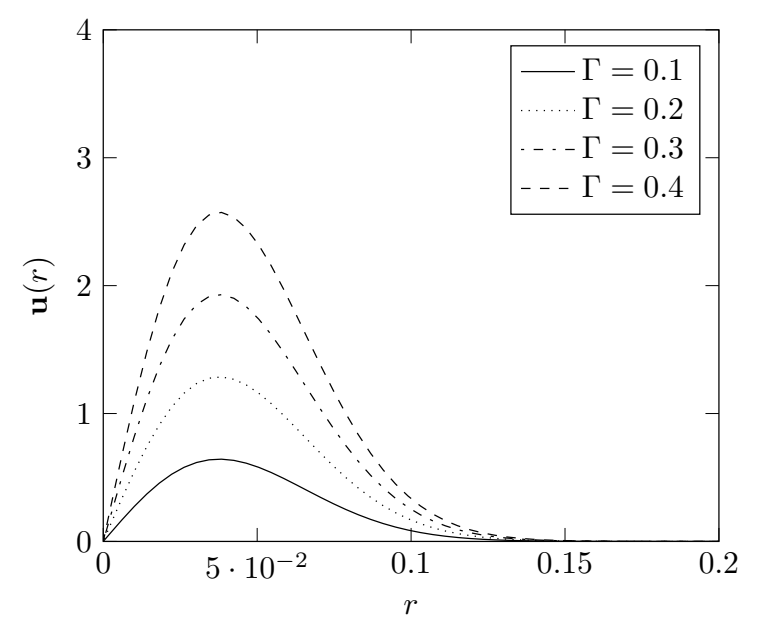

(a) Gaussian vortices with identical $R$ and different $\Gamma$

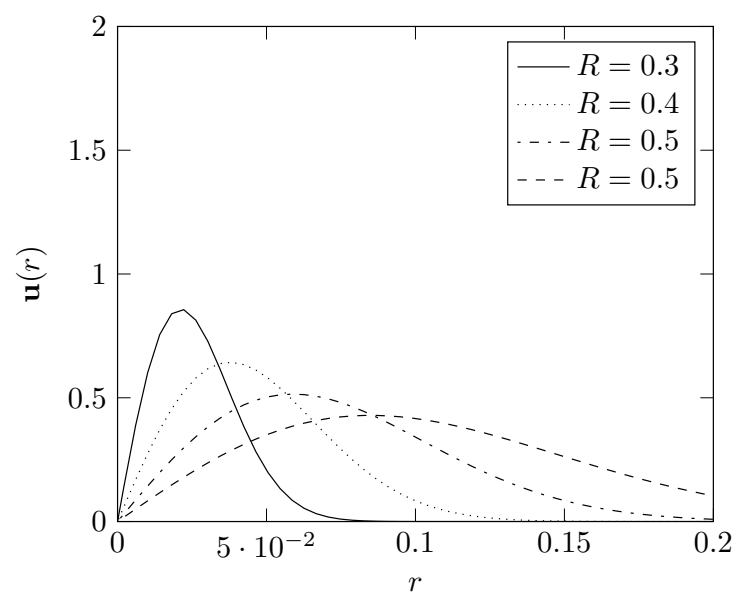

(c) Gaussian vortices with identical $\Gamma$ and different $R$

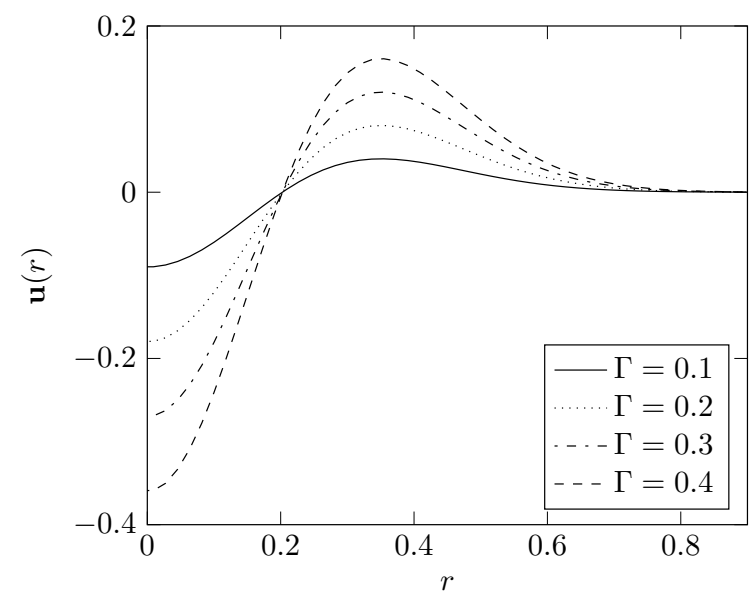

(b) Mexican-hat vortices with identical $R$ and different I

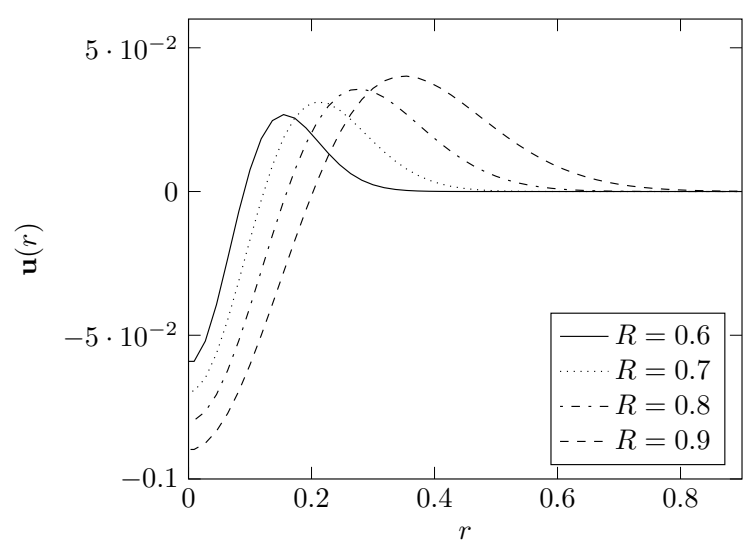

(d) Mexican-hat vortices with identical $\Gamma$ and different

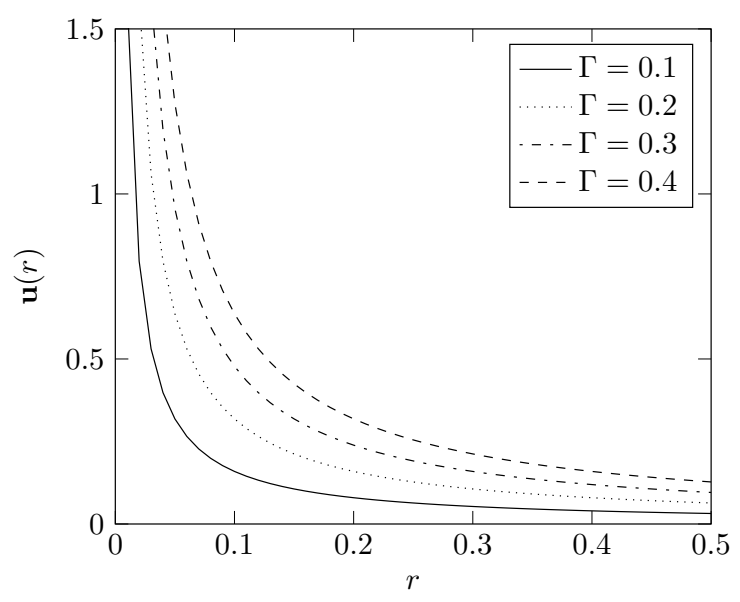

(e) velocity profile of point vortices

Figure 3.8: Velocity profiles of the Gaussian and the Mexican-hat mollified kernels based on the variations in their circulation strength and radius

induced velocity which then starts to increase quickly until the next inflexion point from where it drops to the minimum or zero value. 


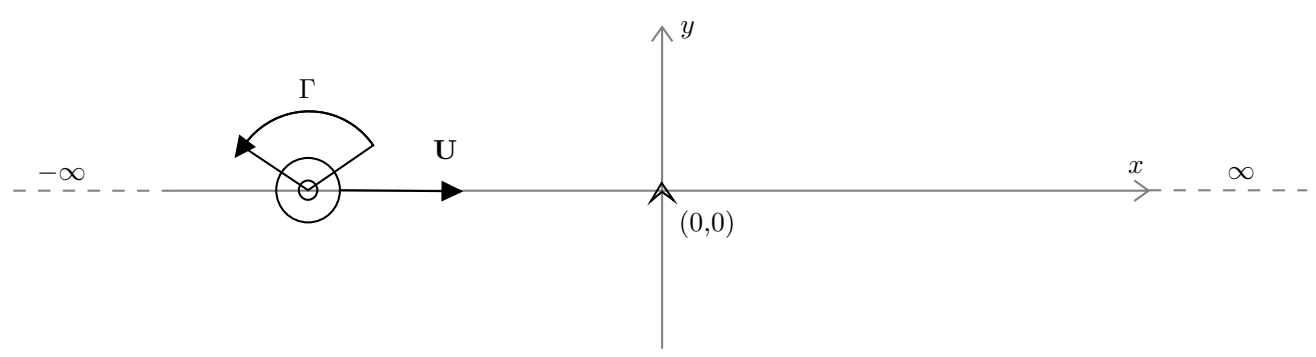

Figure 3.9: single vortex moving with the freestream velocity

The two main parameters which regulate the behaviour of the vortex particle are its strength and radius. Any physical velocity profile can be readily obtained by playing around with these two parameters on a set of discrete mollified vortex particles. The velocity magnitude is calculated for a range of $r$ which allows satisfying $\lim _{r \rightarrow \infty} u(r) \rightarrow 0$. Fig. (3.8) shows the profiles of the Gaussian and the Mexican-hat mollified kernel vortex given by Eq. (3.54) and $(\underline{3.55})$. From Fig. $(\underline{3.8 \mathrm{a})}$ and $(\underline{3.8 \mathrm{~b}})$, the linear dependence of the strength is clearly shown as the variations in the strength is reflected in the velocity profile by only a factor of scale. The radius, $R$, of the vortices, are kept constant at 0.1 with strength, $\Gamma$, varied. However, this is not the case with the radius. The plot in Fig. $(\underline{3.8 \mathrm{c})}$ and $(\underline{3.8 \mathrm{~d})}$ shows the nonlinear dependency of the radius on the velocity profiles. In these cases, strength, $\Gamma$, is kept constant at 0.4 with radius, $R$, varied. Fig. $(\underline{3.8 \mathrm{e})}$ shows the velocity profiles of a point vortex of different strengths. The induced velocity is evidently going to nonphysical values near the centre of the vortex.

\subsubsection{Single-particle statistics}

The reconstruction of a turbulent velocity field using a set of discrete vortices rests upon the ability to superimpose the disturbances associated with each particle, thus enabling the addition of all the disturbances utilising the superposition principle. The behaviour of each particle contributes to the overall turbulent velocity field, which is why it is crucial to investigate the statistical properties of these vortex particles. For a statistically stationary process [130], the autocorrelation of a single variable $u^{\prime}(t)$ at two times $t_{1}$ and $t_{2}$ is defined as

$$
\mathrm{R}\left(t_{1}, t_{2}\right)=\left\langle u^{\prime}\left(t_{1}\right) u^{\prime}\left(t_{2}\right)\right\rangle .
$$

In the case of a statistically stationary process, the autocorrelation depends only on the difference $\tau=t_{2}-t_{1}$ but not on the actual instants of time, $t_{1}$ and $t_{2}$. Thus Eq. (3.56) can be rewritten as

$$
\mathrm{R}(\tau)=\left\langle u^{\prime}(t) u^{\prime}(t+\tau)\right\rangle
$$

Above equation is then used to define Power Spectral Density (PSD) denoted by $S_{x x}$

$$
S_{x x}(f)=\int_{-\infty}^{\infty} \mathrm{R}(\tau) e^{-i 2 \pi f \tau} d \tau .
$$

A vortex particle travels far away from infinity to an observation point that exits at a point located at the origin in the Cartesian plane, as shown in Fig. (3.9). This particle induces a velocity field throughout its convection from one end to another. The time-history of the 


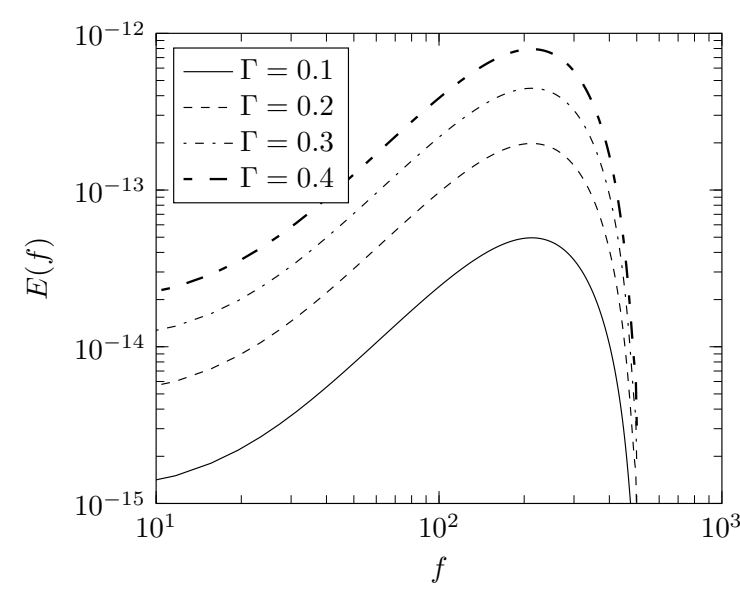

(a) Mexican-hat $R=0.1$

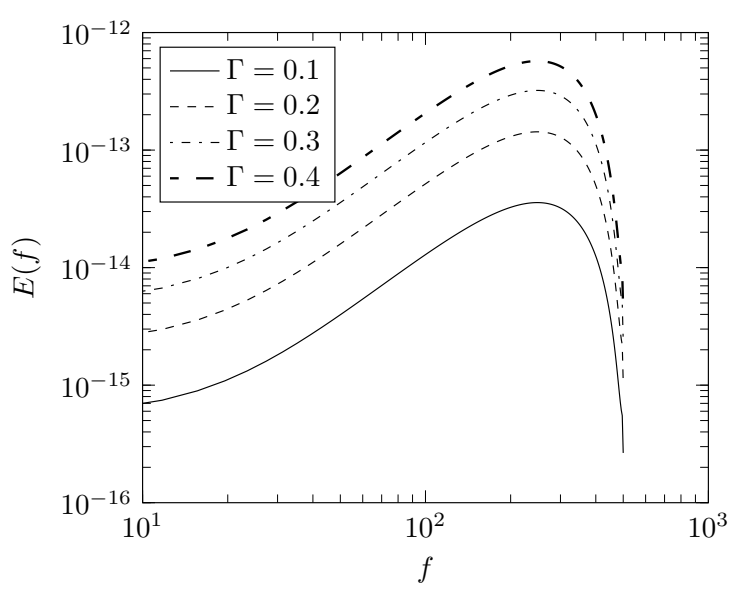

(c) Gaussian $R=0.1$

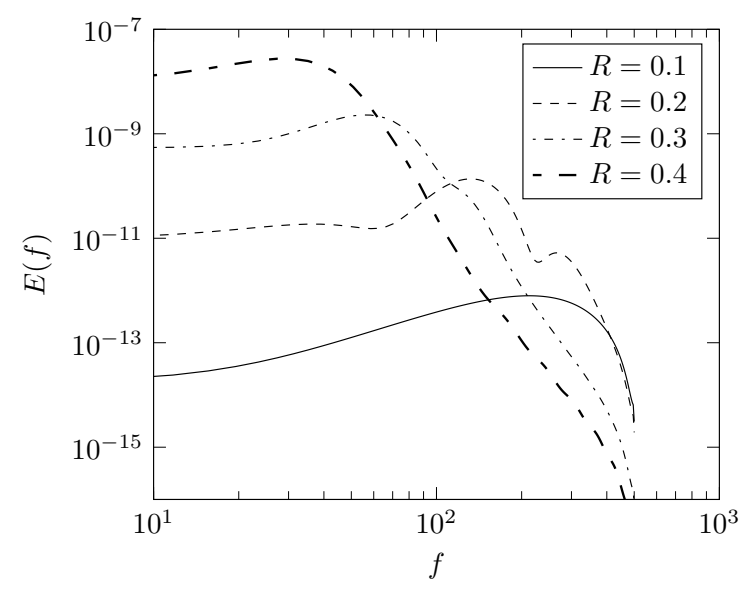

(b) Mexican-hat $\Gamma=0.4$

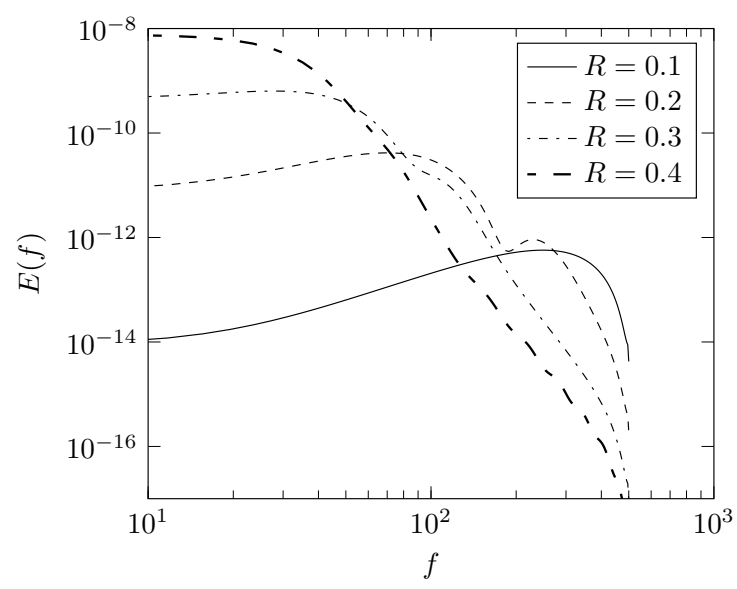

(d) Gaussian $\Gamma=0.4$

Figure 3.10: Power Spectral Density (PSD) due to Gaussian waveform vortices and Mexican-hat waveform vortices (Note: $E(f)=S_{x x}(f)$

fluctuating velocity, $u^{\prime}(t)$, experienced at the observation point is stored and is used to calculate PSD using Eqs. (3.57)-(3.58). The investigation is carried out for a Gaussian vortex particle and a Mexican-hat vortex particle for four different cases of strength and radius each. As can be seen in Fig. (3.10a) and Fig. (3.10c), the shape of the power spectrum remains the same for all the vortex strengths; however, the spectrum shifts upwards by a factor of a finite number while the strength is increased for both particles. From this, it can be said that a quantitative shift in the magnitude of vortex strength can be easily accounted by a corresponding vertical shift in the principal spectrum. The radius of the core of the vortex particles is fixed at 0.1 . Furthermore, unlike the shifts in spectra observed in above case, variations in the magnitude of radius have been accounted for by neither vertical nor horizontal shifts. This behaviour rules out the possibility of obtaining power spectra by moving a dominant spectrum vertically up or down when the radius of the vortex core changes. Fig. (3.10b) and (3.10d) show this behaviour quite evidently for Mexican-hat vortex and Gaussian vortex, respectively. Moving on to the position of inflexion in the spectra curves in Fig. $(\underline{3.10 \mathrm{~b}})$ and $(\underline{3.10 \mathrm{~d}})$, there exists an inflexion point on all the spectra but the spectrum of a larger radius, 0.4 , achieves this inflexion far earlier in the frequency range compared to the vortices with a smaller radius. Even though the velocity spectra of vortices of all sizes with the same directional strength 
tend to converge at more or less similar frequencies, larger vortices tend to decay energy levels at a much lower frequency. This can be interpreted as if the circulation of the same amount is concentrated in two different size vortices (one large and one small). The smaller vortex naturally has less volume to store that circulation unlike that of the larger radius vortex. Consequently, the core of the smaller vortex will become more concentrated than that of the larger vortex because the larger vortex has more volume to store the same amount of circulation. Further, the corresponding characteristics are observed in these plots through the location of inflexion points. While in a small vortex, the inflexion is found much later in the frequency range, and the inflexion point is found much earlier in the larger vortex due to the greater volume. So, by changing the radius of the vortex, spectra with different decay rate can be obtained which is of vital importance in realising a wider range of turbulent inflows which are not limited to turbulence based on the standard spectra by von Kármán etc.

\subsubsection{Inference}

In the results presented above, the ability to construct a turbulent inflow based on the correct statistics by manipulating the shape, strength, and size of the vortex is investigated. Turbulence is stochastic in nature; thus, a mathematical model used to describe it should possess some amount of randomness and stochasticity irrespective of the complexity of numerics involved. Earlier attempts $[19, \underline{21}, \underline{23}, \underline{144}, \underline{24}, \underline{39}, \underline{49}$ to create a turbulent inflow were either analytical in nature or lacked the randomness. By now the investigations have made it clear that a turbulent inflow based on a specific turbulence statistics can be constructed using vortices. However, it is not clear how many vortices will be required. The shape, strength and radius, spatial and temporal distribution are also not known. A rather brute force technique can be used where the vortices are arranged randomly in a finite space with values of strength and radius optimised $[54,56]$ to achieve the desired statistics. Such techniques present the problem of higher computational costs involved since at each time step the induced velocity due to each vortex needs to be calculated on the body, which is N-Body interaction at each iteration.

The significant findings from the above section concerning the statistical findings of a single vortex particle are:

i) a quantitative shift in the magnitude of the vortex strength is accounted in the spectrum through the shifting in the vertical direction of any principal spectrum,

ii) a change in the radius of the vortex is reflected through different decay rate in the spectrum,

iii) the ability to superimpose the disturbances associated with each particle, thus enabling the addition of all the disturbances.

These findings create an opportunity to devise a technique to formulate any turbulent velocity signal using a set of vortex particles with different strength and radius. A database can be created by storing the precomputed induced velocities of a group of vortices, with strengths and radii confined in an open or closed interval. The influences from the vortices with different radius are only required to be calculated, which then can be associated with the desired strength by just shifting the spectral density in the vertical direction. Further, the conclusive 


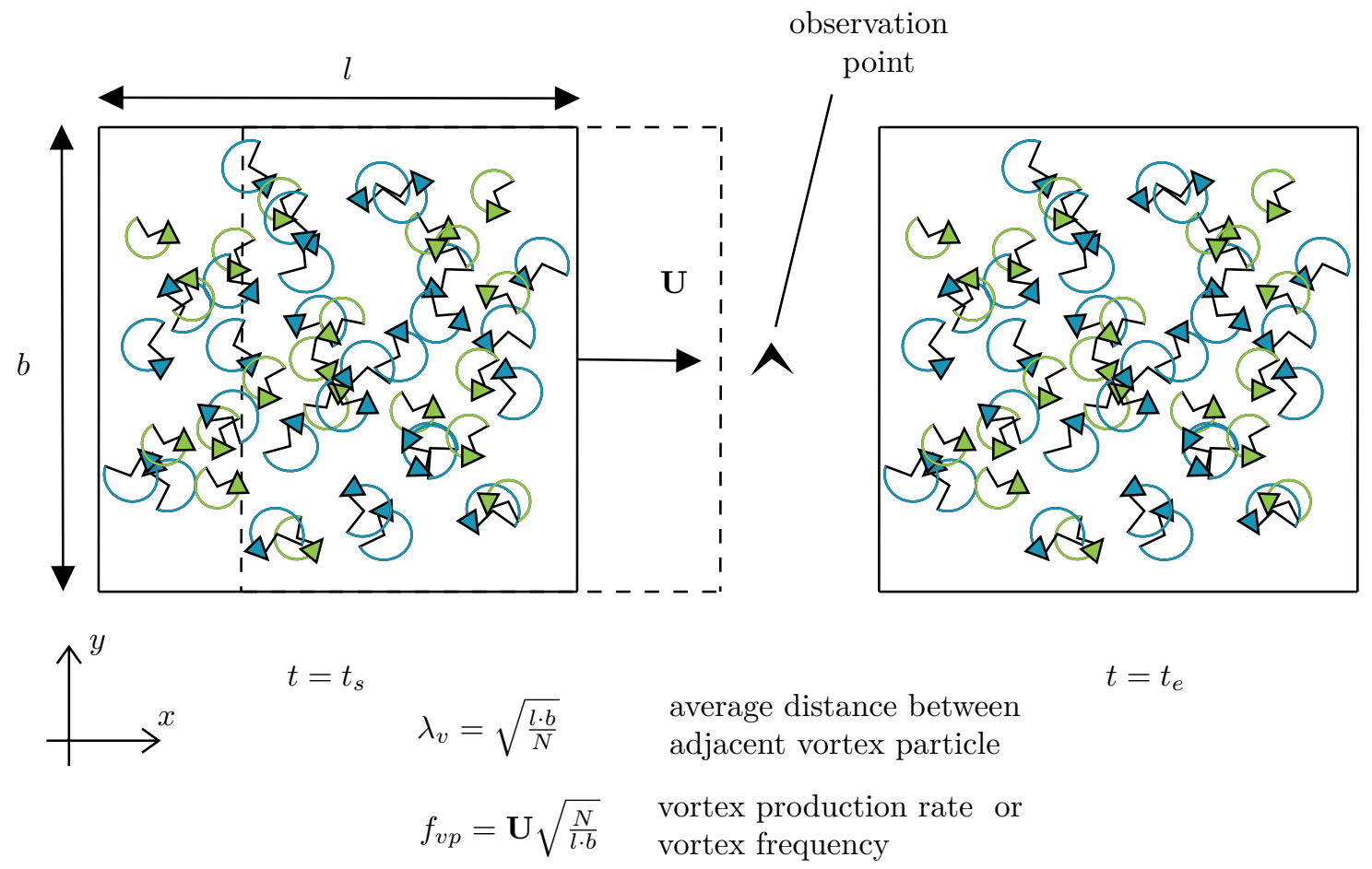

(a) convection of multiple vortices distributed randomly in rectangular space

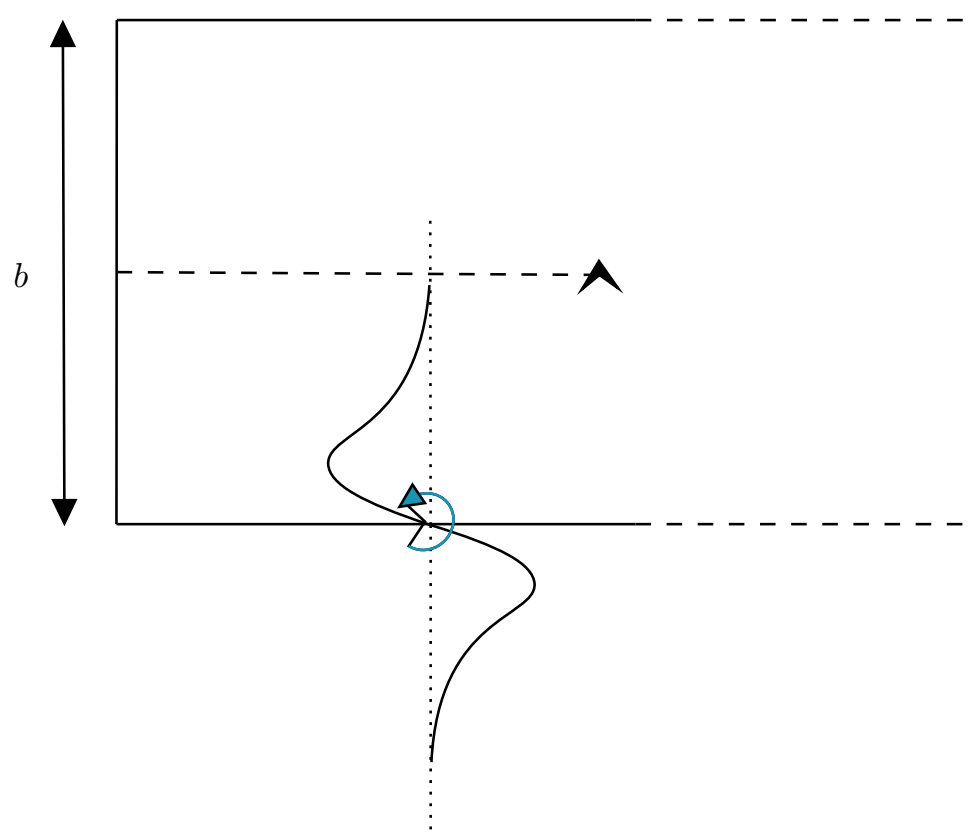

(b) width of the vortex window

Figure 3.11: Attributes of the vortex window 
Table 3.1: Vortex parameters controlling the turbulence statistics

\begin{tabular}{lll}
\hline Definition & $\begin{array}{l}\text { Stochastic } \\
\text { variable }\end{array}$ & Range \\
\hline Spatial distribution & $p(x), p(y)$ & {$\left[-x_{\min }, x_{\max }\right],\left[-y_{\min }, y_{\max }\right]$} \\
Strength distribution & $p(\Gamma)$ & {$\left[-\Gamma_{\min }, \Gamma_{\max }\right]$} \\
Radius distribution & $p(R)$ & $\left(0, R_{\max }\right]$ \\
\hline
\end{tabular}

signal is obtained by superimposing the influences of all the vortices involved to create the signal with proper time dilation.

\subsubsection{Multi-particle statistics}

Before constructing the database of precomputed velocity influences of vortex particles, it is of interest and importance to investigate the construction of a velocity signal with desired statistics by simply distributing the vortices in a two-dimensional finite space ${ }_{-}^{8} \mathcal{D} \subset \mathbb{R}^{2}$ with their parameters (strength,radius, and location).

Fig. (3.11a) shows a rectangular space, a vortex window, of a finite length $l$ and breadth $b$, where the vortices are distributed randomly. The window is controlled by three main parameters - (i) convection speed $U$, (ii) window area $A$, and (iii) number of vortices $N$. These three parameters together give the mean vortex production rate as $\sqrt{N / A} \cdot U$; and the last two collectively indicate an average distance between two adjacent vortex particles inside the virtual vortex window as $\sqrt{A / N}$. In this work, the length of the virtual vortex window is fixed at $l=100 c_{a}$ (refer to Appendix $\underline{B}$ ), and the width of the window is equal to the separation between the centre of the weakest and smallest vortex to the farthest point at which the corresponding fluctuation is induced as shown in the lower part of Fig. (3.11b). The determined length requires a vortex to travel for a duration of $100 / 5=20 s$, which is sufficiently long to conduct a statistical analysis. Particles do not share velocity influence among themselves, i.e. a particle is not influenced by the velocity field of the remaining parties and vice versa in the vortex window. At $t=0$, the vortex window is stationed at a point which is in farfield such that there is no induced velocity experienced at the observation point, $O$ at $(0,0)$. The window starts to move with a uniform velocity equivalent to the freestream velocity $U$ in the direction of $O$ at $t=t_{s}$. As the window approaches towards $O$, the observation point starts to experience induced velocities from all the vortex particles confined in the window. Since the event that is being attempted to be reconstructed, turbulence, with the help of Vortex particles is a random event, thus the variables governing the dynamics of these particles are also required to be random or stochastic in nature. To comprehensively understand the statistical behaviour of multiple vortex particles, it is important to first discuss the characterisation of these stochastic variables.

\footnotetext{
${ }^{8}$ Since the vortex particle method is based on an unbounded domain contrary to the conventional grid-based methods where vortices have been used to create such velocity fields under the widely studied topic of synthetic eddy modelling [141] which requires the definition of a precise volume (area for 2D) containing all the turbulent structures, a finite domain of interest is defined and named as vortex-window
} 


\subsubsection{Characterisation of stochastic variables}

A stochastic variable is a variable that is subject to variations due to random chance. This can be interpreted as the result of a random experiment where the stochastic variable, $x_{\text {stch }}$ as an example, has to be picked up from a given interval of number. Each time $x_{\text {stch }}$ is picked, a sample of the stochastic variable is obtained, and since the variable is stochastic, $x_{\text {stch }}$ is expected to have different values for multiple samples. In the present study, the stochastic variable is considered as a continuous stochastic variable that takes on any variable from a continuum interval. It is not possible to form a sum over such a set of numbers in a continuum interval since there are too many, because such a continuum is uncountable. Instead, the sum is replaced with an integral over the set of possible values. Hence, the stochastic variable assumes a continuous range of values, then a probability density function $p(x)[145,130]$ is defined as

$$
\begin{gathered}
p(x) \geq 0 \Rightarrow \int_{-\infty}^{\infty} p(x) d x=1 \\
P(x)=\int_{-\infty}^{\infty} p(\xi) d \xi \quad \frac{\mathrm{d} P(x)}{\mathrm{d} x}=p(x) .
\end{gathered}
$$

\subsubsection{Choosing an appropriate distribution}

Modelling a velocity signal using a set of discrete vortices require few constraints to regulate them to employ the stochasticity. A total of four stochastic variables, listed in Table $\underline{3.1}$, are introduced to control and optimise the overall distribution of random vortices to replicate two

Table 3.2: Special probability distribution functions [145]

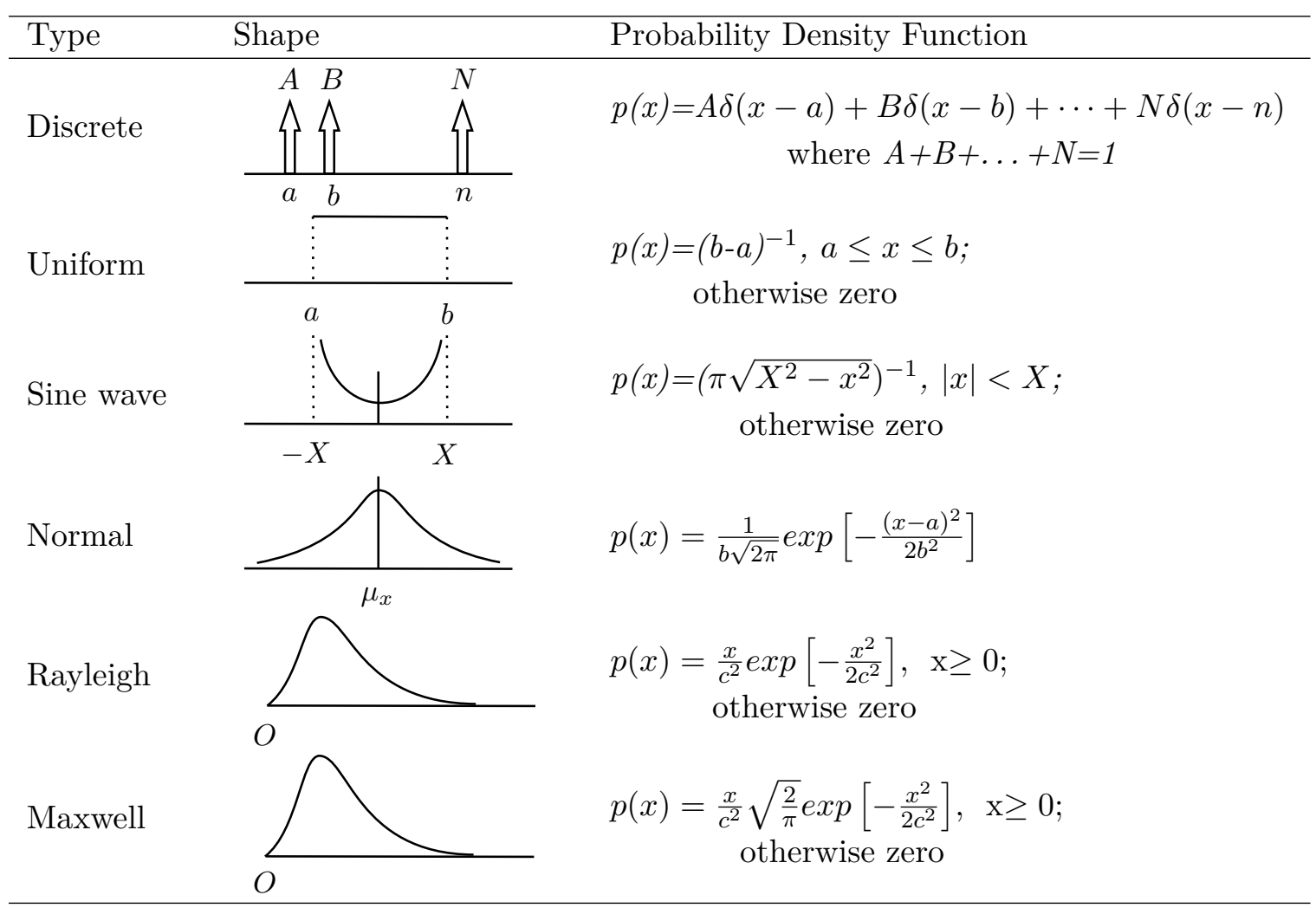


target energy spectra, Liepmann and von Kármán energy spectra, for a homogeneous isotropic two-dimensional turbulence. All the variables are defined by a probability density function, $\mathrm{PDF}$, (because of continuum range) $p(x)$. Determining the most appropriate distribution for a stochastic variable to achieve the desired statistics is an inverse problem where a custom distribution can be obtained by finding variable values using the target spectra. In the present work, instead of applying the approach of the inverse problem to figure out the probability distribution for the stochastic variable, an attempt is made to construct the velocity signal with desired statistics with the help of a few special PDFs given in Table. (3.2). The important things to know about the variables before assigning any PDF to them are:

i) Is the quantity discrete or continuous?

ii) Does the quantity have bounds?

iii) How many modes does it have?

iv) Is it symmetric?

In the next section, all the four variables given in Table. (3.1) are tested for all the four questions before assigning a few PDFs from Table. (3.2) to each one of them.

Spatial distribution Variable $x$ and $y$ identify the abscissa and ordinate of the coordinates of the vortex position, respectively, and their possible outcomes are based on the number of vortices $N$ present in the vortex window. Next is to identify the characteristics of $x$ and $y$ based on the queries mentioned in the last section. Since $x$ and $y$ are represented by real numbers and have $N$ possible values between any two values in a specific domain, it is convenient to use a continuous PDF because the number of vortices must be an integer, it would be convenient to represent uncertainty about the number with a continuous probability distribution. Essentially the vortices are frozen in a confined space, and it can be comfortably asserted that there is zero probability that the quantity would be smaller than some lower bound, or larger than some upper bound. For abscissa, the lower bound is chosen $x_{\min }=-l / 2$, and the upper bound $x_{\max }=l / 2$. Similarly, for ordinate lower and upper bounds are chosen $y_{\min }=-b / 2$ and $y_{\max }=b / 2$. Many standard continuous probability distributions, such as the normal distribution, are unbounded. Nevertheless, the probability density drops off quite rapidly for extreme values, with near exponential decay, in fact, for the normal distribution ${ }_{-}^{9}$. If the distance between the two vortices is zero, the vortices overlap entirely in such a way that the circulation calculated on a surface containing both becomes null for vortices with equal and opposite directional strength, becomes double for vortices with equal and same directional strength [147]. Since there is homogeneous turbulence, the variable's probability should not require more than one mode. The PDF is kept symmetric for simplicity. Hence, $x$ and $y$ are defined with uniform and normal distributions, as shown in Table. (3.3). The two variables will be used to create a realistic turbulent velocity field in later sections. The PDFs for $x$ and $y$ can be written as

\footnotetext{
${ }^{9}$ Accordingly, unbounded distributions are widely used to represent real-world quantities that have finite bounds. For example, the normal distribution generally provides a good fit for the distribution of heights in a human population, even though it might be certain that no person's height is less than zero or greater than 12 feet $[146]$.
} 
Table 3.3: Probability Density Functions for stochastic variables based on their characteristics discussed in section

\begin{tabular}{llllll}
\hline variable & i) & ii) & iii) & iv) & PDF \\
\hline$p(x)$ & $\mathbf{Q}$ & $\mathbf{\square}$ & $\mathbf{\square}$ & Uniform, Normal \\
$p(y)$ & $\mathbf{Q}$ & $\mathbf{\square}$ & $\mathbf{\square}$ & Uniform, Normal \\
$p(\Gamma)$ & $\mathbf{\square}$ & & $\mathbf{\square}$ & Normal, Double-exponential \\
$p(R)$ & $\mathbf{\square}$ & & & Rayleigh, Maxwell, Exponential \\
\hline
\end{tabular}

Uniform distribution

$$
\begin{aligned}
& p_{\mathcal{U}}(x)=\left\{\begin{array}{cc}
\left(x_{\max }-x_{\text {min }}\right)^{-1} & \forall x_{\text {min }} \leq x \leq x_{\text {max }} \\
0 & \text { otherwise }
\end{array},\right. \\
& p_{\mathcal{U}}(y)=\left\{\begin{array}{cc}
\left(y_{\max }-y_{\min }\right)^{-1} & \forall y_{\text {min }} \leq y \leq y_{\max } \\
0 & \text { otherwise }
\end{array}\right.
\end{aligned}
$$

Normal distribution

$$
\begin{aligned}
& p_{\mathcal{N}}(x)=\frac{1}{\sqrt{2 \pi \sigma^{2}}} e^{-\frac{(x-\mu)^{2}}{2 \sigma^{2}}}, \quad \text { where } \quad \sigma=\left(\frac{x_{\max }+x_{\min }}{2}\right) \quad \text { and } \quad \mu=0, \\
& p_{\mathcal{N}}(y)=\frac{1}{\sqrt{2 \pi \sigma^{2}}} e^{-\frac{(y-\mu)^{2}}{2 \sigma^{2}}}, \quad \text { where } \quad \sigma=\left(\frac{y_{\max }+y_{\min }}{2}\right) \quad \text { and } \quad \mu=0 .
\end{aligned}
$$

$\Gamma$ distribution The circulation strength of the vortices are stored in $\Gamma$ variable, and the possible outcomes are based on the number of vortices. In order to consider a large number of vortices in the domain $\mathcal{D} \subset \mathbb{R}^{2}$, a continuous distribution should be used. The value of strength variable $\Gamma$ is based on the results of experimental investigation $[148,149,150,147$, 151], and it is observed that its value depends heavily on the vorticity present in the domain. Hence, for simplicity, the value of $\Gamma$ in this study is confined between the lower bound equals to -1.5 and the upper bound equals to 1.5. However, in order to consolidate the randomness observed in the circulation magnitude in the experiments mentioned earlier, it is decided to consider an unbounded distribution, besides a bounded one, with a standard deviation $\sigma=\Gamma_{r m s}=1.0$. Rast and Pinton [152] in his work on point vortices injected the vortices at a constant rate, with individual vortex intensities Gaussianly distributed with zero mean and standard deviation $\Gamma_{r m s}$. It is crucial to know here that the chosen standard deviation is not exhaustive; instead, the distribution can be reconstructed by playing around with the standard deviation here. Hence $\Gamma$ is defined with normal and double-exponential distribution, as shown in Table 3.3. The PDFs have their peak at the standardised mean, and its "spread" increases with the standard deviation, which is equal to unity for the normal distribution and with an exponential decay equals to unity for double-exponential distribution. The PDFs for $\Gamma$ can be written as 
Uniform distribution

$$
p_{\mathcal{N}}(\Gamma)=\frac{1}{\sqrt{2 \pi \sigma^{2}}} e^{-\frac{(\Gamma-\mu)^{2}}{2 \sigma^{2}}}, \quad \text { where } \quad \sigma=1 \quad \text { and } \quad \mu=0
$$

Double-exponential distribution

$$
p_{\mathcal{L}}(\Gamma)=\frac{1}{2 \lambda} e^{\left(-\frac{|x-\mu|}{\lambda}\right)}
$$

Note: The vortex configurations are not checked with $\Gamma$ distributed using double-exponential distribution at present. It can be considered later in future while extending the method for three-dimensional study.

Radius distribution The radius of the vortex is represented by $R$, which is defined for a shape function a vortex in Eq. (3.52-3.53). The possible outcomes of radius values are definitely non-negative-valued random variables bounded on a semi-infinite range with the lower bound fixed at zero (open interval). The possible distribution for $p(R)$ is then Rayleigh 10 with scaling parameter $\sigma \in\{0.1,0.2,0.3\}$. (Maxwell, and Exponential distribution can also be considered in future to study their influence on vortices.)

$$
p_{\mathcal{R}_{\mathcal{Y}}}(R)=\frac{R}{\sigma^{2}} e^{\frac{-R^{2}}{2 \cdot \sigma^{2}}}, \quad \forall R \geq 0, \quad \sigma=\{0.1,0.2,0.3\},
$$

In this section, the probability distributions for the spatial distribution, the strength and the radius of the vortices are chosen. $p(x)$ and $p(y)$ have two options to choose from, whereas $p(\Gamma)$ and $p(R)$ are distributed with one PDF each. This results in 4 possible combinations based on which the vortices can be scattered in the vortex window to study their behaviour. This is to identify the best suitable combination of PDFs for all the stochastic variables to realise a turbulent velocity signal fulfilling the desired statistics.

\subsubsection{Creating realistic $2 \mathrm{D}$ turbulence}

The resulting combinations form the choices of PDFs stated in the earlier section are written using a series of alphabets, four to be exact $-(\alpha \beta \gamma \theta)$, which depicts the initials of the PDFs. The first alphabet is describing the distribution for abscissa $p(x)$, second alphabet for ordinate $p(y)$, third alphabet for the strength $(\Gamma)$, and the fourth alphabet for the radius, $p(R)$, of the vortex particle. As an example, a possible combination is UUNR, which means the particles are spatially distributed uniformly with their strength distributed normally, and the radius is distributed in Rayleigh distribution. For the present analysis, four combinations based on the PDFs mentioned in Table. $\underline{3.3}$ ) are chosen which are then used with the Gaussian and the Mexican-hat vortices, separately, with total number vortices $N \in\{200,400,800\}$. This is to be noted that the values of $N$ are chosen randomly, and can be modified for better results later on. The target spectra for the analyses is Liepmann spectra which are used as the target curve to fit the energy spectra due to the velocity fluctuations generated by the vortices. The algorithm to fit the curves uses the Levenberg-Marquardt algorithm [153, 154, 155] (Appendix C).

\footnotetext{
${ }^{10}$ The Rayleigh distribution is a special case of the Weibull distribution with a scale parameter of 2 . When a Rayleigh is set with a shape parameter $(\sigma)$ of 1 , it is equal to a chi-square distribution with 2 degrees of freedom
} 
Curve fitting finds the values of the coefficients (parameters) which make a function match the data as closely as possible. The best values of the coefficients are the ones that minimise the value of Chi-square. Chi-square is defined as:

$$
\chi^{2}=\sum_{i}^{n}\left(\frac{y-y_{i}}{\sigma_{i}}\right)^{2}
$$

where $y$ is a fitted value for a given point, $y i$ is the measured data value for the point and $\sigma_{i}$ is an estimate of the standard deviation for $y_{i}$. Eq. ( $\underline{3.69)}$ represents the expression for $\chi^{2}$ to be minimised to fit the spectral density of the longitudinal component of the generated velocity field with the Liepmann target curve, $E_{11}$.

$$
\chi^{2}=\sum_{i}^{n}\left(\frac{f_{i}^{u}-\left(\frac{u_{r m s}^{\prime 2} \lambda}{\pi} \frac{1}{\left(1+4 \pi^{2} \lambda^{2} f_{1}^{2}\right)}\right)_{i}}{\sigma_{i}}\right)^{2} .
$$

$f_{i}^{u}$ is the frequency of the generated velocity fluctuations, and $\sigma_{i}$ are the uncertainties in the $f^{u}$. These are used as weights in the least-squares problem, i.e. minimising $\chi^{2}$. For simplicity, the uncertainties are assumed to be 1 .

\subsubsection{Gaussian vortices}

First, the behaviour of Gaussian vortices based on the combinations is studied. The clustered vortices are arranged in the vortex window which is convecting with $U=5 \mathrm{~m} / \mathrm{s}$, aligned with $x$-axis. The streamwise length of the vortex window is equal $100 c_{a}$, whereas the cross-flow length is equal to $0.2 c_{a}$. The PDF controlling parameters are tabulated in Table. (3.4).

UUNR UUNR means $p(x)$ is distributed uniformly, $p(y)$ also uniformly, $p(\Gamma)$ normally, and $p(R)$ in Rayleigh fashion. Fig. (3.12) shows a set of vortices, represented by circular disks in green colour indicating negative (clockwise flow) circulation, and blue symbols indicating positive (counterclockwise flow) circulation, respectively, distributed in a uniform fashion in the vortex window. The window starts to convect at $t=0$ with time-step equals $\Delta t=0.001$, which is also the time between successive samples and is referred to as the sampling period. This sampling period allows capturing the spectra in the bandwidth range of $0-500 \mathrm{~Hz}_{-}^{11}$. The

\footnotetext{
${ }^{11}$ Interested readers are referred to a standard text on signal processing by Oppenheim and Verghese [156]
}

Table 3.4: Vortex parameters controlling the turbulence statistics

\begin{tabular}{llll}
\hline Definition & $\begin{array}{l}\text { Stochastic } \\
\text { variable }\end{array}$ & Distribution & Range \\
\hline Spatial distribution & $p(x), p(y)$ & Uniform & $\begin{array}{c}x_{\min }=-l / 2 \\
x_{\max }=l / 2 \\
\end{array}$ \\
& & & $\begin{array}{c}y_{\min }=-b / 2 \\
y_{\max }=b / 2\end{array}$ \\
Strength distribution & $p(\Gamma)$ & Normal & $\sigma=1.0$ \\
Radius distribution & $p(R)$ & Rayleigh & $\sigma=0.1$ \\
\hline
\end{tabular}




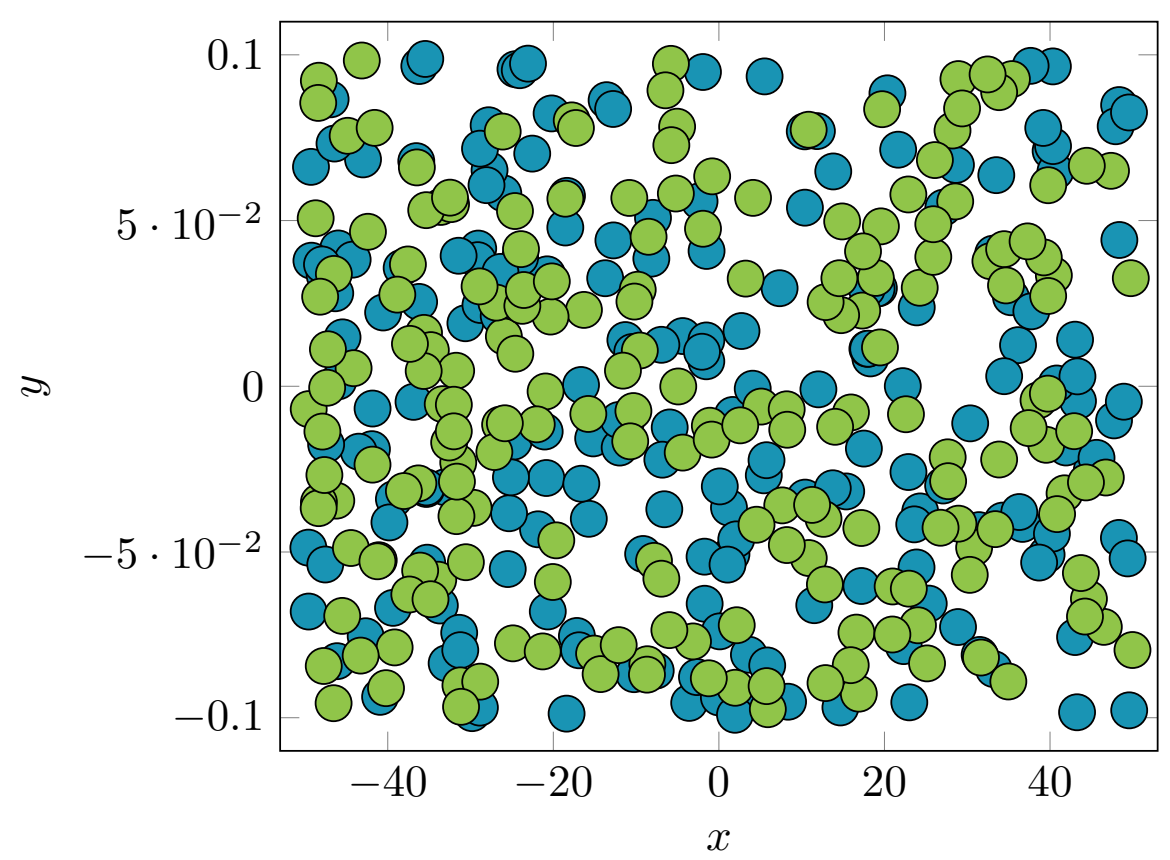

Figure 3.12: Vortices arranged in UUNR configuration; green symbols indicate negative (clockwise flow), and blue symbols indicate positive (counterclockwise flow) circulation, respectively.

left-hand side plots are velocity fluctuations ${ }^{12}$ for each velocity component, and the right-hand side plots are the energy spectra for each velocity component. The vortex window is convected three times, with three different number of vortices each time. Fig. (3.18a)-(3.18b) shows the velocity fluctuations and energy spectra for 200 vortices, respectively. In the lower frequency range, the transverse velocity spectrum is far from the desired statistics, Liepmann spectrum for vertical velocity for two-dimensional turbulence. However, the horizontal component behaves quite well and matches with the Liepmann horizontal velocity spectrum. At higher frequencies, the vortex generated velocity signal decays much faster than that of Liepmann velocity. This can be understood from the fact that the number of vortices in the window is less as per the requirement, and at the same time with the ability to realise the characteristic shape of the spectra while considerable variation of slopes can be seen in the curves, it can be expected that increasing the number of vortices can lead to better agreements. With $N=400$, a better agreement is observed between the horizontal as well as the vertical velocity components with the Liepmann spectra, shown in Fig. (3.18d). By further increasing the number of vortices to 800 , a fairly stable agreement for both the horizontal and the vertical components of the velocity is achieved at a lower frequency. However, no change is observed between the spectra mismatch at higher frequencies for $N=400$ and $N=800$ as can be seen evidently in Fig. (3.18f). However, at this moment, it is too early to say that $U U N R$ combination is not able to capture spectra at higher frequencies because the number and strength of the vortices can still be modified to obtain the desired statistics.

UNNR In $U N N R$ configuration, the spatial distribution is shown in Fig. (3.13). The abscissa $x$ is distributed uniformly, whereas, the ordinate $y$ is distributed normally. The strength and radius distributions are unchanged and similar to the earlier configuration,

\footnotetext{
${ }^{12}$ The fluctuations are recorded from the instance when the vortex window hits the observation point until it fully convects through the same point. This is shown in Fig. (3.11).
} 


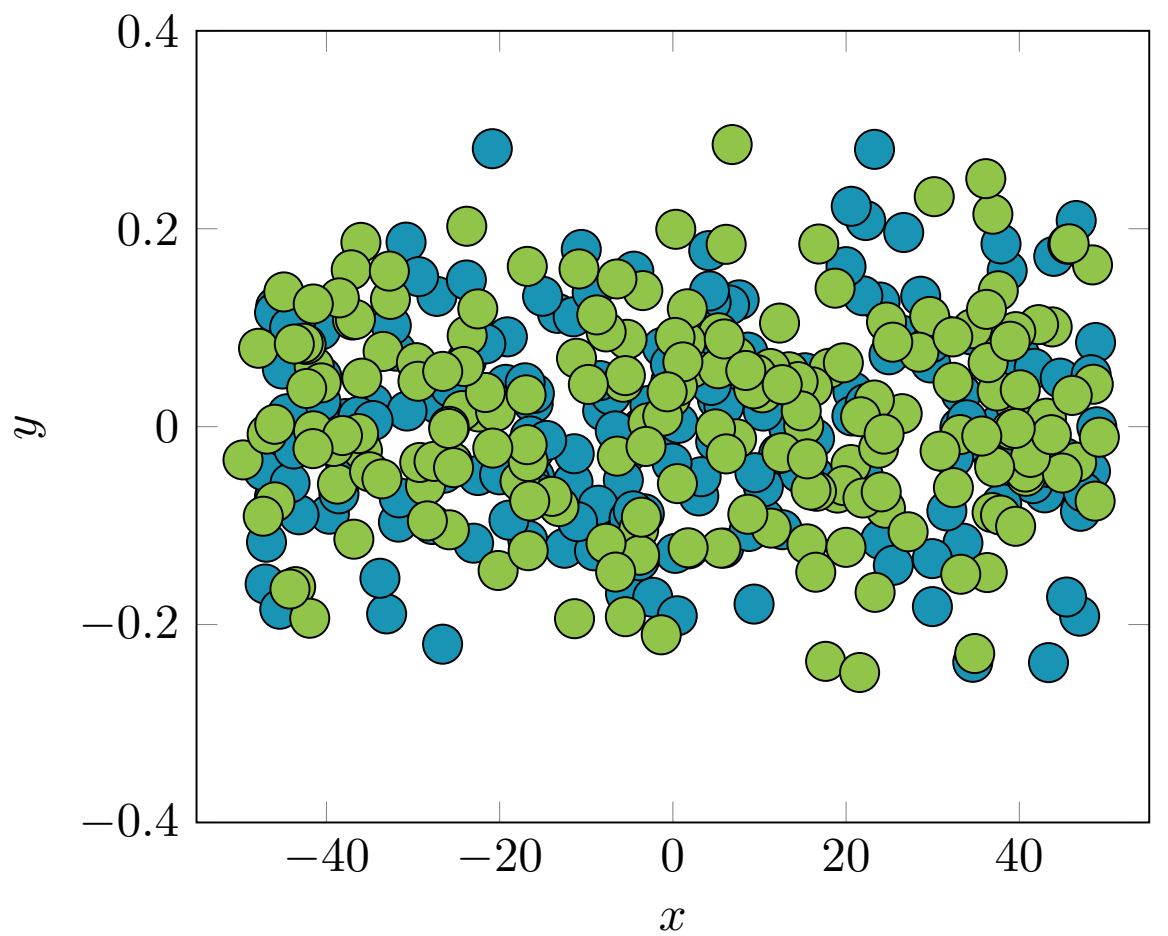

Figure 3.13: Vortices arranged in UNNR configuration; green symbols indicate negative (clockwise flow), and blue symbols indicate positive (counterclockwise flow) circulation, respectively.

normal and Rayleigh, respectively. Similar to the earlier case, the window is convected three times with three different number of vortices each time. Fig. (3.19a)-(3.19b) shows the velocity fluctuations and energy spectra for 200 vortices, respectively. Alike the corresponding case for $U U N R$, the vertical velocity spectrum is showing lower levels of energy compared to the target spectrum in the lower frequency range. However, the inflexion points for all the spectra seem to coincide well, but at higher frequencies, the artificial velocity spectra decay quite faster. This behaviour is mitigated, not completely, by increasing the number of vortices to 400, as shown in Fig. (3.19d). Further increasing the vortices to 800 captures a good agreement of spectra at lower frequencies, but the faster energy decay at higher frequencies can still be observed.

NUNR The spatial distribution for $N U N R$ is shown in Fig. (3.14). The behaviour of all the spectra at all the values of $N$ is similar to the spectra obtained in previous configurations. However, the gap between the vertical velocity component spectra does not seem to reduce in all the cases in $N U N R$ configuration shown in Fig. (3.20). The horizontal velocity spectra show a good agreement with the Liepmann spectra at all the values of $N$. Nevertheless, the window can be convected with more number of vortices in later cases to see if this discrepancy in the horizontal spectra still exists.

NNNR $N N N R$ designates PDF as normal for spatial distribution, normal for strength, and Rayleigh for the radius of the vortices. Normal distribution for abscissa and ordinate seem to be the densest relatively, as shown in Fig. (3.15). Moving on to the spectra, Fig. (3.21b), the vertical velocity component spectrum shows a greater deviation from the target spectrum for $\mathrm{N}=200$ at lower frequencies. However, an increase in the number of vortices from 200 to 400 


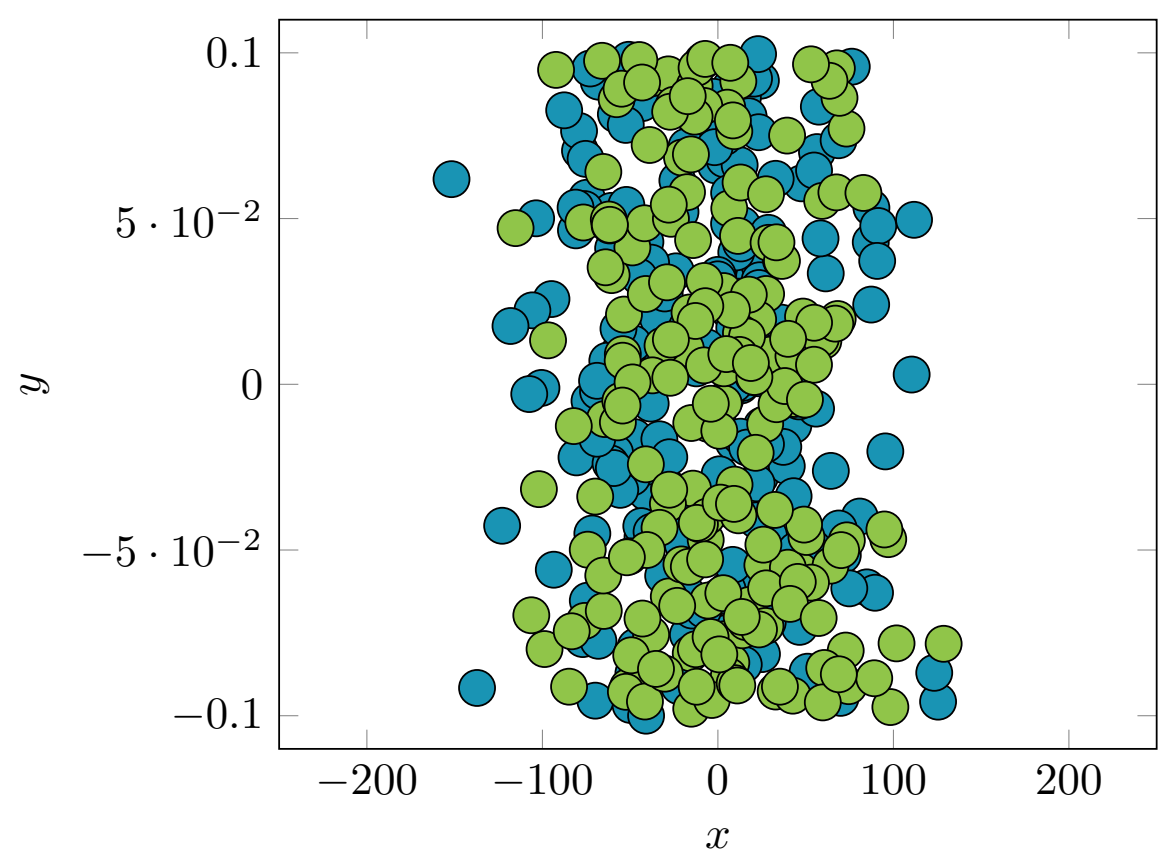

Figure 3.14: Vortices arranged in NUNR configuration; green symbols indicate negative (clockwise flow), and blue symbols indicate positive (counterclockwise flow) circulation, respectively.

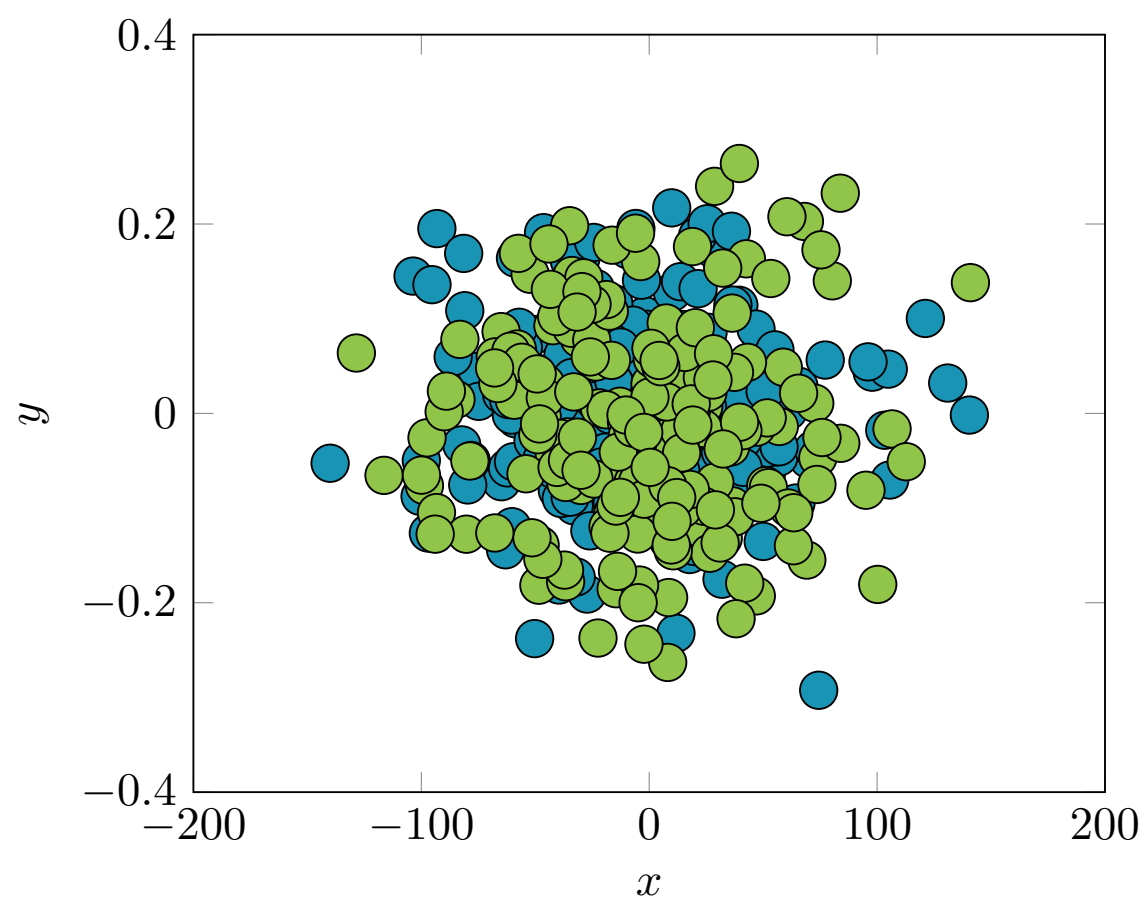

Figure 3.15: Vortices arranged in NNNR configuration; green symbols indicate negative (clockwise flow), and blue symbols indicate positive (counterclockwise flow) circulation, respectively.

and 800 reduces this deviation as can be seen in Fig. (3.21d-3.21f). Following the trend for an early decay in energy at higher frequencies, horizontal as well as the vertical velocity spectra of the vortex window with $\mathrm{N}=200$ decay much earlier, the spectra begin to drop as soon as the inflexion is reached.

The investigation above infers that energy spectra can be matched to a more extended range in frequency bandwidth by uniformly distributing the vortices. Using at least 200 vortices, it is observed that the spectral densities of the artificial velocity field resemble the characteristic 
shape of the Liepmann spectra. The spectra with 400 vortices of each case seem to prove to be much better in energy distribution than the rest of the cases, whereas at larger frequencies, the number of vortices and their strength can be observed to decrease the energy level rapidly. This study also exhibits the fact that the Gaussian vortex is well suited to replicate the energy spectra for lower frequency range.

\subsubsection{Mexican-hat vortex}

This section presents the behaviour of Mexican-hat vortices based on the possible PDFs for the spatial, strength, and radius of the vortices. The vortex window, filled with the vortex particles mollified by Mexican-hat shape kernel, is convected in the streamwise direction with the freestream velocity $U=5 \mathrm{~m} / \mathrm{s}$, aligned with the $x$-axis. The time step for the investigation is $\Delta t=0.001$ to produce spectra in the bandwidth of 0-500 Hz. The streamwise length of the vortex window is equal $100 c_{a}$, whereas the cross-flow length is equal to $0.2 c_{a}$. The PDF controlling parameters are tabulated in Table. (3.4).

UUNR Three windows with different particle densities are studied here, similar to the previous case of the Gaussian vortex particles. UNNR contains the vortices with their abscissa and ordinate distributed uniformly across the window. The least dense window with $\mathrm{N}=200$ particles, Fig. (3.22b), shows a deviation in the vertical velocity component spectrum at lower frequencies, which mitigates with increasing frequencies but at the cost of fluctuations mid-way the spectra. This deviation is expected to be a result of less number of particles in the window whereas, the fluctuations, which were not witnessed in the Gaussian vortices, can be explained by the presence of two inflexions in the velocity profile of the Mexican-hat waveform vortex particle as shown in sec. (3.6.3). By increasing the number of vortices, the fluctuations are observed to mollify as shown in Fig. (3.22d) which further smoothens out with $\mathrm{N}=800$ as shown in Fig. $\underline{3.22 \mathrm{f})}$. It can be inferred here that the fluctuations in the spectra are occurring in the cases with limited number of vortices in the domain which results in a larger space $\lambda_{v}$ between the particles. At higher frequencies, the artificial velocity spectra, for all the cases of $N$, decays much earlier than the target spectra. However, the possibility of replicating a characteristic spectral shape with fewer deviations with UUNR distribution is observed, which can further be examined to achieve more complex turbulent inflow signals.

UNNR The UNNR configuration has not proved to be suitable for replicating target energy spectra using Mexican-hat vortices. Spectra for both the horizontal and vertical velocity component do not appear to match with the Liepmann spectra for all the cases, as shown in Fig. (3.23). Curve fitting optimisation is carried out with 200 vortices, but a considerable difference in energy levels is observed as shown in Fig. (3.23b). Moreover, for $N=400-$ Fig. (3.23d), it is found out that spectra of artificial velocities around 100 Hertz start to fluctuate exceptionally. The identical behaviour is detected in the previous configuration too. Fig. (3.23f), which presents the spectral densities of velocities due to 800 vortices whose abscissa are distributed uniformly, and ordinates are normally, shows that both components of the artificial velocity have more energy than that of the target one. It is also to be noted that in this case, many iterations were needed for the optimisation. 
NUNR The results of the NUNR configuration are similar to the results of the $U N N R$ configuration. The spectral density of longitudinal velocity with 200 vortices in this combination is showing considerable harmony in the lower frequency range with the target energy spectra, while the decay in energy is also seen to be delayed in the higher frequency range as shown in Fig. (3.24b). However, the transverse spectra do not show any agreement with the target in the lower frequency range. The spectral density of longitudinal velocity with 400 vortices is seen to fluctuate heavily as can be seen in Fig. (3.24d), which may be due to overlapping of vortices of the same directional strength. Moreover, at the same time, in the lower frequency range, the longitudinal component seems to maintain harmony with the expected target spectral shape. The behaviour observed with 800 vortices is shown in Fig. (3.24f). There is a constant distance between the spectral densities of $u$ and $u_{\text {target }}$, which shows the difference in the energy levels. This behaviour seems to be decreasing as one approaches the intersection point of longitudinal and transverse target spectrum near $100 \mathrm{~Hz}$, but after that, the vortices begin to decay their energy following the typical trend observed in all the cases so far. Similarly, the artificial transverse spectra exhibit an offset characteristic to the target spectra on a wider frequency range.

NNNR The NNNR configuration is showing a good agreement in the spectral density of longitudinal and transverse velocity with 200 vortices in the lower frequency range with the target energy spectra, while the energy decay is also seen to be delayed in the higher frequency range as shown in Fig. (3.25b). The spectral density of horizontal velocity with 400 vortices is seen to fluctuate heavily as can be seen in Fig. (3.25d), which may be explained by a similar reason stated above. Moreover, at the same time, in the lower frequency range, the horizontal component seems to be in good agreement with the expected target spectral shape. The behaviour of $u$ observed in lower frequency with 400 vortices can be seen as repeating itself for 800 vortices in Fig. (3.25f). A constant offset between the spectral densities of $v$ and $v_{\text {target }}$, which shows the difference in the energy levels is observed until the inflexion point of all the spectral densities are reached.

Overall, this investigation shows that UUNR is the most appropriate combination to replicate target spectra using Mexican-hat vortices. Energy degradation is observed in higher frequencies, but by increasing the number of vortices and their strength, the target spectra can be obtained on a broader frequency range.

\subsubsection{3 $\mathrm{N}=10000$}

Scattering 200 vortices into the vortex window results in an injection of 15.81 vortices at each time step in the vortex window. At this rate, artificial velocity constructed using both the Gaussian and Mexican-hat vortices do not match the Liepmann target spectra, although both the longitudinal and transverse spectra of the velocity field have been able to mimic the characteristic shape of the target spectrum for two-dimensional turbulence. The UUNR combination for the vortices, in which abscissa is uniformly, and the ordinate is uniformly distributed, with the vortex strength normally, and the radius is distributed according to Rayleigh distribution has proven to be the most appropriate and successful distribution in reconstructing a velocity signal with desired statistics. In Fig. (3.16a) and (3.16b) , the 


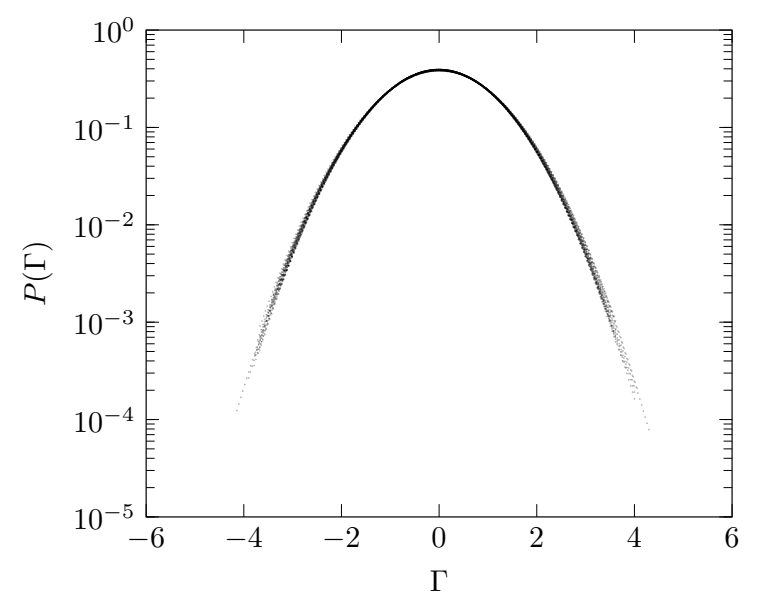

(a) vortex strength $\mathrm{PDF}$

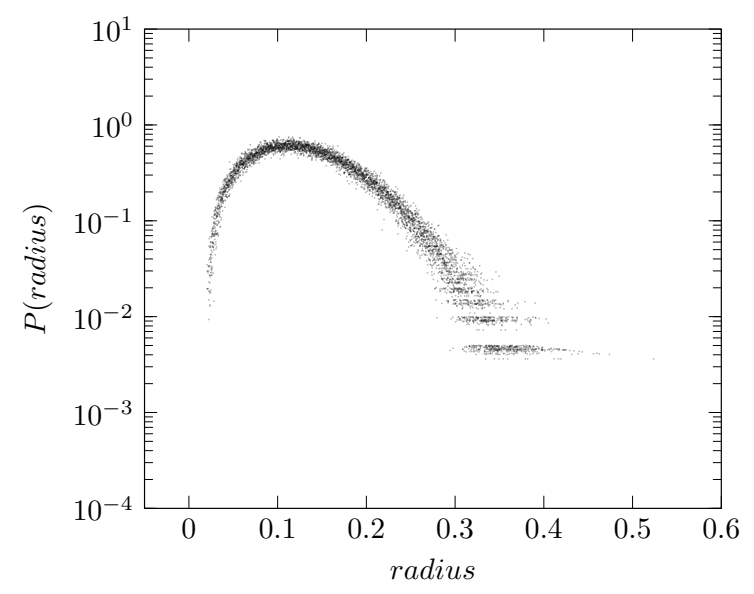

(b) vortex radius PDF

Figure 3.16: PDFs obtained from 300 iterations of random circulation distribution. Values are normalised by their standard deviation, and the total probability density integrates to unity

vortex strength and vortex radius PDFs obtained from the time histories of 300 iterations are visualised. Values are normalised by their standard deviation, and the total probability density integrates to unity. A further investigation is carried out with a higher vortices production rate in this section, and it is found out that keeping this rate above 110 is highly stable in producing the same spectra repeatedly. The table below (Table. $(\underline{3.5})$ ) mentions the number of vortices and the corresponding vortex production rate derived from them. Spectral analysis of their longitudinal and transverse velocities is shown in Fig. (3.17) by scattering the 10000 Gaussian and Mexican-hat vortices according to UUNR distribution at 110 vortices injected per time-step interval. The velocities are produced 300 times which is sufficient to conduct the statistical analysis using the power spectrum. Vortex particles mollified by Mexican-hat wavelet conveniently reproduce the velocity spectra with desired statistics using the curve fitting optimisation. It was observed that at $\mathrm{VPR}=110$, the same results can be obtained repeatedly for Liepmann spectra, Fig. (3.17b), and von Kármán spectra, Fig. ( $\underline{3.17 \mathrm{~d}})$, for twodimensional turbulence, respectively. While Gaussian vortices can replicate the same velocity profile over 300 iterations, the power spectral density of their induced velocity fluctuations is unable to match the expected statistical properties. As can be seen in Fig. (3.17a) - (3.17c), despite having 10000 vortices, the same strength and size as of Mexican-hat shaped vortices, the Gaussian vortices have been able to create a weaker energy distribution over the frequency range relative to the target energy spectra.

Table 3.5: Vortex parameters controlling the turbulence statistics

\begin{tabular}{ll}
\hline No. of vortices & $f_{v p}$ \\
\hline 200 & 15.81 \\
400 & 22.36 \\
800 & 31.62 \\
1000 & 35.35 \\
5000 & 79.05 \\
10000 & 111.80 \\
\hline
\end{tabular}


This investigation suggests that Mexican-shaped vortices are superior to Gaussian vortices in developing the expected statistics over a wider frequency range. But the important thing to note here is that this whole investigation depends on stochastic variables, i.e. the values using which Mexican-Hat is producing successful results are not necessarily those values which can make the Gaussian vortex, or any other vortex mollified using a wavelet function to produce the same results. Interested readers are encouraged to vary vortex strength and radius with different vortex production rate as well as distinct types of vortex models in this model to generate velocity profiles for vortex particle method.

The research conducted so far is necessary to investigate the relationship between the vortex parameters and the variables that characterise the turbulence, which is necessary for this research. The foundation of this entire chapter rests upon the ability of a group of vortices to represent a random velocity field. It is crucial to enumerate the important findings so far before moving on to the next section.

i) Investigating the building block of vortex methods, a point vortex in two-dimensional space, and the relationship between its streamfunction and the velocity field induced by it. It presents the singularity associated with a point vortex, which is also shown by the case where multiple vortices, four to be precise.

ii) To deal with singularity associated with the point-vortex, the concept of cut-off function is used in which a mollified kernel is constructed with the help of Gaussian and Mexican-hat cut-off function. This leads to the omission of non-physical values of induced velocity being experienced by a point approaching the centre of the vortices.

iii) A parametric study of these mollified vortex-induced velocity fields is then performed, where the difference in velocity fields is shown by varying the vortex strength and radius.

iv) The next section studies the statistics of the parametric study of the cases presented in the earlier case by referring to the simple correlation functions used to quantify the statistics of a single particle. This section discussed and demonstrated the possibility of reconstructing a random velocity field generated by convecting restricted or frozen vortices within an imaginary rectangular window of finite dimensions. To introduce the stochasticity (since placing the vortices at fixed coordinates in the vortex window all the time seems rigid and also restricts the possibility of generating highly complex velocity signals) in the generated velocity field, the vortex control parameters - spatial coordinates, strength, and radius use stochastic variables which distribute the samples within the space using standard probability distributions.

Now the question arises that how are these stochastic variables, which control the vortices parameters, related with the real atmospheric turbulence parameters - turbulence intensity and length scale.

\subsubsection{Association between vortices and turbulence}

The ability of artificial turbulence, generated by vortices, to realise the desired statistical characteristics depends on two parameters: maximum vortex radius, $R_{e}$, and standard deviation 


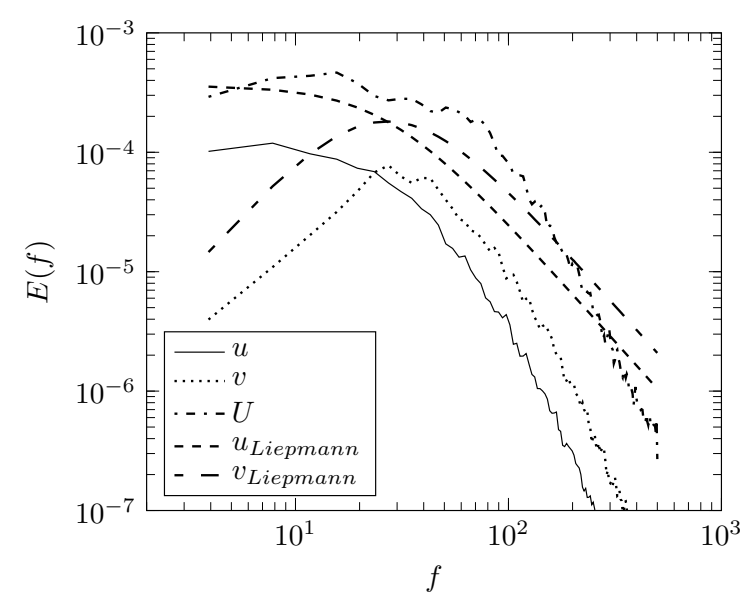

(a) Gaussian-Longitudinal-Liepmann

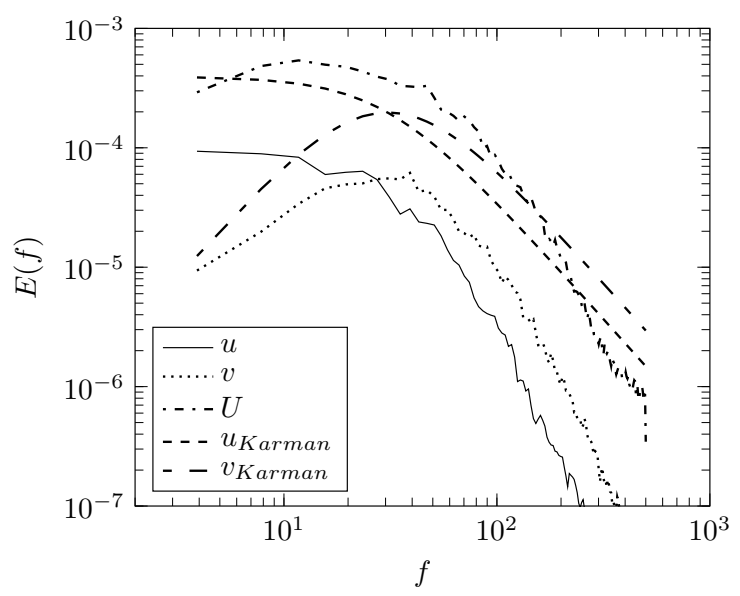

(c) Gaussian-Transverse-Kármán

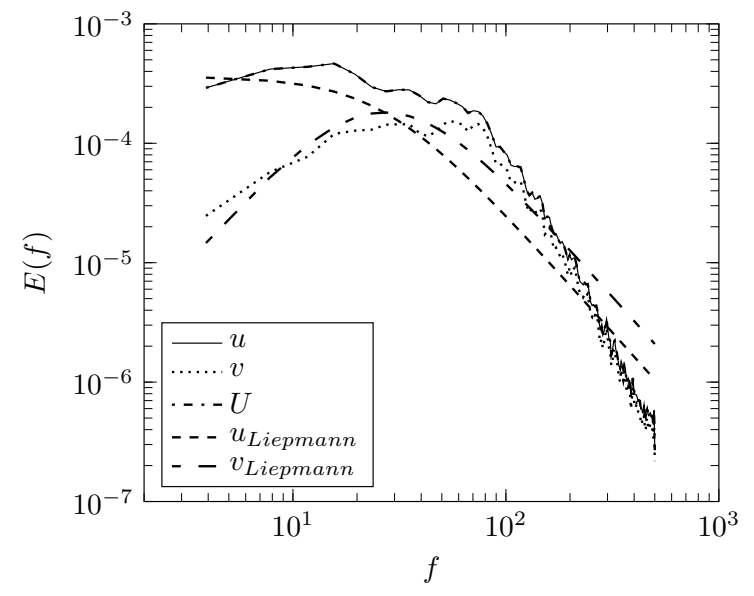

(b) Mexican-hat-Longitudinal-Liepmann

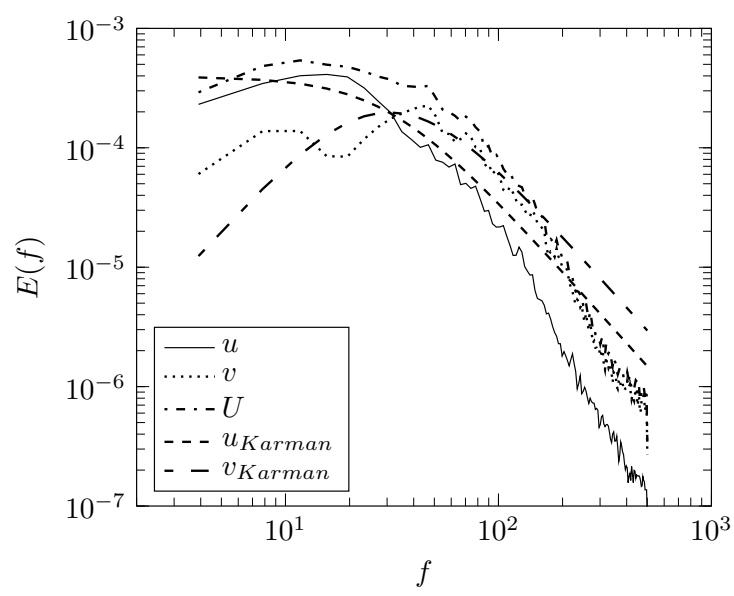

(d) Mexican-hat-Transverse-Kármán

Figure 3.17: Velocity spectra UUNR Gaussian Mexican-hat 


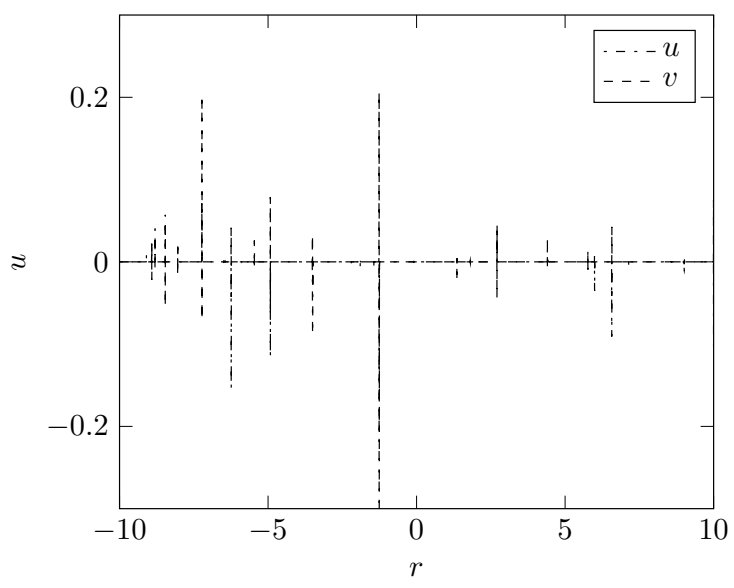

(a) velocity-fluctuations- $\mathrm{N}=200$

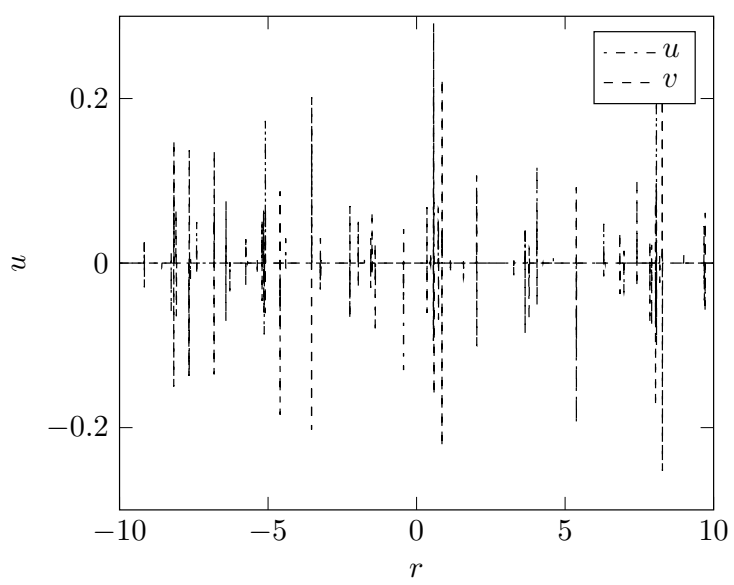

(c) velocity-fluctuations- $\mathrm{N}=400$

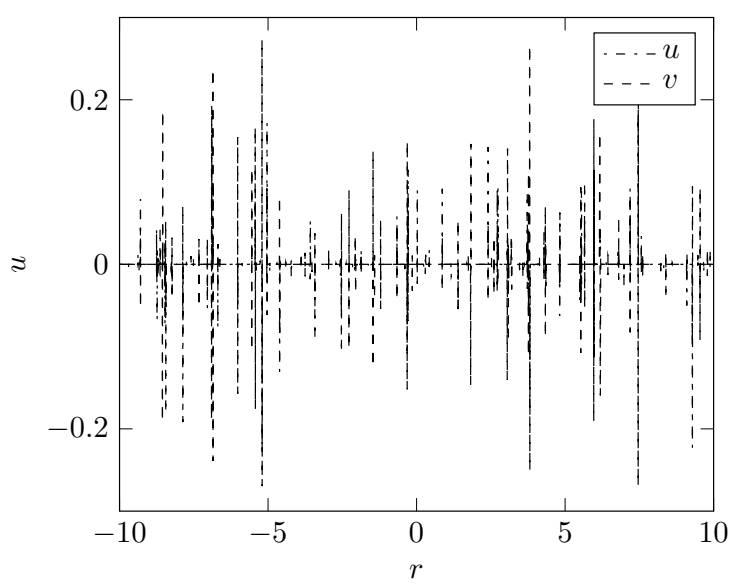

(e) velocity-fluctuations- $\mathrm{N}=800$

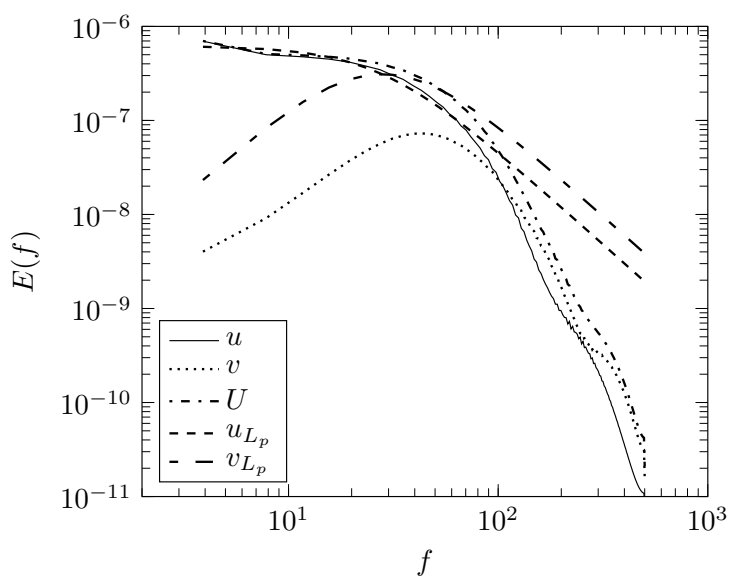

(b) spectra-Liepmann- $\mathrm{N}=200$

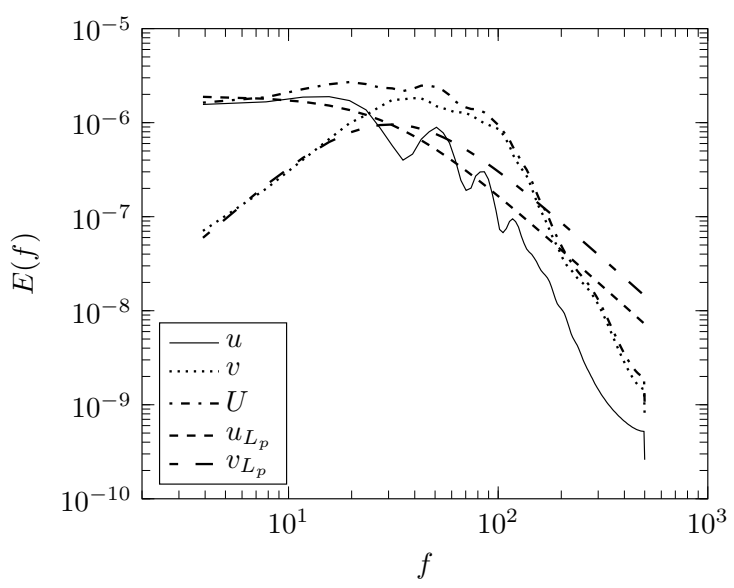

(d) spectra-Liepmann- $\mathrm{N}=400$

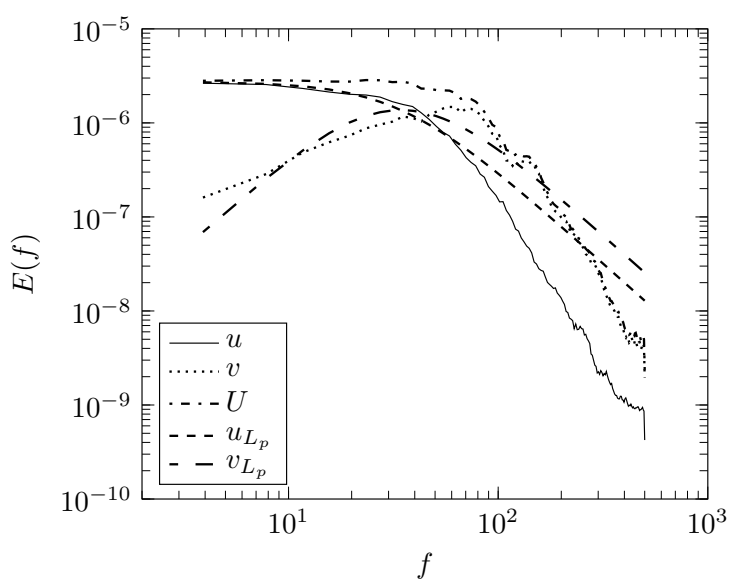

(f) spectra-Liepmann- $\mathrm{N}=800$

Figure 3.18: (l) Velocity fluctuations obtained at $(x, y)=(0,0)$, and (r) longitudinal and transverse energy spectra due to $(b) N=200,(d) N=400$, and $(e) N=800$ frozen Gaussian vortices arranged in $U U N R$ configuration convecting in the streamwise direction compared with Liepmann energy spectra of two-dimensional turbulence 


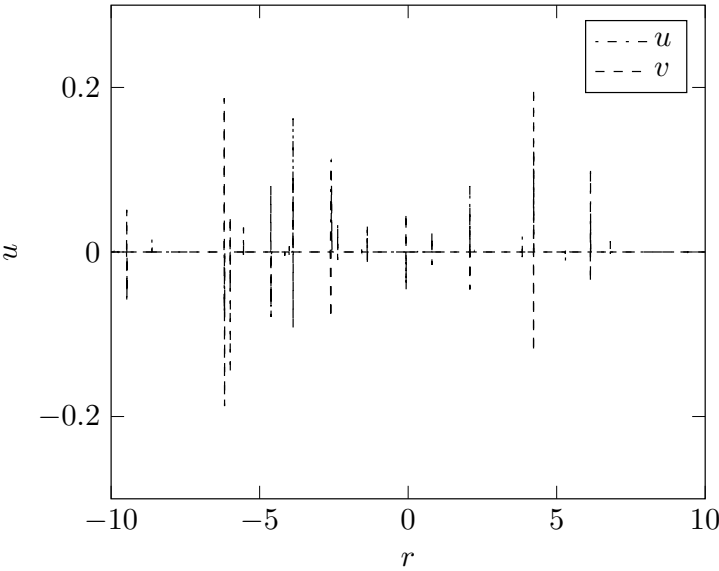

(a) velocity-fluctuations- $\mathrm{N}=200$

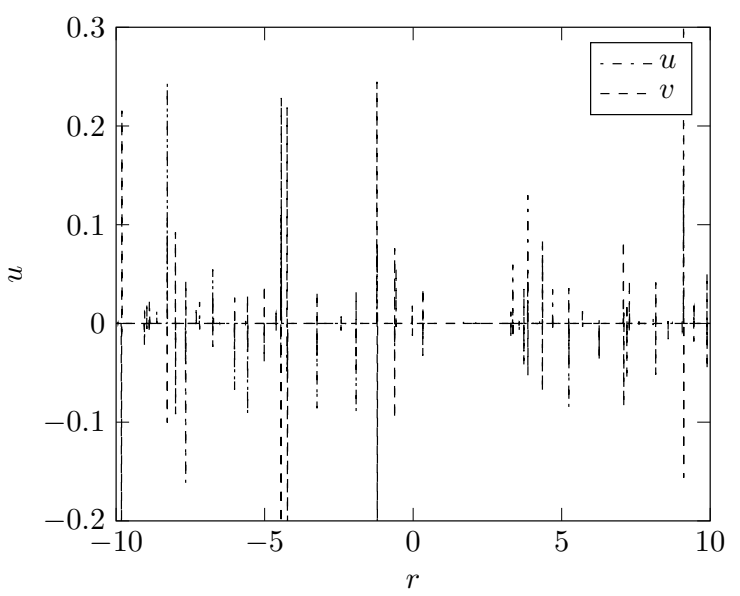

(c) velocity-fluctuations- $\mathrm{N}=400$

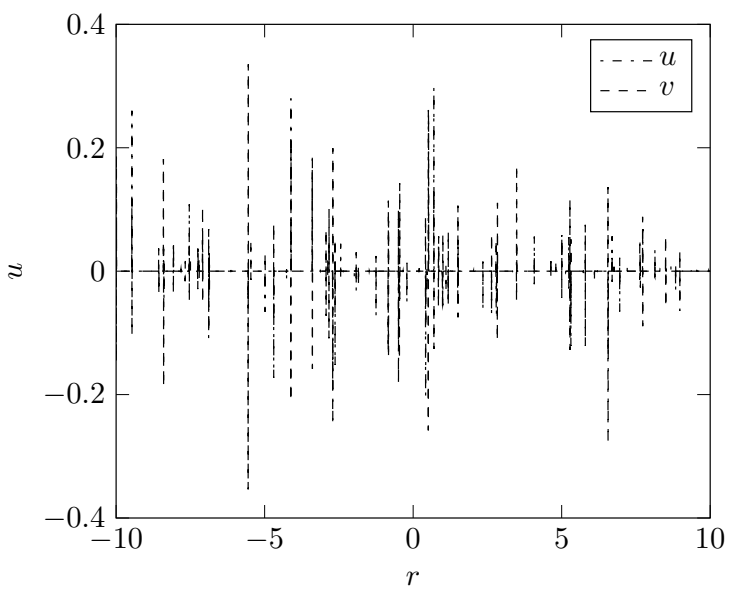

(e) velocity-fluctuations- $\mathrm{N}=800$

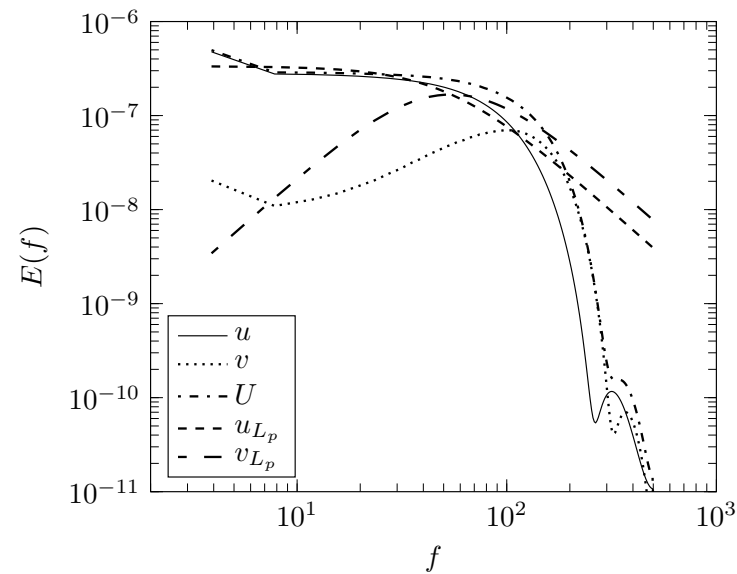

(b) spectra-Liepmann- $\mathrm{N}=200$

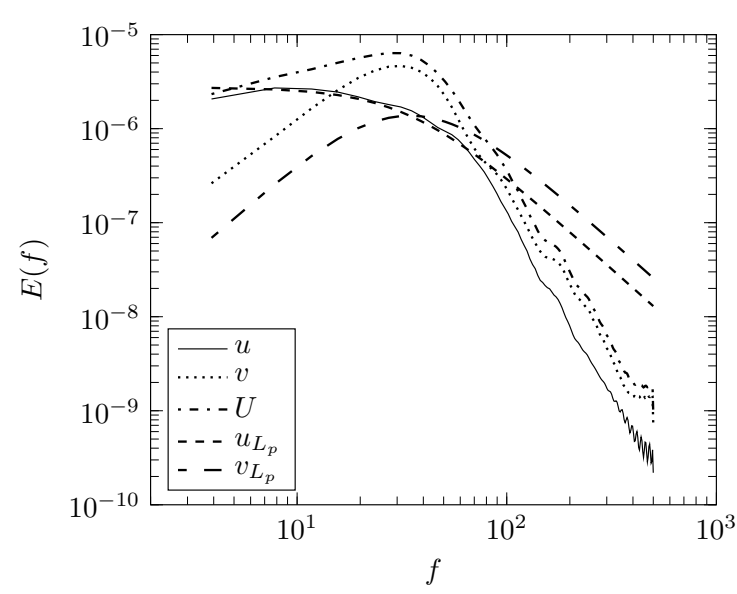

(d) spectra-Liepmann- $\mathrm{N}=400$

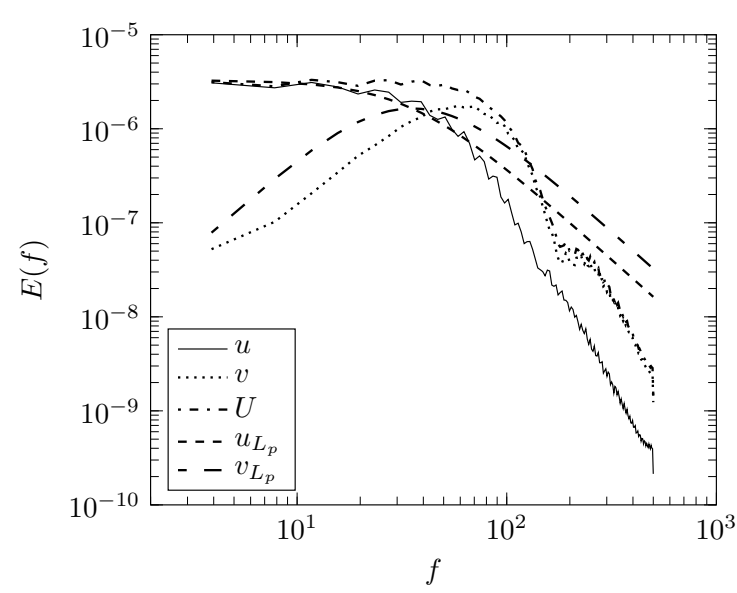

(f) spectra-Liepmann- $\mathrm{N}=800$

Figure 3.19: (l) Velocity fluctuations obtained at $(x, y)=(0,0)$, and (r) longitudinal and transverse energy spectra due to $(b) N=200,(d) N=400$, and $(e) N=800$ frozen Gaussian vortices arranged in $U N N R$ configuration convecting in the streamwise direction compared with Liepmann energy spectra of two-dimensional turbulence 


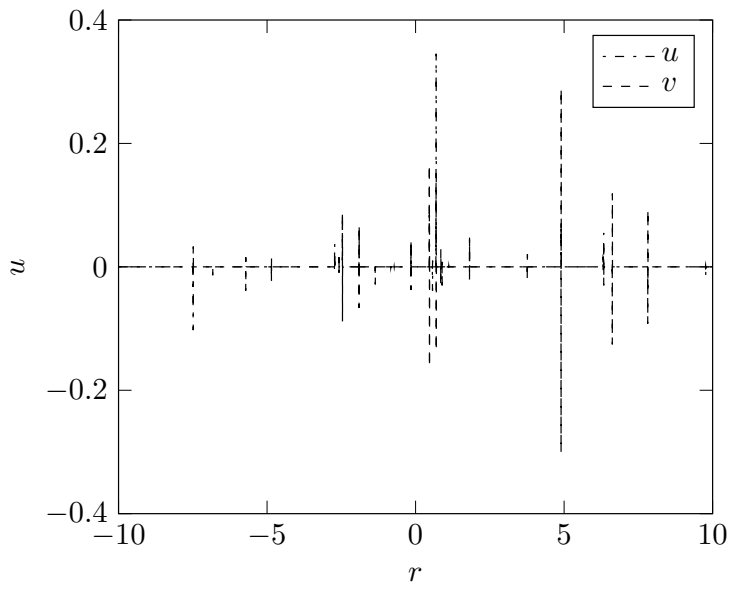

(a) velocity-fluctuations- $\mathrm{N}=200$

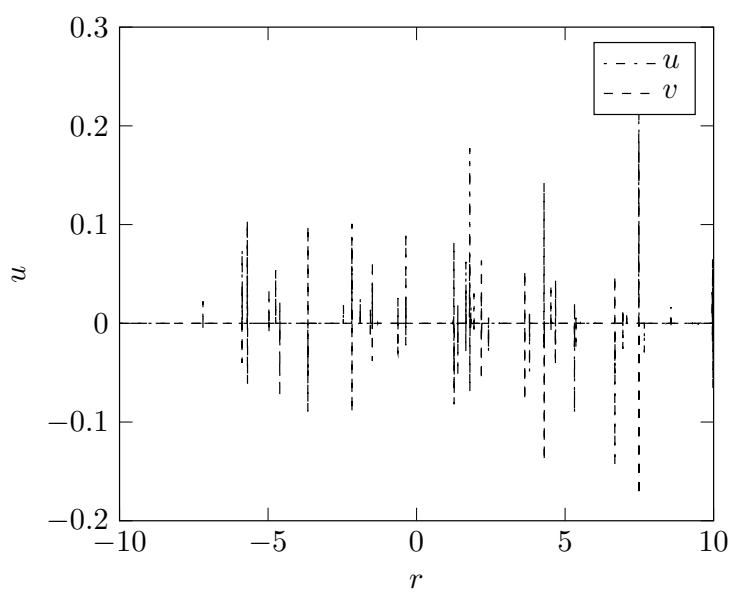

(c) velocity-fluctuations- $\mathrm{N}=400$

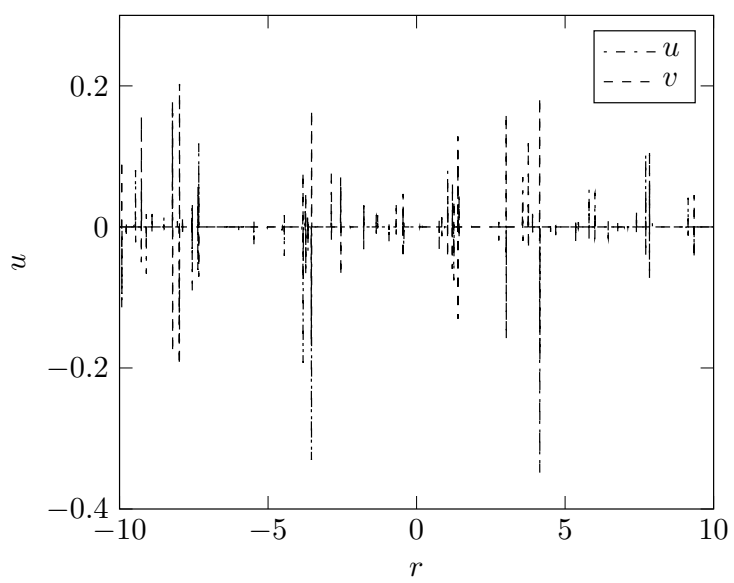

(e) velocity-fluctuations- $\mathrm{N}=800$

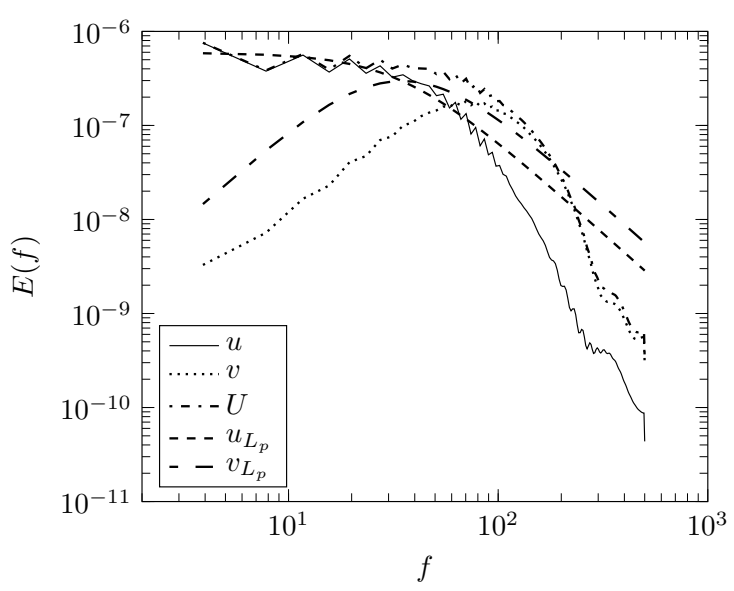

(b) spectra-Liepmann- $\mathrm{N}=200$

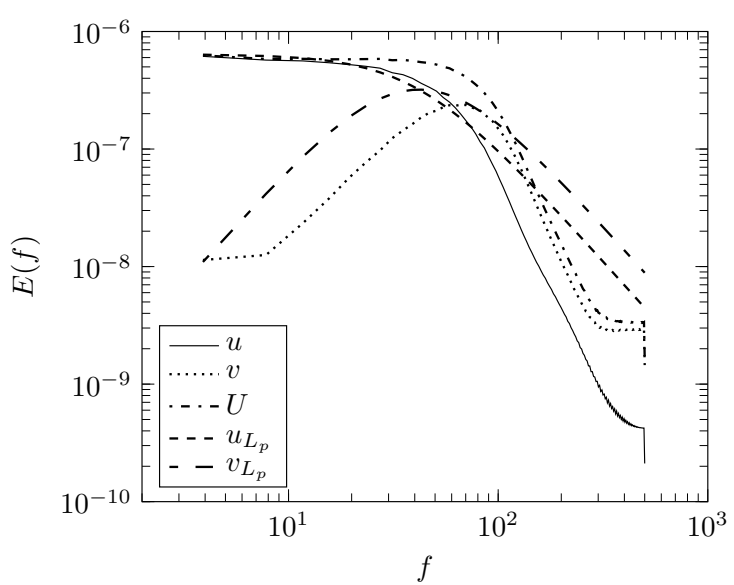

(d) spectra-Liepmann- $\mathrm{N}=400$

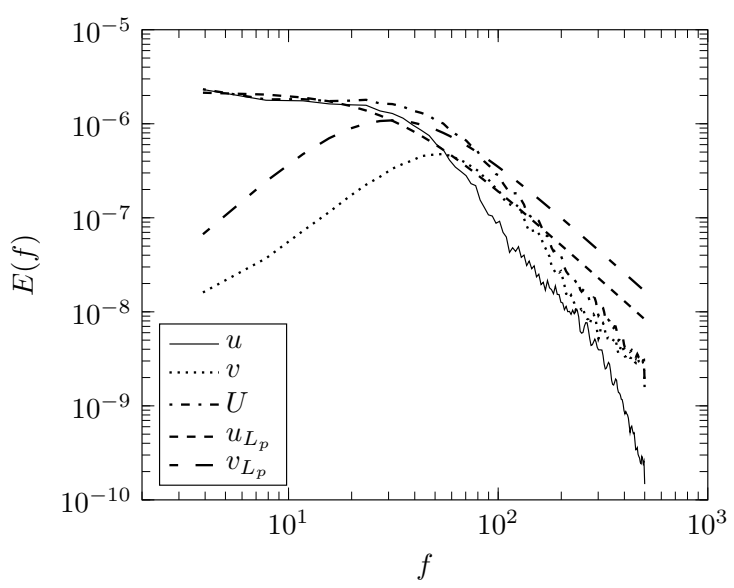

(f) spectra-Liepmann- $\mathrm{N}=800$

Figure 3.20: (l) Velocity fluctuations obtained at $(x, y)=(0,0)$, and (r) longitudinal and transverse energy spectra due to $(b) N=200,(d) N=400$, and $(e) N=800$ frozen Gaussian vortices arranged in NUNR configuration convecting in the streamwise direction compared with Liepmann energy spectra of two-dimensional turbulence 


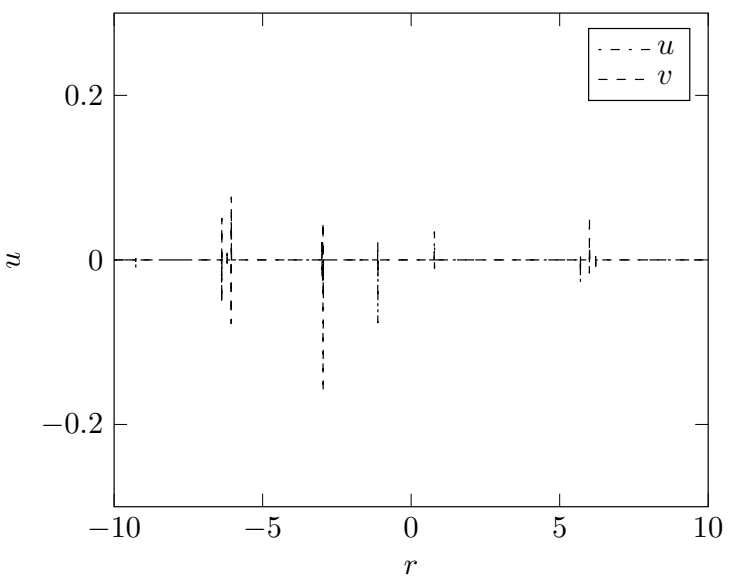

(a) velocity-fluctuations- $\mathrm{N}=200$

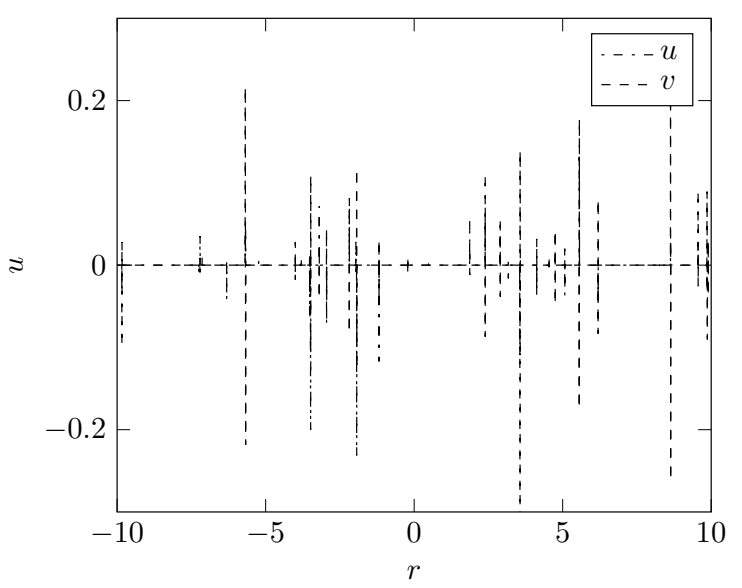

(c) velocity-fluctuations- $\mathrm{N}=400$

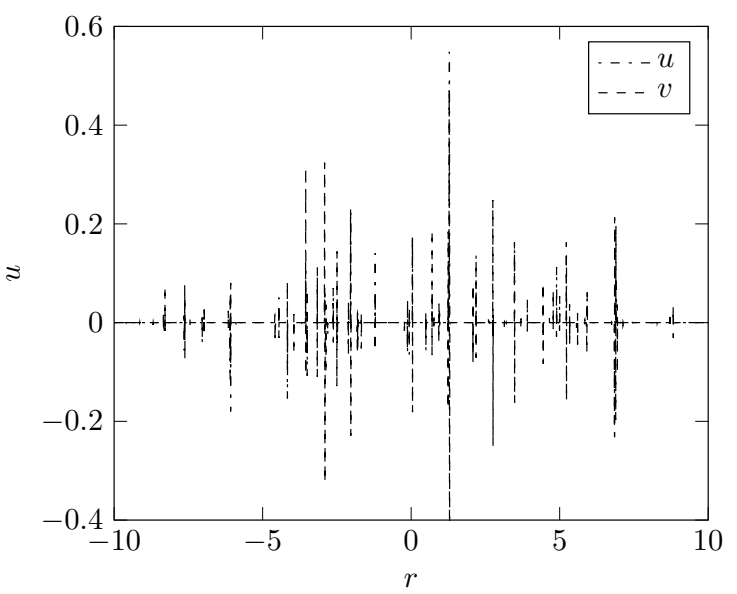

(e) velocity-fluctuations- $\mathrm{N}=800$

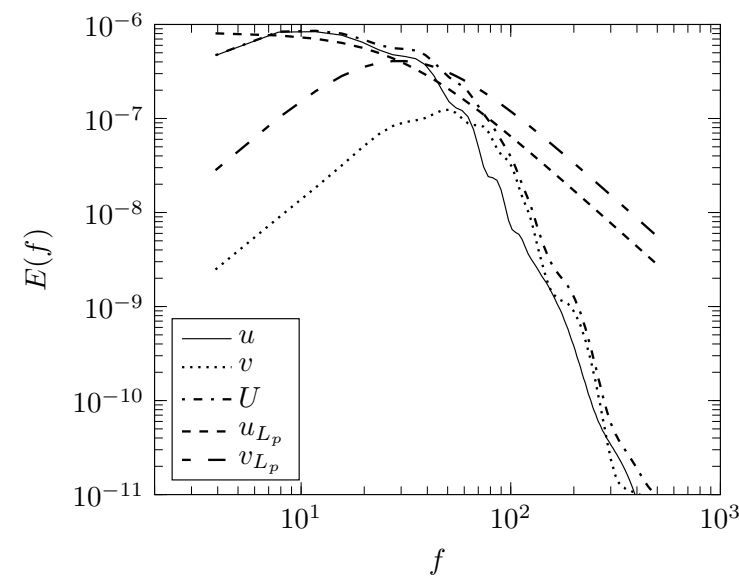

(b) spectra-Liepmann- $\mathrm{N}=200$

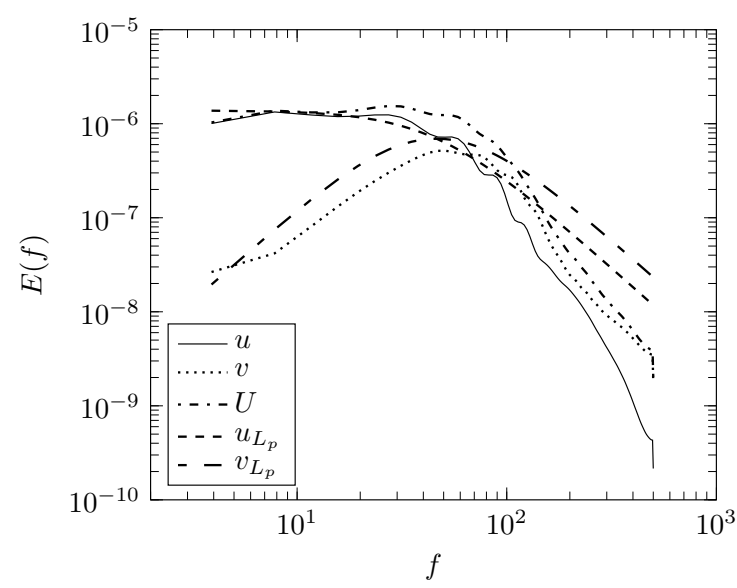

(d) spectra-Liepmann- $\mathrm{N}=400$

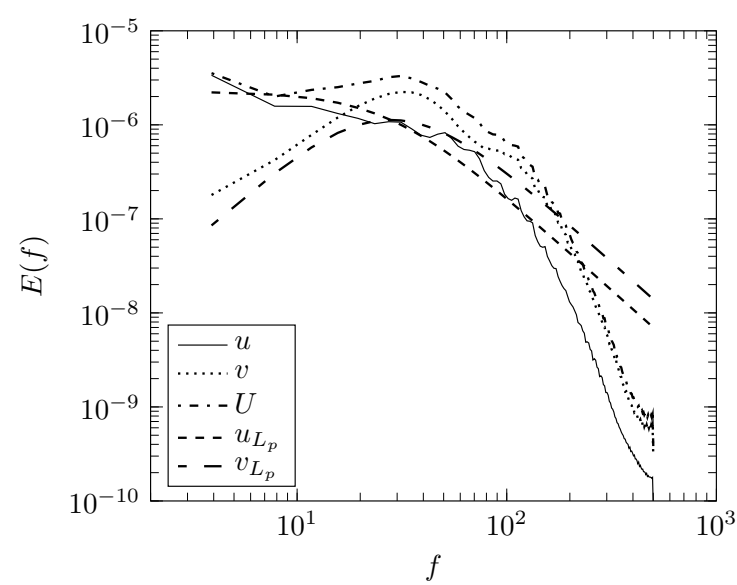

(f) spectra-Liepmann- $\mathrm{N}=800$

Figure 3.21: (l) Velocity fluctuations obtained at $(x, y)=(0,0)$, and (r) longitudinal and transverse energy spectra due to $(b) N=200,(d) N=400$, and $(e) N=800$ frozen Gaussian vortices arranged in $N N N R$ configuration convecting in the streamwise direction compared with Liepmann energy spectra of two-dimensional turbulence 


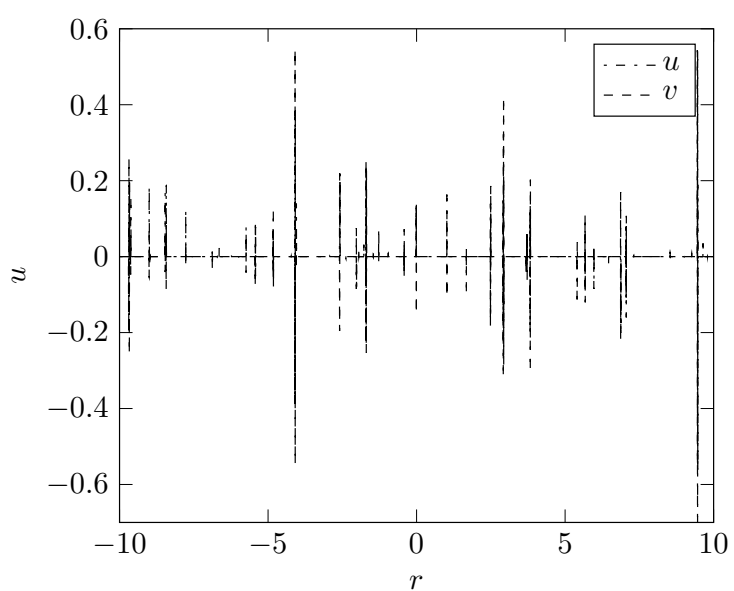

(a) velocity-fluctuations- $\mathrm{N}=200$

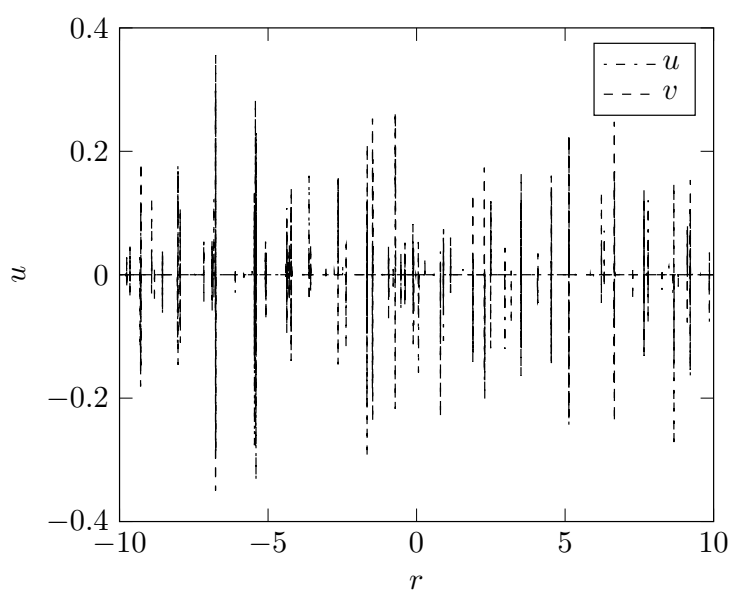

(c) velocity-fluctuations- $\mathrm{N}=400$

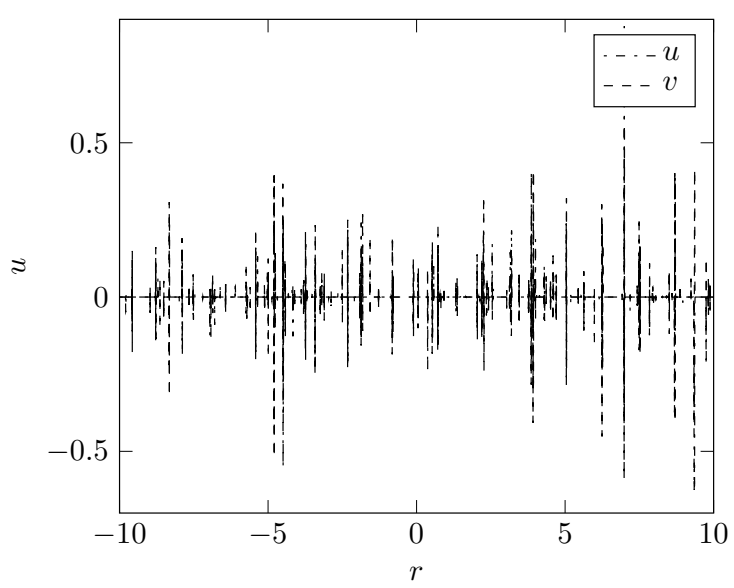

(e) velocity-fluctuations- $\mathrm{N}=800$

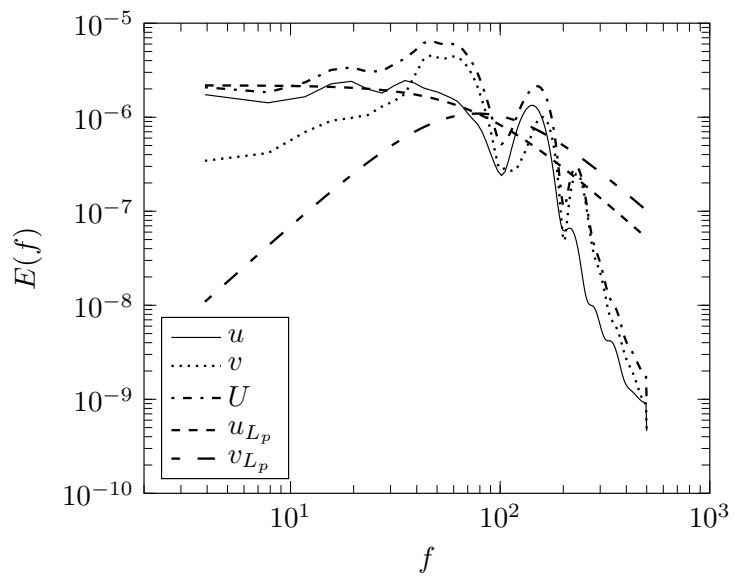

(b) spectra-Liepmann- $\mathrm{N}=200$

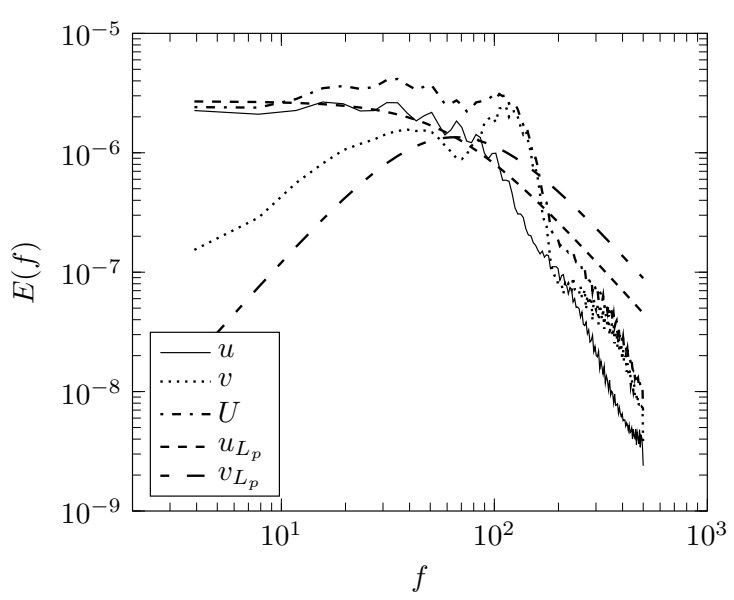

(d) spectra-Liepmann- $\mathrm{N}=400$

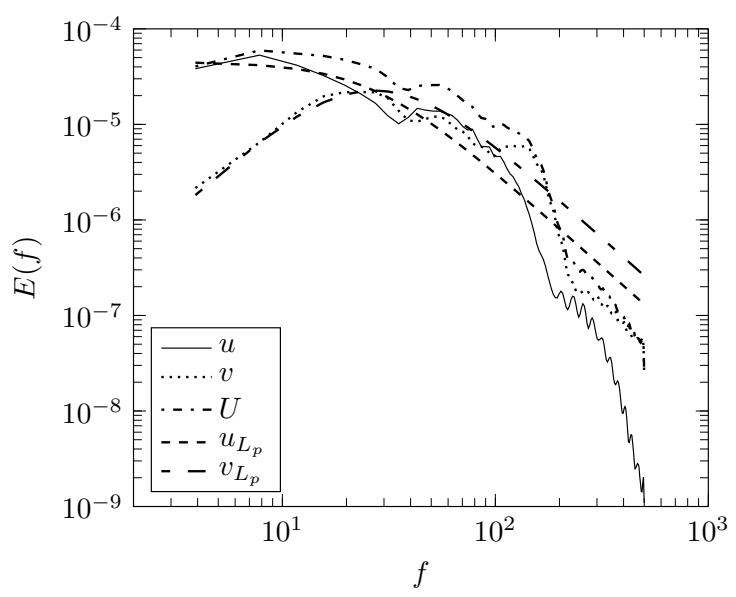

(f) spectra-Liepmann- $\mathrm{N}=800$

Figure 3.22: (l) Velocity fluctuations obtained at $(x, y)=(0,0)$, and $(\mathrm{r})$ longitudinal and transverse energy spectra due to $(b) N=200,(d) N=400$, and $(e) N=800$ frozen Mexican-hat vortices arranged in $U U N R$ configuration convecting in the streamwise direction compared with Liepmann energy spectra of two-dimensional turbulence 


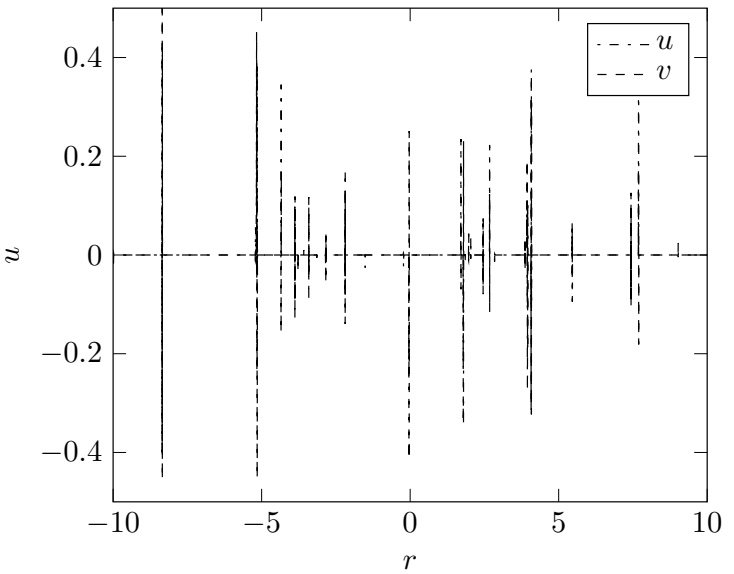

(a) velocity-fluctuations- $\mathrm{N}=200$

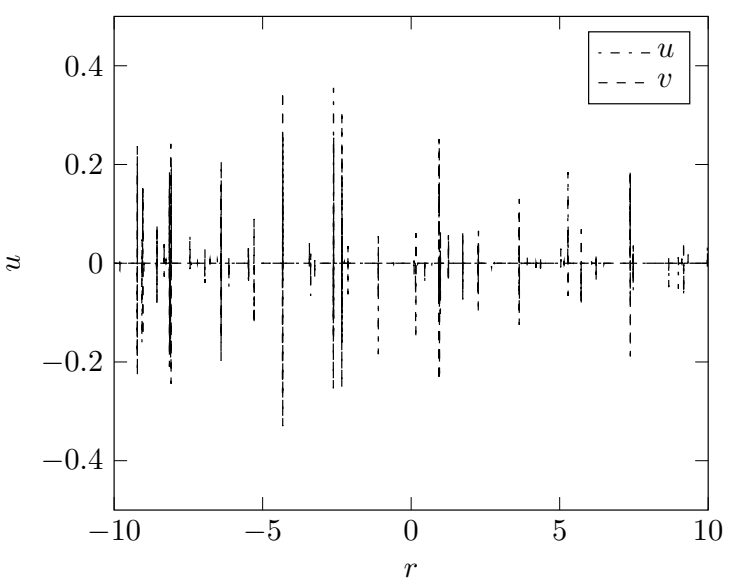

(c) velocity-fluctuations- $\mathrm{N}=400$

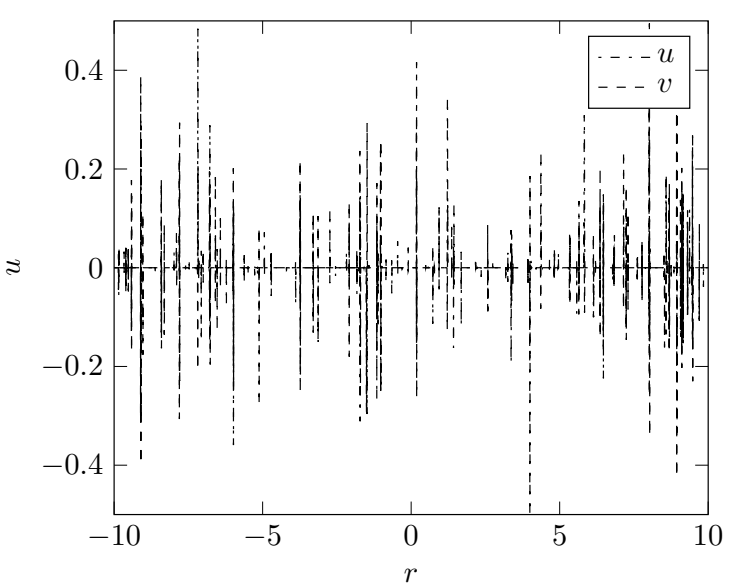

(e) velocity-fluctuations- $\mathrm{N}=800$

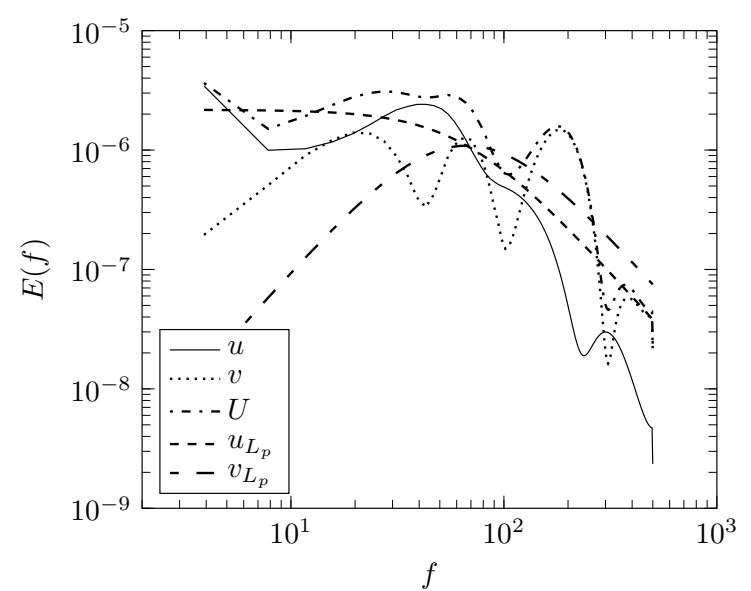

(b) spectra-Liepmann- $\mathrm{N}=200$

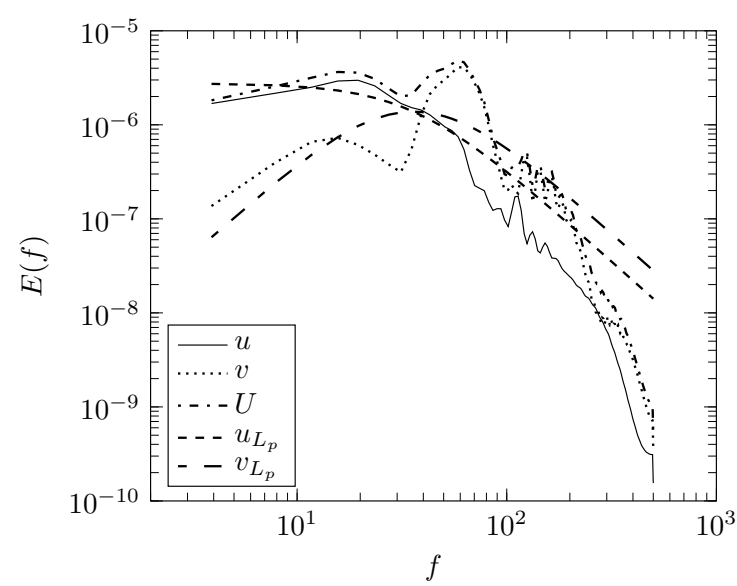

(d) spectra-Liepmann- $\mathrm{N}=400$

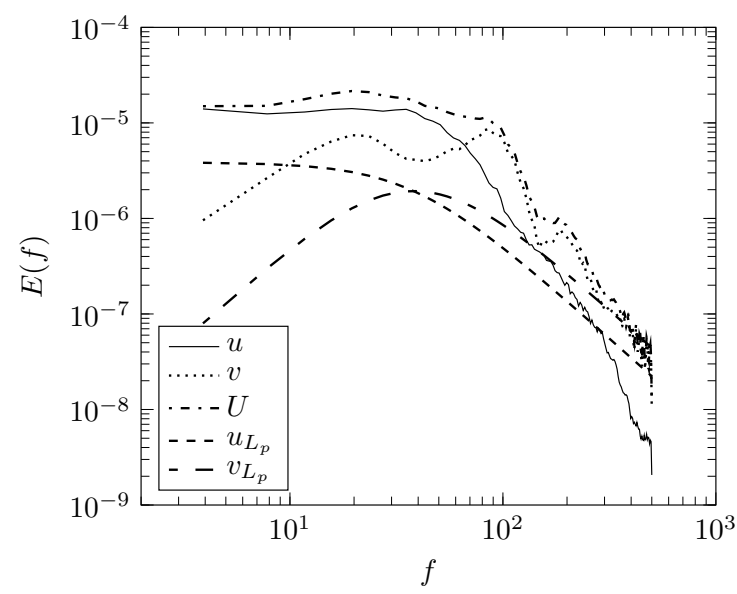

(f) spectra-Liepmann- $\mathrm{N}=800$

Figure 3.23: (l) Velocity fluctuations obtained at $(x, y)=(0,0)$, and (r) longitudinal and transverse energy spectra due to $(b) N=200,(d) N=400$, and $(e) N=800$ frozen Mexican-hat vortices arranged in $U N N R$ configuration convecting in the streamwise direction compared with Liepmann energy spectra of two-dimensional turbulence 


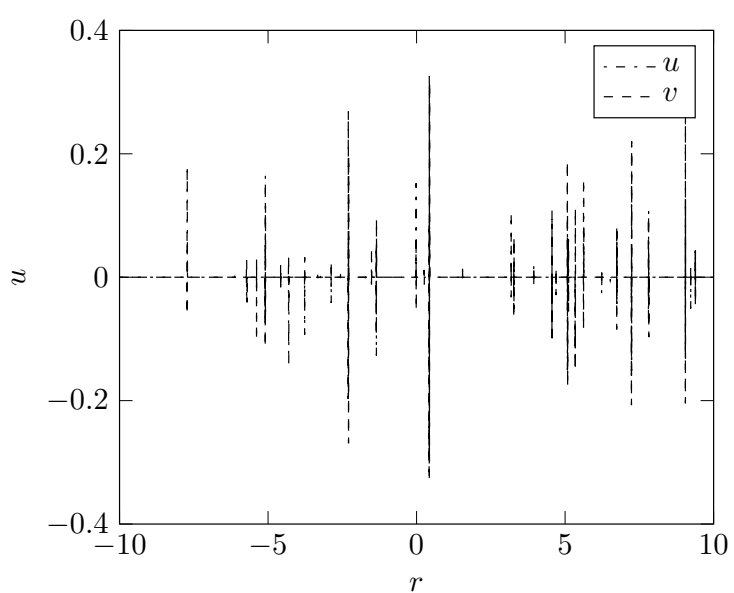

(a) velocity-fluctuations- $\mathrm{N}=200$

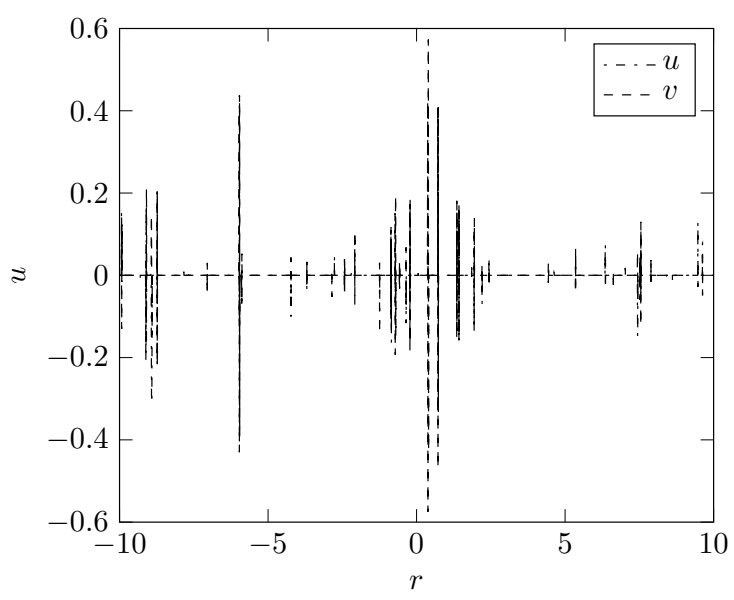

(c) velocity-fluctuations- $\mathrm{N}=400$

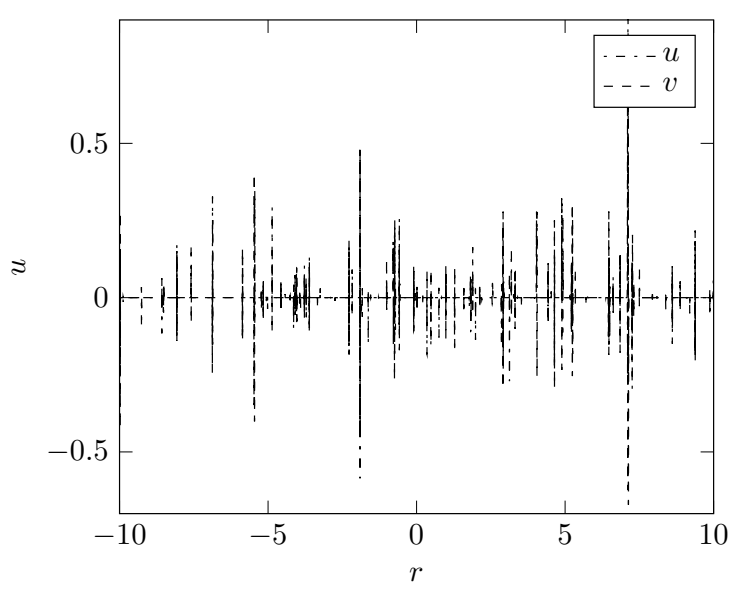

(e) velocity-fluctuations- $\mathrm{N}=800$

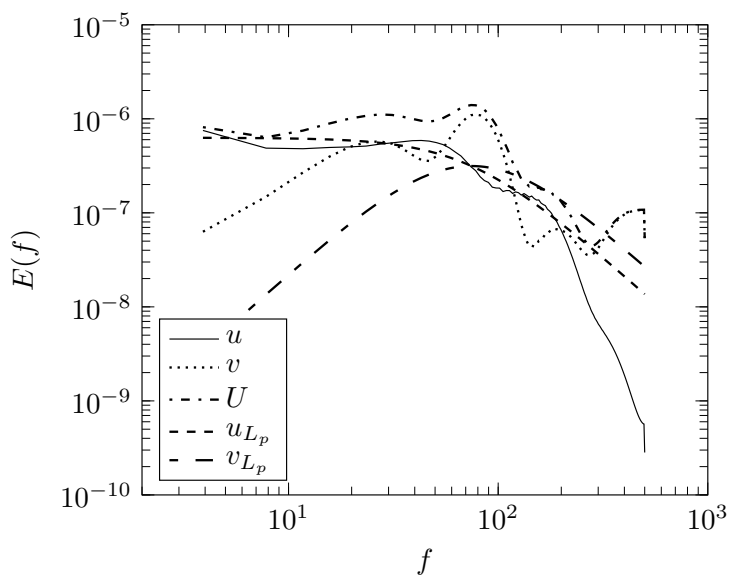

(b) spectra-Liepmann- $\mathrm{N}=200$

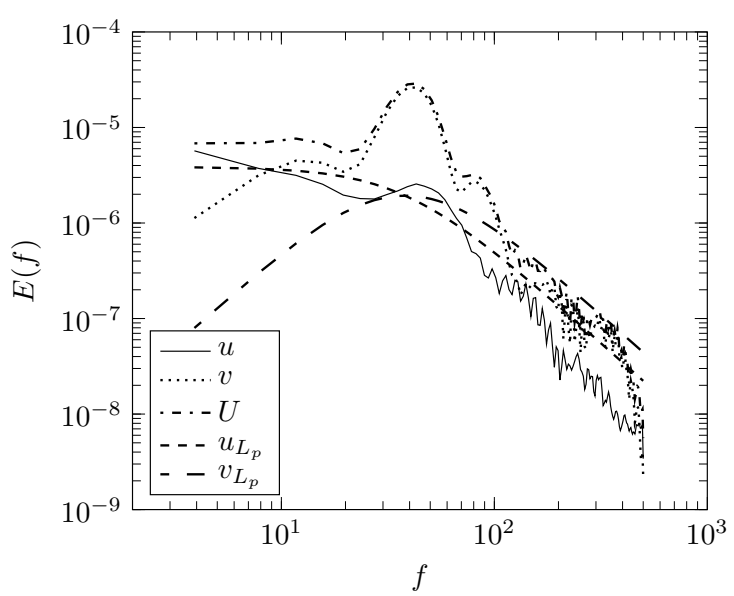

(d) spectra-Liepmann- $\mathrm{N}=400$

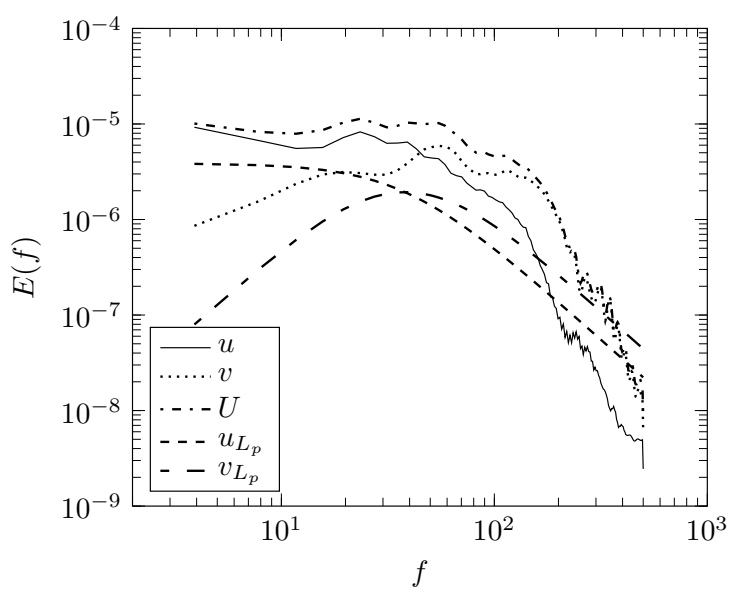

(f) spectra-Liepmann- $\mathrm{N}=800$

Figure 3.24: (l) Velocity fluctuations obtained at $(x, y)=(0,0)$, and $(\mathrm{r})$ longitudinal and transverse energy spectra due to $(b) N=200,(d) N=400$, and $(e) N=800$ frozen Mexican-hat vortices arranged in NUNR configuration convecting in the streamwise direction compared with Liepmann energy spectra of two-dimensional turbulence 


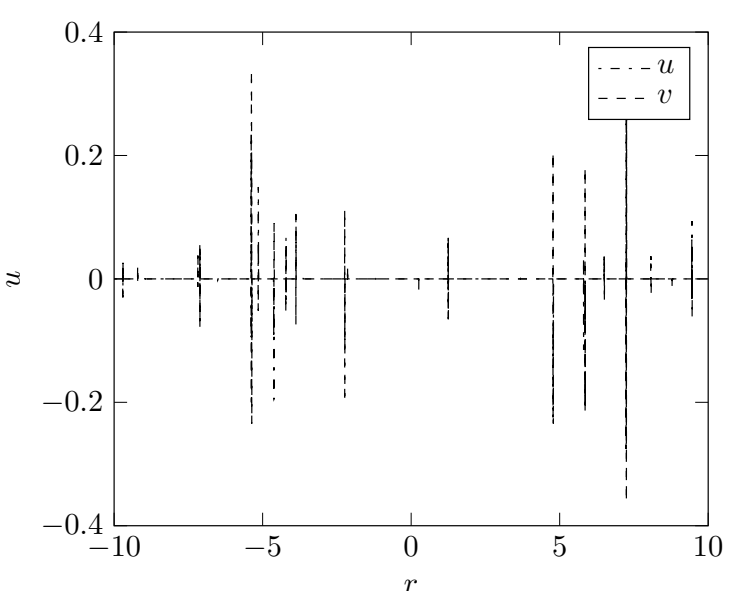

(a) velocity-fluctuations- $\mathrm{N}=200$

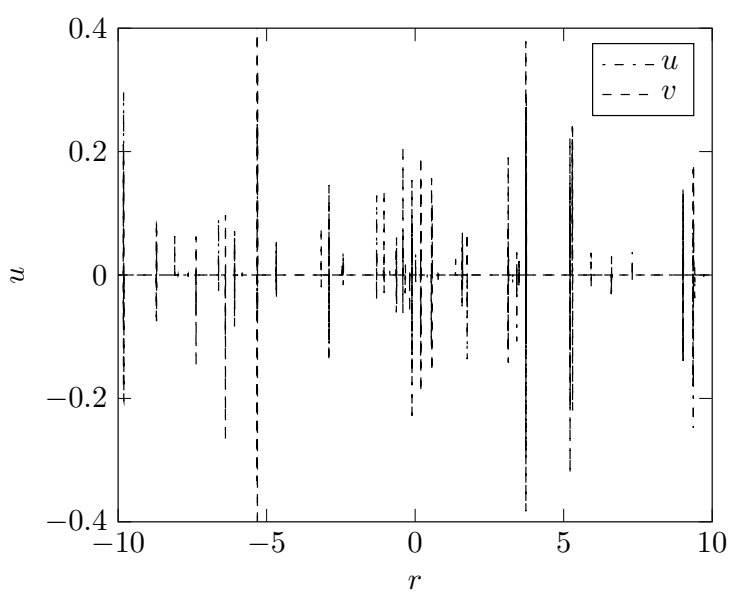

(c) velocity-fluctuations- $\mathrm{N}=400$

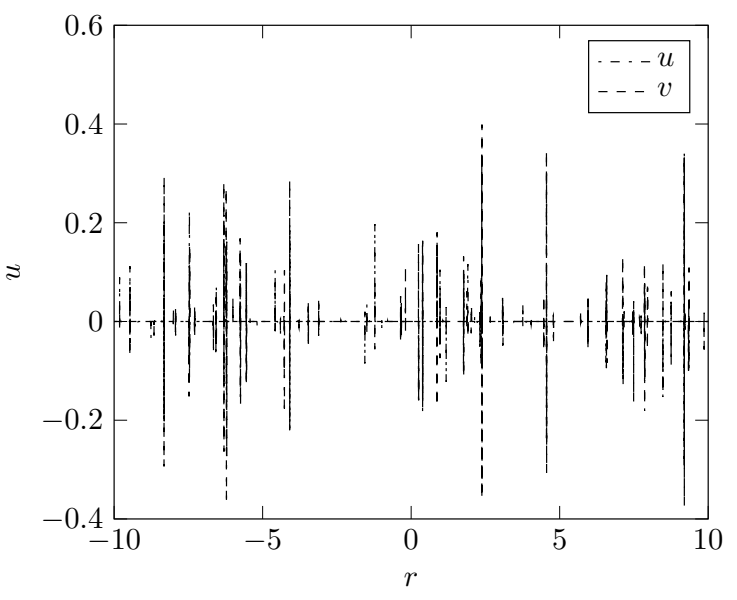

(e) velocity-fluctuations- $\mathrm{N}=800$

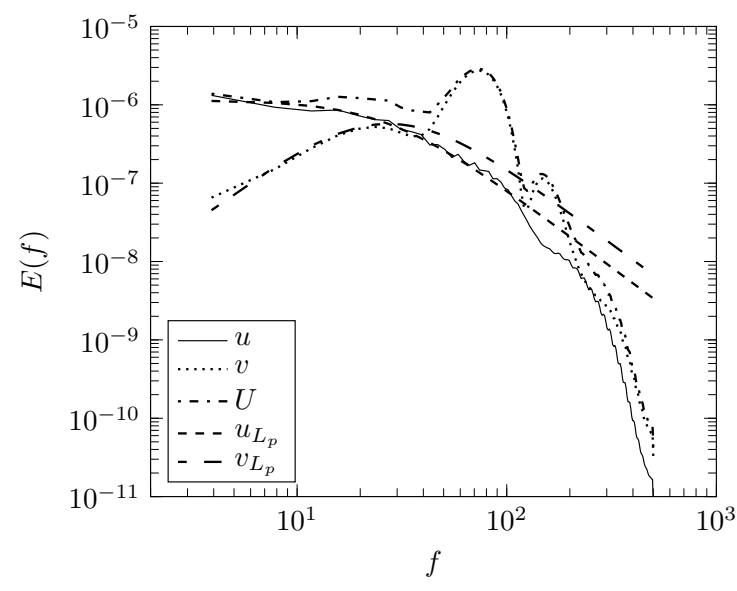

(b) spectra-Liepmann- $\mathrm{N}=200$

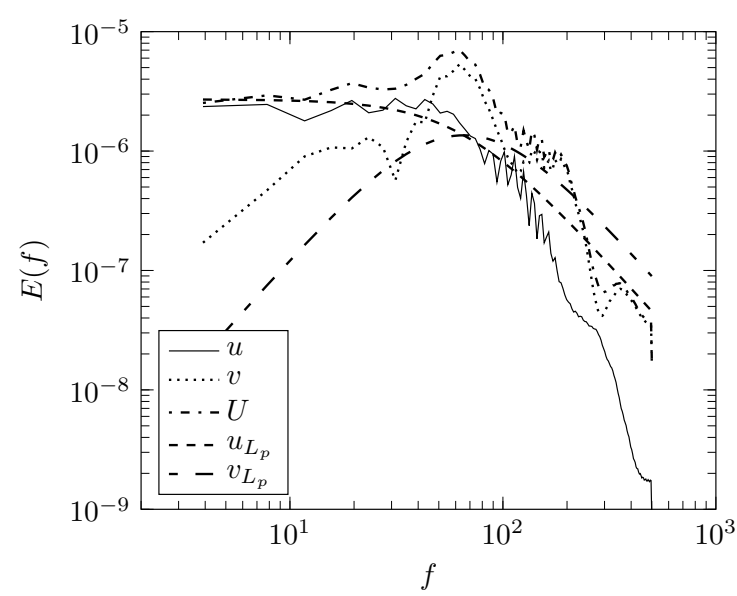

(d) spectra-Liepmann- $\mathrm{N}=400$

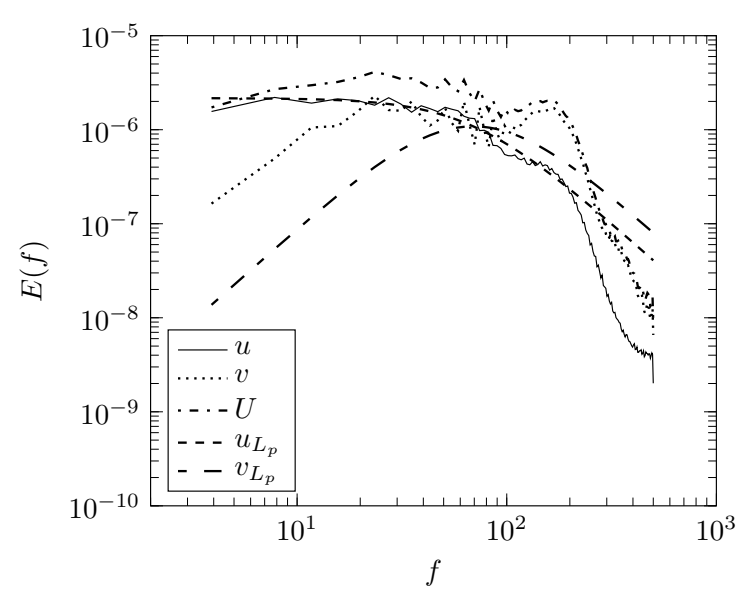

(f) spectra-Liepmann- $\mathrm{N}=800$

Figure 3.25: (l) Velocity fluctuations obtained at $(x, y)=(0,0)$, and $(\mathrm{r})$ longitudinal and transverse energy spectra due to $(b) N=200,(d) N=400$, and $(e) N=800$ frozen Mexican-hat vortices arranged in $N N N R$ configuration convecting in the streamwise direction compared with Liepmann energy spectra of two-dimensional turbulence 
Table 3.6: Association between vortices parameters and turbulence parameters; $\left(\mathrm{u}_{r m s}, \lambda\right) \leftrightarrow(\Gamma$, $R$ )

\begin{tabular}{lllcl}
\hline Vortex & $\Gamma_{r m s}$ & $R_{\text {scale }}$ & $u_{\text {rms }}^{\prime} / u$ & $\lambda$ \\
\hline \multirow{2}{*}{ Mexican- } & 0.1 & 0.1 & 0.0119 & 0.0042 \\
hat & 0.4 & 0.1 & 0.049 & 0.0042 \\
& 1.0 & 0.1 & 0.132 & 0.0055 \\
Gaussian & 0.1 & 0.1 & 0.00652 & 0.00716 \\
& 0.4 & 0.1 & - & - \\
& 1.0 & 0.1 & - & - \\
\hline
\end{tabular}

of vortex strength distribution, $\Gamma_{r m s}$. It is essential to know the relationship between the control parameters of the vortex and the turbulence for further aeroacoustic investigation in the next chapter.

Using the above algorithm for the optimisation of velocity spectral density, two main cases are investigated, one for Mexican-hat mollified vortices, and another for the Gaussian mollified vortices. The vortices are confined in a vortex window of $100 c_{a}$ length and $0.2 c_{a}$ width. From the earlier section, $f_{v p}$ is fixed at 110 , which gives 10000 vortices in the imaginary computational domain which is convecting in the streamwise direction with $U=5 \mathrm{~m} / \mathrm{s}$. The vortices are distributed in uniform distribution across the window, and vortex strength and radius are employing the normal and Rayleigh distributions, respectively. The values of $N$ and $A$ are fixed which allow the room for variations in $\Gamma$ and $R$. By keeping the Rayleigh scale parameter constant equal to 0.1 for $p(R)$, standard deviation or $\Gamma_{r m s}$ for the normal distribution of $p(\Gamma)$ is varied as $\Gamma_{r m s} \in\{0.1,0.4,1.0\}$. The optimisation is carried out for 300 different random distributions for the vortex parameters. Fig. (3.26) shows a very good agreement between the spectral densities of the longitudinal and the transverse velocity components with the corresponding target spectral densities of Liepmann spectra for two-dimensional turbulence. The corresponding values of $u_{r m s}$ and $\lambda$ of the fluctuating velocity field induced by the vortex window configured with $U U N R$ distribution with different values of $\Gamma_{r m s}$ which are tabulated in the Table. (3.6). The configurations generated the same set of turbulence characteristics for 278 sets of distribution which gives the success rate of $92.67 \%$. Similarly, three cases are built with Gaussian vortices with the same values of $\Gamma_{r m s}$ and Rayleigh scale parameter as for the Mexican-hat vortices. It is found that with $\Gamma_{r m s}=\{0.4,1.0\}$, the velocity field generated by Gaussian vortices do not get optimised with the target curve of Liepmann spectra; however, the turbulent velocity field induced by using $\Gamma_{r m s}=0.1$ is very weak. This can be understood from the results shown in sec. (‥6.6.1), which show that the field induced by Gaussian vortices quickly dissipates energy.

Here one can simply argue to convect the vortices arranged in a specific configuration in vortex window with desired statistical characteristics around/over the airfoil, and then record the time-history of the induced velocity fluctuations to calculate the pressure fluctuations as input for aeroacoustic simulation further. But as mentioned earlier in the chapter, about the memory requirements because the vortex particle methods require the evaluation of an N-body interaction problem at each iteration. The particles convect in a Lagrangian fashion 
in which their positions change at each time-step which essentially require the re-computation of particle-particle influences. Due to this, the model proposed can not be simply convected and allow to influence and get influenced by other particles.

This is the subject of the next chapter where this vortex statistical method has been moulded for aeroacoustic calculations. 


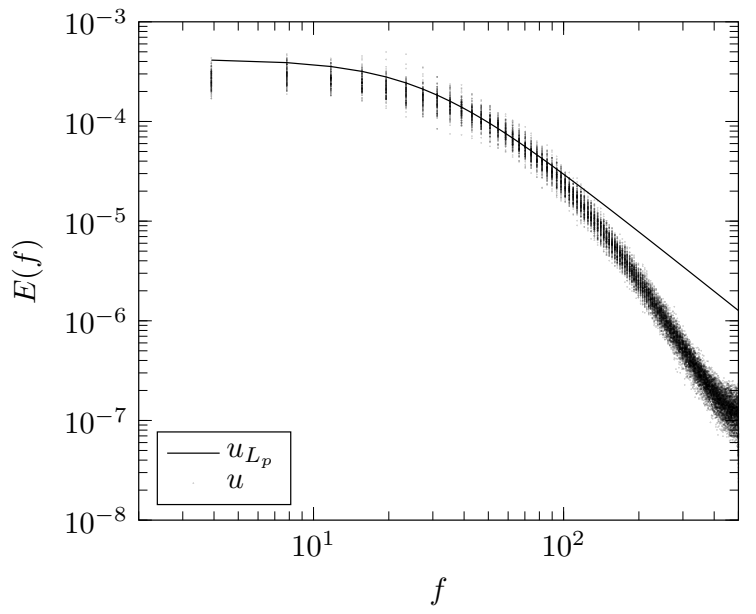

(a) longitudinal spectrum $u_{r m s} / U=0.132, \lambda=$ $0.0055, N=10000$, Mexican - hat,$\Gamma_{r m s}=$ $1.0, \rho_{r m s}=0.1$

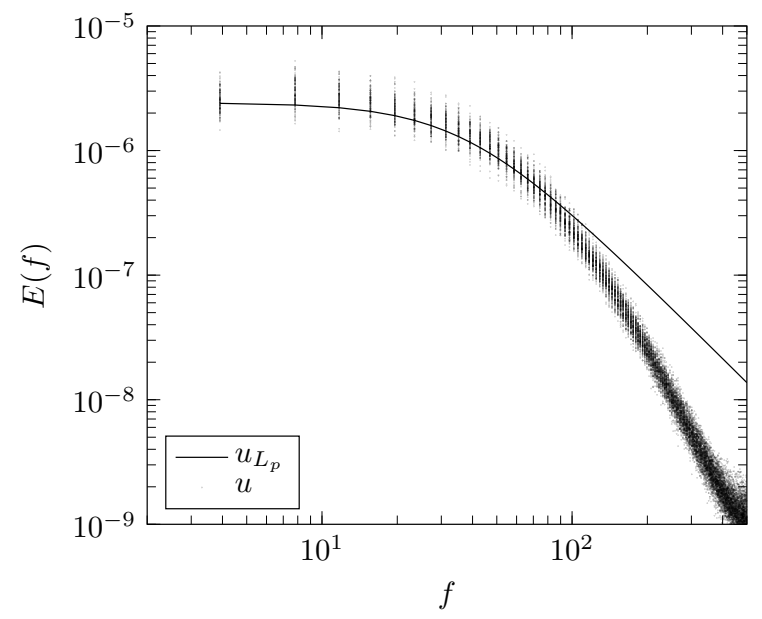

(c) longitudinal spectrum $u_{r m s} / U=0.0119, \lambda=$ $0.00421, N=10000$, Mexican - hat,$\Gamma_{r m s}=$ $0.1, \rho_{r m s}=0.1$

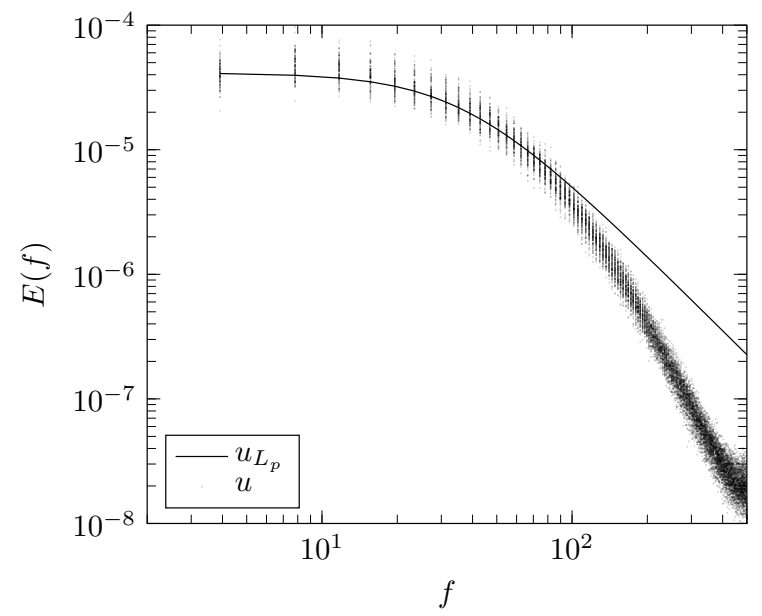

(e) longitudinal spectrum $u_{r m s} / U=0.049, \lambda=$ $0.00429, N=10000$, Mexican - hat,$\Gamma_{r m s}=$ $0.4, \rho_{r m s}=0.1$

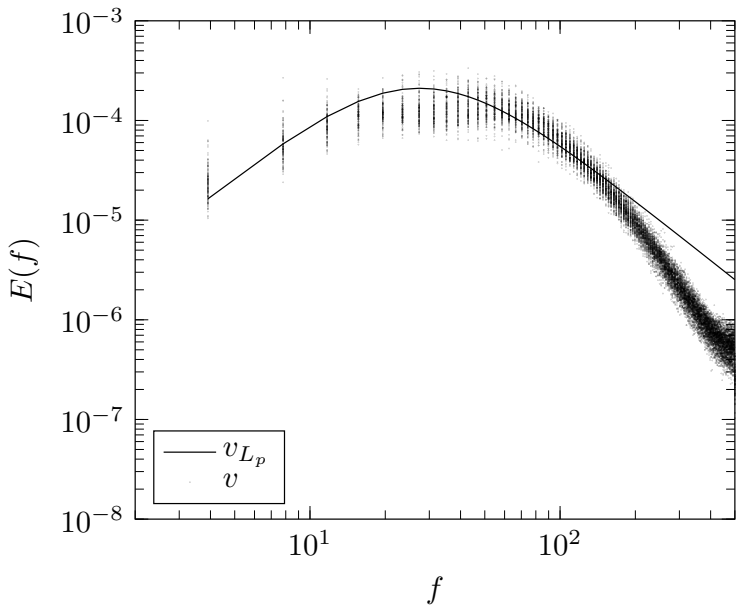

(b) transverse spectrum $u_{r m s} / U=0.132, \lambda=$ $0.0055, N=10000$, Mexican - hat,$\Gamma_{r m s}=$ $1.0, \rho_{r m s}=0.1$

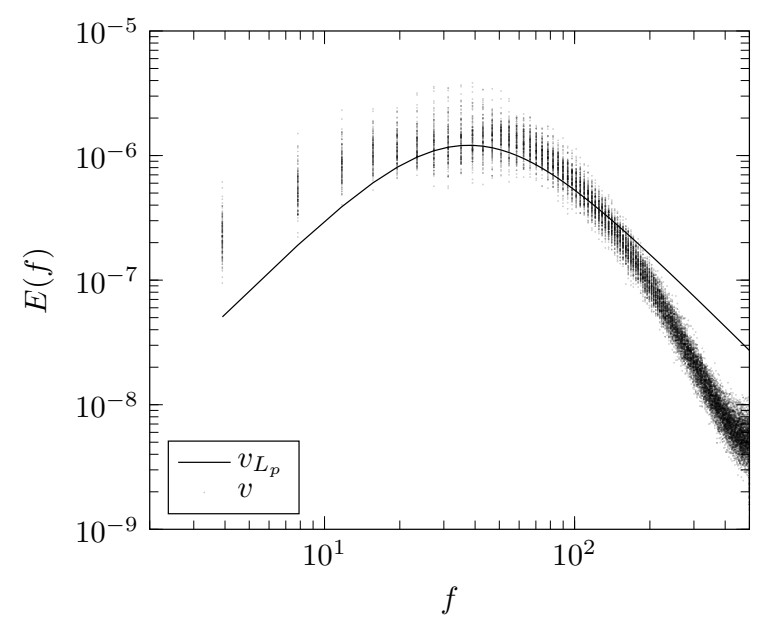

(d) transverse spectrum $u_{r m s} / U=0.0119, \lambda=$ $0.00421, N=10000$, Mexican - hat,$\Gamma_{r m s}=$ $0.1, \rho_{\text {rms }}=0.1$

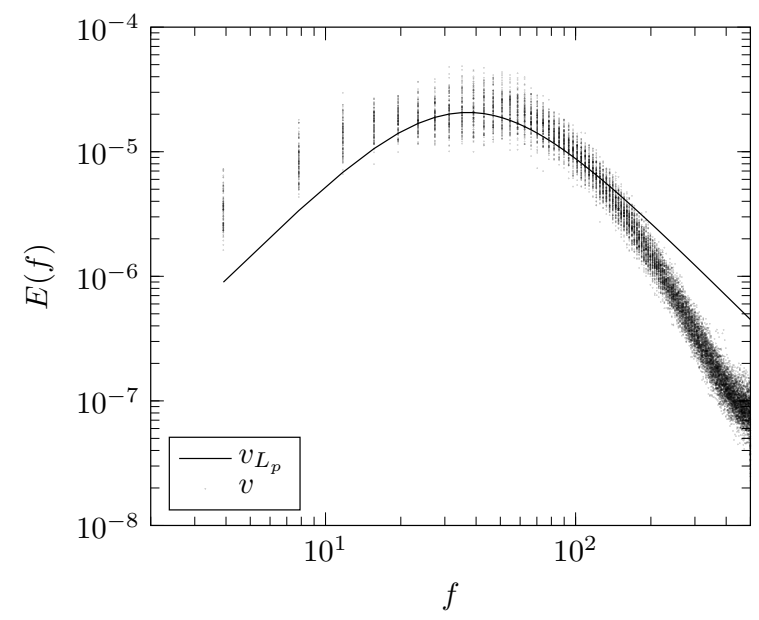

(f) transverse spectrum $u_{r m s} / U=0.049, \lambda=$ $0.00429, N=10000$, Mexican - hat, $\Gamma_{r m s}=$ $0.4, \rho_{r m s}=0.1$

Figure 3.26: 300 iterations per case, Liepmann spectra; Mexican-hat, $u_{r m s}$-lamda-gamma-radiusassociation 


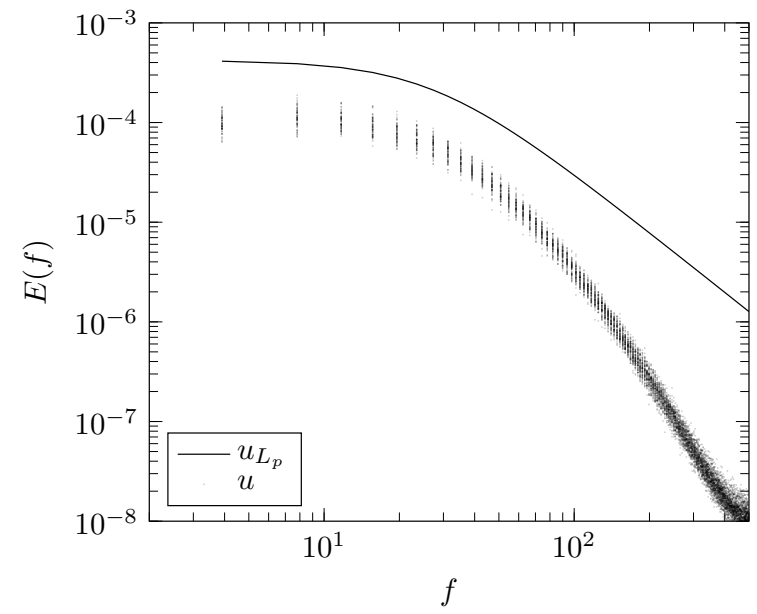

(a) longitudinal spectrum $u_{r m s} / U=0.132, \lambda=$ $0.0055, N=10000$, Gaussian, $\Gamma_{r m s}=1.0, \rho_{r m s}=0.1$

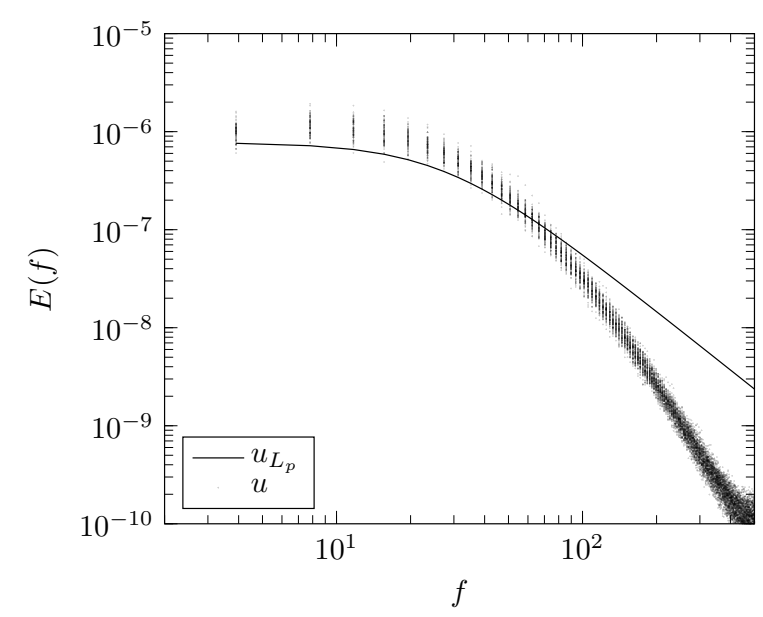

(c) longitudinal spectrum $u_{r m s} / U=0.00652, \lambda=$ $0.00716, N=10000$, Gaussian, $\Gamma_{r m s}=0.1, \rho_{r m s}=$ 0.1

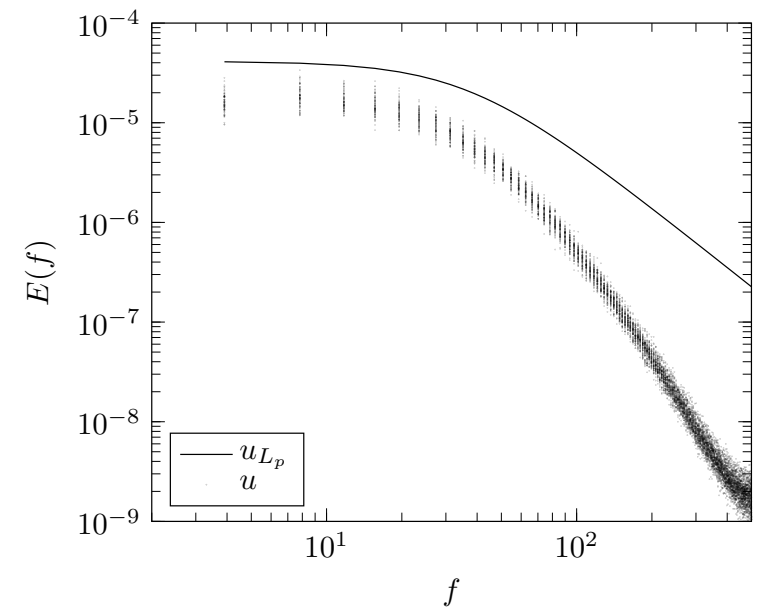

(e) longitudinal spectrum $u_{r m s} / U=0.049, \lambda=$ $0.00429, N=10000$, Gaussian $, \Gamma_{r m s}=0.4, \rho_{r m s}=$ 0.1

Figure 3.27: 300 iterations per case, Liepmann spectra; Gaussian, $u_{r m s}$-lamda-gamma-radiusassociation

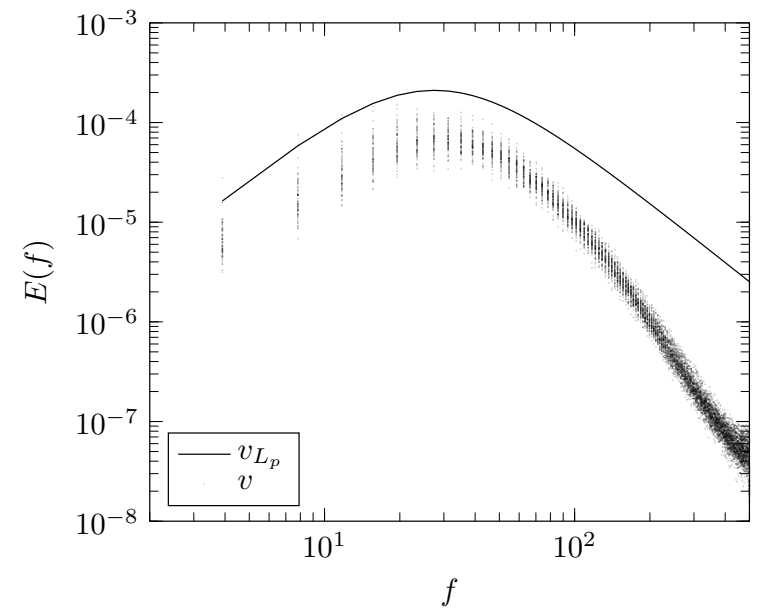

(b) transverse spectrum $u_{r m s} / U=0.132, \lambda=$ $0.0055, N=10000$, Gaussian, $\Gamma_{r m s}=1.0, \rho_{r m s}=0.1$

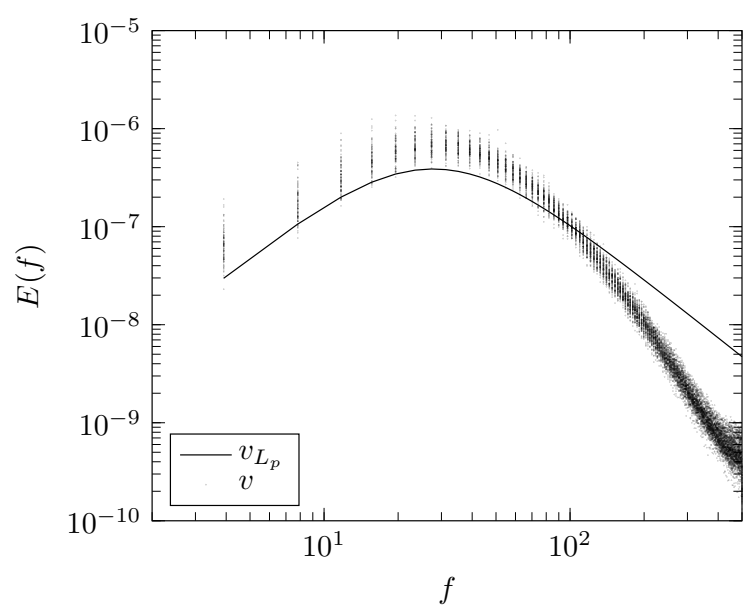

(d) transverse spectrum $u_{r m s} / U=0.00652, \lambda=$ $0.00716, N=10000$, Gaussian $, \Gamma_{r m s}=0.1, \rho_{r m s}=$ 0.1

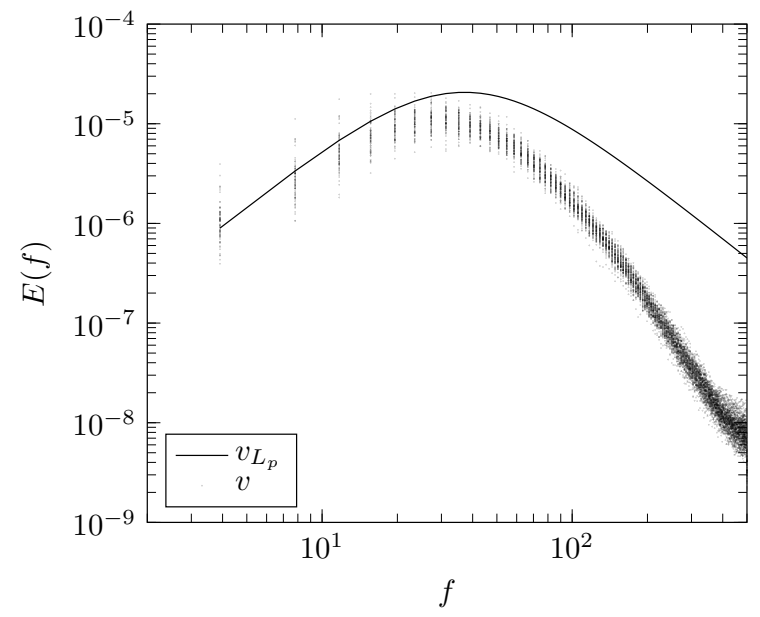

(f) transverse spectrum $u_{r m s} / U=0.049, \lambda=$ $0.00429, N=10000$, Gaussian, $\Gamma_{r m s}=0.4, \rho_{r m s}=$ 0.1 


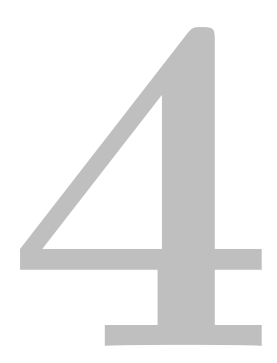

\section{Sound Generation due to Turbulence}

The sound generated by vorticity in an unbounded fluid is called aerodynamic sound $[15$,

16]. Pressure fluctuations occur in an unsteady flow in order to balance the fluctuations in momentum. Such fluctuations propagate outward from their source and, if an observer is present, subsequently recognised as sound [22]. Pressure variations at low Mach numbers are substantially uninfluenced by the effect of compressibility in the vicinity of localised flows and can be determined with the help of velocity fields by solving Poisson equations. Biot-Savart law relates the velocity field with vorticity field, which makes these pressure fluctuations in turn driven by a prescribed the vorticity field in an inviscid fluid.

\subsection{Aerodynamic Sound}

The aerodynamic noise arises because of two different phenomena. The first one is the impulsive noise, which is a result of moving surface or surfaces in nonuniform flow conditions. The displacement effect of an immersed body in motion and the unsteady aerodynamic loads on the body surface generate pressure fluctuations that are radiated as sound. This kind of noise is deterministic and relatively easy to extract from aerodynamic simulations because the required resolution in space and time to predict the acoustics is like the demands from the aerodynamic computations. If the surfaces move at speeds comparable to the speed of sound or there is an interaction between a rotor and a stator wake, these tonal noise components can be dominant. The other noise mechanism is the result of turbulence and therefore arises in nearly every engineering application. Turbulence is, by its very nature, stochastic, and therefore has a broad frequency spectrum. Turbulent energy is converted into acoustic energy most efficiently near sharp edges. As stated, turbulence noise exists almost every time; consequently, aerodynamic noise is usually a broadband noise sometimes augmented by narrow tonal components coming from impulsive noise sources [157].

The study of these flow generated noise probably began with Gutin's theory of propeller noise [158]. But it was Lighthill [15] who introduced his acoustic analogy to deal with the problem of jet noise that laid the foundation of modern aeroacoustics. His ideas were subsequently 
extended by Curle [17], Powell [159], and Ffowcs Williams and Hawkings [160] to include the effects of solid boundaries.

In this chapter, a brief mathematical description of equations used in airfoil-turbulence interaction noise is given. This is followed by employing the findings of chapter- 2 and 3 to build sources for aerodynamic noise for an airfoil which is then solved for far-field using the FW-H equations.

\subsubsection{Lighthill's Acoustic Analogy}

Lighthill proposed a method to calculate acoustic radiation from relatively small regions of turbulent flow surrounded by a quiescent fluid. It is no longer assumed that the flow in the source region is a linear perturbation of the reference state. The listener is surrounded by a quiescent reference fluid in which the small acoustic perturbations are accurately described by the homogeneous linear wave equation. The main idea behind Lighthill's analogy was to derive from the exact equations of mass conservation and momentum conservation a non-homogeneous wave equation that reduces to the homogeneous wave equation in a region surrounding the listener.

By taking the time derivative of the mass conservation law and subtracting the divergence of the momentum equation, an equation is obtained as

$$
\frac{\partial^{2} \rho}{\partial t^{2}}=\frac{\partial^{2}}{\partial x_{i} \partial x_{j}}\left(\mathcal{P}_{i j}+\rho v_{i} v_{j}\right)
$$

Subtracting $c_{0}^{2} \frac{\partial^{2} \rho}{\partial x_{i}^{2}}$ from the above equation leads to

$$
\frac{\partial^{2} \rho}{\partial t^{2}}-c_{0}^{2} \frac{\partial^{2} \rho}{\partial x_{i}^{2}}=\frac{\partial^{2}}{\partial x_{i} \partial x_{j}}\left(\mathcal{P}_{i j}+\rho v_{i} v_{j}-c_{0}^{2} \rho \delta_{i j}\right)=\frac{\partial^{2} T_{i j}}{\partial x_{i} \partial x_{j}} .
$$

The left-hand side of Eq. ( $\underline{4.2}$ now clearly represents the wave operator for a non-moving homogeneous medium with a constant speed of sound of $c_{0}$. The right-hand side represents a quadrupole source term. The quantity $T$ is called Lighthill's stress tensor. By introducing $p^{\prime}=p-p_{0}, \rho^{\prime}=\rho-\rho_{0}$ as deviations from respective constant reference values of pressure $p_{0}$, density 0 and speed of sound $c_{0}$, Eq. $\underline{(4.2)}$ can be written like:

$$
\frac{\partial^{2} \rho^{\prime}}{\partial t^{2}}-c_{0}^{2} \frac{\partial^{2} \rho^{\prime}}{\partial x_{i}^{2}}=\frac{\partial^{2}}{\partial x_{i} \partial x_{j}}\left(\rho v_{i} v_{j}+\delta_{i j}\left(p^{\prime}-c_{0}^{2} \rho^{\prime}\right)\right)=\frac{\partial^{2} T_{i j}}{\partial x_{i} \partial x_{j}} .
$$

For low Mach number flows with $M_{\infty} \ll 1$, the Lighthill tensor can be approximated as

$$
T_{i j}=\rho v_{i} v_{j}
$$

\subsubsection{Curle's equation: noise from stationary objects in low-speed flows}

The two major extensions of Lighthill's acoustics analogy are introduced by Curle [17] for solid walls at rest, and Ffowcs-Williams and Hawkings [160] for moving surfaces. The acoustic analogy can be generalised for flows in the presence of walls. Curle's approach includes the use of free-space Green's function $G_{0}[157,161]$. It is usually assumed that the ambient medium is in a mean state of rest at vast distances from the bodies and source region, and 


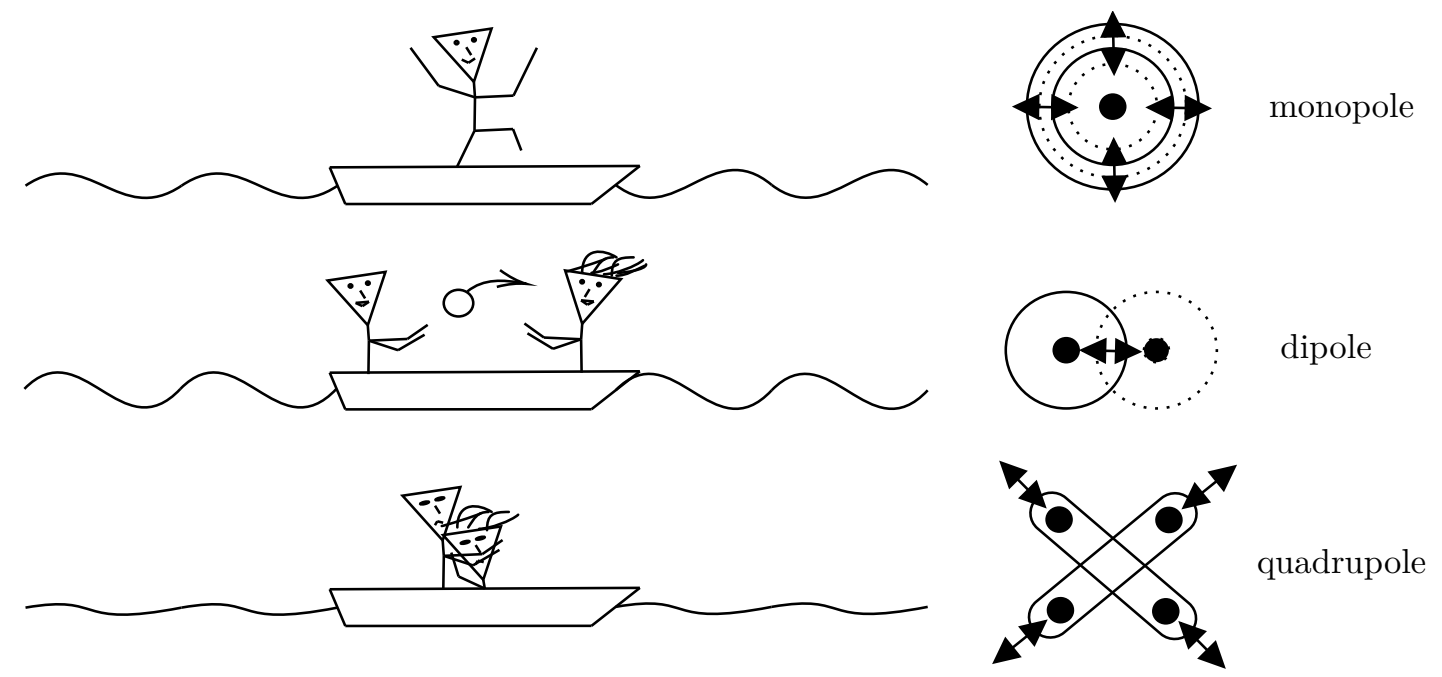

Figure 4.1: Monopole, dipole, and quadrupole generating waves on the surface of the water around the boat (inspired by Wagner, Hüttl, and Sagaut [157]). A person jumping up and down in the boat, producing an unsteady volume injection, thus generating a monopole wave field (top); two persons on the boat playing with a ball, exerting a force on the ball each time which results in the generation of an oscillating force which in turn generates a dipole field (middle); two individuals fighting with each other is a reasonable model for quadrupole (bottom).

an integral representation of the acoustic pressure $p \approx c_{0}^{2}\left(\rho-\rho_{0}\right)$ analogous to the Kirchhoff integral formula is formed in terms of the free space Green's function ${ }_{\text {_. }}^{1}$ The most general representation of this kind is the Ffowcs Williams \& Hawkings equation and is applicable for a control surface in arbitrary motion. However, only the limiting case of the stationary surface is considered in this thesis, which is also known as Curle's formula $[17, \underline{22}, \underline{157}, 162]$ :

in time-domain

$$
\begin{aligned}
p^{\prime}(\mathbf{x}, t)= & \underbrace{\frac{\partial^{2}}{\partial x_{i} \partial x_{j}} \int_{V}\left[T_{i j}\right]_{\tau=\tau^{*}} \frac{d^{3} \mathbf{y}}{|\mathbf{x}-\mathbf{y}|}}_{\begin{array}{c}
\text { free-field noise } \\
\text { quadrupole source }
\end{array}} \\
& \underbrace{-\frac{\partial}{\partial x_{i}} \oint_{S}\left[\rho v_{i} v_{j}+\mathcal{P}_{i j}\right]_{\tau=\tau^{*}} \frac{n_{j} d S(\mathbf{y})}{4 \pi|\mathbf{x}-\mathbf{y}|}}_{\begin{array}{c}
\text { loading noise } \\
\text { dipole source }
\end{array}} \\
& +\underbrace{\frac{\partial}{\partial t} \oint_{S}\left[\rho v_{j}\right]_{\tau=\tau^{*}} \frac{n_{j} d S(\mathbf{y})}{4 \pi|\mathbf{x}-\mathbf{y}|}}_{\begin{array}{c}
\text { thickness noise } \\
\text { monopole source }
\end{array}}
\end{aligned}
$$

where $\mathcal{P}_{i j}=\left(p-p_{0}\right) \delta_{i j}-\sigma_{i j}$, the surface normal $n_{j}$ is directed into the fluid, retarded time $\tau^{*}=t-\left(|\mathbf{x}-\mathbf{y}| / c_{0}\right.$. The propagation takes place from a source placed at $\mathbf{y}$ to an observer at $\mathbf{x}$. Curle's equation describes the sound field generated by a body immersed in a flow field. According to the three terms, three distinct contributions to this sound field are seen. The first term represents nothing but the sound field produced in the free fluid volume, a quadrupole source, which we know from the solution of Lighthill's equation for the free field. The second term is obviously related to the local forces acting on the surface of the object. In fact, if the

\footnotetext{
${ }^{1}$ For a detailed derivation, please refer to the lecture notes in physics by Auregan et al. [162]
} 
body surface is rigid and non-moving, then $v_{j}=0$ and the integral represents almost the net aerodynamic force, acting on the fluid. There is however a subtle difference, mainly the terms are to be evaluated at the retarded time and weighted by $|\mathbf{x}-\mathbf{y}|^{-1}$, which varies along the body length. Nevertheless, this part of the sound field is called "loading noise", dipole source. Finally, the third term is related to the displacement of the object. Obviously, if the body is at rest then again $v_{j}=0$ and this third contribution of the sound field is zero; it is called "thickness noise", a monopole source. Fig. (․1) shows the analogy of the waves generated by a boat on the water surface to understand monopole, dipole, and quadrupole.

The above equation is the general form which can be modified to account for the operating conditions. The first term, the volume integral, is referred to as the quadrupole term. For low Mach number flows $M_{\infty} \ll 1$, the volume source terms of quadrupole order are negligible compared to the surface source terms that are of dipole order $\stackrel{2}{2}$. Next, consider the dipole term of Eq. (4.5), which is the surface integral with terms related to the surface loading. Since the integral is in written in spatial derivative, an interchange in the derivative is carried out using the chain rule which yields an alternative form of dipole acoustic field:

$$
p^{\prime}(\mathbf{x}, t)_{\text {dipole }}=\int_{S}\left[\frac{\partial\left(\mathcal{P}_{i j}+\rho v_{i} \mho_{j}\right)^{0}}{\partial \tau}+\frac{\left(\mathcal{P}_{i j}+\rho v_{i} v_{j}\right) e_{0}}{|\mathbf{x}-\mathbf{y}|}\right]_{\tau=\tau^{*}} \approx 0 \frac{\left(x_{i}-y_{i}\right) n_{j} d S(\mathbf{y})}{4 \pi|\mathbf{x}-\mathbf{y}|^{2} c_{0}}
$$

For an impermeable surface $\rho v_{i} v_{j}=0$, and the only relevant term is the comprehensive stress tensor $\mathcal{P}$. The second term in square brackets becomes essential when the observer is close to the source but becomes less and less critical when the observer moves away from the source region, as is often the case. In present work, and most other applications, an only far-field approximation of Eq. (4.6) for a rigid stationary surface, which is

$$
p^{\prime}(\mathbf{x}, t)_{\text {dipole }}=\frac{x_{i}}{4 \pi|\mathbf{x}|^{2} c_{0}} \int_{S}\left[\frac{\partial\left(\mathcal{P}_{i j} n_{j}\right)}{\partial \tau}\right]_{\tau=\tau^{*}} d S(\mathbf{y}) .
$$

The last integral corresponds to monopole sound field generated by the mass flux through the surface $S$. If the surface is rigid or impenetrable (and of course stationary) then this term is zero.

The above equation is written in time-domain. With the use of a Fourier transform,

$$
\begin{gathered}
\mathcal{F}\{f(t)\}=\widehat{f}(\omega)=\int_{-\infty}^{\infty} f(t) e^{-i \omega t} d t, \\
\mathcal{F}^{-1}\{\widehat{f}(\omega)\}=f(t)=\frac{1}{2 \pi} \int_{-\infty}^{\infty} f(\omega) e^{i \omega t} d t,
\end{gathered}
$$

Eq. (4.7) can be expressed in the frequency domain as

frequency-domain

$$
\left.\widehat{p^{\prime}}(\mathbf{x}, \omega)=\frac{i \omega}{4 \pi c_{0}} \int_{S} e^{-i \omega|\mathbf{x}-\mathbf{y}| / c_{0}}\left(\widehat{\mathcal{P}_{i j}} \widehat{n_{j} \cdot\left(x_{i}-y_{i}\right.}\right)\right) \frac{d S(\mathbf{y})}{|\mathbf{x}-\mathbf{y}|^{2}}
$$

\footnotetext{
${ }^{2}$ Please refer to Aeroacoustics of Low Mach Number Flows by Glegg and Devenport [163] for the detailed mathematical explanation
} 


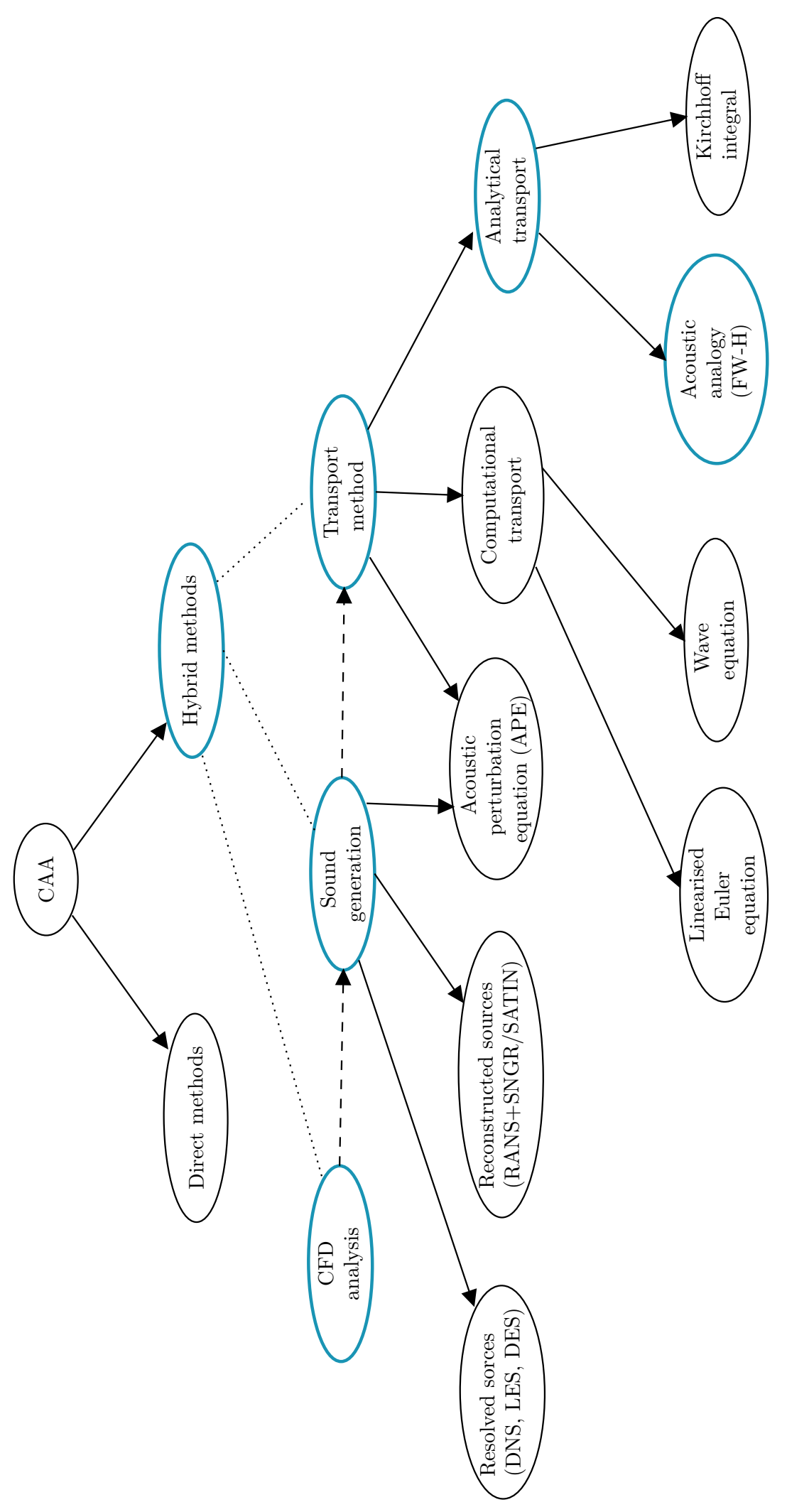

Figure 4.2: Noise prediction methods 
where $\widehat{p^{\prime}}$ and $\widehat{\mathcal{P}}$ are the Fourier transforms of $p^{\prime}$ and $\mathcal{P}$, respectively, and $\omega$ is the cyclic frequency. Fig. ( $\underline{4.2}$ ) shows different techniques which make up the maps of noise prediction methods, and also highlights the approach considered in this thesis.

\subsection{Airfoil-turbulence interaction / Leading-edge Noise}

A noise prediction methodology is characterised by its ability 1) to predict the response to large coherent disturbances accurately, and 2) to model statistically optimised artificial turbulence. Both of these characteristics are mainly studied independently under numerical methods, while these are mutually dependent on one another. The wrong prediction of the latter eliminates the possibility of the correct prediction of the former one, and prediction of noise radiation from a body in turbulent flow is a perfect example of it. A wide range of numerical work on airfoil-turbulence interaction noise is available, Lockard and Morris [38], Allampalli et al. [164], and Glegg and Devenport [165] studied the effects of geometry on unsteady aerofoil interaction noise. A thorough review of the literature on analytical, numerical, and experimental work on leading-edge is discussed in chapter-1.

In this chapter, modelling of the interaction of inflow fluctuating velocity signal with an airfoil is discussed. The preceding chapters have presented the implementation of the vortex method, a Lagrangian based approach, to solve for the flow characteristics around a body. The solver is written in python and is validated against the results of standard cases from xfoil. Following, the inflow turbulent is modelled using vortices which exhibit the randomness as well as behave in such a way that the desired statistics are achieved. The modelling of airfoil-turbulence interaction phenomena require the methodology to accurately calculate the unsteady aerodynamic forces over the surface of the body, which are then used to compute the radiated sound pressure in far-field. In the next section, steady background flow around an airfoil is calculated.

\subsubsection{Steady background flow}

Further research which is leading to the explanation of proposed technique to predict the acoustic radiation from an airfoil while interacting with an incoming turbulent flow is carried out on the NACA0012 airfoil. In previous chapter, it is shown that a set of discrete vortex particles arranged in a definite manner, controlled by their position, shape, strength, and size, can replace a realistic turbulent velocity field. The spatial distribution of these particles was so random that if they were convected in the same way around an airfoil, the induced velocity on the panels of the airfoil due to the particles would be nonphysical, which is redundant. In order to deal with this, it is imperative to pass these vortices around an airfoil in streamline $\mathrm{C}_{-}^{3}$ pattern.

This requirement states the main objective of this section, to get the pathlines for the vortex particles for their convection downstream in the streamwise direction. Steady flow simulation of NACA0012 airfoil is performed using the potential code developed in Chapter 2. The upper and lower surface of this $12 \%$ thick and $1 \mathrm{~m}$ extended chord length profile is discretised in 100 panels each, which yield 200 panels in total, as shown in Fig. (4.3a). The airfoil is placed in the

\footnotetext{
${ }^{3} \mathrm{~A}$ streamline is defined as a line which is everywhere parallel to the local velocity vector [85]
} 


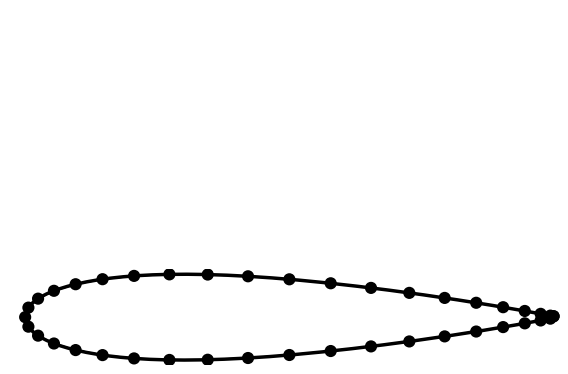

(a) discretised NACA0012 airfoil

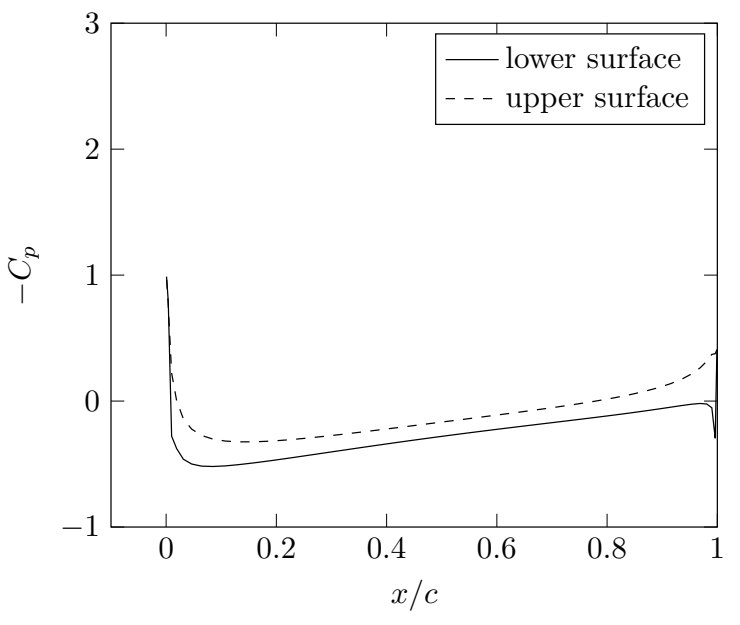

(b) $\mathrm{Cp}$ at $\alpha=0^{\circ}$

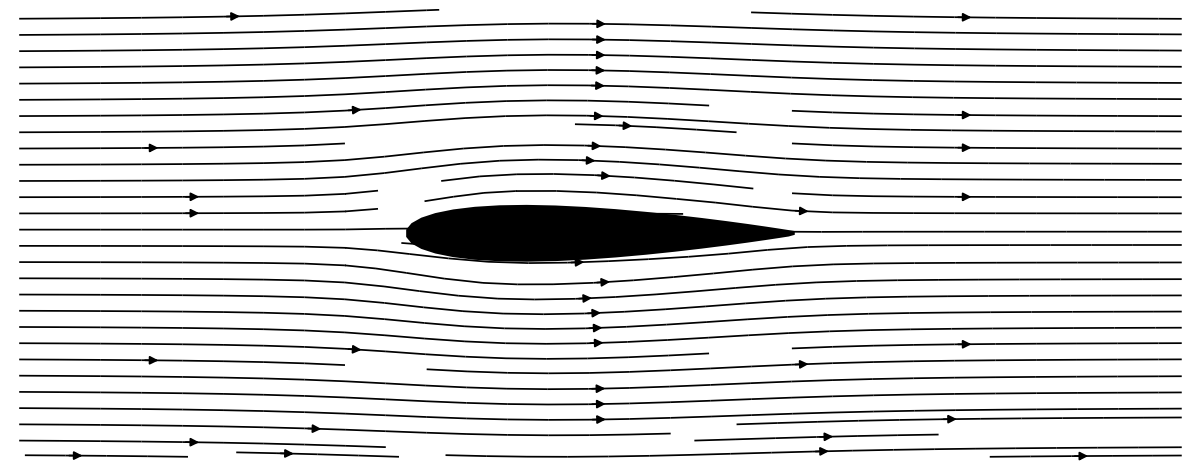

(c) streamlines around NACA0012 airfoil due to $U=1 \mathrm{~m} / \mathrm{s}$ and $\alpha=0^{\circ}$

Figure 4.3: Steady background flow around NACA0012 airfoil using the panel method

$x y$-plane with its chord line parallel to the $x$-axis, and leading-edge coinciding with the origin. The airfoil is immersed in a free-stream $(U, \alpha)$ where $U=1 \mathrm{~m} / \mathrm{s}$ and $\alpha=0^{\circ}$ is the velocity magnitude and angle of attack, respectively. Each discretised panel is characterised by its start and endpoints, centre point, length, orientation, source strength and vortex-sheet strength. The system of equations is constructed using the flow tangency condition and enforcing the Kutta condition at the trailing edge which results in $(200+1)$ equations for $(200+1)$ unknowns. For complete implementation, please refer to chapter-2.

Fig. (4.3) shows the results of the steady-state simulation. The negative pressure coefficient is plotted along the positive $y$-axis, and the pressure coefficient is observed to stagnate at the leading-edge $(C p=1)$ in Fig. (4.3b). The equation for the streamline can be written as an Ordinary Differential Equation for the streamline shape $\mathrm{y}(\mathrm{x})$ :

$$
\frac{d y}{d x}=\frac{v}{u}
$$

Again, $u(x, y)$ and $v(x, y)$ must be given to allow integration, and $x_{p}, y_{p}{ }_{-}^{4}$ must be given to set the integration constants. In numerical integration, $x_{p}, y_{p}$ serve as the initial values. This

\footnotetext{
${ }^{4} x_{p}, y_{p}$ is abscissa and ordinate of the point where the velocity vector is calculated
} 
Table 4.1: Parameters for steady-state analysis of flow around NACA0012 airfoil

\begin{tabular}{ll}
\hline Parameters & Values \\
\hline Airfoil & NACA0012 \\
Chord length & $1 \mathrm{~m}$ \\
Number of panels & 200 \\
Panel singularity & Source and vortex \\
$U$ & $1 \mathrm{~m} / \mathrm{s}$ \\
$\alpha$ & $0^{\circ}$ \\
\hline
\end{tabular}

appears in the flow for the airfoil in Fig. $\left(\underline{4.3 \mathrm{c})}\right.$. As a result of $\alpha=0^{\circ}$, there is a symmetric distribution of streamlines about the $x$-axis. Accordingly, the streamlines to the flow around the leading-edge of the airfoil and meet smoothly at the trailing easily.

\subsubsection{Acoustic radiation due to a single vortex}

The determination of streamlines in the previous section overcomes a huge challenge for the convection of the vortices. Where previously, randomly scattered vortices convecting in the streamwise direction could induce nonphysical velocity over the airfoil surface, it is now easier to inject the vortices at the beginning of a streamline, making it follow the trajectory in stream-wise direction. Mean flow and unsteady computations are conducted independently. The mean flow calculation, performed earlier, is integrated to determine the streamline. These streamlines define the convection path for the injected vortices. The unsteady calculation is then conducted repeatedly to obtain the linear perturbations to this solution produced by discrete vortices originated at different heights from the stagnation streamline and allowed to convect across the domain with an airfoil placed at origin as described in sec. (4.2.1).

\subsubsection{Implementation}

Fig. (4.4) shows the set-up where an airfoil, discretised into straight panels of finite length, with its leading-edge facing opposite to the stream-wise direction, is placed with its chord line parallel to the horizontal axis. The introduction/injection of vortices into the domain requires an imaginary line of finite length which behaves as pseudo inlet in our Lagrangian system. The length of this imaginary window is kept five chord lengths upstream from the leading-edge of the airfoil, and five chord length downstream from the trailing edge of the airfoil. The dotted line in the far-field upstream represents the injection line. The injection line is a finite length imaginary straight line aligned perpendicular to the $x$-axis from which the vortices enter into the domain. The length of this line is equal to twice the separation between the centre of the weakest and smallest vortex to the farthest point at which the corresponding fluctuation is induced as discussed in sec. (3.6.5).

A vortex particle mollified using Mexican-hat waveform of strength $\Gamma$ and radius $R$ is released well upstream to the airfoil, $x_{o}$, at a particular vertical distance, $y_{o}-$ which is random, from the stagnation streamline. The particle follows the precomputed trajectory well downstream the airfoil, which is calculated using the mathematical description of streamlines given in the last section. For the present analysis, $x_{0}$ equals -3.0 . and $y_{0}$ takes a random value in order to facilitate a uniform distribution to the ordinate values. As soon as a vortex particle 


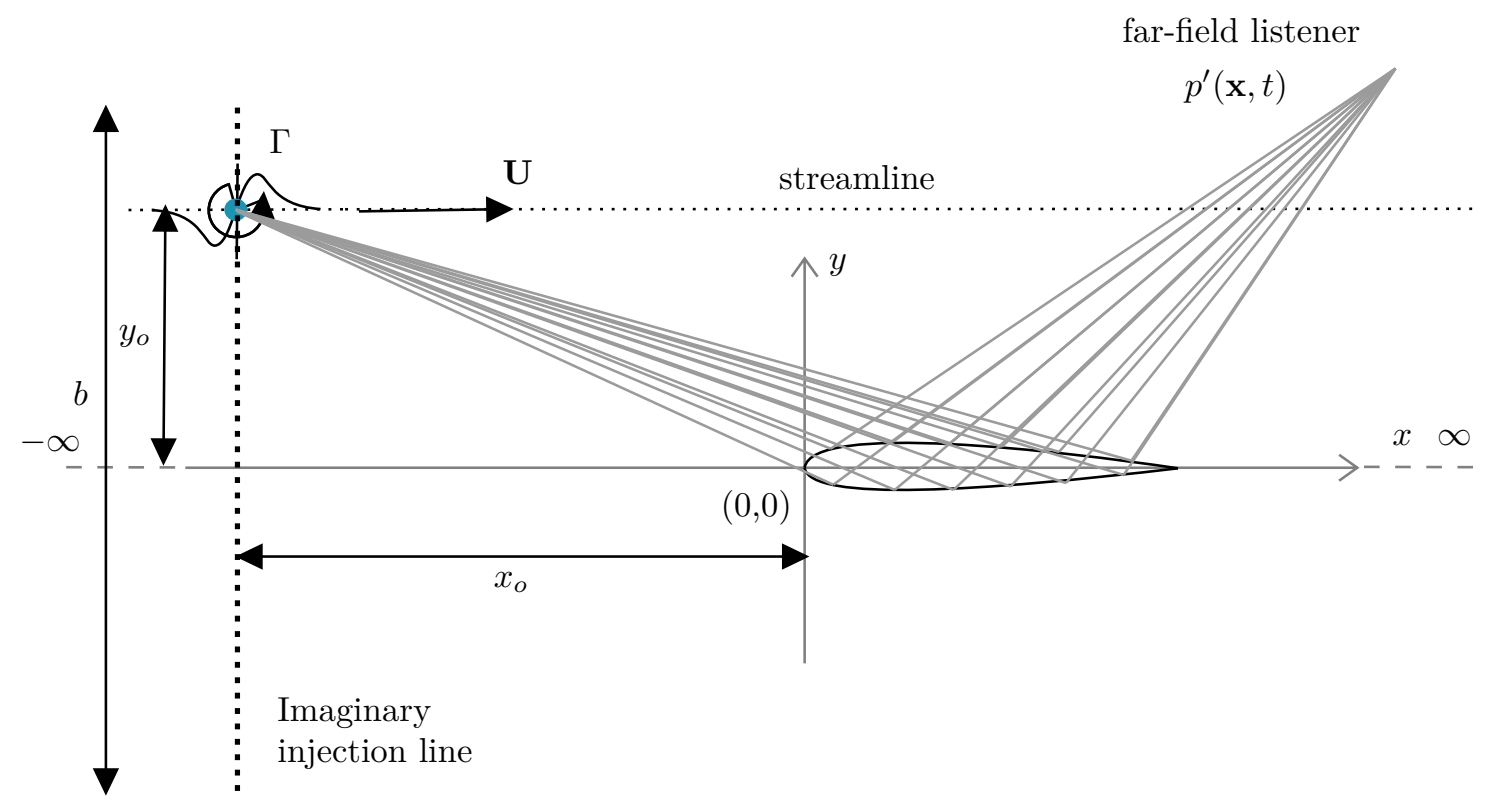

Figure 4.4: Acoustic field due to the interaction of a single vortex injected from the injection line in the streamwise direction

begins moving from its point of origin, $\left(x_{o}, y_{o}\right)$, and convects along the streamline downstream, the corresponding induced velocity on each panel of the airfoil starts getting recorded. Since the free-stream velocity is $1 \mathrm{~m} / \mathrm{s}$, it takes the particle 11 seconds to move from $x=-5.0$ to $x=\left(c_{a}+5.0\right)$, making the total time of simulation to be 11 seconds. Acknowledging the strength magnitude of a single vortex, it is expected that its induced fluctuating disturbances will result in low-frequency sound radiation. The time-step of for the analysis of 0.001 which gives a frequency sampling of $500 \mathrm{~Hz}$. During this entire calculation of induced velocity over the surface of the airfoil, the fluctuating velocity is transformed to fluctuating pressure with the help of unsteady Bernoulli's equation (sec. (2.6.4)) and used as input into Curle's frequency domain formula for dipole radiation (Eq. (4.10)). The listener/observer is stationed at $(x=2.0, y=2.0)$. The main purpose of using Curle's frequency domain formula instead of the time-domain formula is to eliminate the complexity involved in calculating the effect of retarded time. Due to a large number of panels on the airfoil surface, using the Curle's time-domain formula, it would be necessary to first find the correct $d \tau$ value for each panel to account for the retarded time, which can lead to an increase in the computational cost. However, in frequency-domain formula, $\exp (-i k r)$ term takes care of retarded time efficiently. A total of three cases, listed in Table. (4.2), are derived to study the the interaction of a single vortex particle with an airfoil at rest. The derivation of cases is based on difference in size, injection point, and strength.

The first case, case-1, has four subcases in which a single vortex is released in each case from an identical vertical distance above the stagnation streamline. The strength $\Gamma$ for all the vortex particles are equal, however, the variation is brought to the radius $R$ of the vortices. The sound pressure levels from all the four subcases are fundamentally different and do not scale with a factor, as shown in Fig. (4.5a). This observation supports and explained by the fact that the induced velocity by the vortex does not depend linearly on the vortex radius. Similarly,

\footnotetext{
${ }^{5}$ injection point
} 
Table 4.2: Vortex parameters for a single particle

\begin{tabular}{llll}
\hline Case & $y_{0} \underline{5}$ & $\Gamma$ & $R$ \\
\hline & 0.01 & 1.0 & 0.15 \\
Case-1 & 0.01 & 1.0 & 0.20 \\
& 0.01 & 1.0 & 0.25 \\
& 0.01 & 1.0 & 0.30 \\
& & & \\
Case-2 & -0.08 & 0.50 & 0.55 \\
& -0.018 & 0.50 & 0.46 \\
& -0.015 & 0.50 & 0.96 \\
& -0.037 & 0.50 & 0.32 \\
Case-3 & 0.01 & 0.25 & 0.20 \\
& 0.01 & 0.50 & 0.20 \\
& 0.01 & 0.75 & 0.20 \\
& 0.01 & 1.0 & 0.20 \\
Case-4 & 0 & -0.3826 & 0.20 \\
& 0 & 0.8477 & 0.20 \\
& & & \\
Case-5 & 0 & 0.50 & 0.31 \\
& 0 & 0.50 & 0.44 \\
\hline
\end{tabular}

case-2 with identical vortex strength but different injection point and radius show the same behaviour. It is evident in Fig. (4.5b) that the SPL do not scale or/and shift vertically and horizontally with a factor of finite number for vortices with different sizes irrespective of their point of origin in the upstream far-field. However, the vortices of the same size originated from the same point but with different strength, case-3, show a scaling/shifting pattern in the SPL spectra. Fig. (4.5c) shows four identical spectra with different intercepts on $y$-axis. This behaviour can be explained by the linear dependence of vortex strength variable on the induced velocity by a vortex, also discussed in sec. (3.6.3).

Fig. (4.5d) shows the results of a study conducted to understand the effect of presence time of a vortex in a domain on sound pressure level spectra. In previous study, a vortex started from $x_{o}=-5.0$ and convected until $x_{o}=c_{a}+5.0$, whereas in present study, three more cases of different presence times are considered. The least being $x_{o}=-1.0$ to $x_{o}=c_{a}+1.0$, and highest being $x_{o}=-10.0$ to $x_{o}=c_{a}+10.0$. It is clearly visible that the plots are parallel to each other but not coinciding with each other.

The vortices which travel close to the airfoil surface, i.e. with trajectories close to the surface, show a fascinating behaviour. The solution seems to oscillate wildly if the distance between the vortex and the airfoil surface becomes less than the scale of panel length. Two more cases with vortices trajectories coinciding the stagnation line are simulated in the same domain. Glegg and Devenport [165, 49] suggested in their work that near-surface vortex trajectories can also cause problems in their interaction with the trailing edge. This interaction should produce a singularity in the response that is exactly cancelled by the Kutta condition. In the 


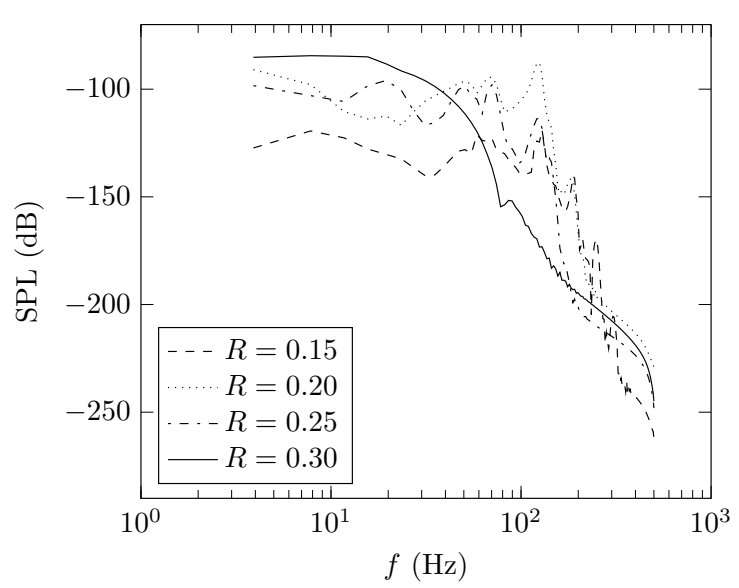

(a) $y_{o} 0.01, \Gamma=1.0, R=$ variable

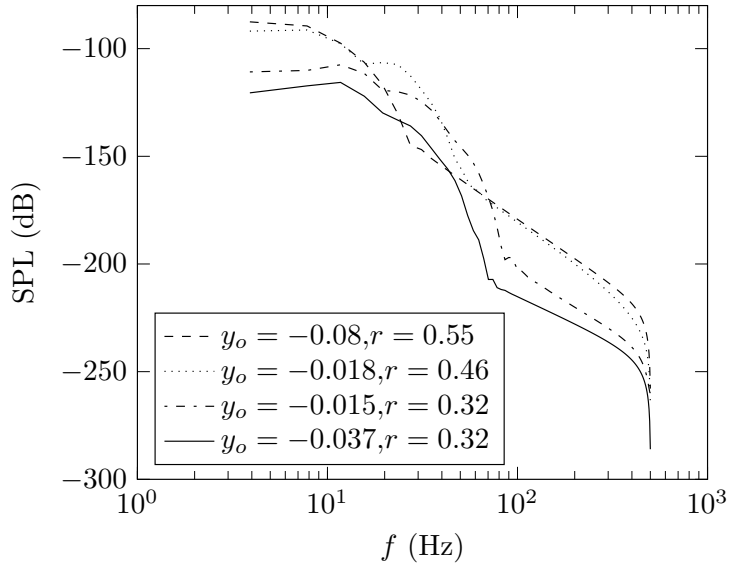

(b) $y_{o}=$ variable, $\Gamma=0.50, R=$ variable

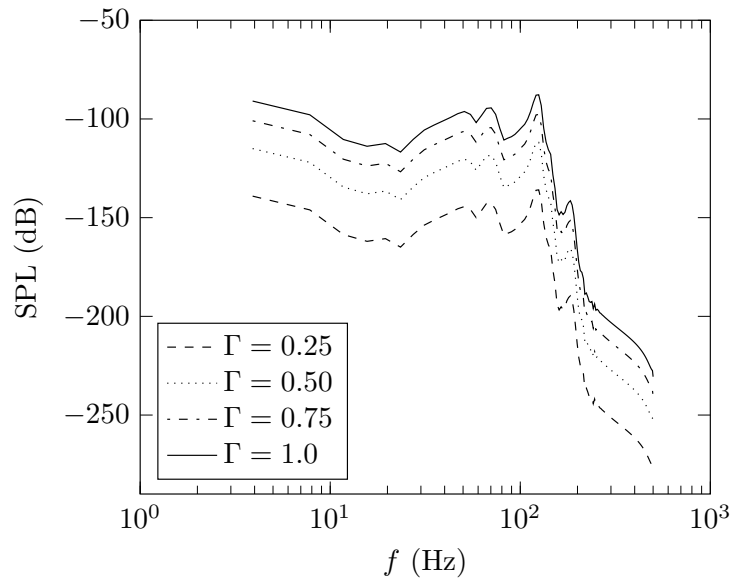

(c) $y_{0} 0.01, \Gamma=$ variable, $R=0.20$

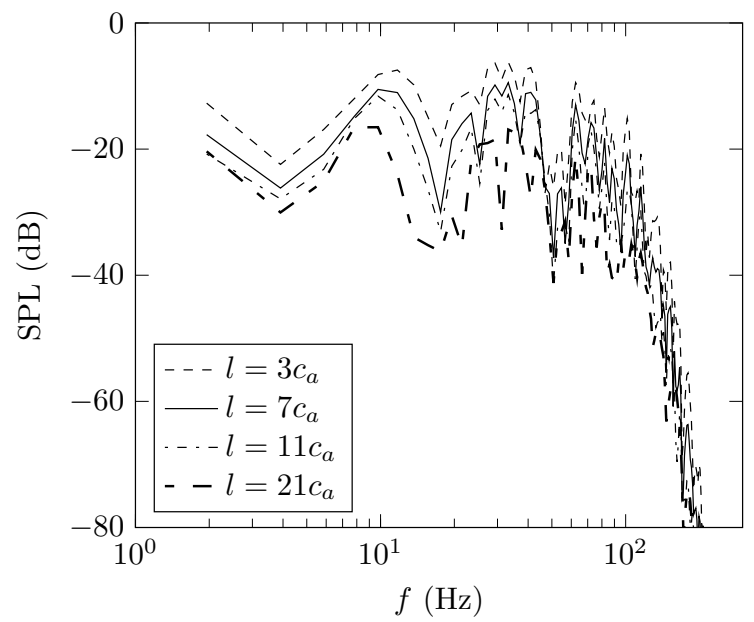

(d) $y_{o} 0.01, \Gamma=1.0, R=0.20$

Figure 4.5: single particle acoustic signature 


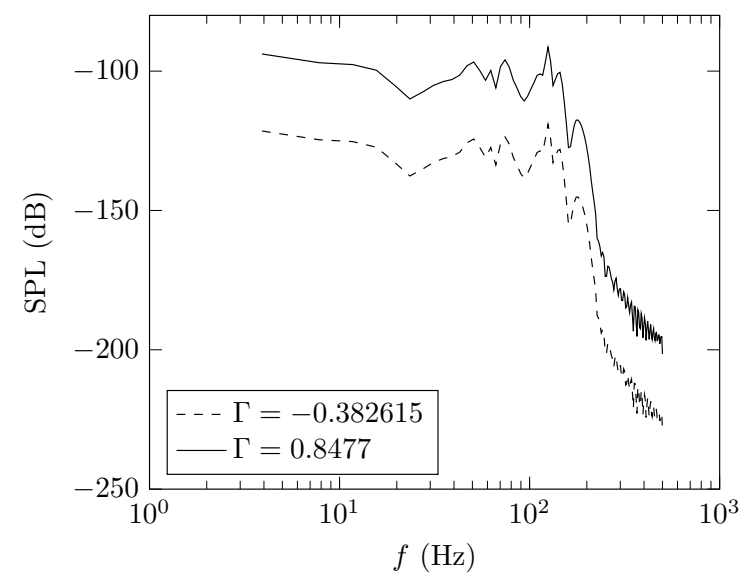

(a) $y_{o} 0, \Gamma=$ variable, $R=0.2$

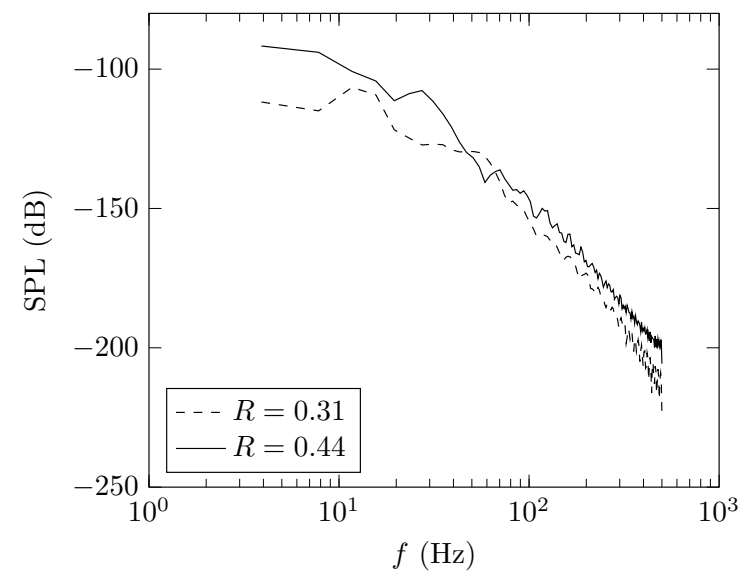

(b) $y_{o} 0, \Gamma=0.50, R=$ variable

Figure 4.6: stagnation streamline acoustic signature

panel method, however, the cancellation is usually not perfect. The residual spike can result in some scalloping of the computed airfoil response function and predicted sound spectrum. This effect can be minimised by using panels of a constant length in the trailing edge region, by using a low-order representation of the wake, hence the present use of discrete wake vortices rather than panels. Fig. ( $\underline{4.6}$ ) shows the highly fluctuating spectra for two cases mentioned as case-4, identical size but different strength, and case-5, different size but identical strength, in Table. (4.2).

Scaling in pressure The variation in vortex strength induces a corresponding shift in sound pressure levels that can be seen evidently in Fig. (4.5c) where SPLs due to vortices of identical size and different strength exhibit similar shape spectra parallel to each other with different intercepts on $y$-axis. However, a shift can be observed in the vertical direction. This vertical shift is studied with a larger number of vortices, 31 to be exact, to clearly understand the behaviour of intercept shift. The injection line is situated at $x_{o}=-3.0$, and the vortices are injected from $y_{o}=0.01$ above the stagnation line. The strength of 31 particles of identical size, $R=0.4$, are characterised by $\Gamma_{n} \in\{0.1,0.2, \ldots, 3.1\}$. The particles are convected through the streamline associated with the originating point 0.01 with a free stream velocity of $5 \mathrm{~m} / \mathrm{s}$. The induced velocity-pressure values are post-processed using Curle's formulation, which delivers the SPL data for 31 vortices, shown in Fig. (4.6a). The increment in $\Gamma$ values is reflected in corresponding sound spectra as a positive vertical shift of a principal/reference spectrum, which is due to the vortex of $\Gamma=0.1$. The gap between these parallel curves is seen to reduce in an unknown pattern when moving from $\Gamma_{1}$ to $\Gamma_{31}$, which is visualised in Fig. (4.6b). A logarithmic pattern seems to govern the gap between these curves, as shown in the figure. Using the LM algorithm to solve the least-square curve fitting method, a logarithmic function of form $f(x)=a \ln (x)+b$ is fitted to Fig. (4.6b) which yielded

$$
\mathrm{SPL}=\mathrm{SPL}_{\mathrm{ref}}+\left\{25.821 \ln \left|\Gamma-\Gamma_{\mathrm{ref}}\right|+81.035\right\}
$$




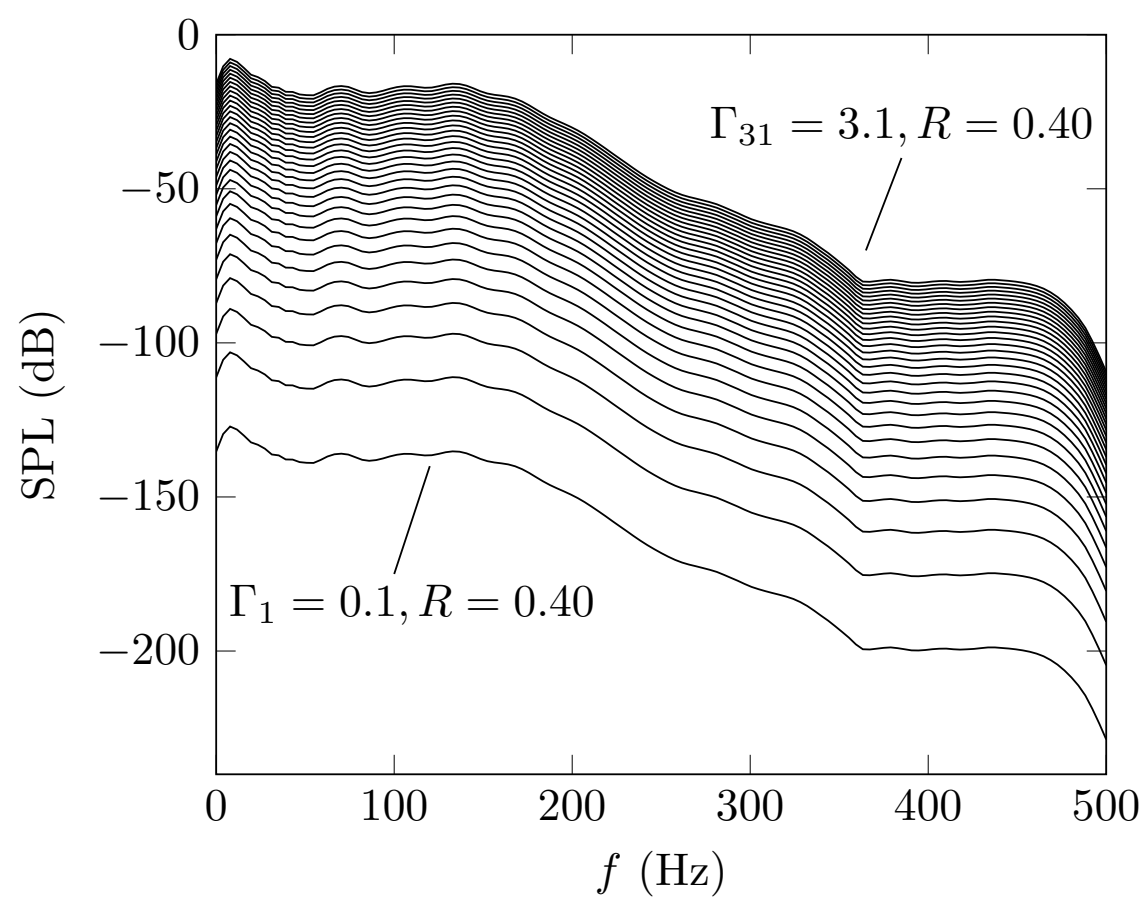

(a) $y_{0} 0.01, \Gamma=0.1--3.1, R=0.40$

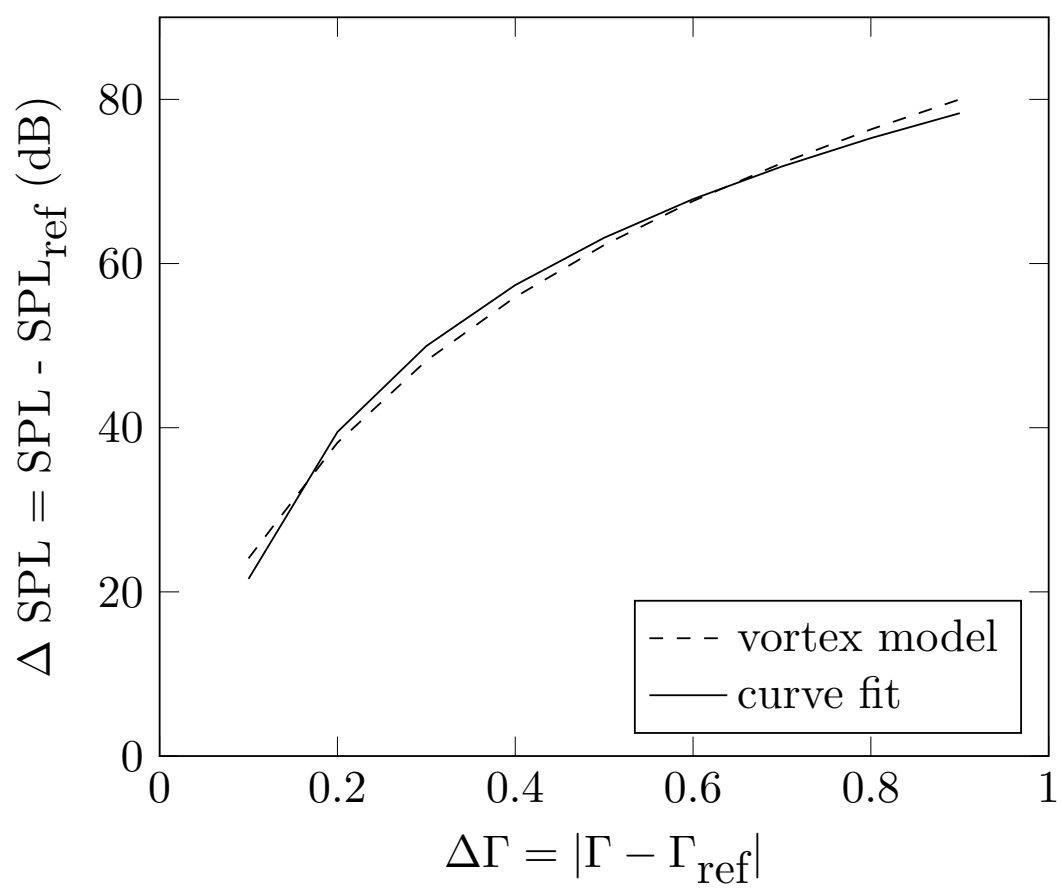

(b) SPL $-\Gamma$ curve

Figure 4.7: Pressure $\Gamma$ relation

Above equation enables a quick determination of SPL values at a point in the space due to the interaction of the identical size and varying strength vortices with an airfoil. This equation is important because it eliminates:

i) the requirement of calculating the unsteady loading due to every vortex (of course same size with variations in strength), and

ii) the post-processing of aerodynamic pressure to predict SPL. 
Hence, the sound radiation by an airfoil interacting with a stream of same size vortices no longer require computing the effect of each vortex at every single time step. In the subsequent sections, Eq. (4.12) is utilised develop the vortex-sound database, which has the potential to dramatically curb the computational cost involved in the prediction of airfoil-turbulence interaction noise.

Inference The previous chapter elaborated on the ability to create a frozen turbulent velocity field with the help of 10000 non-interacting vortices. A set of discrete vortices can be used to generate any required turbulent flow field by modifying their directional strength and size. But when it comes to modifying the strength and radius of 10000 Vortices, the main challenge then remains is to reduce the computational cost.

Suppose an attempt is being made to predict the acoustic radiation from an airfoil while subjected to flow fields of different turbulence intensities, $T u$ and length scales $\lambda$. It is possible to do so for a "single" Tu and $\lambda$ by merely allowing a set of vortices with their spatial arrangements, strength, and radius regulated by appropriate PDFs to convect around the airfoil. Hence, a change in $T u$ and $\lambda$ values demand a new set of vortices with different attributes. This leads to a monumental increment of calculation time if the airfoil needs to be analysed for more than one value of Tu and $\lambda$.

In the framework of present work, a database of $\mathrm{p}$ [recomputed farfield acoustic pressure due to the interaction of all possible vortices - based on all the possible combinations of $p(x)$, $p(y), p(\Gamma)$, and $p(R)$, with a particular airfoil is suggested. This allows to pick the sound pressure levels due to the interaction of a particular arrangement of vortices corresponding to a particular turbulence measuring parameters without simulating the entire case in real time.

\subsubsection{Acoustic radiation due to a single injection point}

The idea presented above is demonstrated here in this section for the vortices injecting out of a single point. The injection line is brought close to the airfoil at $x_{o}=-3.0$. The selection of this coordinate is based on the results of the research conducted in sec. (4.2.2.1). There is not much difference in the sound-induced by the vortex which started three chord lengths upstream of the airfoil and the vortex which started more or less than three chord lengths. While prolonging the length of the vortex window results in the drop of SPL values, on the other hand, it is essential to remember the fact that a two-dimensional study under predicts the SPL values $[166,167]$. The injection point located $0.01 \mathrm{~m}$ above the horizontal axis/stagnation streamline releases 500 Mexican-hat waveform vortices at an interval governed by the uniform distribution. This means that a snapshot of the domain taken after the introduction of all the vortices into it shows all the vortices uniformly distributed over the line of convection. This is to be noted that this line of convection is the trajectory followed by all the vortex particles injected from a same point. The strength and the radius of the particles are randomly distributed according to the normal and Rayleigh distribution, respectively. The Rayleigh distribution for the radius is restricted to allocate radius values in the interval $\{0.1,0.2,0.3,0.4,0.5\}^{6}$. For strength, the normal distribution can take any value since Eq. (․12) facilitates the estimation of sound

\footnotetext{
${ }^{6}$ The reason for restricting the values to the specified interval is discussed in sec. (4.2.3.1)
} 
pressure levels from the vortices of the same size and different strength by relating the radiated pressure with a precomputed reference pressure.

\subsubsection{Vortex-sound database}

The database stores the precomputed dipole sound radiating from the surface of a fixed airfoil interacting with all the possible vortices derived from the sample space of strength and radius. The following challenges for creating this database along with their solutions are listed below:

Strength sample space The strength of vortices in the computational domain is determined by the normal distribution, which is an infinite number line distribution. It is an extraordinary notion to consolidate countless strength cases in the database, but it is possible to estimate the sound-induced by a vortex of any strength with the help of Eq. (4.12) which relates the acoustic radiation from a vortex of any strength with the acoustic radiation from a vortex of a reference strength (e.g. $\Gamma_{r e f}=0.1$ ). This possibility is due to the linear relationship between velocity and strength gamma induced by the vortex.

Radius sample space The radius is controlled by Rayleigh distribution which is a semiinfinite number line distribution. Because of the nonlinear relationship between the radius and the vortex-induced velocity, variations in radius do not get reflected as scaling and shifts in spectral densities. The sample space of radius is, thus, limited to $\{0.1,0.2,0.3,0.4,0.5\}$ due to these two main reasons.

Implementation The parameters controlling the dynamics of the present analysis are listed in Table. ( $\underline{4.3)}$. The first diagram (top) in Fig. ( $\underline{4.8)}$ has an open slot on the injection line which symbolises the injection point. This point is located $0.01 \mathrm{~m}$ above the horizontal axis and is the source of origin of 500 vortices. A vortex of each size $(R \in 0.1,0.2,0.3,0.4,0.5)$ carrying a strength $\Gamma_{r e f}=0.1$ is convected over the streamline originating from $y_{o}=0.01$. The convection occurs at a time step $\Delta t=0.001$ with a freestream velocity $U=5 \mathrm{~m} / \mathrm{s}$ to give a total of 4200 iterations. The time-history of the fluctuating induced velocity on the airfoil surface is recorded and is transformed to fluctuating pressure with the help of unsteady Bernoulli's equation (sec. 2.6.4) and used as input into Curle's frequency domain formula for dipole radiation (Eq. $(\underline{4.10})$ ). The listener/observer is positioned at $(x=2.0, y=2.0)$. The sound radiation due to all the vortices is stored in a matrix form, as shown in Fig. (4.9).

After the database is ready, it is time to release 500 Vortices uniformly, "virtually". At $t=0$, the injection point randomly selects a value for strength and radius for the vortex from the database matrix, as shown in Fig. (4.9) and stores the SPL spectrum into a temporary variable. Since the injection is governed by a uniform PDF, it is not relevant to consider the injection time step, i.e. the time between the release of two vortices. Following, the radius and strength for the next vortex are chosen from the matrix. But the difference for this release is the time delay, hence the time difference between the previous vortex release and this vortex is considered by adding an extra term, $-\exp \left(j k\left(r+\tau_{v}\right)\right)$ to the $p^{\prime}(\mathbf{x}, t)$ before taking its spectral density. The corrected SPL is then added to the temporary variable which was already storing the noise spectrum from the previous vortex. This process continues similarly for the remaining 

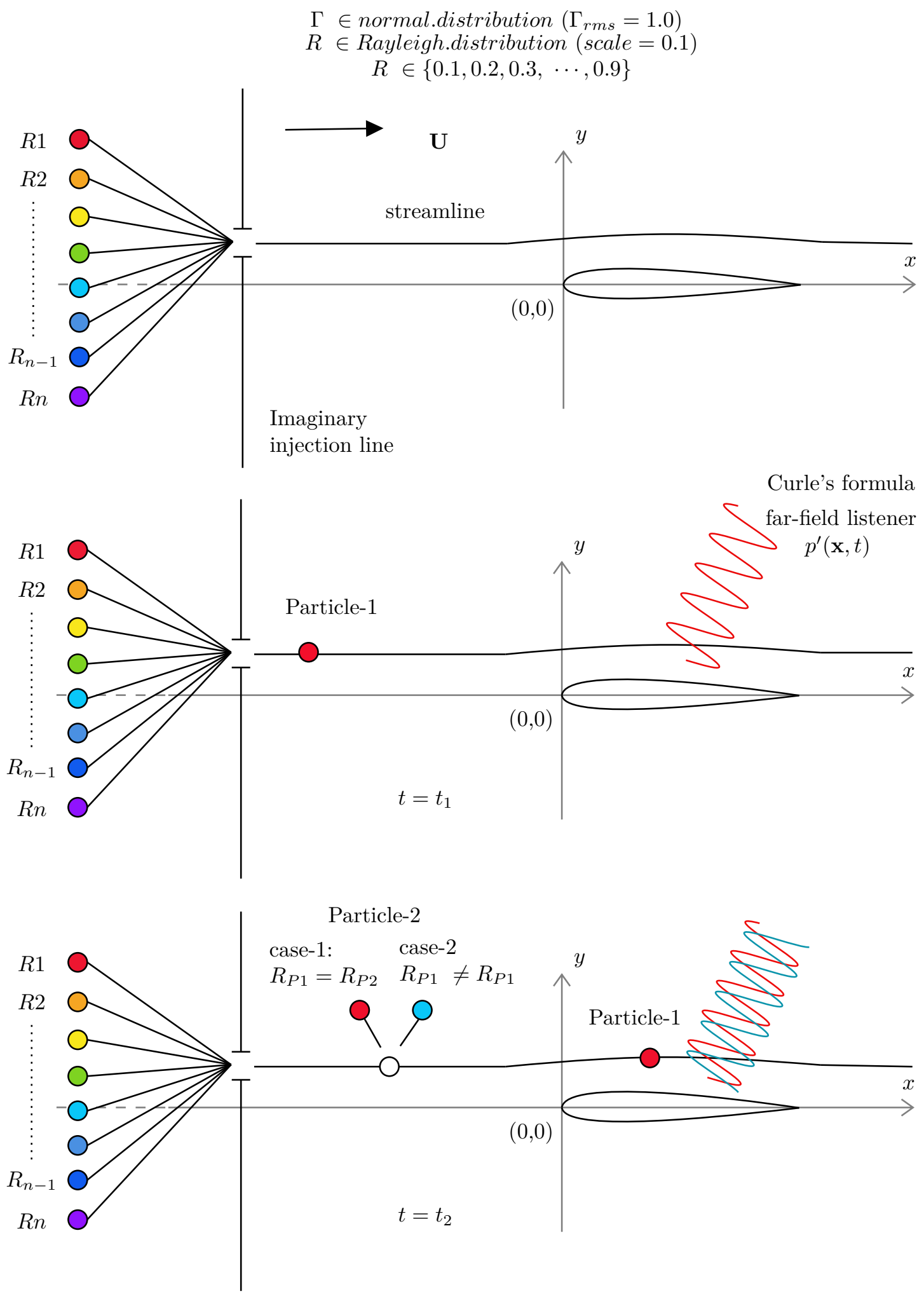

Figure 4.8: Convection of particles from a single injection point into the domain 


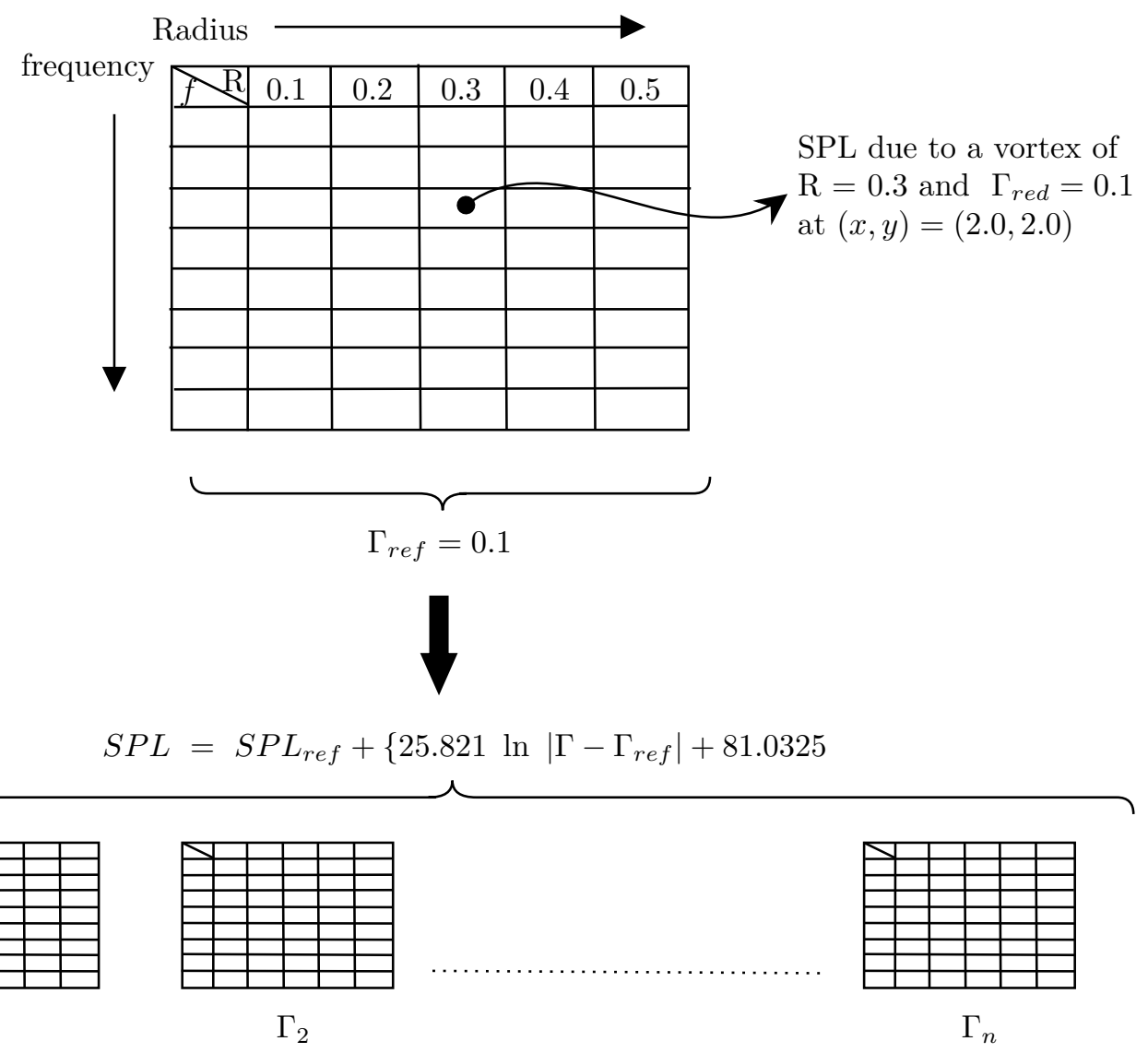

Figure 4.9: Database matrix of sound pressure levels for a single injection point

498 vortices and continues to be stored in the temporary variable. Subsequently, all SPL spectra are superimposed together according to the law of logarithmic addition, allowing the

Table 4.3: Parameters involved in the interaction of 500 vortices, injecting out of the same point, with NACA0012 airfoil

\begin{tabular}{ll}
\hline Parameters & Values \\
\hline$x_{o}$ & -3.0 \\
$y_{o}$ & 0.01 \\
Number of injection points & 1 \\
$\Gamma_{\text {ref }}$ & 1.0 \\
$\sigma_{R}$ & 0.1 \\
Vortex production rate & 110 \\
Number of vortex particles & 500 \\
Vortex type & Mexican-hat waveform \\
Airfoil & NACA0012 \\
Number of panels & 200 \\
Panel singularity & Source and vortex \\
$U$ & $5 \mathrm{~m} / \mathrm{s}$ \\
$\alpha$ & $0^{\circ}$ \\
Time step, $\Delta$ & 0.001 \\
Number of time steps & 4200 \\
Frequency sampling, $F_{s}$ & $1000 \mathrm{~Hz}$ \\
\hline
\end{tabular}




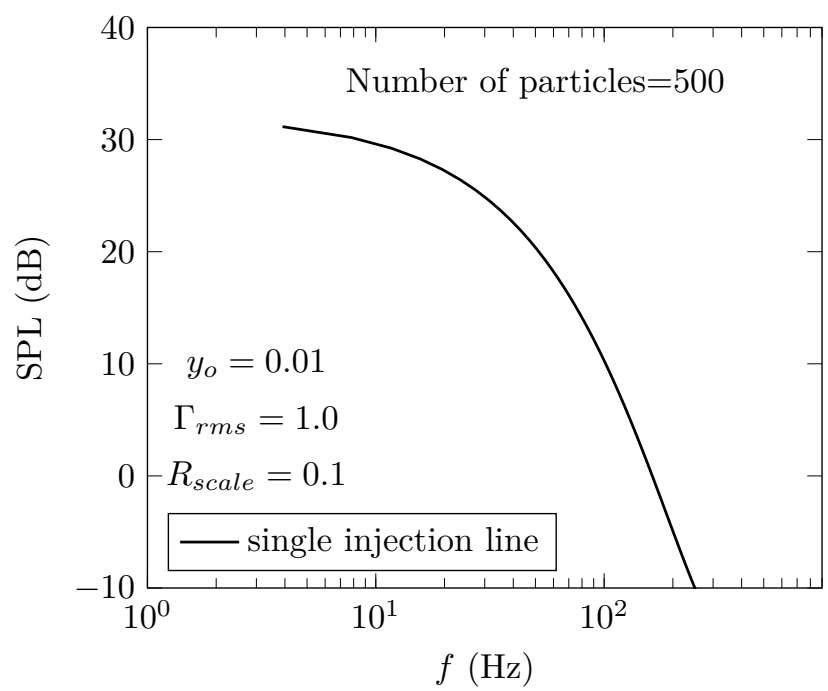

Figure 4.10: single streamline injection: 500 particles with $\Delta t=0.001 \mathrm{~s}$

estimation of dipole noise arising due to the unsteady loading developed over an airfoil due to the convection of 500 vortices. The SPL spectra are shown in Fig. (4.10).

\subsubsection{Reconstruction of the velocity field}

The approach to reconstruct a turbulent velocity field, as discussed in sec. (3.6) is utilised here to generate a statistically optimised fluctuating velocity field due vortices arranged in UUNR configuration convecting in straight lines resembling streamlines without any body of influence. In Fig. (4.11b)-(4.11e) velocity signals and spectra at the centre of the computational domain are shown. The vortices are arranged in UUNR configuration which means their spatial variables, $(x, y)$, are governed by the uniform distribution, strength, $\Gamma$, by the normal distribution, and radius by Rayleigh distribution_. The abscissa of the vortices spatial parameters are governed by the conventional uniform distribution. However, the ordinate is limited to 20 values which signify the 20 injection points on the imaginary injection line. Fig. (4.11a) shows the snapshot where 10000 Mexican-hat waveform vortex particles are distributed in the domain. 10000 particles are equivalent to the vortex production rate of 110 , which is highly stable in producing the identical spectra repeatedly. The resolution of the

\footnotetext{
${ }^{7}$ Interested readers may refer to section (3.6) for the reasoning behind choosing the specific distributions for the vortex control parameters
}

Table 4.4: Vortex parameters controlling the turbulence statistics for streamline convection

\begin{tabular}{|c|c|c|c|}
\hline Definition & $\begin{array}{l}\text { Stochastic } \\
\text { variable }\end{array}$ & Distribution & Range \\
\hline Spatial distribution & $p(x), p(y)$ & Uniform & $\begin{array}{c}x_{\min }=-l / 2 \\
x_{\max }=l / 2 \\
y=\{-0.1,-0.09, \ldots, 0.09\}\end{array}$ \\
\hline Strength distribution & $p(\Gamma)$ & Normal & $\sigma=1.0$ \\
\hline Radius distribution & $p(R)$ & Rayleigh & $\sigma=0.1$ \\
\hline
\end{tabular}




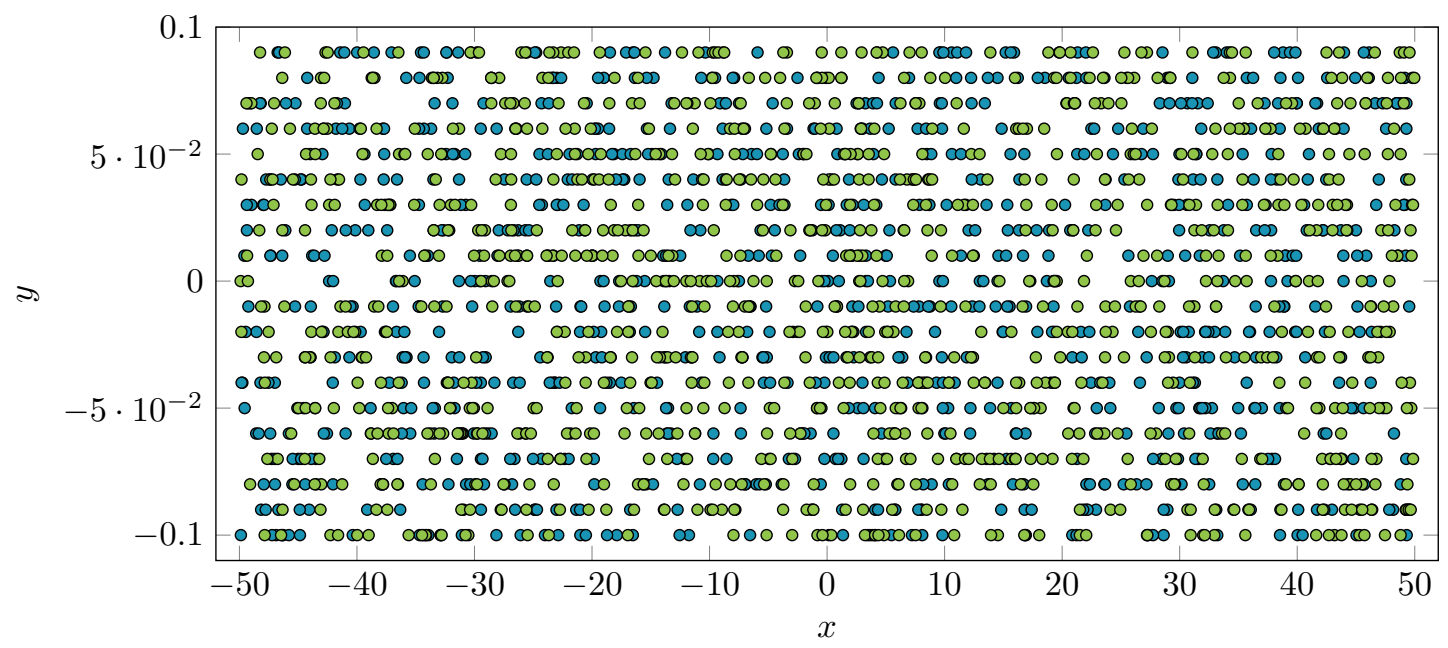

(a) Vortices arranged in UUNR configuration convecting in straight lines resembling streamlines without any body of influence ; green symbols indicate negative (clockwise flow), and blue symbols indicate positive (counterclockwise flow) circulation, respectively

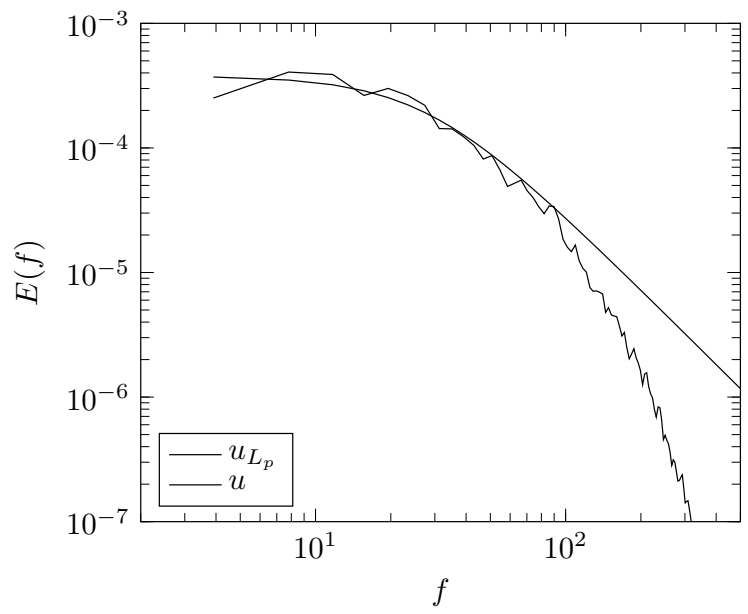

(b) longitudinal spectrum $u_{r m s} / U=0.12861208, \lambda=$ $0.0057175, N=10000$, Mexican - hat,$\Gamma_{r m s}=$ $1.0, \rho_{r m s}=0.1$

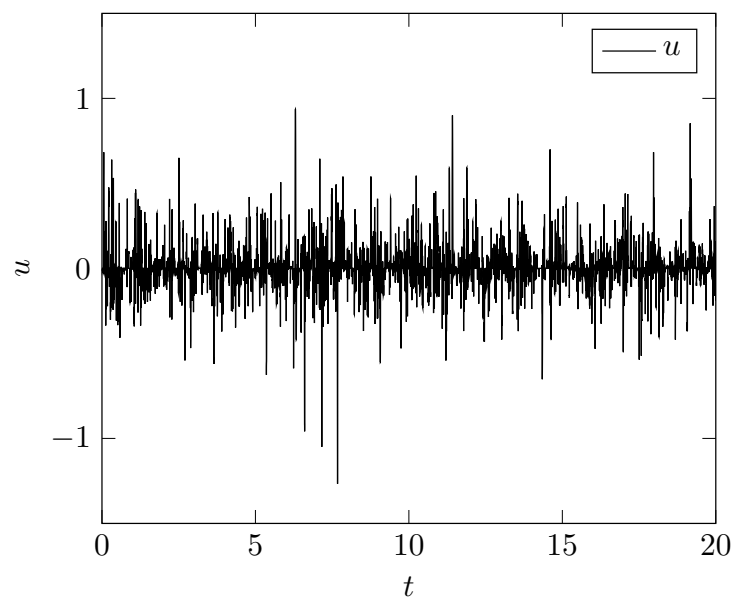

(d) velocity component $u$ fluctuations with time

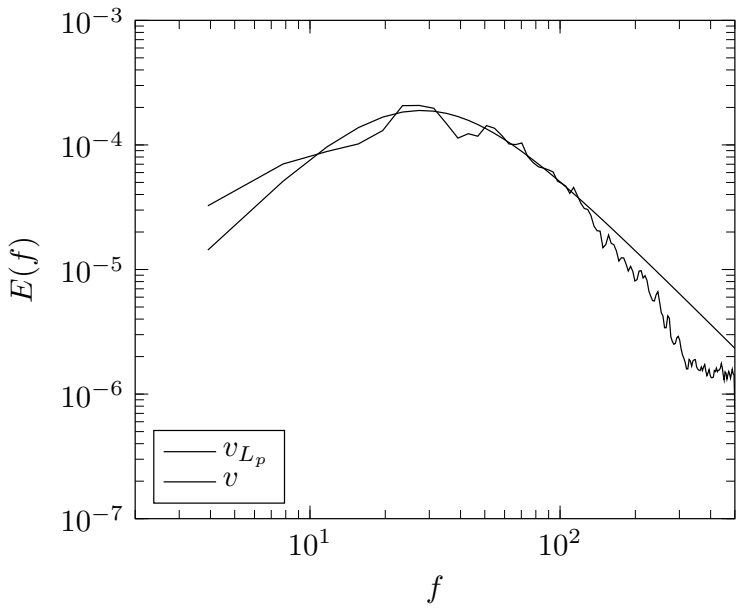

(c) transverse spectrum $u_{r m s} / U=0.12861208, \lambda=$ $0.0057175, N=10000$, Mexican - hat,$\Gamma_{r m s}=$ $1.0, \rho_{r m s}=0.1$

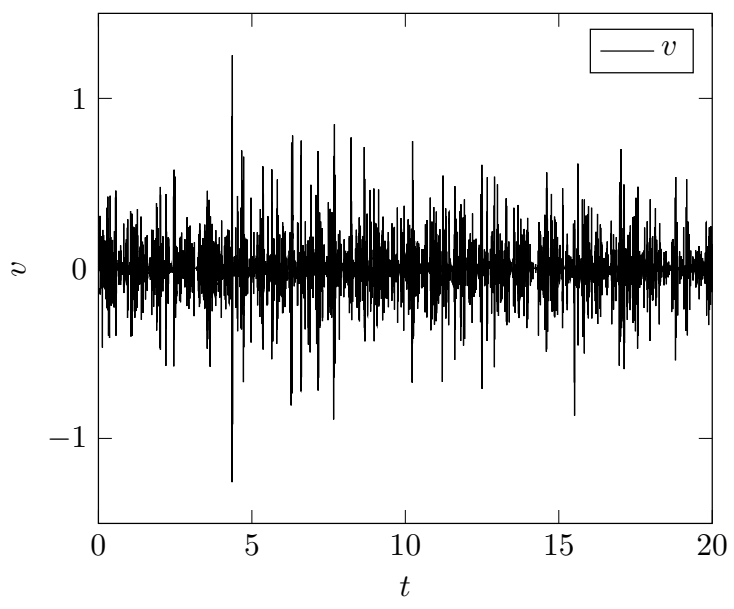

(e) velocity component $v$ fluctuations with time

Figure 4.11: $(a-b)$ spectral density of velocity induced by 10000 particles convecting in straight lines resembling streamlines with no body of influence in the domain; $(c-d)$ induced velocity fluctuations 
domain is 100 chord lengths long and 0.2 chord lengths high. The parameters controlling the vortex distributions are given in Table. (4.4). Particles do not share velocity influence among themselves, i.e. a particle is not influenced by the induced velocity field of the remaining parties and vice versa in the vortex window. At $t=0$, the vortex window is stationed at a point which is in farfield such that there is no induced velocity is experienced at the observation point, $O$ at $(0,0)$. The clustered vortices are arranged in the vortex window which starts to convect with $U=5 \mathrm{~m} / \mathrm{s}$, aligned with the $x$-axis at $t=t_{s}$. As the window approaches towards $O$, the observation point starts to experience induced velocities from all the vortex particles confined in the window. The determined length requires a vortex to travel for a duration of $(100 \mathrm{~m}) /(5 \mathrm{~m} / \mathrm{s})=20 \mathrm{~s}$, which is sufficiently long to conduct a statistical analysis. There is a good agreement in the low-frequency range for both longitudinal and transverse spectra, while at higher frequencies the longitudinal velocity spectra obtained is seen to decay energy as shown in Fig. ( $\underline{4.11 b)}-(\underline{4.11 c})$.

The main objective of this investigation is to construct a turbulent velocity signal in which:

i) the convection of the vortices is streamlined,

ii) and it corresponds to the statistics of two-dimensional atmospheric turbulence governed by the Liepmann spectra.

In the next section, this 'prepared' turbulent signal is used as a reference to create a vortexsound database for a NACA0012 airfoil by generating unsteady loading over its surface for dipole noise radiation.

\subsubsection{Acoustic radiation due to a turbulent signal}

The position of the injection line is kept unchanged at $x_{o}=-3.0$, and the vortices are released from 20 points arranged uniformly with an equal distance of 0.01 between them. This arrangement is chosen to accommodate statistically optimised velocity signal generated using Mexican-hat waveform mollified vortex particles in the last section (sec. 4.2.4). The distribution governing the distribution for vortex strength is normal with $\Gamma_{r m s}=1.0$, and for vortex, radius is Rayleigh with scaling parameter $\sigma_{R}=0.1$. The radius values are limited to $\{0.1,0.2,0.3,0.4,0.5\}$, nevertheless, the strength is freely governed by the normal distribution, which allows the strength variable to have any real number value. This is possible due to Eq. $\left(\underline{4.12}_{-}^{8}\right.$, which can estimate the sound-induced by a vortex, while interacting with NACA0012 airfoil, of any strength provided that the vortex is of the same size group. The chord line of the airfoil is parallel to the streamwise direction, and its leading-edge is oriented opposite to the flow direction. The principle of database matrix construction is similar to the one used in the previous section for a single streamline, whereas, here the number of streamlines are increased to 20 to accommodate 10000 vortices.

\subsubsection{Vortex-sound database}

Two key parameters, $\Gamma_{r m s}$ and $\sigma_{R}$, regulate the vortex-sound database making it suitable to generate statistical velocity signals based on $T u$ and $\lambda$. Furthermore, each database applies

\footnotetext{
${ }^{8}$ This equation is airfoil/body dependent, meaning the written equation only works for the NACA0012 airfoil placed at an angle of attack of zero degrees.
} 


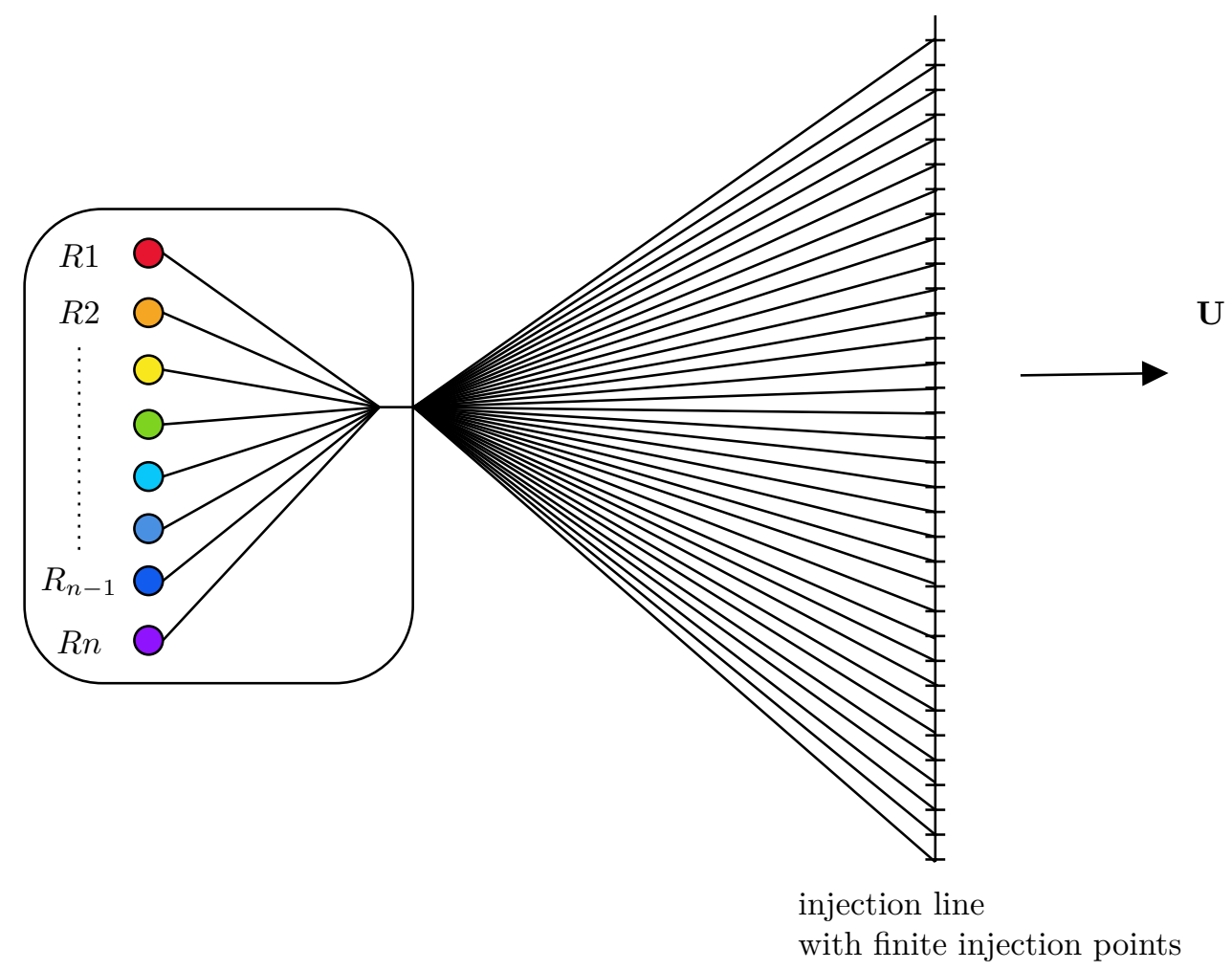

Figure 4.12: all injection points

to only one airfoil. As shown in the sec. (3.6.6.4), $\Gamma_{r m s}$ and $\sigma_{R}$ determine the turbulent intensity, $T u$, and length scale, $\lambda$, in vortex distribution. There exists no problem in reflecting a change in acoustic spectra due to a change in $\Gamma_{r m s}$, thus having the flexibility to consider any value for gamma for the vortex distribution. However, the distribution for radius does not allow such flexibility while playing around with the Rayleigh scaling parameter. The database becomes inefficient if the radius value is chosen beyond the specified values. For example, the database developed in the previous section can only provide acoustic data for five radii, and if the selected radius value is not one of those five radii, then using this database for that vortex is trivial. To overcome this, there are two ways:

i) The values of the radius should be chosen from the specified values,

ii) Adding some more values to the specified values of radii so that the database can be accessed for more values of radii.

In the present work, the first of the measures given above is adopted, i.e. the radius values are restricted to be from the predetermined five values. This can be visualised for 20 injection points in Fig. (․112).

Strength sample space Normal distribution

Radius sample space Rayleigh distribution with sample space limited to $\{0.1,0.2,0.3,0.4,0.5\}$ 


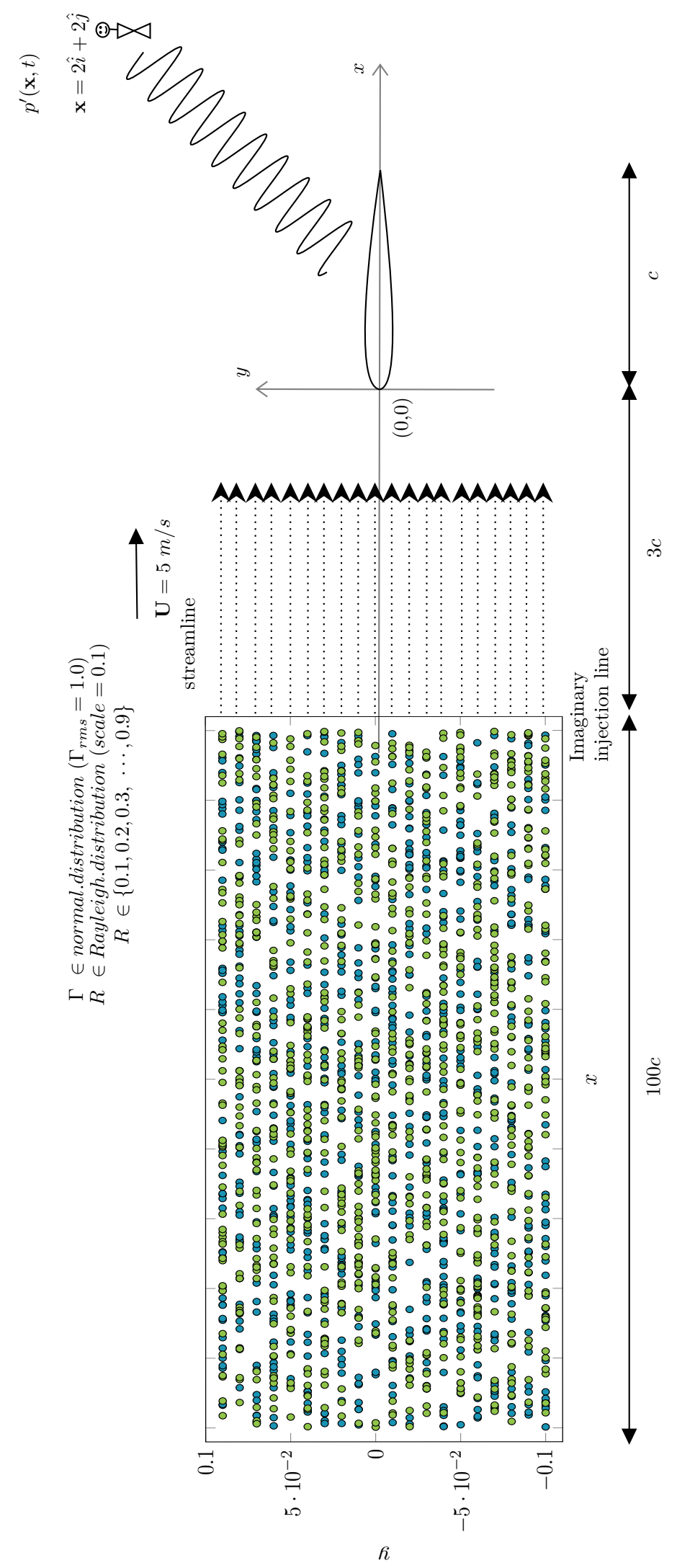

Figure 4.13: Implementation of the method 
Injection point sample space The injection points are uniformly distributed on the injection line with an equal distance of $\Delta y_{o}=0.01$ yielding 20 values, $y_{o} \in\{-0.1,-0.09, \ldots, 0.09\}$. The entire approach is visualised in Fig. (4.13).

\section{Implementation}

i) Steady-state flow analysis of NACA 0012 airfoil is carried out using the solver developed in chapter-2. Flow characteristics around the airfoil are solved by dividing both the upper and lower side surfaces into 100 straight lines panels each, enforcing flow tangency and Kutta-condition over them. The airfoil is aligned at an angle of attack of zero degrees with the streamwise axis with the free stream velocity kept constant at $5 \mathrm{~m} / \mathrm{s}$. The calculated streamlines later function as pathlines on which the convection of the vortex particles takes place.

ii) This is followed by setting up injection points on the injection line. The injection line is perpendicularly raised three chord lengths before the leading-edge of the airfoil, which is located on the origin of the Cartesian plane facing against the streamwise direction. Twenty injection points are spaced equally along this line, symbolising the origin of streamlines.

iii) Once the coordinates of streamlines, originating from 20 injection points, are computed, it is now time to convect a reference vortex on all these streamlines. The reference vortex is a mollified vortex particle using a Mexican-hat waveform with no singularity at the centre, meaning that there is no infinite rotational velocity at the absolute centre. The speed of the convection is based on the background flow itself, i.e. the speed of the freestream which is $5 \mathrm{~m} / \mathrm{s}$, and the time step is $0.001 \mathrm{~s}$ leading to a sampling frequency size of $500 \mathrm{~Hz}$. The Turbulent Velocity signal developed in sec (4.2.4) contained 10000 vortices in a 100 chord lengths long vortex window. Hence the entire window must be entirely convected through the domain while creating a database which requires the solution to march in time 4200 times. The strength of reference vortex is fixed as 0.1 , and five values $\{0.1,0.2,0.3,0.4,0.5\}$ are used for the core radius. Therefore, for example, on a streamline commencing from $\left(x_{o}, y_{o}\right)=(-3.0,0.01)$, five exclusive convections take place with each convection have a different sized vortex.

iv) As soon as the vortex enters into the domain of interest, it begins to induce velocity at the collocation points on the airfoil panels. With each step marching forward in time, the distance between the vortex marching forward and the airfoil changes and results in a time-dependent induced velocity profile. After complete convection of the vortex, the induced velocity on the panels is plotted over time to find a fluctuating velocity signal. The fluctuating velocity signal sensed by each panel is now converted to fluctuating pressure with the help of unsteady Bernoulli's equation.

v) Finally, it is time to post-process this unsteady pressure using the frequency form of the Curle's equation. The listener is located in the far-field with its coordinates set at $(2.0,2.0)$. The dipole sound radiation from the time-dependent unsteady pressure induced on the surface of the airfoil from the convection of vortices of five different sizes from each streamline is stored in a three-dimensional matrix. 
vi) The developed database is shown as a three-dimensional matrix in the Fig. (4.14). Note: this database is only operating for a specific profile, NACA0012 in this case. Some parts of it may be useful in a case with another angle of attack or a different freestream velocity, but that is a matter of further case study.

The table below enumerates the values and details of all the parameters used to create the vortex-sound database above.

\subsubsection{How to use the vortex-sound database?}

The entire code is written in python which allows the user to save the matrix/array in numpy array format which can be used later in an independent code by simply inserting it as a library. In the above implementation, the database obtained can be used to predict the dipole noise radiation from the surface of NACA0012 airfoil for different $T u$ and $\lambda$.

\subsubsection{Virtual injection of 10000 particles}

At $\mathrm{t}=0$, all the injection points randomly select a value for strength and radius for the vortex from the database matrix, as shown in Fig. ( $\underline{4.9)}$ and stores the SPL spectrum into a temporary variable. Each streamline convects a total of 500 vortices and the principle of calculation of correct SPL due to each vortex with the time-delay correction is the same as for the single streamline injection as discussed in sec. $\underline{(4.2 .3 .1})$.

\subsubsection{D-3D Correction}

In order to compare the broadband predictions based on $2 \mathrm{D}$ simulations to the problem of sound radiation from a $3 \mathrm{D}$ wing of a finite span, additional corrections have to be added to the simulated 2D spectra. Ewert et al. [167] proposed a simple correction formula which translates the $2 \mathrm{D}$ spectra to a $3 \mathrm{D}$ one.

$$
S_{p p \mid 3 D}=\frac{\omega l_{z}(\omega) d}{2 \pi c_{o} R} S_{p p \mid 2 D},
$$

where $l_{z}(\omega)$ is the spanwise coherence length scale which is assumed to be smaller than the wingspan, $d$. $\mathrm{R}$ denotes denotes the distance from the wing trailing edge to the observer in the far-field which is larger than the span, i.e. $l_{z}<d<R$. The frequency dependence of the spanwise coherence length scale of surface pressure was derived by Amiet [166] to obey in the high-frequency limit a relationship $l_{z} \propto \omega^{-1}$. Experimental work reveals a relationship $l_{z}=C \cdot(U / \omega)$ with the constant being in range $C \simeq 2.1$. Thus, using this relationship, Eq. $\underline{(4.13)}$ becomes

$$
S_{p p \mid 3 D}=\frac{C}{2 \pi} \frac{L}{R} M_{\infty} \cdot S_{p p \mid 2 D}
$$

\subsection{Results and discussion}

An extensive vortex-sound database is created following the implementation protocols given above. The generated database is capable of reconstructing all three cases of Tu and Lambda 


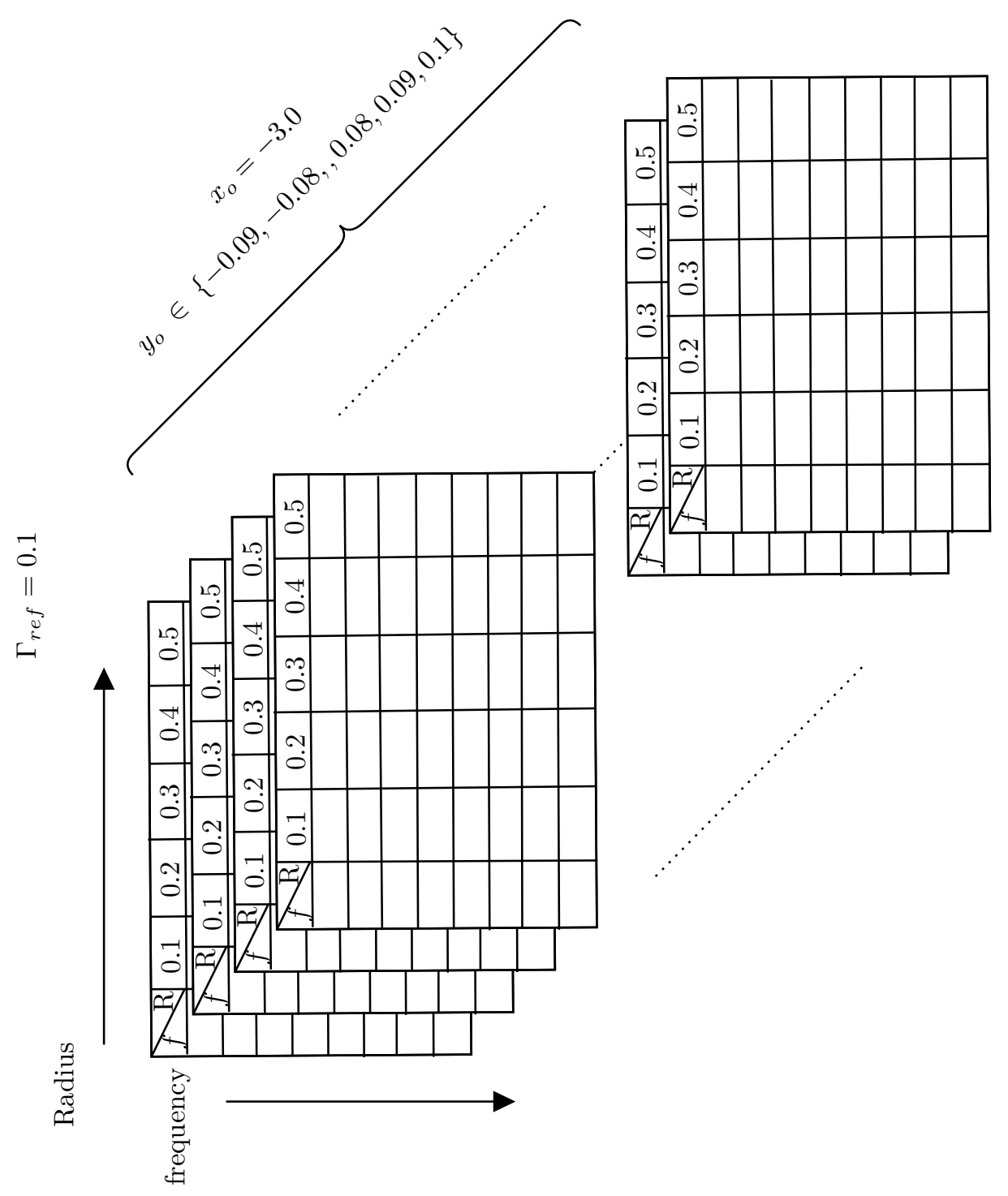

Figure 4.14: Multidimensional matrix 
given in Table. (3.6). With alterations such as considering a more comprehensive range of values of radius, $R$, and injection points, $y_{o}$, can enable more numerous $T u$ or $\lambda$ cases to be reconstructed. Table. (4.5) shows all the parameters that are employed to construct the database.

\subsubsection{Comparison with Amiet's analytical formulation}

The interaction noise generated by the isotropic homogeneous turbulent flow of $1.19 \%, 4.9 \%$, $13.2 \%$ turbulence intensity and $4.21 \mathrm{~mm}, 4.29 \mathrm{~mm}, 5.5 \mathrm{~mm}$ integral length scale, impinging on NACA0010 and NACA0012 is predicted using the vortex-sound database. The airfoils are located at the origin with their leading-edge coinciding with it and facing opposite to the streamwise direction. The noise is predicted at a single listener located at $\mathbf{x}=2 \widehat{i}+2 \widehat{j}$ in the Cartesian coordinate system. The PSD of sound pressure radiated by NACA0012 is shown in Fig. ( $\underline{4.15})$, and by NACA0010 in Fig. ( $\underline{4.16})$ against Amiet's flat plate analytic theory [19] and Amiet's analytic theory with Gershfeld's thickness correction [24] for chord length, $c_{a}$ of $1 \mathrm{~m}$, span, $d$, of $1 \mathrm{~m}$ at $U=5 \mathrm{~m} / \mathrm{s}$. The comparison shows the corrected spectra ( $2 \mathrm{D}$ to 3D) obtained using the vortex-sound database are in good agreement with Amiet's formula with Gershfeld's correction for all the three cases of $T u$ and $\lambda$ for both airfoils. Since the method does not account for the feedback effects from the trailing edge vortices to the incoming vortices, the radiated noise spectra do not exhibit any oscillations due to the interference between the leading-edge and trailing-edge [ $\underline{52}, \underline{168}$.

The far-field spectral plots are presented in terms of the Sound Pressure Level spectrum SPL(f).

Table 4.5: Parameters involved in the interaction of 10000 vortices, injecting out of 20 points scattered uniformly on the injection line, with NACA0012 airfoil

\begin{tabular}{ll}
\hline Parameters & Values \\
\hline$x_{o}$ & -3.0 \\
$y_{o}$ & $\{-0.1,-0.09, \ldots, 0.09\}$ \\
Number of injection points & 20 \\
$\Gamma_{\text {ref }}$ & 0.1 \\
$\sigma_{R}$ & 0.1 \\
Vortex production rate & 110 \\
Number of vortex particles & 10000 \\
Vortex type & Mexican-hat waveform \\
Airfoil & NACA0012 \\
Number of panels & 200 \\
Panel singularity & Source and vortex \\
$U$ & $5 m / s$ \\
$\alpha$ & $0^{\circ}$ \\
Time step, $\Delta t$ & 0.001 \\
Number of time steps & 4200 \\
Frequency sampling, $F_{s}$ & $1000 \mathrm{~Hz}$ \\
\hline
\end{tabular}




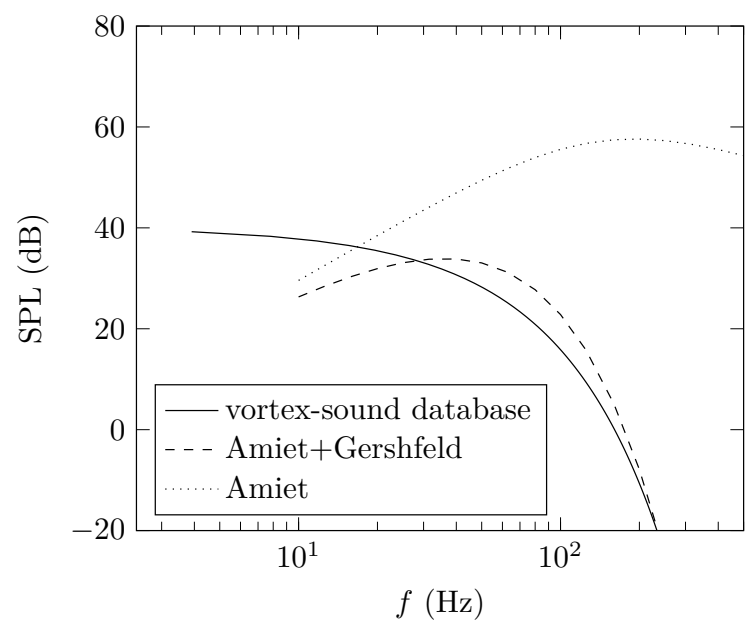

(a) $u_{r m s} / U=0.0119, \lambda=0.00421, N=$ 10000, Mexican - hat, $\Gamma_{r m s}=0.1, \rho_{r m s}=0.1$

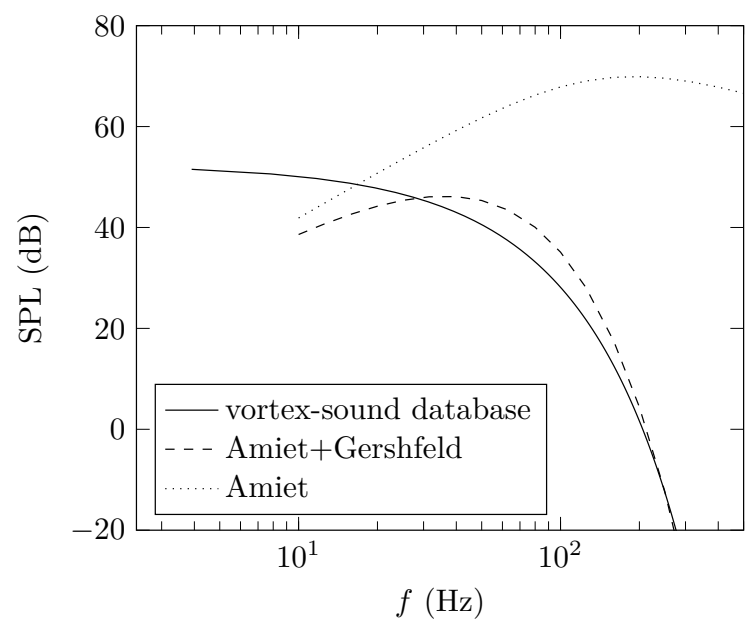

(b) $u_{r m s} / U=0.049, \lambda=0.00429, N=$ 10000, Mexican - hat, $\Gamma_{r m s}=0.4, \rho_{r m s}=0.1$

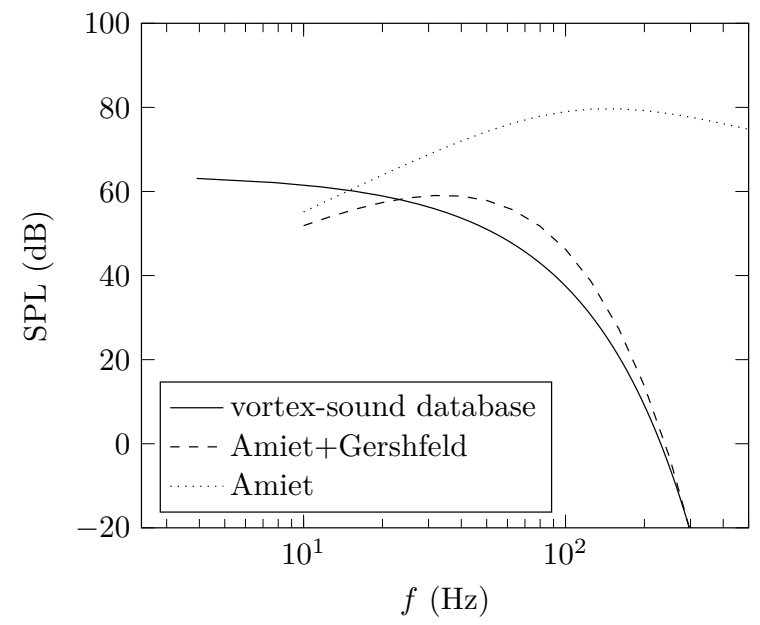

(c) $u_{r m s} / U=0.132, \lambda=0.0055, N=$ 10000, Mexican - hat, $\Gamma_{r m s}=1.0, \rho_{r m s}=0.1$

Figure 4.15: Sound Pressure Level (SPL) due to 10000 vortices on NACA0012 airfoil 


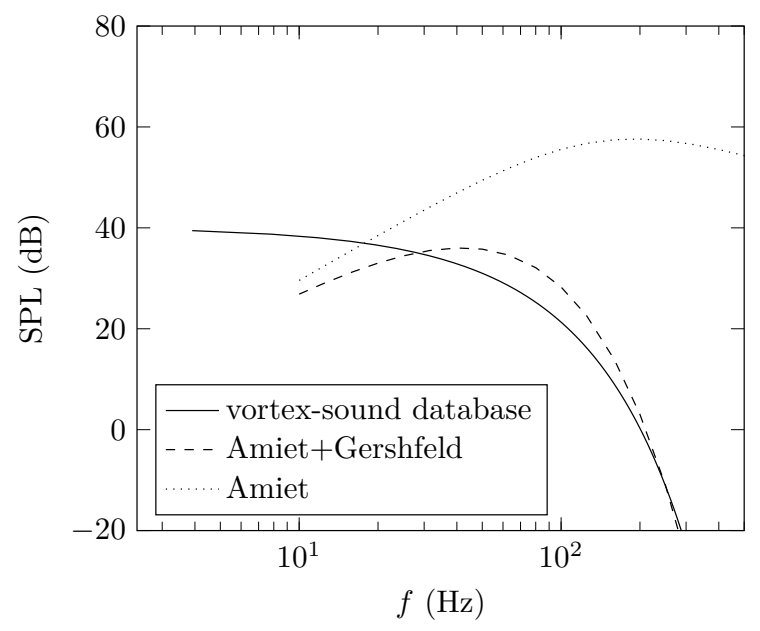

(a) $u_{r m s} / U=0.0119, \lambda=0.00421, N=$ 10000, Mexican - hat, $\Gamma_{r m s}=0.1, \rho_{r m s}=0.1$

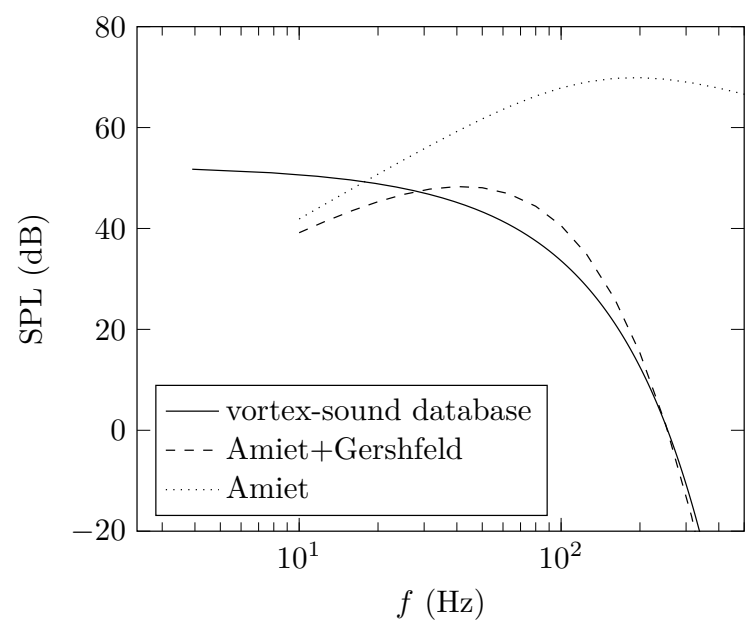

(b) $u_{r m s} / U=0.049, \lambda=0.00429, N=$ 10000, Mexican - hat, $\Gamma_{r m s}=0.4, \rho_{r m s}=0.1$

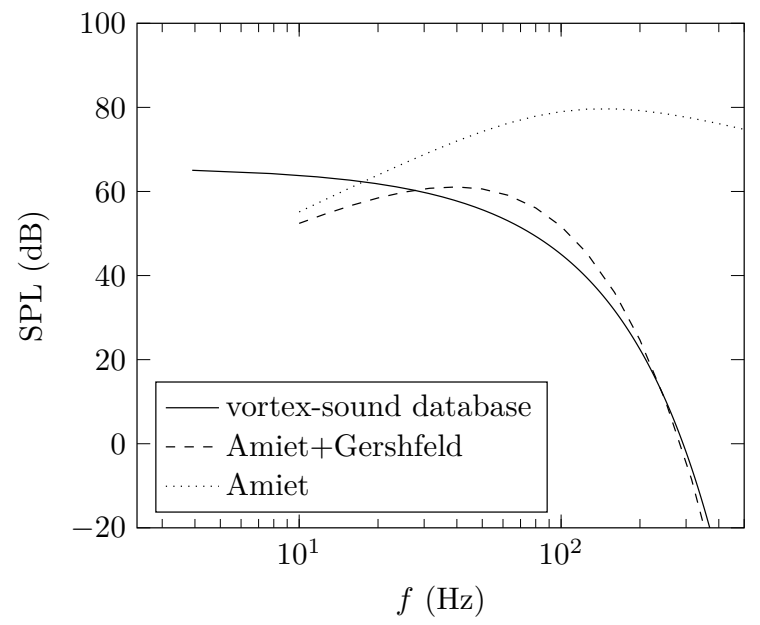

(c) $u_{r m s} / U=0.132, \lambda=0.0055, N=$ 10000, Mexican - hat, $\Gamma_{r m s}=1.0, \rho_{r m s}=0.1$

Figure 4.16: Sound Pressure Level (SPL) due to 10000 vortices on NACA0010 airfoil 


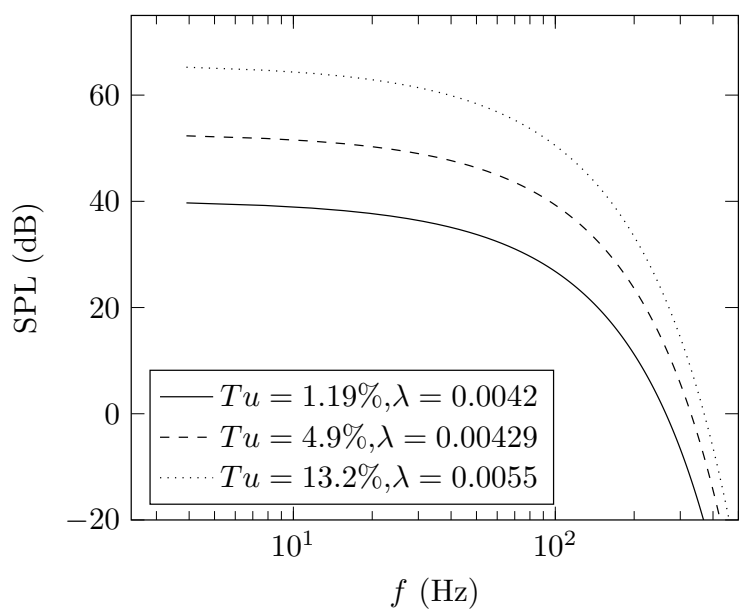

(a) NACA0008

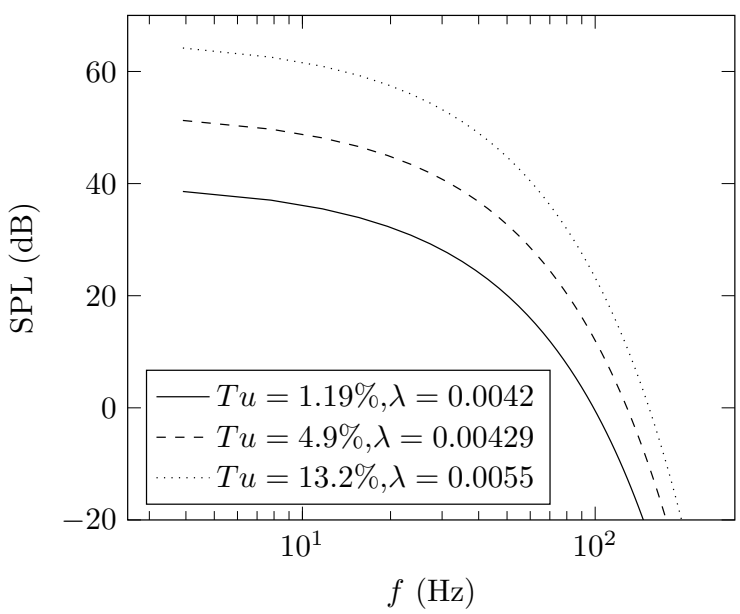

(b) NACA0018

Figure 4.17: SPL variations with Tu and $\lambda$

\subsubsection{Effect of $T u$ and $\lambda$}

In this study, NACA0008, a thin and NACA0018, a thick airfoil is introduced in three flow fields characterised by different turbulence intensities and length scales, and radiated loading noise due to unsteady pressure fluctuations are predicted using the vortex-sound database. The chord length $c_{a}$ is $1 \mathrm{~m}$ with the mean flow speed of $5 \mathrm{~m} / \mathrm{s}$. As expected, the flow field with higher turbulent intensity induces greater fluctuations over the surfaces of both airfoils. Fig. (4.17a) shows the PSD of the radiated noise by NACA0008 and Fig. (4.17b) shows the noise due to NACA0018.

\subsubsection{Effect of thickness}

This section examines the ability of vortex-sound database method to predict the effect of thickness on the loading noise effectively. Sound Pressure Level (SPL) is plotted against the frequency for airfoils with varying thickness from $8 \%$ to $18 \%$ on a non-cambered NACA00 series for a mean flow velocity of $5 \mathrm{~m} / \mathrm{s}$. At all three $T u$ and $\lambda$, Fig. (4.18) shows that sound pressure level decreases with increasing thickness, which is consistent with earlier studies $[33$, $\underline{24}, \underline{169}, \underline{165}, \underline{63}$.

\subsubsection{Effect of angle of attack}

The effect of angle of attack on the generation of loading noise is analysed by investigating three different airfoils (NACA0008, NACA0012m and NACA0018) at three different angles of attack of $0^{\circ}, 5^{\circ}$, and $10^{\circ}$. Fig. (4.19a) shows the sound pressure levels due to NACA0008 at three different angles of attack $\left(\alpha=0^{\circ}, 5^{\circ}\right.$, and $\left.10^{\circ}\right)$ in a flow field characterised by a turbulence intensity of $1.19 \%$ and length scale of $4.9 \mathrm{~mm}$. Similarly, Fig. (4.19b) shows the SPL due to NACA0012, and Fig. $(\underline{4.19 \mathrm{c})}$ due to NACA0018. It is evident from the plots that the effect of AOA on airfoil-turbulence interaction noise is negligible. The results are consistent with the findings of Juknevicius and Chong [170], Devenport, Staubs, and Glegg [48], and Moreau, Roger, and Jurdic [42] where the effect of angle of attack is found weak in the presence of isotropic turbulence. 


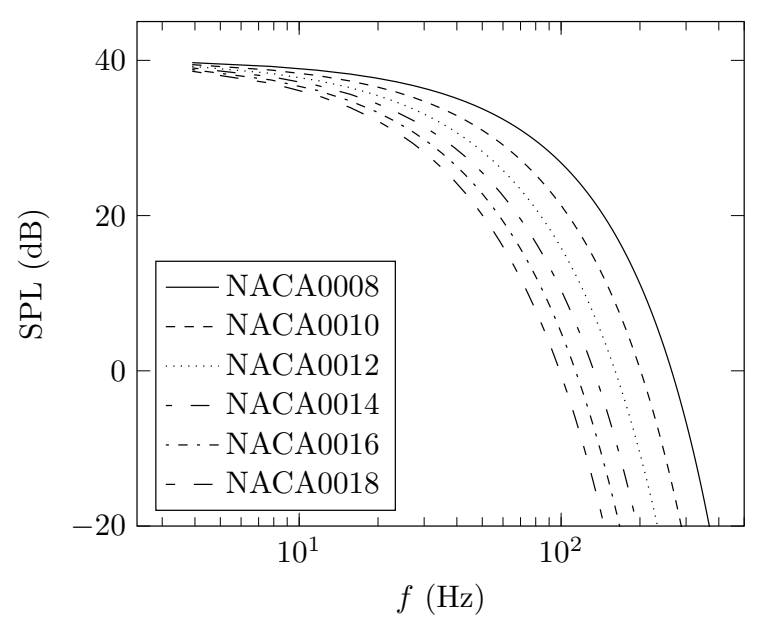

(a) $u_{r m s} / U=0.0119, \lambda=0.00421, N=$ 10000, Mexican - hat, $\Gamma_{r m s}=0.1, \rho_{r m s}=0.1$

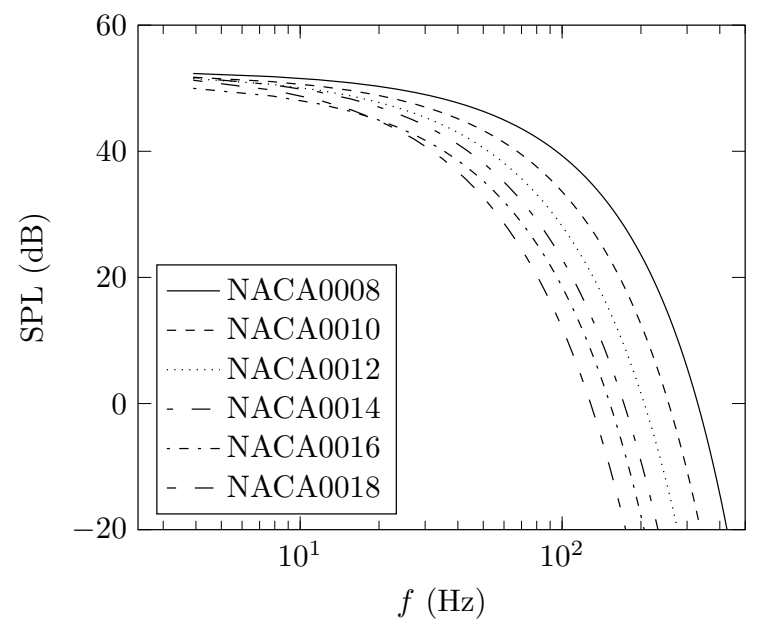

(b) $u_{r m s} / U=0.049, \lambda=0.00429, N=$ 10000, Mexican - hat $, \Gamma_{r m s}=0.4, \rho_{r m s}=0.1$

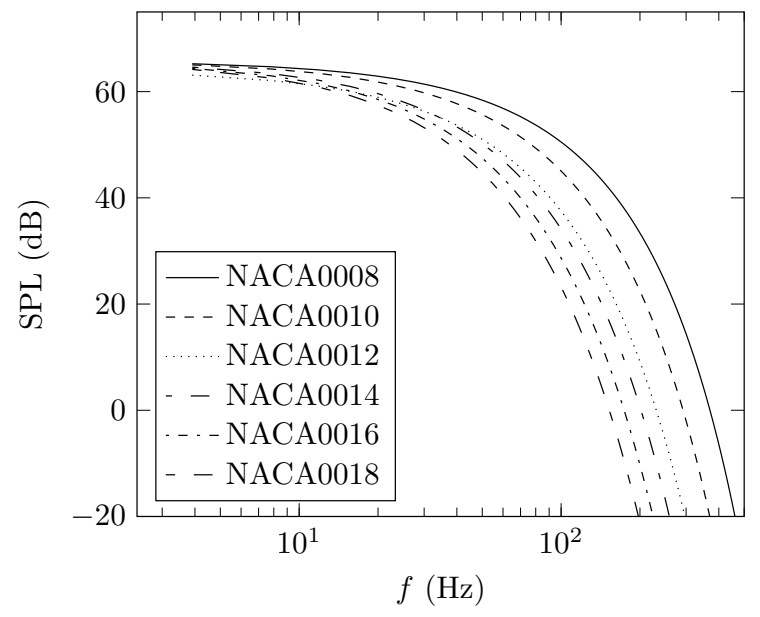

(c) $u_{r m s} / U=0.132, \lambda=0.0055, N=$ 10000, Mexican - hat, $\Gamma_{r m s}=1.0, \rho_{r m s}=0.1$

Figure 4.18: Sound Pressure Level (SPL) due to 10000 vortices airfoil of varying thickness 


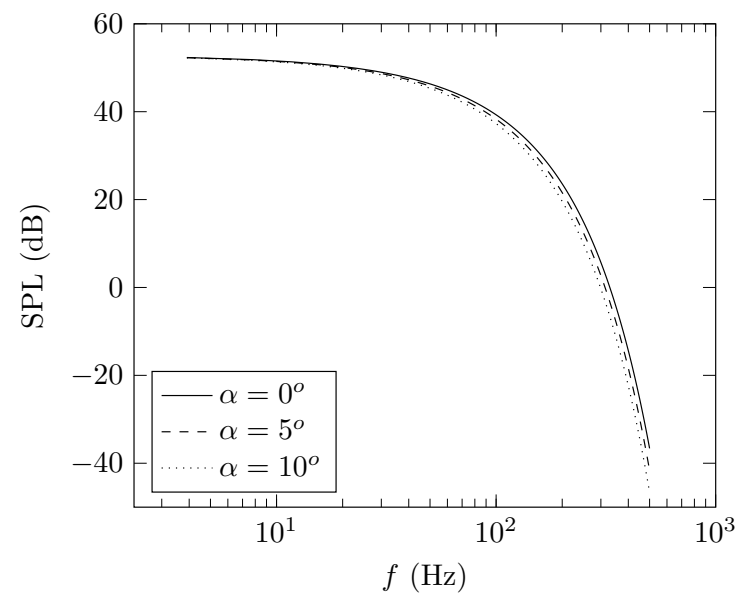

(a) NACA0008

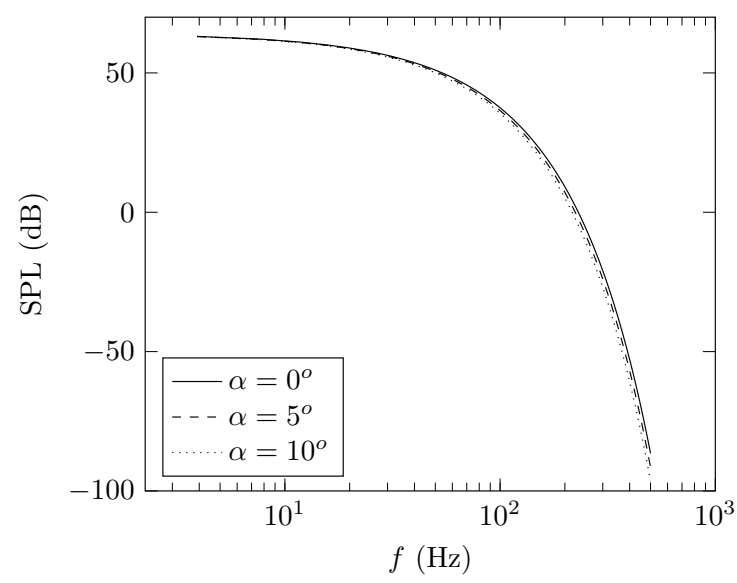

(b) NACA0012

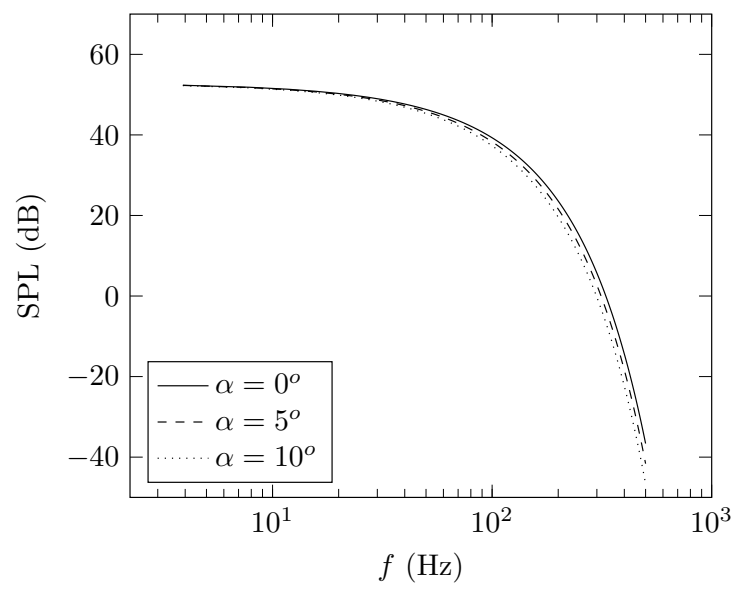

(c) NACA0018

Figure 4.19: Sound Pressure Level (SPL) due to 10000 vortices airfoil of varying angle of attack 



\section{5}

Conclusion

The main objective of this thesis is to propose a mathematical model which can efficiently predict leading-edge noise in time-domain. The method can effectively predict the effect of both geometric attributes (thickness, angle of attack, etc.) and flow field attributes (turbulence intensity, length scale, flow speed) on the noise generation.

\subsection{General conclusions}

i) The thesis gives a detailed description of the techniques practised to calculate the airfoilturbulence interaction noise and also suggests that it is possible to optimise the airfoil based on acoustic radiation. Shape optimisation of the airfoil to manipulate the aerodynamic characteristics already exists in numerical techniques, but the requirement for highresolution in grid-based methods in aeroacoustics makes the entire process of shape optimisation computationally expensive.

ii) That is why vortex-method is used instead of conventional grid-based methods to solve this problem. Generated loading-noise is predicted in the far-field due to unsteady-loading on the surface of the airfoil induced by atmospheric turbulence using vortex-method and FW-H in two dimensions.

iii) Using the stream function formulation, the flow-field around the airfoil is split into three parts: the first one for the vortex sheet distribution on the surface of the airfoil, the second one for the vorticity field due to atmospheric turbulence, and the third one for the velocity field at infinity. The last two are obtained with the help of the panel method, and a new approach is proposed in Chapter-3 for the first one.

iv) The above-mentioned panel method is developed in Python for this research work. The code is based on the algorithm and physics governing the incompressible and inviscid flow given in classical texts by Katz and Plotkin [85] and Cebeci et al. [91]. The developed system is capable of solving both steady and unsteady flow-fields as evidently shown 
in sec. $(2.7)$, which discusses and validates the results from steady and unsteady flow investigations.

v) The singularity problem associated with point vortex, the most basic unit of vortex methods, is solved with the help of kernel mollification. Vortex particles based on Gaussian and Mexican-hat waveform have been used to create the first streamfunction, $\psi_{\omega}$. This thesis demonstrates how these two mollified vortices mollify non-physical induced velocities near the centre of a point-vortex. This is followed by the statistical analysis of the induced velocity by a convecting single-particle (Gaussian and Mexican-hat particles separately), which showed that circulation strength, $\Gamma$, and radius, $R$, are the main attributes that govern the nature of the velocity field induced by these particles.

vi) The stream function formulation allows the application of inviscid panel method to account for the calculation of background flow which enables to compute the streamlines around the airfoil efficiently and quickly. The primary purpose of getting these streamlines is to construct the pathlines for the vortex particles that make up the streamfunction for vorticity present in the incoming disturbance. Incoming vortices do not interact with each other, producing frozen turbulence. Due to the entire system being linear, the velocities induced by all the particles at one point can be superimposed to find the total induced velocity at a point in the domain of interest.

vii) Another parameter, position, has been added with two parameters, strength and radius, controlling the velocity induced by the vortex particles. The three parameters are random in nature, and as a result, they are considered stochastic variables in this thesis. Standard probability density functions (PDF) can describe these stochastic variables. Stochastic variables of 200, 400, and 800 particles (Gaussian and Mexican-hat, both individually) are then distributed in uniform, normal, and Rayleigh fashion. The statistical investigation of induced velocity due to particles led to a conclusion that

(a) a uniform distribution is fit for Spatial Coordinates,

(b) a normal distribution is suitable for the circulation strength,

(c) and a Rayleigh distribution is appropriate for the radius

of the vortex particles to reconstruct a velocity signal which exhibits the statistics of two-dimensional turbulence represented by Liepmann spectra.

viii) Mexican-hat particles are chosen over the Gaussian particles due to their ability to produce the desired velocity spectra frequently.

ix) Five differently sized, $R \in\{0.1,0.2,0.3,0.4,0.5\}$, vortex particles with the same circulation strength, $\Gamma$, of 0.1 are released from 20 points on a vertical line perpendicular to the chord line. This vertical line is called the injection line, which allows the passage of vortex particles into the domain of interest and is kept three chord lengths upstream of the airfoil. The particles are convected for a total time of $20 \mathrm{~s}$ with the free stream velocity of $5 \mathrm{~m} / \mathrm{s}$. The sound pressure level due to fluctuating pressure induced by every vortex on the airfoil surface is calculated and stored in a matrix. The variation in the vortex strength (from 
0.1 to any real number) is reflected in the corresponding sound pressure levels in terms of vertical shift of the plot which allows the generated matrix to be able to be useful for all the possibilities of strength and radius.

x) Two separate vortex-sound databases for NACA0010 and NACA0012 airfoil are generated from which three isotropic homogeneous turbulent inflow signals (characterised by $1.19 \%$, $4.9 \%, 13.2 \%$ turbulence intensity and $4.21 \mathrm{~mm}, 4.29 \mathrm{~mm}, 5.5 \mathrm{~mm}$ integral length scale) are created. By introducing these three signals on the airfoil at $\alpha 0$, the sound pressure level was determined, which after 3D correction appeared to be a good match with the results obtained by Amiet's analytic formula.

\subsection{Advantages}

The main advantages of using vortex-sound database are:

i) The proposed approach is not dependent on grids, which makes it less expensive than the mesh-based methods which are usually computationally expensive methods.

ii) Vortex databases can be created for infinite injection points and radius values, allowing the possibility to generate velocity signals of infinite combinations of turbulent intensity and length scales.

iii) The method is computationally inexpensive compared to classical vortex methods since the effect due to particles are precomputed, stored in a matrix. The matrix can be called as a library while predicting the noise from a specific airfoil.

iv) The method can be used not only for airfoil, but also for other bodies of interest in aerodynamics and acoustics, such as cylinders.

\section{$5.3 \quad$ Future scope}

The recommendations for future work are listed below:

i) To extend the model to $3 \mathrm{D}$ to understand the noise-reduction mechanisms more clearly and precisely.

ii) To consider other options (Morlet mother waveform) for the kernel mollification of the vortex particles, which can enable to reconstruct velocity signals with desired statistics in higher frequency range.

iii) To study the stochastic variables with a wider range of probability distribution functions to find other possible combinations for the variables.

iv) To extend the model for complex rotating turbomachines.

Fig. (5.1) shows the construction and implementation of the vortex-sound database model for an airfoil. The first step is to calculate the background flow, followed by convection of optimised vortex particles in streamlines around the airfoil, and also storing the acoustic 
radiation due to the interaction of each vortex particle with the airfoil. The next step is then to just superimpose the acoustics signals to arrive at the total sound pressure level at a specific point in the far-field due to airfoil-turbulence interaction noise. 

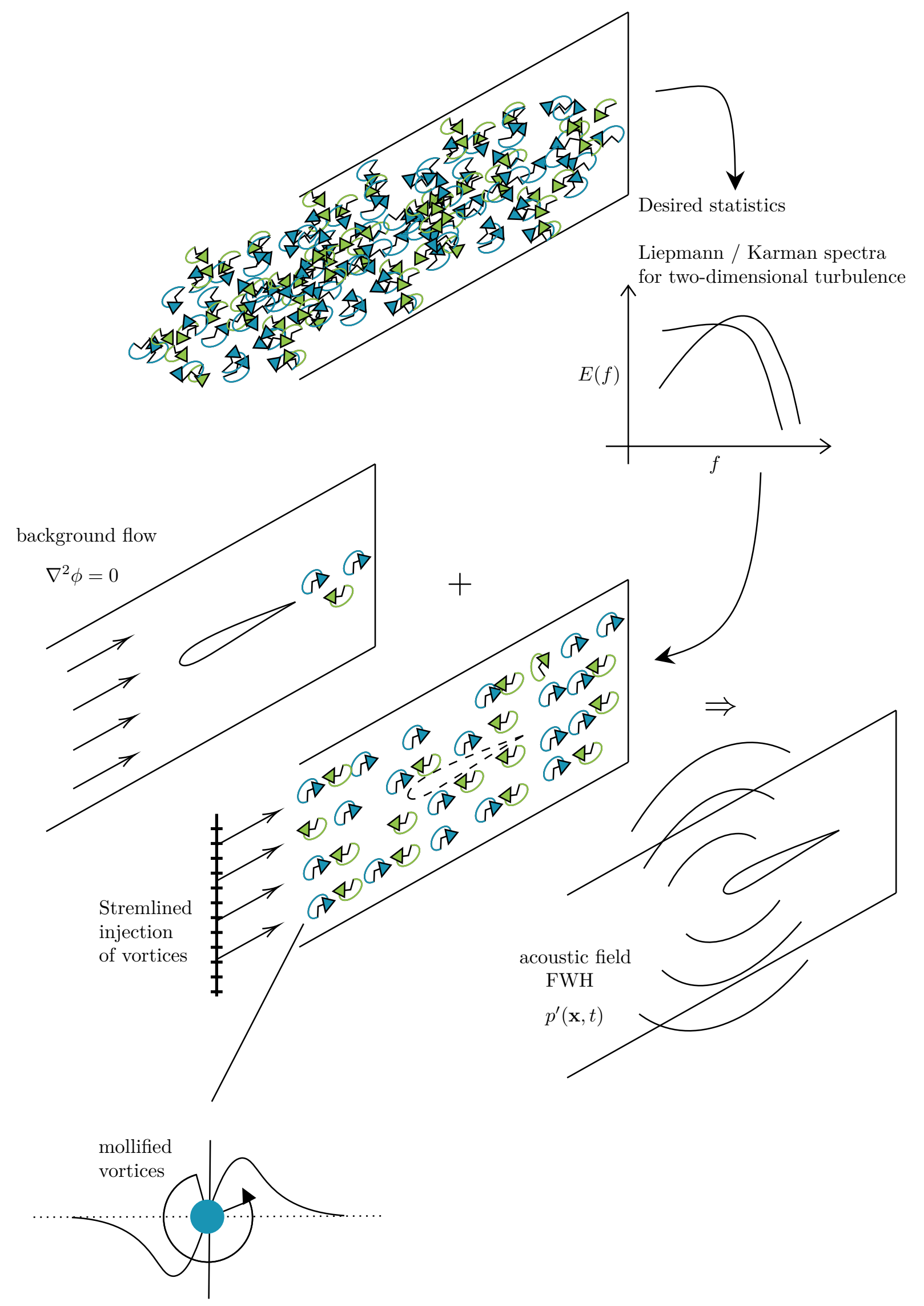

Figure 5.1: Construction and implementation of the vortex-sound database model 



\section{References}

[1] I. J. Sharland. "Sources of Noise in Axial Flow Fans". In: Journal of Sound and Vibration 1.3 (1964), pp. 302-322. ISSN: 00014966. DOI: 10.1121/1.1977649.

[2] John D. Anderson. "Ludwig Prandtl's Boundary Layer". In: Physics Today 58.12 (Dec. 2005), pp. 42-48. ISSN: 0031-9228. DOI: 10.1063/1.2169443.

[3] L. Prandtl. "Über die Entstehung von Wirbeln in der idealen Flüssigkeit, mit Anwendung auf die Tragflügeltheorie und andere Aufgaben". In: Vorträge aus dem Gebiete der Hydround Aerodynamik (Innsbruck 1922). Berlin, Heidelberg: Springer Berlin Heidelberg, 1922, pp. 18-33. DOI: 10.1007/978-3-662-00280-3_2.

[4] Walter Birnbaum. "Das ebene Problem des schlagenden Flügels". In: ZAMM - Zeitschrift für Angewandte Mathematik und Mechanik 4.4 (1924), pp. 277-292. DOI: 10.1002/ zamm.19240040401.

[5] H Wagner. "Dynamischer auftrieb von tragflügeln." In: ZAMM - Zeitschrift für Angewandte Mathematik und Mechanik 5.17 (1925).

[6] Ülgen Gülçat. Fundamentals of modern unsteady aerodynamics, second edition. Singapore: Springer Singapore, 2016, pp. 1-395. ISBN: 9789811000188. DOI: $10.1007 / 978-$ 981-10-0018-8.

[7] A. M. Kuethe and C. Y. Chow. Foundations of aerodynamics: bases of aerodynamic design. 3rd ed. 1976.

[8] John Anderson. Fundamentals of Fundamentals of. Vol. 5. 2013, pp. 447-469. ISBN: 0814471323.

[9] Theodore Theodorsen. General theory of aerodynamic instability and the mechanism of flutter. Tech. rep. No. 496. 1935, p. 24. DOI: 10.1017/CB09781107415324.004. arXiv: arXiv: $1011.1669 \mathrm{v} 3$.

[10] H. G. Küssner. "Zusammenfassender Bericht über den instationären Auftrieb von Flügeln." In: ZAMM - Zeitschrift für Angewandte Mathematik und Mechanik (1936).

[11] T von Kàrmàn and W.R. Sears. "Airfoil Theory for Non-Uniform Motion". In: Journal of the Aeronautical Sciences (Institute of the Aeronautical Sciences) 5.10 (1938), pp. 379390. ISSN: 1936-9956. DOI: $10.2514 / 8.674$.

[12] WR Sears. "A systematic presentation of the theory of thin airfoils in non-uniform motion". PhD thesis. California Institute of Technology, 1938. 
[13] H. W. Liepmann. "Extension of the Statistical Approach to Buffeting and Gust Response of Wings of Finite Span”. In: Journal of the Aeronautical Sciences 22.3 (Mar. 1955), pp. 197-200. ISSN: 1936-9956. DOI: 10.2514/8.3305.

[14] H. S. Ribner. "Spectral Theory of Buffeting and Gust Response: Unification and Extension". In: Journal of the Aeronautical Sciences 23.12 (Dec. 1956), pp. 1075-1077. ISSN: 1936-9956. DOI: $10.2514 / 8.3733$.

[15] "On sound generated aerodynamically I. General theory". In: Proceedings of the Royal Society of London. Series A. Mathematical and Physical Sciences 211.1107 (Mar. 1952), pp. 564-587. ISSN: 2053-9169. DOI: 10.1098/rspa.1952.0060.

[16] "On sound generated aerodynamically II. Turbulence as a source of sound". In: Proceedings of the Royal Society of London. Series A. Mathematical and Physical Sciences 222.1148 (Feb. 1954), pp. 1-32. ISSN: 2053-9169. DOI: 10.1098/rspa. 1954. $\underline{0049}$.

[17] N. Curle. "The influence of solid boundaries upon aerodynamic sound". In: Proceedings of the Royal Society of London. Series A. Mathematical and Physical Sciences 231.1187 (Sept. 1955), pp. 505-514. ISSN: 2053-9169. DOI: 10.1098/rspa.1955.0191.

[18] P. E. Doak. "Acoustic radiation from a turbulent fluid containing foreign bodies". In: Proceedings of the Royal Society A: Mathematical, Physical and Engineering Sciences 254.1276 (1960). DOI: 10.1098/rspa.1960.0010.

[19] R. K. Amiet. "Acoustic radiation from an airfoil in a turbulent stream". In: Journal of Sound and Vibration 41.4 (1975), pp. 407-420. ISSN: 10958568. DOI: 10.1016/S0022$\underline{460 X(75) 80105-2 .}$

[20] H Lamb. "Kirchhoff's method". In: Hydrodynamics. 6th ed. Dover Publications, 1932, p. 501.

[21] H. Atassi and G. Hamad. "Sound generated in a cascade by three-dimensional disturbances convected in a subsonic flow". In: AIAA, Aeroacoustics Conference 1 (1981). DOI: doi: 10.2514/6.1981-2046.

[22] Marvin E. Goldstein. Aeroacoustics. McGraw-Hill International Book Co, 1976, p. 293. ISBN: 0070236852.

[23] Matthew R. Myers and E. J. Kerschen. "Influence of camber on sound generation by airfoils interacting with high-frequency gusts". In: Journal of Fluid Mechanics 353.-1 (June 1997), pp. 221-259. ISSN: 00221120. DOI: 10.1017/S0022112097007349.

[24] J Gershfeld. "Leading edge noise from thick foils in turbulent flows". In: Journal of the Acoustical Society of America 116.3 (2004), pp. 1416-1426. ISSN: 00014966. DOI: 10.1121/1.1780575.

[25] R. W. Paterson and R. K. Amiet. "Acoustic Radiation and Surface Pressure Characteristics of an Airfoil due to Incident Turbulence". In: NASA Cr-2733 (1976), p. 571. ISSN: 0021-8669. DOI: doi: 10.2514/6.1976-571. 
[26] H. Posson, S. Moreau, and M. Roger. "On the use of a uniformly valid analytical cascade response function for fan broadband noise predictions". In: Journal of Sound and Vibration 329.18 (2010), pp. 3721-3743. ISSN: 10958568. DOI: $10.1016 / \mathrm{j}$.jsv 2010.03.009.

[27] S. A.L. Glegg. "The response of a swept blade row to a three-dimensional gust". In: Journal of Sound and Vibration 227.1 (Oct. 1999), pp. 29-64. ISSN: 0022460X. DOI: 10.1006/jsvi.1999.2327.

[28] Lorna J. Ayton and N. Peake. "On high-frequency sound generated by gust-aerofoil interaction in shear flow". In: Journal of Fluid Mechanics 766 (Mar. 2015), pp. 297-325. ISSN: 0022-1120. DOI: $10.1017 / \mathrm{jfm} .2015 .21$.

[29] L.W. Dean. "Broadband noise generation by airfoils in turbulent flow". In: 4th Fluid and Plasma Dynamics Conference (1971). DOI: doi:10.2514/6.1971-587.

[30] B.D. Mugridge. "Acoustic radiation from aerofoils with turbulent boundary layers". In: Journal of Sound and Vibration 16.4 (June 1971), pp. 593-614. ISSN: 0022-460X. DOI: 10.1016/0022-460X (71)90665-1.

[31] Martin R Fink. "Investigation of Scrubbing and Impingement Noise". In: NASA Contractor Reports February 1975 (1975), p. 153.

[32] Martin V. Masek Fink. "Experimental Evaluation of Theories for Trailing Edge and Incidence Fluctuation Noise". In: AIAA Journal 13.11 (Nov. 1975), pp. 1472-1477. ISSN: 0001-1452. DOI: $\underline{10.2514 / 3.60559}$.

[33] T F Brooks, S Pope, and M A Marcolini. "Airfoil Self-Noise and Prediction". In: NASA Reference Publication 1218 (1989), pp. 1-142. ISSN: 14786095. DOI: 10.1080/09524622. 2008.9753825 .

[34] W Bechara and C Bailly. "Stochastic approach to noise modeling for free turbulent flows". In: AIAA Journal 32.3 (1994), pp. 455-463. ISSN: 0001-1452. DOI: 10.2514/3.12008.

[35] M. Gennaretti, L. Luceri, and L. Morino. "A UNIFIED BOUNDARY INTEGRAL METHODOLOGY FOR AERODYNAMICS AND AEROACOUSTICS OF ROTORS". In: Journal of Sound and Vibration 200.4 (Mar. 1997), pp. 467-489. ISSN: 0022-460X. DOI: $10.1006 /$ JSVI. 1996.0713.

[36] Gianfranco Guidati et al. "Simulation and measurement of inflow-turbulence noise on airfoils". In: 3rd AIAA/CEAS Aeroacoustics Conference. Reston, Virigina: American Institute of Aeronautics and Astronautics, May 1997. DOI: 10.2514/6.1997-1698.

[37] Christophe Bailly and Daniel Juvé. "A stochastic approach to compute subsonic noise using linearized Euler's equations". In: 5th AIAA/CEAS Aeroacoustic Conference and Exhibit c (1999), pp. 496-506. DOI: 10.2514/6.1999-1872.

[38] David P. Lockard and Philip J. Morris. "Radiated noise from airfoils in realistic mean flows". In: AIAA Journal 36.6 (June 2012), pp. 907-914. ISSN: 0001-1452. DOI: $\underline{10.2514 / 3.13912}$

[39] Sheryl M Grace. "Implications of recent bvi calculations on prediction methods for noise from turbulence ingestion". In: (2000). 
[40] J Casper and F Farassat. "Broadband Noise Predictions For an Airfoil in a Turbulent Stream ". In: 41st Aerospace Sciences Meeting and Exhibit (Jan. 2003), pp. 0-12. DOI: $10.2514 / 6.2003-366$.

[41] Patrick Moriarty. "Development and Validation of a Semi-Empirical Wind Turbine Aeroacoustic Code". In: 42nd AIAA Aerospace Sciences Meeting and Exhibit. Reston, Virigina: American Institute of Aeronautics and Astronautics, Jan. 2004. ISBN: 978-162410-078-9. DOI: $\underline{10.2514 / 6.2004-1189}$.

[42] Stephane Stéphane Moreau, Michel Roger, and Vincent Jurdic. "Effect of Angle of Attack and Airfoil Shape on Turbulence-Interaction Noise". In: AIAA Aeroacoustics Conference. May. Monterey, California: 11th AIAA/CEAS Aeroacoustics Conference Meeting and Exhibit, 2005, pp. 2005-2973. ISBN: 978-1-62410-052-9. DOI: 10.2514/6.2005-2973.

[43] Anurag Agarwal and Philip J. Morris. "Prediction Method for Broadband Noise from Unsteady Flow in a Slat Cove". In: AIAA Journal 44.2 (2006), pp. 301-310. ISSN: 0001-1452. DOI: 10.2514/1.12991.

[44] Massimo Gennaretti and Giovanni Bernardini. "Novel Boundary Integral Formulation for Blade-Vortex Interaction Aerodynamics of Helicopter Rotors". In: AIAA Journal 45.6 (June 2007), pp. 1169-1176. ISSN: 0001-1452. DOI: 10.2514/1.18383.

[45] Qidou Zhou and Phillip Joseph. "A frequency domain numerical method for airfoil broadband self-noise prediction". In: Journal of Sound and Vibration 299.3 (Jan. 2007), pp. 504-519. ISSN: 0022-460X. DOI: 10.1016/J.JSV.2006.06.061.

[46] Roland Ewert. "Broadband slat noise prediction based on CAA and stochastic sound sources from a fast random particle-mesh (RPM) method". In: Computers and Fluids 37.4 (2008), pp. 369-387. ISSN: 00457930. DOI: 10.1016/j.compfluid.2007.02.003.

[47] D. Lockard and M. Choudhari. "Noise Radiation from a Leading-Edge Slat". In: 15th AIAA/CEAS Aeroacoustics Conference (30th AIAA Aeroacoustics Conference) May (2009), pp. 11-13. DOI: $10.2514 / 6.2009-3101$.

[48] William J. Devenport, Joshua K. Staubs, and Stewart A.L. Glegg. "Sound radiation from real airfoils in turbulence". In: Journal of Sound and Vibration 329.17 (2010), pp. 3470-3483. ISSN: 0022460X. DOI: 10.1016/j.jsv.2010.02.022.

[49] Stewart A.L. L Glegg and William J. Devenport. "Panel methods for airfoils in turbulent flow". In: Journal of Sound and Vibration 329.18 (2010), pp. 3709-3720. ISSN: 0022460X. DOI: $10.1016 / j \cdot j s v .2010 .03 .007$.

[50] Damiano Casalino and Mattia Barbarino. "A Stochastic Method for Airfoil Self-Noise Computation in Frequency-Domain". In: AIAA Journal 49.11 (2010), p. 3884. ISSN: 0001-1452. DOI: $\underline{10.2514 / 1 . ~ J 050773}$.

[51] Florence V. Hutcheson, Thomas F. Brooks, and Daniel J. Stead. "Measurement of the Noise Resulting from the Interaction of Turbulence with a Lifting Surface". In: International Journal of Aeroacoustics 11.5-6 (2012), pp. 675-700. ISSN: 1475-472X.

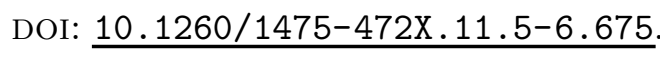

[52] T F Geyer, E Sarradj, and M Hobracht. "Noise generated by a leading edge in anisotropic turbulence". In: Inter-Noise. 1. Hamburg, 2016, pp. 6067-6078. 
[53] M. Dieste and G. Gabard. "Random particle methods applied to broadband fan interaction noise". In: Journal of Computational Physics 231.24 (Oct. 2012), pp. 81338151. URL: $10.1016 / \mathrm{J}$. JCP. 2012.07.044.

[54] Jae Wook Kim and Sina Haeri. "An advanced synthetic eddy method for the computation of aerofoil-turbulence interaction noise". In: Journal of Computational Physics 287 (2015), pp. 1-17. ISSN: 00219991. DOI: 10.1016/j.jcp.2015.01.039.

[55] Renato Fuzaro Miotto et al. "NUMERICAL COMPUTATION OF AIRFOIL-GUST COMPRESSIBLE LIFT RESPONSE”. In: (2017). DOI: 10. 26678/ABCM. COBEM2017. COB17-0253.

[56] Fernando Gea-Aguilera, James Gill, and Xin Zhang. "Synthetic turbulence methods for computational aeroacoustic simulations of leading edge noise". In: Computers and Fluids 157 (2017), pp. 240-252. ISSN: 00457930. DOI: 10.1016/j.compfluid.2017.08.039.

[57] M. Klein, A. Sadiki, and J. Janicka. "A digital filter based generation of inflow data for spatially developing direct numerical or large eddy simulations". In: Journal of Computational Physics 186.2 (Apr. 2003), pp. 652-665. ISSN: 00219991. DOI: 10.1016/ S0021-9991(03)00090-1.

[58] Robert H. Kraichnan. "Diffusion by a Random Velocity Field". In: Physics of Fluids 13.1 (1970), p. 22. URL: 10.1063/1.1692799.

[59] A. Careta, F. Gagues, and J. M. Sanch. "Stochastic generation of homogeneous isotropic turbulence with well-defined spectra". In: Physical Review E 48.3 (1993), pp. 2279-2287.

[60] P. E. Doak. "Acoustic radiation from a turbulent fluid containing foreign bodies". In: Proceedings of the Royal Society A: Mathematical, Physical and Engineering Sciences 254.1276 (1960).

[61] I. J. Sharland. "Sources of Noise in Axial Flow Fans". In: Journal of Sound and Vibration 1.3 (1964), pp. 302-322.

[62] R Amiet and W. R. Sears. "The aerodynamic noise of small-perturbation subsonic flows". In: Journal of Fluid Mechanics 44.02 (Nov. 1970), pp. 227-235.

[63] Peter D. Lysakn, Dean E. Capone, and Michael L. Jonson. "Prediction of high frequency gust response with airfoil thickness effects". In: Journal of Fluids and Structures 39 (2013), pp. 258-274.

[64] Massimo Gennaretti and Giovanni Bernardini. "Novel Boundary Integral Formulation for Blade-Vortex Interaction Aerodynamics of Helicopter Rotors". In: AIAA Journal 45.6 (June 2007), pp. 1169-1176. URL: 10.2514/1.18383.

[65] Donald Rockwell. "VORTEX-BODY INTERACTIONS". In: Annual Review of Fluid Mechanics 30.1 (Jan. 1998), pp. 199-229. URL: 10.1146/annurev.fluid.30.1.199.

[66] M. Gennaretti, L. Luceri, and L. Morino. "A unified boundary integral methodology for aerodynamics and aeroacoustics of rotors". In: Journal of Sound and Vibration 200.4 (Mar. 1997), pp. 467-489. URL: 10.1006/JSVI.1996.0713. 
[67] Stewart A.L. L Glegg and William J. Devenport. "Panel methods for airfoils in turbulent flow". In: Journal of Sound and Vibration 329.18 (2010), pp. 3709-3720. URL: 10.1016/ j.jsv.2010.03.007.

[68] Sheryl M Grace. "Unsteady blade response: The BVI model vs. The gust model". In: 7th AIAA/CEAS Aeroacoustics Conference May 28-30, 2001/Maastricht, The Netherlands For (2001).

[69] Mathias Basner et al. Auditory and non-auditory effects of noise on health. Apr. 2014. DOI: $10.1016 / \mathrm{S} 0140-6736(13) 61613-\mathrm{X}$.

[70] D.L. Sutliff et al. "Low-Speed Fan Noise Reduction with Trailing EDGE Blowing". In: International Journal of Aeroacoustics 1.3 (Sept. 2002), pp. 275-305. ISSN: 1475-472X. DOI: $10.1260 / 147547202320962592$.

[71] Daniel Crichton, Liping Xu, and Cesare A. Hall. "Preliminary Fan Design for a Silent Aircraft". In: Journal of Turbomachinery 129.1 (Jan. 2007), p. 184. ISSN: 0889504X. DOI: $\underline{10.1115 / 1.2372779}$.

[72] Sparsh Sharma and Ennes Sarradj. "Fluctuating Inflow Condition for Time-Domain Boundary Element Method for Airfoil-Turbulence Interaction Noise". In: ISUAAAT15 2018. Oxford, UK, 2018.

[73] Franke E. Fish and Juliann M. Battle. In: Journal of Morphology 225.1 (1995), pp. 51-60.

[74] V. Clair et al. "Experimental and Numerical Investigation of Turbulence-Airfoil Noise Reduction Using Wavy Edges". In: AIAA Journal 51.11 (Nov. 2013), pp. 2695-2713. ISSN: 0001-1452. DOI: 10.2514/1. J052394.

[75] Thomas F Geyer et al. "Silent owl flight: The effect of the leading edge comb". In: International Journal of Aeroacoustics 16.3 (Apr. 2017), pp. 115-134. ISSN: 1475-472X. DOI: $10.1177 / 1475472 \times 17706131$.

[76] B. C. Basu and G. J. Hancock. "The unsteady motion of a two dimensional aerofoil in incompressible inviscid flow". In: Journal of Fluid Mechanics 87.1 (1978), pp. 159-178.

[77] A Leonard. Vortex methods for flow simulation. Oct. 1980. DOI: 10.1016/00219991(80)90040-6.

[78] J Thomas Beale and Andrew Majda. Vortex Methods. I: Convergence in Three Dimensions. Tech. rep. 1982, pp. 1-27.

[79] P Ploumhans and G. S. Winckelmans. "Vortex Methods for High-Resolution Simulations of Viscous Flow Past Bluff Bodies of General Geometry". In: Journal of Computational Physics 165.2 (Dec. 2000), pp. 354-406. ISSN: 00219991. DOI: 10.1006/jcph.2000.6614.

[80] John Moores. "Potential Flow 2-Dimensional Vortex Panel Model: Applications to Wingmills". PhD thesis. 2003.

[81] Grégoire Winckelmans et al. Vortex methods and their application to trailing wake vortex simulations. May 2005. DOI: 10.1016/j.crhy.2005.05.001.

[82] Leandro D Santana, Christophe Schram, and Wim Desmet. "Panel method for turbulence-airfoil interaction noise prediction". In: 18th AIAA/CEAS Aeroacoustics Conference. 2012, pp. 1-10. 
[83] Georges-Henri Cottet and Petros D. Koumoutsakos. Vortex methods : theory and practice. Cambridge University Press, 2000, p. 313.

[84] J.L. L. Hess and A.M.O. M. O Smith. "Calculation of potential flow about arbitrary bodies". In: Progress in Aeronautical Sciences 8.1 (Jan. 1967), pp. 1-138. ISSN: 03760421. DOI: $10.1016 / 0376-0421$ (67)90003-6. URL: https : //www . sciencedirect. com/science/article/pii/0376042167900036.

[85] Joseph Katz and Allen Plotkin. Low Speed Aerodynamics. February 2013. Cambridge: Cambridge University Press, 2001, p. 351. URL: 10.1017/CB09780511810329.

[86] Massimo. Cencini, Fabio. Cecconi, and Angelo Vulpiani. Chaos: From simple models to Complex Systems. Vol. 17. World Scientific, 2010, p. 460. ISBN: 978-981-4277-65-5. DOI: 10.1142/7351. arXiv: arXiv:1011.1669v3.

[87] Ngai-Huat Teng. "The development of a computer code (U2DIIF)-for the numerical solution of unsteady". In: (1987).

[88] A. Schwarzenberg-Czerny. "On matrix factorization and efficient least squares solution." In: Astronomy and Astrophysics Supplement Series 110 (Apr. 1995), p. 405. ISSN: 0365-0138.

[89] Travis Oliphant. NumPy: A guide to NumPy. USA: Trelgol Publishing. 2006-. URL: http: //www. numpy.org/.

[90] Joseph P. Giesing. "Nonlinear two-dimensional unsteady potential flow with lift". In: Journal of Aircraft 5.2 (Mar. 1968), pp. 135-143. ISSN: 00218669. DOI: 10.2514/3.43920.

[91] Tuncer Cebeci et al. Analysis of Low-Speed Unsteady Airfoil Flows. Berlin, Heidelberg: Springer Berlin Heidelberg, 2005. ISBN: 3540229329. DOI: 10.1007/b138967.

[92] Arnold M Kuethe and Chuen-Yen Chow. Foundations of aerodynamics : bases of aerodynamic design. J. Wiley, 1998. ISBN: 0471129194.

[93] Lionel Schouveiler, F. S. Hover, and M. S. Triantafyllou. "Performance of flapping foil propulsion". In: Journal of Fluids and Structures 20.7 SPEC. ISS. (2005), pp. 949-959. ISSN: 10958622. DOI: $10.1016 / \mathrm{j}$.jfluidstructs.2005.05.009.

[94] X. Xia and K. Mohseni. "Unsteady aerodynamics and vortex-sheet formation of a two-dimensional airfoil". In: Journal of Fluid Mechanics 830 (Nov. 2017), pp. 439-478. ISSN: 0022-1120. DOI: $10.1017 / \mathrm{jfm} .2017 .513$. arXiv: 1611.05729.

[95] K W Moored. "Unsteady Three-Dimensional Boundary Element Method for SelfPropelled Bio-Inspired Locomotion”. In: Computers and Fluids (2017).

[96] Khaled Ibrahim Tolba and Guido Morgenthal. "Modelling of inflow-conditions for vortex particle methods to simulate atmospheric turbulence and its induced aerodynamic admittance on line-like bluff bodies". In: International Journal of Computational Fluid Dynamics 32.10 (2018), pp. 412-443. ISSN: 10290257. DOI: 10.1080/10618562.2018. 1542132.

[97] Rio Yokota and Shinnosuke Obi. "Vortex Methods for the Simulation of Turbulent Flows: Review". In: Journal of Fluid Science and Technology 6 (Jan. 2011), pp. 14-29. DOI: $10.1299 / \mathrm{jfst} .6 .14$. 
[98] M. L. Ould-Salihi, G. H. Cottet, and M. El Hamraoui. "Blending Finite-Difference and Vortex Methods for Incompressible Flow Computations". In: SIAM Journal on Scientific Computing 22.5 (Jan. 2001), pp. 1655-1674. ISSN: 1064-8275. DOI: $10.1137 /$ $\underline{\mathrm{S} 1064827599350769}$.

[99] Tsuyoshi Hamada et al. " 42 TFlops hierarchical $<\mathrm{i}>\mathrm{N}</ \mathrm{i}>$-body simulations on GPUs with applications in both astrophysics and turbulence". In: Proceedings of the Conference on High Performance Computing Networking, Storage and Analysis - SC '09. New York, New York, USA: ACM Press, 2009, p. 1. ISBN: 9781605587448. DOI: $10.1145 / 1654059.1654123$.

[100] By L Rosenhead and St John. "The Formation of Vortices from a Surface of Discontinuity". In: Proceedings of the Royal Society A: Mathematical, Physical and Engineering Sciences 134.823 (1931).

[101] Alexandre Joel Chorin and Peter S Bernard. "Discretization of a vortex sheet, with an example of roll-up". In: Journal of Computational Physics 13.3 (Nov. 1973), pp. 423-429. ISSN: 0021-9991. DOI: 10.1016/0021-9991(73)90045-4.

[102] Ole Hald, Vincenza Mauceri, and Del Prête. Convergence of Vortex Methods for Euler's Equations. Tech. rep. 143. 1978, pp. 791-809.

[103] L Greengard and V Rokhlin. "A fast algorithm for particle simulations". In: Journal of Computational Physics 73.2 (Dec. 1987), pp. 325-348. ISSN: 00219991. DOI: 10.1016/ 0021-9991(87)90140-9.

[104] Josh Barnes and Piet Hut. "A hierarchical O(N log N) force-calculation algorithm". In: Nature 324.6096 (Dec. 1986), pp. 446-449. ISSN: 0028-0836. DOI: 10.1038/324446a0.

[105] Adrian Sescu and Ray Hixon. "Toward low-noise synthetic turbulent inflow conditions for aeroacoustic calculations". In: International Journal for Numerical Methods in Fluids 73 (2013), pp. 1001-1010. ISSN: 02712091. DOI: 10.1002/fld. arXiv: fld.1 [DOI: 10.1002$]$.

[106] Rixin Yu and Xue-Song Song Bai. "A fully divergence-free method for generation of inhomogeneous and anisotropic turbulence with large spatial variation". In: Journal of Computational Physics 256 (Jan. 2014), pp. 234-253.

[107] Daehwan Kim, Seung Heo, and Cheolung Cheong. "Time-domain inflow boundary condition for turbulence-airfoil interaction noise prediction using synthetic turbulence modeling". In: Journal of Sound and Vibration 340 (Mar. 2015), pp. 138-151. ISSN: 0022-460X. DOI: 10.1016/J. JSV.2014.11.036.

[108] Baolin Xie et al. "Improved vortex method for large-eddy simulation inflow generation". In: Computers and Fluids 168.May (2018), pp. 87-100. ISSN: 00457930. DOI: 10.1016/ j.compfluid.2018.03.069.

[109] Luiz Antonio Alcântara Pereira, Miguel Hiroo Hirata, and Nelson Manzanares Filho. "Wake and aerodynamics loads in multiple bodies - application to turbomachinery blade rows". In: Journal of Wind Engineering and Industrial Aerodynamics 92.6 (May 2004), pp. 477-491. ISSN: 0167-6105. DOI: 10.1016/J. JWEIA. 2004.02.001. 
[110] Johannes Tophøj Rasmussen et al. "Discrete vortex method simulations of the aerodynamic admittance in bridge aerodynamics". English. In: Journal of Wind Engineering Industrial Aerodynamics 98.12 (2010), pp. 754-766. ISSN: 0167-6105. DOI: $10.1016 / j \cdot$ jweia.2010.06.011.

[111] Mads Mølholm Hejlesen et al. "On estimating the aerodynamic admittance of bridge sections by a mesh-free vortex method". In: Journal of Wind Engineering and Industrial Aerodynamics 146 (Nov. 2015), pp. 117-127. ISSN: 0167-6105. DOI: 10.1016/J. JWEIA. 2015.08.003.

[112] Sharma Sparsh and Sarradj Ennes. "Fluctuating inflow condition for time-domain boundary element method". In: Accepted for presentation at ISUAAAT15, University of Oxford, UK (2018).

[113] Peter S. Johansson and Helge I. Andersson. "Generation of inflow data for inhomogeneous turbulence". In: Theoretical and Computational Fluid Dynamics 18.5 (Nov. 2004), pp. 371-389. URL: $10.1007 / \mathrm{s} 00162-004-0147-\mathrm{z}$.

[114] P Druault et al. "Generation of Three-Dimensional Turbulent Inlet Conditions for Large-Eddy Simulation". In: AIAA JOURNAL 42.3 (2004). URL: 10.2514/1.3946.

[115] David M. F. Chapman. "Ideal vortex motion in two dimensions: Symmetries and conservation laws". In: Journal of Mathematical Physics 19.9 (1978), p. 1988. URL: $10.1063 / 1.523897$.

[116] R. Benzi, S. Succi, and M. Vergassola. "The lattice Boltzmann equation: theory and applications". In: Physics Reports 222.3 (Dec. 1992), pp. 145-197. URL: 10.1016/0370$1573(92) 90090-\mathrm{M}$.

[117] Dalila Elhma‘idi, Antonello Provenzale, and Armando Babiano. "Elementary topology of two-dimensional turbulence from a Lagrangian viewpoint and single-particle dispersion". In: Journal of Fluid Mechanics 257.-1 (Dec. 1993), p. 533. URL: 10.1017/ S0022112093003192.

[118] J. G. Esler and T. L. Ashbee. "Universal statistics of point vortex turbulence". In: Journal of Fluid Mechanics 779 (Sept. 2015), pp. 275-308. URL: 10.1017/jfm.2015.410.

[119] T von Kármán. "Turbulence: Twebty-fifth Wilbur Wright Memorial Lecture". In: Journal of Royal Aeronautical Society (1937), p. 1109.

[120] J. O. Hinze. Turbulence. McGraw-Hill, 1975, p. 790. ISBN: 0070290377.

[121] Javier Jiménez. Turbulence and vortex dynamics. Tech. rep. 2004.

[122] Christophe Bailly and Geneviève Comte-Bellot. Turbulence. Experimental Fluid Mechanics. Cham: Springer International Publishing, 2015. ISBN: 978-3-319-16159-4. DOI: $10.1007 / 978-3-319-16160-0$.

[123] Tuncer. Cebeci and Tuncer. Cebeci. Analysis of turbulent flows. Elsevier, 2004, p. 376. ISBN: 9780080443508.

[124] Jean Piquet. Turbulent Flows. Berlin, Heidelberg: Springer Berlin Heidelberg, 1999. ISBN: 9783642084751. DOI: $10.1007 / 978-3-662-03559-7$. 
[125] Lars Davidson. Fluid mechanics, turbulent flow and turbulence modeling. Tech. rep. Sweden: Chalmers University of Technology, 2019.

[126] A. N. Kolmogorov. The Local Structure of Turbulence in Incompressible Viscous Fluid for Very Large Reynolds Numbers. DOI: 10.2307/51980.

[127] G. I. Taylor. Statistical Theory of Turbulence. DOI: 10.2307/96557.

[128] A. S. Monin, A. M. YaGlom, and John L. Lumley. Statistical fluid mechanics : mechanics of turbulence (Vol:2). Dover Publications, 2007. ISBN: 0486458911.

[129] Laurent. Schwartz and Denise Huet. Méthodes mathématiques pour les sciences physiques. Hermann, 1965. ISBN: 2705652132.

[130] Stephen B. Pope. Turbulent Flows. Cambridge University Press, 2000. DOI: $\underline{10.1017 /}$ CB09780511840531.

[131] T von Kármán. "Progress in the Statistical Theory of Turbulence." In: Proceedings of the National Academy of Sciences of the United States of America 34.11 (Nov. 1948), pp. 530-9. ISSN: 0027-8424. DOI: 10.1073/pnas.34.11.530.

[132] J. Gordon Leishman, Mahendra J. Bhagwat, and Ashish Bagai. "Free-Vortex Filament Methods for the Analysis of Helicopter Rotor Wakes". In: Journal of Aircraft 39.5 (Sept. 2008), pp. 759-775. ISSN: 0021-8669. DOI: 10.2514/2.3022.

[133] Hamidreza Abedi. "Development of vortex filament method for wind power aerodynamics". PhD thesis. 2016. ISBN: 9789175973609.

[134] William John Macquorn Rankine. A manual of applied mechanics. London: Griffen Co., 1858.

[135] M P. Scully et al. "Helicopter Rotor Wake Geometry and Airloads and Development of Laser Doppler Velocimeter for Use in Helicopter Rotor Wakes". In: (1972).

[136] G. H. Vatistas. "A simpler model for concentrated vortices". In: Experiments in Fluids 11.1 (Apr. 1991), pp. 73-76. ISSN: 0723-4864. DOI: 10.1007/BF00198434.

[137] Ashish Bagai and J. Gordon Leishman. "Flow visualization of compressible vortex structures using density gradient techniques". In: Experiments in Fluids 15.6 (Oct. 1993), pp. 431-442. ISSN: 07234864. DOI: $10.1007 / \mathrm{BF} 00191786$.

[138] Ole H. Hald. "Convergence of Vortex Methods for Euler's Equations. II". In: SIAM Journal on Numerical Analysis 16.5 (Oct. 1979), pp. 726-755. ISSN: 0036-1429. DOI: 10.1137/0716055.

[139] J. Thomas Beale. "On the Accuracy of Vortex Methods at Large Times". In: Springer, New York, NY, 1988, pp. 19-32. DOI: 10.1007/978-1-4612-3882-9_2.

[140] Jeff D. Eldredge, Tim Colonius, and Anthony Leonard. "A vortex particle method for two-dimensional compressible flow". In: Journal of Computational Physics 179.2 (July 2002), pp. 371-399. ISSN: 00219991. DOI: 10.1006/jcph.2002.7060.

[141] N. Jarrin et al. "A synthetic-eddy-method for generating inflow conditions for large-eddy simulations". In: International Journal of Heat and Fluid Flow 27.4 (2006), pp. 585-593. URL: $10.1016 / j$. ijheatfluidflow.2006.02.006. 
[142] Adrian Sescu and Ray Hixon. "Toward low-noise synthetic turbulent inflow conditions for aeroacoustic calculations". In: International Journal for Numerical Methods in Fluids 73 (2013), pp. 1001-1010. URL: 10.1002/fld.

[143] Marie Farge. "Wavelet Transforms And Their Applications To Turbulence". In: Annual Review of Fluid Mechanics 24.1 (1992), pp. 395-457. ISSN: 00664189. DOI: 10.1146/ annurev.fluid.24.1.395.

[144] M. S. Howe. "On sound generated when a vortex is chopped by a circular airfoil". In: Journal of Sound and Vibration 128.3 (Feb. 1989), pp. 487-503. ISSN: 10958568. DOI: 10.1016/0022-460X (89)90787-6.

[145] Julius S. Bendat and Allan G. Piersol. Random Data: Analysis and Measurement Procedures: Fourth Edition. 2012, pp. 1-613. ISBN: 9781118032428. DOI: $10.1002 /$ 9781118032428.

[146] Choosing an appropriate distribution - Analytica Wiki. Tech. rep. Analytica, 2019, pp. $1-4$.

[147] T Yehoshua and A Seifert. "Empirical Model for the Evolution of a Vortex-Pair Introduced into a Boundary Layer". In: AerospaceLab 6 (2013), p. 1-12.

[148] Norbert Didden. "On the formation of vortex rings: Rolling-up and production of circulation". In: Zeitschrift für angewandte Mathematik und Physik ZAMP 30.1 (Jan. 1979), pp. 101-116. ISSN: 0044-2275. DOI: 10.1007/BF01597484.

[149] Bruce J Wendt. Initial Circulation and Peak Vorticity Behavior of Vortices Shed From Airfoil Vortex Generators. Tech. rep. 2001.

[150] T. Yehoshua and A. Seifert. "Boundary condition effects on the evolution of a train of vortex pairs in still air". In: The Aeronautical Journal 110.1109 (July 2006), pp. 397-417. ISSN: 0001-9240. DOI: 10.1017/S0001924000001317.

[151] A Favrel et al. "Space and time reconstruction of the precessing vortex core in Francis turbine draft tube by 2D-PIV". In: IOP Conference Series: Earth and Environmental Science 49 (Nov. 2016), p. 082011. ISSN: 1755-1307. DOI: 10.1088/1755-1315/49/8/ $\underline{082011 .}$.

[152] Mark Peter Rast and Jean Francois Pinton. "Point-vortex model for Lagrangian intermittency in turbulence". In: Physical Review E - Statistical, Nonlinear, and Soft Matter Physics 79.4 (2009), pp. 1-12. ISSN: 15393755. DOI: 10.1103/PhysRevE.79. 046314.

[153] Kenneth Levenberg. "A method for the solution of certain non-linear problems in least squares". In: Quarterly of Applied Mathematics 2.2 (July 1944), pp. 164-168. ISSN: 0033-569X. DOI: $10.1090 /$ qam/10666.

[154] Donald W. Marquardt. "An Algorithm for Least-Squares Estimation of Nonlinear Parameters". In: Journal of the Society for Industrial and Applied Mathematics 11.2 (June 1963), pp. 431-441. DOI: 10.1137/0111030.

[155] Eric Jones, Travis Oliphant, Pearu Peterson, et al. SciPy: Open source scientific tools for Python. 2001-. URL: http://www.scipy.org/. 
[156] Alan V. Oppenheim and George C. Verghese. Signals, Systems and Inference, Global Edition. 1/E. Pearson, 2016. ISBN: 9781292156200.

[157] Claus Wagner, Thomas Hüttl, and Pierre Sagaut. Large-eddy simulation for acoustics. Ed. by Claus Wagner, Thomas Hüttl, and Pierre Sagaut. Vol. 9780521871. Cambridge: Cambridge University Press, 2007, pp. 1-441. ISBN: 9780511546143. DOI: $10.1017 /$ CB09780511546143.

[158] L Gutin. On the sound field of a rotating propeller. Tech. rep. NASA, Oct. 1948.

[159] Alan Powell. "Aerodynamic noise and the plane boundary". In: Journal of the Acoustical Society of America 32.8 (Aug. 1960), pp. 982-990. ISSN: NA. DOI: 10.1121/1.1908347.

[160] J. E. Ffowcs Williams and D. L. Hawkings. "Sound Generation by Turbulence and Surfaces in Arbitrary Motion". In: Philosophical Transactions of the Royal Society of London A: Mathematical, Physical and Engineering Sciences 264.1151 (1969).

[161] Avraham Hirschberg. "Introduction to Aeroacoustics and Self-Sustained Oscillations of Internal Flows". In: CISM International Centre for Mechanical Sciences, Courses and Lectures. Vol. 545. Springer, Vienna, 2013, pp. 3-72. DOI: 10.1007/978-3-7091-1458$\underline{2} 1$.

[162] Y Auregan et al. Sound-flow interactions. Ed. by Yves Aurégan et al. Vol. 586. Lecture Notes in Physics. Berlin, Heidelberg: Springer Berlin Heidelberg, 2011, p. 286. ISBN: 3540433325. DOI: $10.1007 / 3-540-45880-8$.

[163] Stewart Glegg and William Devenport. Aeroacoustics of Low Mach Number Flows. 1st ed. Academic Press, 2017, p. 529. ISBN: 9780128096512.

[164] Vasanth Allampalli et al. "High-accuracy large-step explicit Runge-Kutta (HALE-RK) schemes for computational aeroacoustics". In: Journal of Computational Physics 228.10 (June 2009), pp. 3837-3850. ISSN: 00219991. DOI: 10.1016/j.jcp.2009.02.015.

[165] Stewart A.L. Glegg and William Devenport. "Unsteady loading on an airfoil of arbitrary thickness". In: Journal of Sound and Vibration 319.3-5 (Jan. 2009), pp. 1252-1270. ISSN: 0022-460X. DOI: $10.1016 / \mathrm{J}$. JSV.2008.06.053.

[166] R.K. Amiet. "Noise due to turbulent flow past a trailing edge". In: Journal of Sound and Vibration 47.3 (Aug. 1976), pp. 387-393. ISSN: 0022460X. DOI: $10.1016 / 0022-$ 460X (76)90948-2.

[167] Roland Ewert et al. "RANS/CAA Based Prediction of NACA 0012 Broadband Trailing Edge Noise and Experimental Validation". In: 15th AIAA/CEAS Aeroacoustics Conference (30th AIAA Aeroacoustics Conference). Reston, Virigina: American Institute of Aeronautics and Astronautics, May 2009. IsBN: 978-1-60086-967-9. DOI: 10.2514/6. 2009-3269.

[168] Chaitanya Paruchuri et al. "Aerofoil geometry effects on turbulence interaction noise". In: 21st AIAA/CEAS Aeroacoustics Conference. Reston, Virginia: American Institute of Aeronautics and Astronautics, June 2015. ISBN: 978-1-62410-367-4. DOI: 10.2514/6. $\underline{2015-2830 .}$ 
[169] A M Hall et al. "Effect of Leading-Edge Thickness on High-Speed Airfoil-Turbulence Interaction Noise". In: AIAA Conference June (2011), pp. 05-08. DOI: 10.2514/6.2011$\underline{2861}$.

[170] Auris Juknevicius and Tze Pei Chong. "On the leading edge noise and aerodynamics of thin aerofoil subjected to the straight and curved serrations". In: Journal of Sound and Vibration 425 (2018), pp. 324-343. ISSN: 0022-460X. DOI: https://doi.org/10.1016/ j.jsv.2018.02.038. 



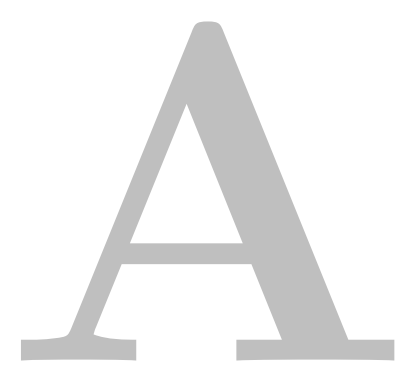

Appendix A

\section{A.1 Green's Functions and Solutions of Laplace's Equation}

The idea of Green's functions to solve the Laplace's and Poisson's equation in unbounded domains. A concise derivation of Green's identities that enable us to construct Green's functions for Laplace's equation and its inhomogeneous cousin, Poisson's equation.

\section{A.1.1 Poisson's equation}

Poisson's equation for a function $\phi: \mathbb{R}^{n} \rightarrow \mathbb{C}$ is the forced Laplace equation

$$
\nabla^{2} \phi=-F(\mathbf{x}),
$$

where as always the forcing term $F: \mathbb{R}^{n} \rightarrow \mathbb{C}$ is assumed to be given, and the minus sign on the rhs of Eq. (A.1) is conventional. We will again solve this equation by first constructing a Green's function for it, although the solution via Green's functions is less straightforward than for either the heat or wave equations - in particular, we will not obtain it via Fourier transforms (the Green's function itself will turn out not to be absolutely integrable). We also note that, since there is no time variable (the equation is elliptic), the solutions will have no notion of causality.

\section{A.1.1.1 The fundamental solution}

The fundamental solution $G_{n}$ to Poisson's equation in n-dimensions is defined to be the solution to the problem

$$
\nabla^{2} G_{n}(\mathbf{x} ; \mathbf{y})=\delta^{(n)}(\mathbf{x}-\mathbf{y}),
$$


where the forcing term is chosen to be just an n-dimensional $\delta$-function. Since the problem rotationally symmetric about the special point $\mathbf{y}$, the fundamental solution can only depend on the scalar distance from that point:

$$
G_{n}(\mathbf{x} ; \mathbf{y})=G_{n}(|\mathbf{x}-\mathbf{y}|) .
$$

Integrating both sides of Eq. $\underline{(\mathrm{A} .2)}$ over a ball

$$
B_{r}=\left\{\mathbf{x} \in \mathbb{R}^{n}:|\mathbf{x}-\mathbf{y}| \leq r\right\}
$$

of radius $r$ centred on $\mathbf{y}$ we obtain

$$
1=\int_{B_{r}} \nabla^{2} G_{n} \mathrm{~d} V=\int_{\partial B_{r}} \mathbf{n} \cdot \nabla G_{n} \mathrm{~d} S
$$

where the integral of the $\delta$-function gives 1 , and we have used the divergence theorem to reduce the integral of $\nabla^{2} G_{n}$ to an integral over the boundary sphere $\partial B_{r}=\left\{\mathbf{x} \in \mathbb{R}^{n}: \quad|\mathbf{x}-\mathbf{y}| \leq r\right\}$. When $n=3$, this is the surface of a 'standard' sphere, while it is a circle when $\mathrm{n}=2$ and a 'hypersphere' for $n>3$. In each case, the outward pointing normal $\mathrm{n}$ points in the radial direction, so $\mathbf{n} \cdot \nabla G_{n}=d G_{n} / d r$. Thus we obtain

$$
1=\int_{\partial B_{r}} \frac{d G_{n}}{d r} r^{n-1} \mathrm{~d} \Omega_{n}=r^{n-1} \frac{d G_{n}}{d r} \int_{S^{n-1}} \mathrm{~d} \Omega_{n},
$$

where we have used the fact that $G$ is a function only of the radius, and where $\mathrm{d} \Omega_{n}$ is the measure on a unit $S^{n-1}$; for example

$$
\mathrm{d} \Omega_{n}=\left\{\begin{array}{ll}
\sin \theta \mathrm{d} \theta \mathrm{d} \phi & \text { when } \mathrm{n}=3 \\
\mathrm{~d} \theta & \text { when } \mathrm{n}=2
\end{array} .\right.
$$

The remaining angular integral just gives the total 'surface area' $A_{n}$ of our $(n-1)^{\triangleleft}$ dimensional sphere. In particular, $A_{2}=2 \pi$ is the circumference of a circle, whereas $A_{3}=4 \pi$ is the total solid angle of a sphere in three dimensions. Then

$$
\frac{d G_{n}}{d r}=\frac{1}{A r^{n-1}}
$$

This ode is easily solved to show that the fundamental solution is

$$
G_{n}(\mathbf{x} ; \mathbf{y})= \begin{cases}|\mathbf{x}-\mathbf{y}|+c_{1} & n=1 \\ \frac{1}{2 \pi} \log |\mathbf{x}-\mathbf{y}|+c_{2} & n=2 . \\ -\frac{1}{A_{n}(n-2)} \frac{1}{|\mathbf{x}-\mathbf{y}|^{n-2}}+c_{n} & n \geq 3\end{cases}
$$

When $n \geq 3$ we can fix the constant $c_{n}$ to be zero by requiring $\lim _{r \rightarrow \infty} G(r)=0$; however, this condition cannot be imposed in either one or two dimensions, and some other condition (usually suggested by the details of a specific problem) must instead be imposed. Note that when $n>1$ these fundamental solutions satisfy the Laplace equation for all $\mathbf{x} \in \mathbb{R}^{n}-\{\mathbf{y}\}$, but that they become singular at $\mathbf{x}=\mathbf{y}$. 


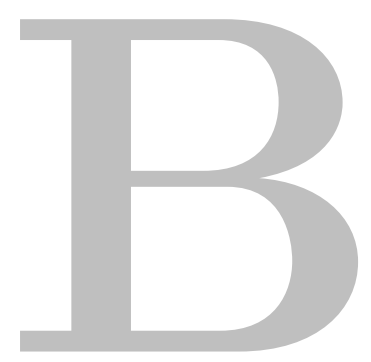

Appendix B

B.1 Spectral analysis of velocity signals of the vortex window of different lengths

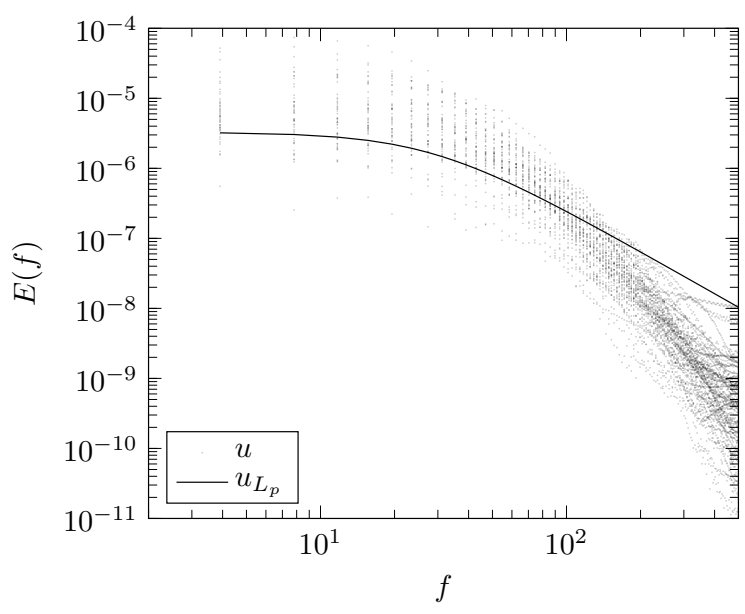

(a) longitudinal spectrum

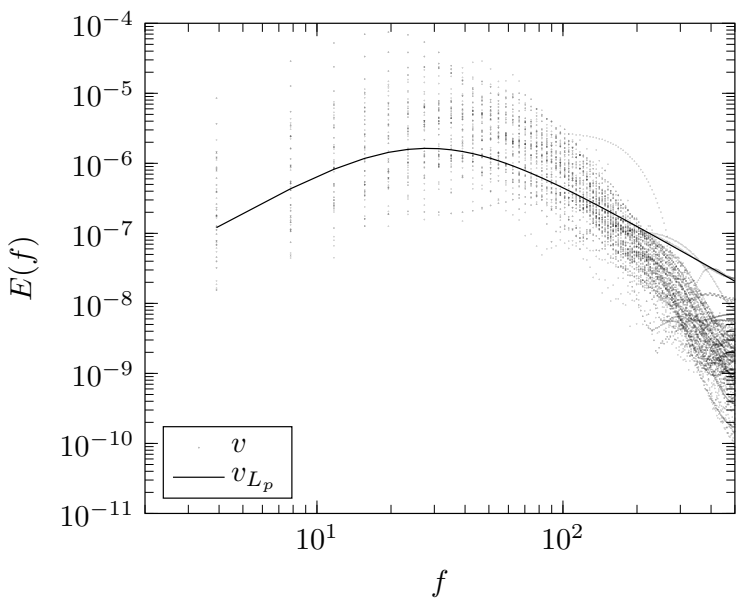

(b) transverse spectrum

Figure B.1: spectral analysis of velocity signals of the vortex window of 25 chord lengths long 0.2 chord length wide 


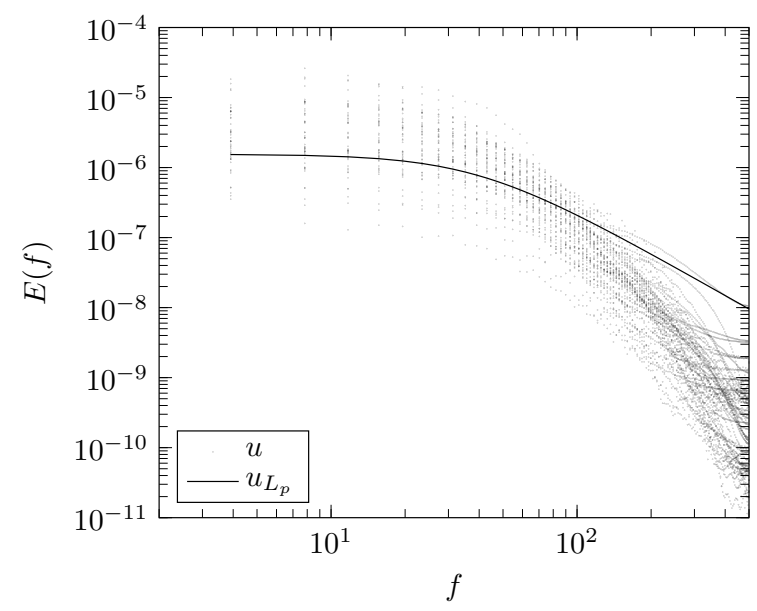

(a) 50-0.2-longitudinal spectrum

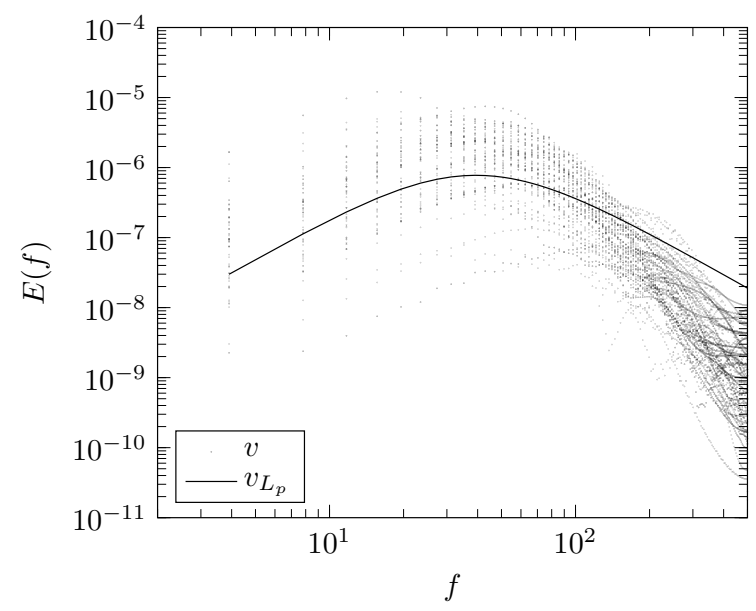

(b) 50-0.2-transverse spectrum

Figure B.2: spectral analysis of velocity signals of the vortex window of 50 chord lengths long 0.2 chord length wide

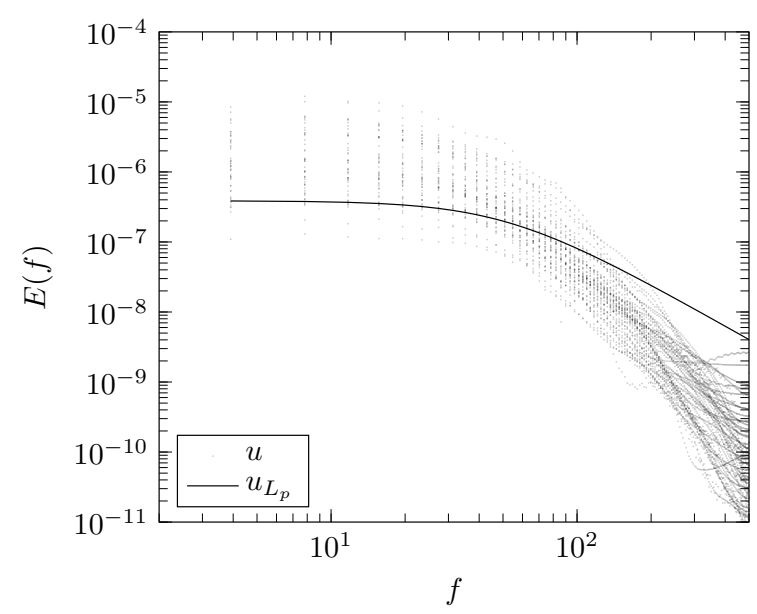

(a) 100-0.2-longitudinal spectrum

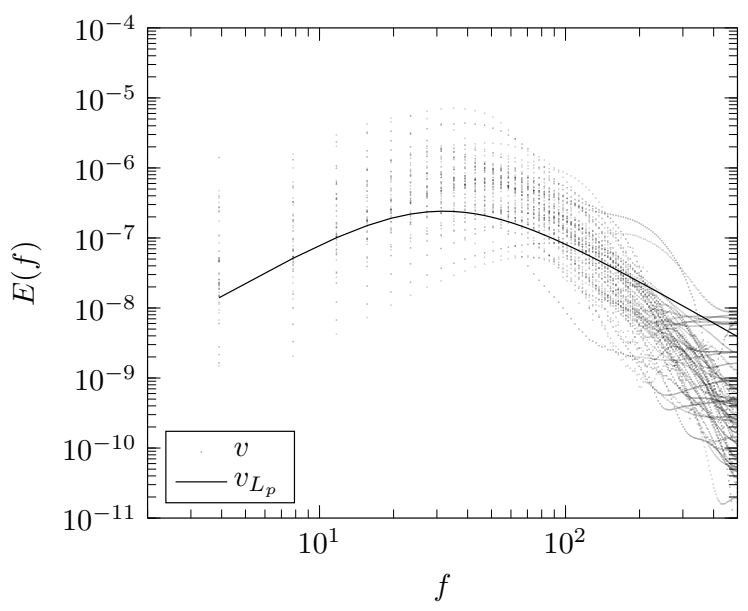

(b) 100-0.2-transverse spectrum

Figure B.3: spectral analysis of velocity signals of the vortex window of 100 chord lengths long 0.2 chord length wide 


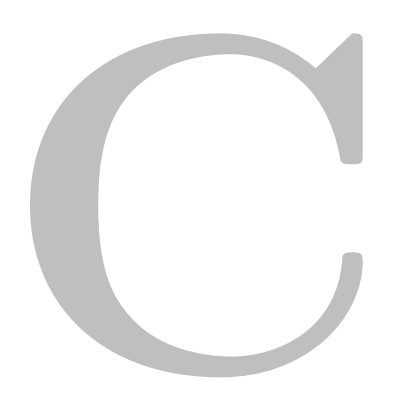

Appendix C

\section{C.1 Levenberg-Marquardt algorithm}

The Levenberg-Marquardt algorithm was developed in the early 1960's to solve nonlinear least squares problems. Least squares problems arise in the context of fitting a parameterised function to a set of measured data points by minimising the sum of the squares of the errors between the data points and the function. Nonlinear least squares methods iteratively reduce the sum of the squares of the errors between the function and the measured data points through a sequence of updates to parameter values. The Levenberg-Marquardt algorithm combines two minimisation methods: the gradient descent method and the Gauss-Newton method. In the gradient descent method, the sum of the squared errors is reduced by updating the parameters in the steepest-descent direction. In the Gauss-Newton method, the sum of the squared errors is reduced by assuming the least squares function is locally quadratic, and finding the minimum of the quadratic. The Levenberg-Marquardt method acts more like a gradient-descent method when the parameters are far from their optimal value, and acts more like the Gauss-Newton method when the parameters are close to their optimal value.

The primary application of the Levenberg-Marquardt algorithm is in the least-squares curve fitting problem: given a set of $m$ empirical datum pairs $\left(x_{i}, y_{i}\right)$ of independent and dependent variables, find the parameters $\beta$ of the model curve $f(x, \beta)$ so that the sum of the squares of the deviations $S(\beta)$ is minimised:

$$
\hat{\beta} \in \operatorname{argmin}_{\beta} S(\beta) \equiv \operatorname{argmin}_{\beta} \sum_{i=1}^{m}\left[y_{i}-f\left(x_{i}, \beta\right)\right]^{2},
$$

which is assumed to be non-empty.

\section{C.1.1 The solution}

Like other numeric minimisation algorithms, the Levenberg-Marquardt algorithm is an iterative procedure. To start a minimisation, the user has to provide an initial guess for the parameter vector $\beta$. In cases with only one minimum, an uninformed standard guess like 
$\beta^{\mathrm{T}}=(1,1, \ldots, 1)$ will work fine; in cases with multiple minima, the algorithm converges to the global minimum only if the initial guess is already somewhat close to the final solution. In each iteration step, the parameter vector $\beta$ is replaced by a new estimate $\beta+\delta$. To determine $\delta$, the function $f\left(x_{i}, \beta+\delta\right)$ is approximated by its linearisation:

$$
f\left(x_{i}, \beta+\delta\right) \approx f\left(x_{i}, \beta\right)+\mathbf{J}_{i} \delta
$$

where

$$
\mathbf{J}_{i}=\frac{\partial f\left(x_{i}, \beta\right)}{\partial \beta}
$$

is the gradient (row-vector in this case) of $f$ with respect to $\beta$.

The sum $S(\beta)$ of square deviations has its minimum at a zero gradient with respect to $\beta$. The above first-order approximation of $f\left(x_{i}, \beta+\delta\right)$ gives

$$
S(\beta+\delta) \approx \sum_{i=1}^{m}\left[y_{i}-f\left(x_{i}, \beta\right)-\mathbf{J}_{i} \delta\right]^{2}
$$

or in vector notation,

$$
\begin{aligned}
S(\beta+\delta) & \approx\|\mathbf{y}-\mathbf{f}(\beta)-\mathbf{J} \delta\|^{2} \\
& =[\mathbf{y}-\mathbf{f}(\beta)-\mathbf{J} \delta]^{\mathrm{T}}[\mathbf{y}-\mathbf{f}(\beta)-\mathbf{J} \delta] \\
& =[\mathbf{y}-\mathbf{f}(\beta)]^{\mathrm{T}}[\mathbf{y}-\mathbf{f}(\beta)]-[\mathbf{y}-\mathbf{f}(\beta)]^{\mathrm{T}} \mathbf{J} \delta-(\mathbf{J} \delta)^{\mathrm{T}}[\mathbf{y}-\mathbf{f}(\beta)]+\delta^{\mathrm{T}} \mathbf{J}^{\mathrm{T}} \mathbf{J} \delta \\
& =[\mathbf{y}-\mathbf{f}(\beta)]^{\mathrm{T}}[\mathbf{y}-\mathbf{f}(\beta)]-2[\mathbf{y}-\mathbf{f}(\beta)]^{\mathrm{T}} \mathbf{J} \delta+\delta^{\mathrm{T}} \mathbf{J}^{\mathrm{T}} \mathbf{J} \delta .
\end{aligned}
$$

Taking the derivative of $S(\beta+\delta)$ with respect to $\delta$ and setting the result to zero gives

$$
\left(\mathbf{J}^{\mathrm{T}} \mathbf{J}\right) \delta=\mathbf{J}^{\mathrm{T}}[\mathbf{y}-\mathbf{f}(\beta)],
$$

where $\mathbf{J}$ is the Jacobian matrix, whose $i$-th row equals $\mathbf{J}_{i}$, and where $\mathbf{f}(\beta)$ and $\mathbf{y}$ are vectors with $i$-th component $f\left(x_{i}, \beta\right)$ and $y_{i}$ respectively. This is a set of linear equations, which can be solved for $\delta$.

Levenberg's contribution is to replace this equation by a "damped version":

$$
\left(\mathbf{J}^{\mathrm{T}} \mathbf{J}+\lambda \mathbf{I}\right) \delta=\mathbf{J}^{\mathrm{T}}[\mathbf{y}-\mathbf{f}(\beta)],
$$

where $\mathbf{I}$ is the identity matrix, giving as the increment $\delta$ to the estimated parameter vector $\beta$. The (non-negative) damping factor $\lambda$ is adjusted at each iteration. If reduction of $S$ is rapid, a smaller value can be used, bringing the algorithm closer to the Gauss-Newton algorithm, whereas if an iteration gives insufficient reduction in the residual, $\lambda$ can be increased, giving a step closer to the gradient-descent direction. Note that the gradient of $S$ with respect to $\delta$ equals $-2\left(\mathbf{J}^{\mathrm{T}}[\mathbf{y}-\mathbf{f}(\beta)]\right)^{\mathrm{T}}$. Therefore, for large values of $\lambda$, the step will be taken approximately in the direction of the gradient. If either the length of the calculated step $\delta$ or the reduction of sum of squares from the latest parameter vector $\beta+\delta$ fall below predefined limits, iteration stops, and the last parameter vector $\beta$ is considered to be the solution. 
Levenberg's algorithm has the disadvantage that if the value of damping factor $\lambda$ is large, inverting $\mathbf{J}^{\mathrm{T}} \mathbf{J}+\lambda \mathbf{I}$ is not used at all. R. Fletcher provided the insight that we can scale each component of the gradient according to the curvature, so that there is larger movement along the directions where the gradient is smaller. This avoids slow convergence in the direction of small gradient. Therefore, Fletcher in his 1971 paper "A modified Marquardt subroutine for non-linear least squares" replaced the identity matrix $\mathbf{I}$ with the diagonal matrix consisting of the diagonal elements of $\mathbf{J}^{\mathrm{T}} \mathbf{J}$, thus making the solution scale invariant:

$$
\left[\mathbf{J}^{\mathrm{T}} \mathbf{J}+\lambda \operatorname{diag}\left(\mathbf{J}^{\mathrm{T}} \mathbf{J}\right)\right] \delta=\mathbf{J}^{\mathrm{T}}[\mathbf{y}-\mathbf{f}(\beta)]
$$

